Merci d'utiliser le titre suivant lorsque vous citez ce document :

Greene, M. et al. (2008-09-03), «Échanges et croissance de la Chine : Incidences sur certains pays de l'OCDE », Éditions OCDE, Paris.

http://dx.doi.org/10.1787/226877350450

\title{
Échanges et croissance de la Chine
}

\author{
INCIDENCES SUR CERTAINS PAYS DE L'OCDE
}

Malory Greene,

Nora Dihel,

Przemyslaw Kowalski,

Douglas C. Lippoldt

La version originale de ce document a été publiée comme suit :

Greene, M. et al. (2006-11-28), "China's Trade and Growth: Impact on Selected OECD Countries", OECD Trade Policy Papers, No. 44, OECD Publishing, Paris. http://dx.doi.org/10.1787/274600240481 
Organisation de Coopération et de Développement Économiques

Organisation for Economic Co-operation and Development

03-Sep-2008

DIRECTION DES ECHANGES

Français - Or. Anglais

COMITE DES ECHANGES

Groupe de travail du Comité des échanges

ÉCHANGES ET CROISSANCE DE LA CHINE : INCIDENCES SUR CERTAINS PAYS DE L'OCDE

Document de travail de l'OCDE sur la politique commerciale $n^{\circ} 44$

Par Malory Greene, Nora Dihel, Przemyslaw Kowalski et Douglas Lippoldt

Tous les Documents de travail sur la politique des échanges sont disponibles sur le site web de

l'OCDE, à l'adresse suivante : http://www/oecd.org/trade 


\section{RÉSUMÉ}

Le présent document examine l'émergence de la Chine en tant qu'acteur mondial sur les marchés internationaux au cours des toutes dernières décennies. Il offre une vue d'ensemble du cadre de la politique commerciale de la Chine après l'ouverture de ses marchés et son adhésion à l'OMC. Ce rapport analyse le rôle de la Chine dans les activités internationales de transformation et son élévation dans la chaîne de valeur mondiale. Il traite également de l'impact de la Chine sur les prix mondiaux et de la dégradation de ses termes de l'échange. Il étudie la stratégie poursuivie par la Chine, qui consiste à assurer l'expansion de ses exportations sur deux fronts. Le premier aspect de cette stratégie consiste à tirer parti du facteur de production qui constitue son atout majeur - sa main-d'œuvre surabondante - en privilégiant les produits manufacturés à forte intensité de main-d'œuvre, qui créent des emplois. Le second consiste à promouvoir son objectif de développement en modernisant son économie grâce à la production et à l'exportation de biens de haute technologie. Le document traite également des droits de propriété intellectuelle (DPI). La plupart des données font apparaître de considérables progrès dans la mise en place d'un régime de DPI adapté aux besoins d'une économie de marché moderne. Subsistent toutefois certaines déficiences - en particulier en ce qui concerne le contrôle de l'application de la réglementation en matière de DPI - qui pourraient porter préjudice aux intérêts nationaux et internationaux.

La dernière partie du rapport traite des répercussions de l'intégration de la Chine aux marchés mondiaux des biens et des services sur certains pays de l'OCDE. Pour quantifier l'impact de la Chine sur l'économie mondiale, elle a recours à de nouvelles estimations fondées sur un modèle d'équilibre général, baptisé "modèle FTAP". Sur la base des données de 2001, elle fournit des estimations chiffrées des effets sectoriels que l'intégration de la Chine dans les échanges mondiaux exerce sur les volumes de production comme sur les prix et en quantifie également l'impact sur le bien-être dans certaines économies de l'OCDE. Le rapport parvient à la conclusion que la mise en œuvre par la Chine des engagements pris à l'OMC ou une libéralisation totale dans le domaine des droits de douane et des services assurera des gains considérables à la Chine et aura un impact assez limité sur les économies de l’OCDE.

Mots-clés: Chine, pays de l'OCDE, politique commerciale, échanges et croissance, investissement, impact sur l'économie mondiale, droits de propriété intellectuelle (DPI), activités internationales de transformation, mobilité au sein de la chaîne de valeur, prix mondiaux, termes de l'échange, modèle d'équilibre général calculable, modèle FTAP, indice de restriction des échanges, commerce de services, banque, télécommunications et assurance.

\section{REMERCIEMENTS}

Le présent document a été rédigé en collaboration par Malory Greene, Nora Dihel, Przemek Kowalski et Douglas Lippoldt, tous trois de la Direction des échanges de l'OCDE. Il a été supervisé par Raed Safadi, Chef de la Division Dialogue sur les politiques commerciales, de la Direction des échanges de l'OCDE. D'autres personnes y ont également apporté leur contribution: Jane Korinek (Direction des échanges de l'OCDE) sur l'analyse statistique des chaînes de valeur, Philippa Dee (Université nationale australienne) sur le modèle FTAP et Raphael Espinoza (rattaché à l'Université d'Oxford) sur les travaux de modélisation. Ce document a bénéficié des précieux commentaires formulés par Margit Molnar et Sean Dougherty (tous deux membres du Département des affaires économiques de l'OCDE) et Ken Davies (Division de l'investissement de l'OCDE). Jacqueline Maher, de la Direction des échanges de l'OCDE, en a assuré la compilation et la mise en forme. Ce document a été présenté aux séminaires organisés à l'intention des responsables chinois et des ambassades des pays membres de l'OCDE à Beijing, en Chine, les 12 et 13 octobre 2006. 
Le Groupe de travail du Comité des échanges de l'OCDE a examiné le présent rapport et a décidé d'en diffuser plus largement les conclusions en le déclassifiant sous sa responsabilité. L'étude est disponible en anglais et en français sur le site web de l'OCDE : http://www.oecd.org/trade.

\section{Copyright OCDE 2006}

Les demandes d'autorisation de reproduction ou de traduction totale ou partielle de ce document doivent être adressées aux Éditions de l'OCDE, 2 rue André Pascal, 75775 Paris Cedex 16, France. 


\section{TABLE DES MATIERES}

Échanges et croissance de la Chine : incidences sur certains pays de L'OCDE.......................................... 5

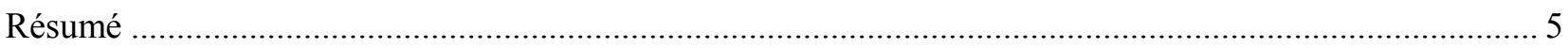

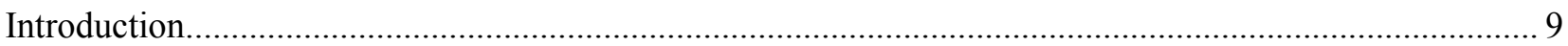

Section I. Cadre de la politique commerciale de la Chine....................................................................... 9

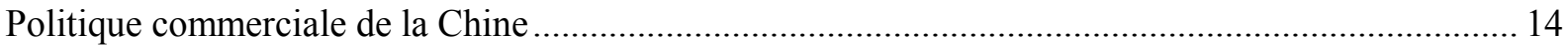

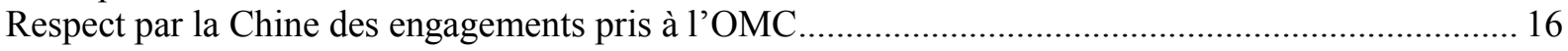

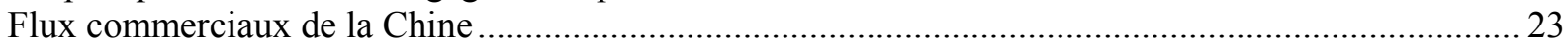

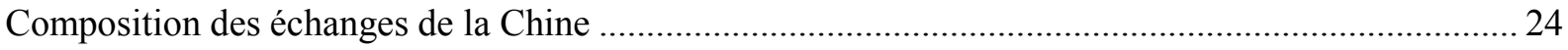

Section II. Impact de la Chine sur l'économie mondiale........................................................................2 26

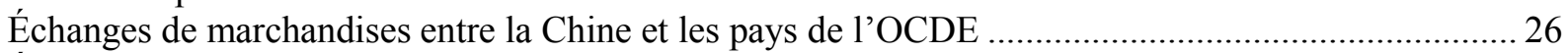

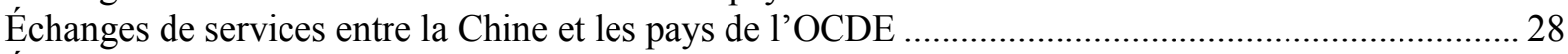

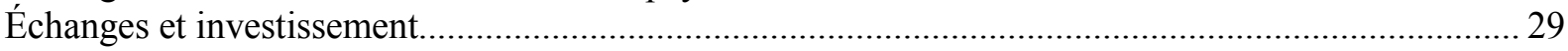

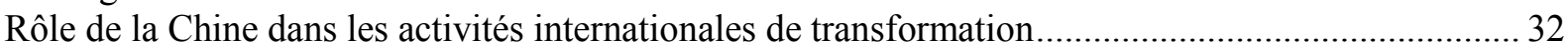

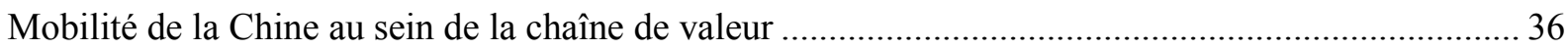

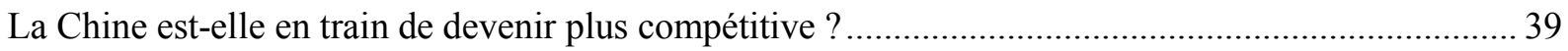

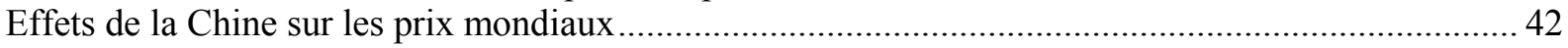

Les termes de l'échange de la Chine et leur effet sur l'économie mondiale .........................................46

Section III. Impact de l'intégration de la Chine sur certaines économies de l'OCDE, une évaluation

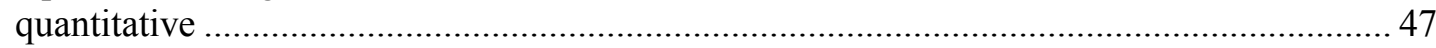

Modélisation de l'impact de l'intégration de la Chine sur les économies de l'OCDE .......................... 47

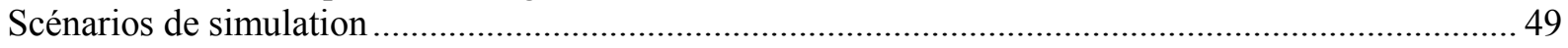

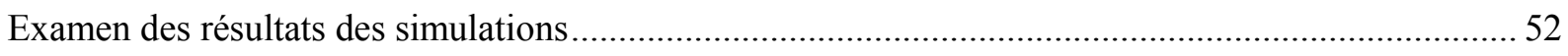

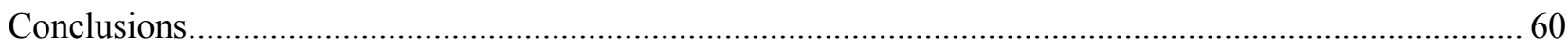

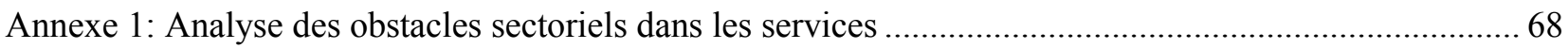

Annexe 2: Modélisation de l'impact de l'intégration de la Chine dans l'économie mondiale, tour d'horizon

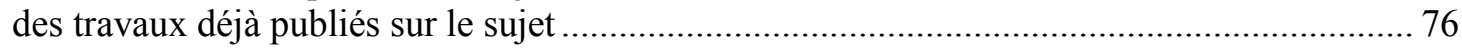

Annexe 3: Structure des échanges et des flux d'investissement direct étranger entre la chine et les pays de

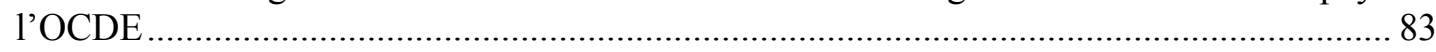

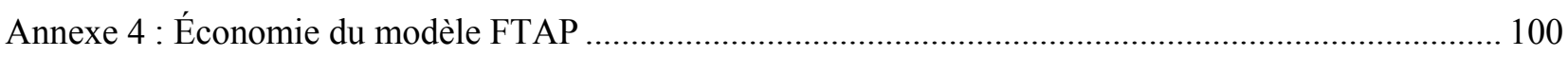

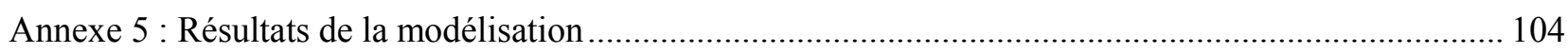


TD/TC/WP(2006)10/FINAL

\section{ÉCHANGES ET CROISSANCE DE LA CHINE : INCIDENCES SUR CERTAINS PAYS DE L'OCDE}

\section{Résumé}

La transformation économique de la Chine est l'une des évolutions économiques les plus spectaculaires de ces dernières décennies. De fait, de 1979 à 2005, le taux de croissance de la Chine s'est en moyenne élevé à $9.6 \%$ par an, et l'intégration du pays dans le système commercial mondial a été tout aussi remarquable : sa part dans les échanges mondiaux de marchandises est passée de moins de $1 \%$ en 1979 à $6.4 \%$ en 2005. La Chine est devenue en 2005 la troisième puissance commerciale au monde après les États-Unis et l'Allemagne. Ses principaux partenaires commerciaux sont, en ordre décroissant, l'Union européenne, les États-Unis et le Japon. À eux trois, ils ont absorbé plus de 51\% de l'ensemble des exportations chinoises en 2005 et représenté près de $34 \%$ de la facture d'importation de la Chine.

L'expansion du commerce extérieur de la Chine a été la clé de sa prééminence croissante dans l'économie mondiale, avec des taux de croissance annuelle des échanges trois fois supérieurs à la moyenne mondiale. L'économie chinoise possède de bons atouts pour devenir le premier exportateur mondial au début de la prochaine décennie (OCDE, 2005a), du fait de son attractivité pour l'IDE, d'un taux élevé d'épargne intérieure, de gains de productivité stimulés par la réduction des obstacles internes et externes au commerce et d'un important excédent de main-d'œuvre. Cependant, comme le note l'OCDE (2005a et 2006b), il lui faudra s'attaquer à un certain nombre de faiblesses structurelles, institutionnelles et macroéconomiques avant de pouvoir réaliser son potentiel. Une libéralisation commerciale plus poussée permettra à la Chine d'échanger des biens et services aux prix mondiaux du marché et, ainsi, d'ajuster sa structure économique de manière à tirer profit de ses véritables avantages comparatifs et à maximiser le bien-être de sa population. Pour tirer le meilleur parti de ces perspectives, la Chine devra revoir certains aspects de sa politique intérieure qui exercent encore une influence notable sur l'activité économique du pays : mesures de contrôle des prix, participation de l'État, subventionnement explicite et implicite, restrictions à la circulation des facteurs de production, etc.

L'expansion du commerce extérieur de la Chine reflète en partie une plus grande spécialisation de la production au sein du continent asiatique. La Chine s'est imposée en tant que plateforme de transformation et d'assemblage finals pour d'énormes volumes d'exportations provenant de ses voisins asiatiques membres de l'OCDE mais destinés aux marchés d'Europe et d'Amérique du nord. Près de la moitié des exportations chinoises entrent dans un tel commerce "triangulaire". Il en est résulté une évolution des relations commerciales bilatérales de la Chine, qui font désormais apparaître des excédents commerciaux croissants avec l'Europe et l'Amérique du nord et des déficits de plus en plus importants avec de nombreux pays d'Asie.

Les activités de transformation de la Chine se sont diversifiées au fil des années : d'abord centrées sur les produits à forte intensité de main-d'œuvre et de basse technologie, elles se sont ensuite étendues à des produits de plus haute technologie. Bien que ses exportations demeurent pour une large part composées de produits à forte intensité de main-d'œuvre, la Chine a élargi sa gamme ces 15 dernières années et exporte aussi désormais des biens complexes à forte intensité de capital et de technologie. Le présent document défend de fait l'idée que la Chine poursuit une stratégie d'expansion de ses exportations sur deux fronts. Le premier aspect de cette stratégie consiste à tirer parti du facteur de production qui constitue son atout majeur - sa main-d'œuvre surabondante - en privilégiant les produits manufacturés à forte intensité de main-d'œuvre, qui créent des emplois. Le second consiste à promouvoir son objectif de développement économique en produisant et en exportant des biens de plus haute technologie. Le pays semble par ailleurs 
s'élever dans la chaîne de valeur : il est devenu en 2004 le premier exportateur mondial de produits des technologies de l'information et de la communication.

La Chine a aussi un impact sur les prix mondiaux. Du fait de son rapide processus d'urbanisation, d'industrialisation et de construction d'infrastructures, le pays importe des quantités toujours croissantes de matières premières et de produits primaires, d'où des pressions à la hausse sur les prix mondiaux des principaux produits de base. Dans le même temps, l'abaissement des obstacles au commerce et la rapide augmentation de la productivité dans le secteur manufacturier et dans celui de l'électronique ont abouti à une soudaine explosion des exportations chinoises, d'où une baisse de leur prix sur les marchés internationaux. Cette tendance perdurera sans doute tant que la Chine poursuivra son processus de "rattrapage". L'ampleur de cette dégradation des termes de l'échange de la Chine est cependant relativement modeste par rapport à la croissance globale de son PIB par habitant. Elle a eu pour contrepartie une augmentation des revenus et des niveaux de vie dans le reste du monde. En effet, l'importation à bas prix de produits manufacturés chinois a accru de près de $0.2 \%$ par an le revenu réel des consommateurs dans la zone OCDE. Par ailleurs, l'augmentation des prix des produits de base non pétroliers n'aura probablement qu'un impact limité sur les revenus dans les pays de l'OCDE, vu que des réponses du côté de l'offre se profilent déjà à l'horizon.

Dans le domaine du commerce des services, les résultats de la Chine contrastent avec ceux obtenus en matière d'échanges de marchandises, en raison de déséquilibres structurels et institutionnels. Les échanges de services de la Chine représentent moins de $10 \%$ de ses échanges totaux et ils se sont soldés par des déficits nets tout au long de la période 1992-2005. De plus, les relations de la Chine avec les pays de l'OCDE comme avec les autres grandes nations commerciales demeurent très limitées dans le domaine des échanges de services, sauf pour ce qui est de ses voisins asiatiques et de l'Australie. Il en va de même pour l'IDE dans le secteur des services : les investissements des pays de l'OCDE en Chine représentent une très faible part de leur stock total d'investissements directs à l'étranger. Les voisins asiatiques de la Chine constituent ses principales sources d'IDE, au nombre desquelles figurent également un certain nombre de paradis fiscaux.

Ces tendances reflètent la dualité de l'économie chinoise : l'ouverture des échanges et de l'IDE dans le secteur des produits manufacturés a favorisé l'émergence d'un secteur pour une large part privé, qui contraste fortement avec le haut niveau de propriété publique et l'importance des obstacles réglementaires qui continuent de caractériser le secteur des services. Les activités de services restent entravées par des obstacles élevés à l'entrée, une participation excessive de l'État, un processus réglementaire opaque et des conditions d'agrément et d'exploitation trop contraignantes. La mise en œuvre intégrale des engagements pris dans le cadre de l'AGCS impliquerait des réformes et des mesures de libéralisation de grande envergure qui procureraient des avantages considérables à la Chine comme à bon nombre de ses partenaires commerciaux. Il convient de noter que le $11^{\text {ème }}$ plan quinquennal met pour la première fois l'accent sur le développement du secteur des services. D'après certaines observations officielles le sousdéveloppement de ce secteur a eu "une incidence négative sur la structure globale de l'industrie, les possibilités d'emploi et la compétitivité d'ensemble."1

Un des points épineux qui ont suscité une grande attention a trait à la protection par la Chine des droits de propriété intellectuelle (DPI). La situation actuelle à cet égard demeure mitigée, opinion dont se sont fait l'écho diverses parties intéressées des milieux gouvernementaux, de l'industrie nationale et des entreprises multinationales. La plupart des données font apparaître de considérables progrès dans la mise en place d'un régime de DPI adapté aux besoins d'une économie de marché moderne. Subsistent toutefois des déficiences de taille - en particulier en ce qui concerne le contrôle de l'application de la

1 China Internet Information Center (2006), Key Points of the 11th Five-Year Guidelines, http://www.china.org.cn/english/2006/Mar/160397.htm, mars 2006. 
réglementation - qui portent préjudice aux intérêts nationaux et internationaux. Compte tenu de l'échelle de l'économie chinoise, ces insuffisances risquent d'avoir des répercussions mondiales.

Les réformes commerciales engagées par la Chine par suite de son accession à l'OMC s'inscrivent dans le prolongement d'une tendance de longue date à la réduction durable des obstacles non tarifaires ainsi que des niveaux et de la dispersion des droits de douane. Les engagements pris par la Chine dans le domaine des services marquent cependant un tournant majeur. Il s'agit notamment de l'ouverture des principaux secteurs de services à la participation étrangère ainsi que de la suppression des restrictions géographiques, aux formes d'établissement et au champ d'activité des entreprises. Quelles sont les conséquences de ces réformes pour la Chine et pour les pays de l'OCDE ? Sur la base des données de 2001, le présent document fournit quelques estimations établies à l'aide d'un modèle d'équilibre général calculable de l'économie mondiale qui tient compte d'une multiplicité de pays et de secteurs, partant de l'hypothèse de rendements d'échelle croissants et d'une situation de concurrence monopolistique entre de grands groupes. Il importe de noter que, dans ce modèle, l'investissement direct étranger est traité sur une base bilatérale, ce qui est essentiel pour comprendre les conséquences de la libéralisation de l'économie chinoise, vu l'importance qu'y revêt la présence étrangère.

Nos résultats montrent que la Chine elle-même a, à l'évidence, beaucoup à gagner à la libéralisation de son économie. D'après les estimations, la mise en œuvre des engagements pris à l'OMC dans les secteurs des biens et des services augmentera de près de $2 \%$ son revenu réel, tandis qu'un scénario de libéralisation complète l'accroîtrait de 3\%. Ces gains résultent en majeure partie d'une utilisation plus efficiente des ressources en Chine.

Nous parvenons à la conclusion que la mise en œuvre par la Chine des engagements pris à l'OMC et une libéralisation complète dans le domaine des droits de douane et des obstacles aux échanges de services auront un impact limité sur les économies de l'OCDE. La structure des échanges bilatéraux entre la Chine et les différentes économies de l'OCDE reflète des dissemblances dans la répartition des avantages comparatifs ainsi que des différences dans la structure des obstacles au commerce et dans la localisation géographique. L'incidence la plus directe résultera probablement d'une amélioration des résultats à l'exportation des pays de l'OCDE qui commercent déjà avec la Chine ou y investissent massivement mais qui se heurtent toujours à d'importants obstacles du point de vue de l'accès au marché. Au vu de la configuration des échanges, l'impact lié à l'accès au marché sera probablement plus sensible pour la Corée, le Japon, l'Australie et la Nouvelle-Zélande, mais demeurera sans doute limité en ce qui concerne les autres économies de l'OCDE.

La libéralisation de l'économie chinoise pourrait également avoir une incidence sur les économies de l'OCDE du fait du surcroît de compétitivité des exportateurs chinois, qui devraient bénéficier d'une baisse des coûts des produits intermédiaires et des services. Les parts de marché non négligeables de la Chine dans les importations des pays de l'OCDE laissent penser que la concurrence accrue des importations constitue assurément une importante conséquence de la libéralisation en Chine. Cependant, ces effets liés à la compétitivité, ressentis aussi bien sur les marchés intérieurs des pays de l'OCDE que sur les marchés des pays tiers, seront presque toujours compensés par les effets liés à l'accès aux marchés (en raison d'un meilleur accès au marché chinois), ce qui procurera dans la majorité des cas, des gains nets globaux aux pays de l'OCDE.

Enfin, les effets liés à l'IDE sont importants car ils l'emportent sur les modestes gains de bien-être de la plupart des pays de l'OCDE dans les scénarios de libéralisation des services. La Chine subira des pertes sur ses investissements directs à l'étranger, tandis que la plupart des pays de l'OCDE tireront des revenus accrus de leurs investissements en Chine. 
Les scénarios fondés sur l'hypothèse de la suppression par la Chine de la totalité de ses droits à l'importation et de ses obstacles aux échanges de services aboutissent à un accroissement des gains mondiaux de un point de pourcentage plus élevé que dans le scénario de l'accession à l'OMC. Cela parait indiquer que les engagements pris par la Chine à l'OMC dans le domaine des droits de douane sur les marchandises et des obstacles aux échanges de services sont déjà très ambitieux et sont à l'origine de l'essentiel des gains que l'on peut attendre de ces réformes. Quoi qu'il en soit, le scénario de libéralisation plus complète procure à la plupart des pays de l'OCDE de plus grands gains tant en termes absolus que par habitant.

Il importe de noter que nos résultats dépendent de données sur la production, la consommation, les échanges et l'investissement qui datent de l'accession de la Chine à l'OMC et qu'ils risquent donc de n'être qu'approximatifs du fait de la rapidité de l'évolution structurelle de l'économie chinoise et des relations entre la Chine et ses partenaires commerciaux de l'OCDE.

Nos résultats concordent aussi, dans l'ensemble, avec les études existantes et, plus fondamentalement, avec les données commerciales sous-jacentes. Par habitant, les pays qui gagnent le plus à la mise en œuvre par la Chine des engagements prix à l'OMC sont la Corée, le Japon, l'UE-15, le Canada et les États-Unis. Tous les pays de l'OCDE gagnants profitent des améliorations de l'efficience allocative, de considérables effets favorables sur leurs termes de l'échange et de revenus accrus tirés de l'IDE dans le secteur des services en Chine. Il convient toutefois de noter que notre analyse ne tient pas compte des effets dynamiques de l'ouverture de la Chine et aboutit donc probablement à des estimations basses. 


\section{Introduction}

1. L'ouverture de la Chine aux échanges mondiaux au cours du dernier quart de siècle a été une des caractéristiques les plus remarquables de la réforme économique et de la mutation structurelle du pays. Autrefois centralement planifiée, l'économie chinoise est désormais plus tournée vers le marché grâce à une transition graduelle extrêmement maîtrisée. À l'issue de 15 années de négociations, la Chine est devenue le $143^{\text {ème }}$ membre de l'OMC le 11 décembre 2001. Son accession à l'OMC marquait symboliquement son intégration en cours à l'économie mondiale et a offert à la Chine comme à ses partenaires commerciaux un accès aux marchés plus sûr et plus prévisible. Fait important, l'accession à l'OMC peut également apparaître comme un effort pour mettre en œuvre des réformes à l'intérieur des frontières du pays, puisqu'elle permettait aux décideurs de mettre en œuvre des politiques économiques visant à poursuivre la libéralisation et l'intégration mondiale des marchés.

2. Au cours des deux dernières décennies, les exportations et les importations chinoises ont spectaculairement augmenté, tout comme la part de la Chine dans les échanges mondiaux. La Chine est un important moteur de croissance de l'économie mondiale, elle stimule aussi bien l'offre que la demande mondiales et bon nombre de ses industries sont totalement intégrées dans la chaîne d'approvisionnement mondiale. La Chine est une des principales plateformes mondiales de production et un des plus vastes marchés émergents de la planète, contribuant à ce titre à la genèse d'une économie vraiment mondialisée. Pays le plus peuplé de la planète, elle possède une des économies qui enregistrent la plus forte croissance au monde et est en outre le pays membre de l'OMC doté du plus vaste marché potentiel. Désormais troisième puissance commerciale au monde, la Chine a développé ses relations avec de nombreux pays membres de l'OCDE. Ses principaux partenaires commerciaux sont l'UE-25, les États-Unis et le Japon. Elle est par ailleurs devenue l'un des principaux partenaires commerciaux d'une majorité de pays de l'OCDE. La Chine s'est imposée comme un acteur majeur sur les marchés internationaux, c'est pourquoi tant les pays de l'OCDE que ceux en développement s'intéressent de plus en plus à son impact potentiel sur les marchés mondiaux.

3. Le présent document examine l'intégration accrue de la Chine dans les échanges internationaux et ses effets sur certains pays de l'OCDE. La première section se penche sur le cadre dans lequel s'est inscrite la politique commerciale de la Chine au cours des 25 dernières années. Elle décrit l'état d'avancement de la libéralisation des échanges, le respect des engagements pris à l'OMC, ainsi que les flux commerciaux de la Chine et leur composition. La deuxième section analyse l'impact de la Chine sur l'économie mondiale. Elle étudie ses échanges de biens et services avec les pays de l'OCDE, ses politiques en matière d'échanges et d'investissement, son rôle dans les activités internationales de transformation et son élévation dans la chaîne de valeur mondiale, en mettant particulièrement l'accent sur l'impact qui en résulte sur les prix mondiaux. La troisième section a recours à un modèle d'équilibre général calculable pour évaluer les effets sectoriels que l'intégration de la Chine dans les échanges mondiaux de biens et de services exerce sur les volumes de production comme sur les prix, ainsi que les répercussions qui s'ensuivent sur le bien-être dans les pays de l'OCDE.

\section{Section I. Cadre de la politique commerciale de la Chine}

4. La Chine a engagé voici à peine plus de 25 ans des réformes économiques graduelles et cumulatives qui ont facilité la transition d'une économie centralement planifiée à une économie plus tournée vers le marché. Le gouvernement s'est rendu compte que, pour assurer cette transition, il serait nécessaire de favoriser l'accès aux capitaux et aux technologies de pointe provenant de l'étranger grâce à une plus grande intégration dans le système commercial multilatéral. En juillet 1986, la Chine a présenté 
une demande officielle d'adhésion au GATT, ${ }^{2}$ mais il lui a fallu 15 années de négociations avant de devenir le 11 décembre 2001 le $143^{\text {ème }}$ pays membre de l'organisation qui a pris la succession du GATT, à savoir l'OMC. L'accession de la Chine à l'OMC marquait symboliquement son intégration en cours dans l'économie mondiale et a offert à la Chine comme à ses partenaires commerciaux un accès aux marchés plus sûr et plus prévisible. On peut toutefois également considérer que l'accession à l'OMC a exercé une influence positive sur les réformes des institutions et des politiques internes au pays. Pour se conformer aux obligations contractées dans le cadre de l'OMC, aussi bien aux frontières du pays qu'à l'intérieur de celles-ci, la Chine doit adopter les disciplines et les principes fondamentaux qui favorisent la mise en œuvre de politiques économiques et réglementaires satisfaisantes. Les accords de l'OMC mettent l'accent sur les engagements contraignants et exécutoires et apportent par là-même un soutien aux réformateurs nationaux, garantissant ainsi que les politiques économiques poursuivent la libéralisation et l'intégration mondiale des marchés.

5. La Chine est aujourd'hui la troisième puissance commerciale mondiale. Pays le plus peuplé de la planète, elle possède l'une des économies qui enregistrent la plus forte croissance au monde et est en outre le pays membre de l'OMC doté du plus vaste marché potentiel. De 1979 à 2005, la croissance économique a atteint en moyenne $9.6 \%$ par an et il semble probable qu'elle se poursuive à ce rythme pendant un certain temps. ${ }^{3}$ L'ouvrage Études économiques de l'OCDE. Chine (2005a) affirme que cette augmentation de la production représente une des transformations économiques les plus rapides et soutenues qui aient été observées au sein de l'économie mondiale au cours des 50 dernières années. L'économie chinoise se classe désormais au second rang derrière celle des États-Unis, si l'on prend pour critère la taille du PIB mesuré en parité des pouvoirs d'achat. ${ }^{4}$

6. Cette rapide croissance économique est en grande partie due aux réformes apportées aux politiques gouvernementales qui ont créé une "économie socialiste de marché" - c'est-à-dire une économie de marché associée à la propriété publique des institutions économiques clés - au sein de laquelle le secteur privé joue un rôle essentiel. ${ }^{5}$ Ces réformes ont débuté par la transformation du secteur agricole et se sont progressivement étendues ces toutes dernières décennies aux industries manufacturières et à de larges pans des secteurs de services. Le tableau 1 examine la composition du PIB au cours des deux dernières décennies. Bien que sa part dans le PIB ait tendance à diminuer, l'agriculture est le secteur qui contribue le plus à l'emploi (44\% en 2002), ce qui donne à penser que la productivité du travail y est bien moindre que dans tous les autres domaine d'activité au sein de l'économie. Compte tenu de la récente révision des chiffres du PIB, ${ }^{6}$ la part des industries manufacturières et des services est encore plus grande. Des

2 La Chine a pris part à la Conférence de Genève qui a conduit à l'adoption du GATT et elle est devenue partie contractante par la signature du Protocole d'application provisoire le 21 avril 1948. Après s'être réfugié dans le Taipei chinois, le gouvernement nationaliste s'est retiré du GATT en mars 1950. La validité de ce retrait était un obstacle à la proposition de la Chine de reprendre son statut de partie contractante. En 1971, la Chine a eu la possibilité de réintégrer le GATT mais a préféré s'abstenir pour diverses raisons d'ordre interne et de nature idéologique. Y. Wang et G. Wang (2005), "China", The World Trade Organization: Legal, Economic and Political Analysis, OMC, Genève : Springer, 2005, vol. 111, pp. 49-50.

3 OCDE (2005a) Études économiques de l'OCDE. Chine, OCDE, Paris, 2005, p. 15. Le 10 janvier 2006, le Bureau national des statistiques de la Chine a revu à la hausse les taux de croissance du PIB chinois.

4 OCDE (2005k) Perspectives économiques de l'OCDE, volume 2005/2, n 78, décembre 2005, p. 4.

5 Pour une étude approfondie de l'économie privée, voir S. Dougherty et R. Herd, (2005) "Fast-Falling Barriers and Growing Concentration: The Emergence of a Private Economy in China", Document de travail du Département des affaires économiques de l'OCDE, n 471, ECO/WKP(2005)58, OCDE, Paris, 16 décembre 2005.

6 En janvier 2006, la Chine a corrigé à la hausse son PIB à la suite d'une révision de la liste des services dont il doit être tenu compte en vue de prendre en considération les petites entreprises, qui appartiennent le plus souvent au secteur privé. Voir OMC (2006) Examen des politiques commerciales - Rapport du Secrétariat - République populaire de Chine, OMC, 2006. 
investissements de grande ampleur financés par une forte épargne intérieure, ainsi qu'une rapide augmentation de la productivité, ont contribué au rythme de la croissance économique. Cependant, bien que ces chiffres montrent que des progrès dans le sens d'une économie axée sur les services commencent à se faire jour en Chine, le pourcentage du PIB imputable aux services et la part de l'emploi dans les secteurs des services sont généralement plus faibles que dans les autres économies en développement (même en tenant compte du récent ajustement à la hausse de la part des services dans le PIB). Cela donne à penser qu'il existe de considérables possibilités d'expansion du secteur des services. La poursuite de la libéralisation des services pourrait contribuer à la mise en œuvre d'ajustements structurels et à l'absorption de main-d'œuvre.

7. Depuis 2000, la contribution du pays à la croissance du PIB mondial (mesurée en parité des pouvoirs d'achat) a été égale à plus de la moitié de la contribution combinée de l'Inde, du Brésil et de la Russie, les trois plus grandes économies émergentes après la Chine. La demande croissante d'importations émanant de la Chine (pour répondre à l'essor de sa demande intérieure et de ses exportations) a constitué une importante source de croissance pour l'économie mondiale. Sa soif de produits de base tels que l'aluminium, l'acier, le cuivre, le charbon et le pétrole a contribué à propulser leurs prix mondiaux à des niveaux records (voir la section II). Dans le même temps, la constante expansion de ses exportations de produits manufacturés exerce une pression à la baisse du prix de ces derniers. De plus, son adhésion à l'OMC en 2001 a accéléré l'ouverture d'un des plus vastes marchés potentiels au monde. La Chine joue également un rôle croissant sur la scène économique mondiale (voir l'encadré 1). La trajectoire de la Chine au cours de la prochaine décennie aura donc à l'évidence d'importantes conséquences pour l'économie mondiale.

Tableau 1. Contributions au PIB, diverses années

(Pourcentage)

\begin{tabular}{|c|c|c|c|c|c|c|c|c|}
\hline Share of main sectors in GDP & 1998 & 1999 & 2000 & 2001 & 2002 & 2003 & 2004 & 2005 \\
\hline Agriculture, forestry and fishing & $\begin{array}{c}18.6 \\
(17.3)\end{array}$ & $\begin{array}{c}17.6 \\
(16.2)\end{array}$ & $\begin{array}{c}16.4 \\
(14.8)\end{array}$ & $\begin{array}{c}15.8 \\
(14.1)\end{array}$ & $\begin{array}{c}15.3 \\
(13.5)\end{array}$ & $\begin{array}{c}14.4 \\
(12.5)\end{array}$ & $\begin{array}{c}15.2 \\
(13.1)\end{array}$ & $\begin{array}{c}\text { na } \\
(12.5)\end{array}$ \\
\hline Industry ${ }^{a}$ & $\begin{array}{c}42.6 \\
(40.3)\end{array}$ & $\begin{array}{c}42.8 \\
(40.0)\end{array}$ & $\begin{array}{c}43.6 \\
(40.3)\end{array}$ & $\begin{array}{l}43.5 \\
(39.8)\end{array}$ & $\begin{array}{c}43.7 \\
(39.4)\end{array}$ & $\begin{array}{c}45.2 \\
(40.5)\end{array}$ & $\begin{array}{c}45.9 \\
(40.8)\end{array}$ & $(47.3)^{b}$ \\
\hline Construction & $\begin{array}{c}6.7 \\
(5.9)\end{array}$ & $\begin{array}{l}6.7 \\
(5.8)\end{array}$ & $\begin{array}{c}6.6 \\
(5.6)\end{array}$ & $\begin{array}{c}6.6 \\
(5.4)\end{array}$ & $\begin{array}{l}6.7 \\
(5.4)\end{array}$ & $\begin{array}{c}7.0 \\
(5.5)\end{array}$ & $\begin{array}{c}7.0 \\
(5.4)\end{array}$ & na \\
\hline Services & $\begin{array}{c}32.1 \\
(36.5)\end{array}$ & $\begin{array}{c}32.9 \\
(38.0)\end{array}$ & $\begin{array}{c}33.4 \\
(39.3)\end{array}$ & $\begin{array}{c}34.1 \\
(40.7)\end{array}$ & $\begin{array}{c}34.3 \\
(41.7)\end{array}$ & $\begin{array}{c}33.4 \\
(41.5)\end{array}$ & $\begin{array}{c}31.9 \\
(40.7) \\
\end{array}$ & $\begin{array}{c}\text { na } \\
(40.3)\end{array}$ \\
\hline
\end{tabular}

a $\mathrm{Y}$ compris les industries extractives, les industries manufacturières, ainsi que la production et la distribution d'électricité, de gaz et d'eau.

b Y compris le secteur de la construction.

Note : Les données entre parenthèses correspondent aux pourcentages par rapport aux chiffres du PIB corrigé, tels qu'ils ont été communiqués le 10 janvier 2006 par le Bureau national des statistiques de la Chine.

Source: OMC (2006), sur la base de l'annuaire statistique 2005 du Bureau national des statistiques de la Chine ; China Labour Statistical Yearbook 2002 ; PNUD, Rapport sur le développement humain (diverses éditions) ; et autorités chinoises. 


\section{Encadré 1. La Chine sur la scène économique mondiale}

L'importance croissante de la Chine au sein de l'économie mondiale a également poussé le pays à prendre une part plus active à l'élaboration des politiques à l'échelle internationale. Les responsables chinois ont compris qu'ils peuvent exercer une influence sur l'élaboration de règles économiques et commerciales multilatérales. Le gouvernement chinois s'est notamment attaché à assumer ce rôle directeur en participant au Groupe des 20 (G-20), à l'OMC et à la Coopération économique pour l'Asie-Pacifique (APEC). ${ }^{7}$ Créé en 1999, le G-20 regroupe les principaux pays industriels et les principales économies de marché émergentes. Il compte pour plus de $90 \%$ du PIB mondial, $80 \%$ du commerce international, et les deux tiers de la population mondiale. La Chine en a assumé la présidence en $2005 .^{8}$ Le pays participe par ailleurs davantage à l'OMC. Il a ainsi accueilli, de sa propre initiative, une réunion informelle à Dalian les 12 et 13 juillet 2005, à laquelle ont assisté les ministres du Commerce de 30 pays membres influents de l'OMC en vue de soutenir les négociations dans le cadre du Programme de Doha pour le développement.

L'APEC a été créée en 1989 pour tirer parti de l'interdépendance croissante des économies de l'Asie-Pacifique. L'APEC s'est depuis lors développée pour devenir l'un des plus importants groupements régionaux au monde. Ses 21 économies membres s'étendent sur quatre continents, abritent plus de 2.6 milliards de personnes et comptent pour $57 \%$ (soit 20.7 billions USD) du PIB mondial et $45.8 \%$ (soit 7 billions USD) des échanges mondiaux. ${ }^{9}$ La Chine, qui en est membre depuis 1991, est activement engagée pour des raisons stratégiques et économiques dans cette organisation qui a pour but de promouvoir les échanges internationaux, et elle a souscrit aux Objectifs de Bogor de libéralisation et d'ouverture des échanges et de l'investissement dans la région Asie-Pacifique. ${ }^{10}$ L'expansion des relations commerciales de la Chine et son interdépendance accrue ne sont nulle part plus évidentes qu'en Asie du sud-est, où elle s'impose rapidement comme un moteur de la croissance et de l'intégration économiques régionales. ${ }^{11}$

Bien que la Chine soit un membre de plus en plus actif de ces organisations, son rôle demeure à l'évidence modeste par rapport à son poids économique et politique. Alors que s'approche la fin de la période de transition dont elle bénéficie en sa qualité de nouveau membre de l'OMC, des appels se sont élevés pour que, en sa qualité de troisième puissance commerciale de la planète, elle assume davantage de responsabilités dans les relations commerciales mondiales. Les États-Unis soutiennent que la Chine s'est manifestement élevée au rang des grandes puissances commerciales mondiales qui, à l'instar des États-Unis, de l'UE et du Japon, ne sont pas seulement tenues de remplir leurs engagements mais doivent également aller au-delà pour préserver le bon fonctionnement du système mondial d'échanges. ${ }^{12}$

7 La question de l'efficacité de la prise de décision au sein du G8 alors que la Chine n'en est pas membre n'est pas sans susciter quelques débats. Voir J. Garten (2004), "China: The Missing Member at the G-8 Table", YaleGlobal Online, 3 juin 2004.

8 Pour plus d'informations, voir www.g20.org/index.htm. Pour un examen approfondi des relations entre le G20 et la Chine sous un angle de vue chinois, voir Y. Yu (2005), "G-20 and China: A Chinese Perspective", China \& World Economy, vol. 13, n 1, 2005.

9 APEC (2005) APEC at a Glance, APEC, 2005, p. 1.

10 Lors de sa $17^{\text {ème }}$ réunion ministérielle qui s'est tenue en Corée en novembre 2005, les ministres ont déclaré qu'ils demeurent totalement attachés à la réalisation des Objectifs de Bogor de libéralisation et d'ouverture des échanges et de l'investissement en Asie-Pacifique dès 2010 pour les pays membres développés et d'ici 2020 pour les pays membres en développement, conformément à ce que prévoit la Déclaration de Bogor. Voir www.apecsec.org.sg/apec/ministerial_statements/annual_ministerial/2005_17th_apec_ministerial.html

11 Dans le cadre de sa stratégie d'“ascension pacifique" en Asie du Sud-Est, la Chine a recours aux échanges commerciaux, à l'aide au développement du Laos, du Myanmar et du Cambodge, ainsi qu'à des mesures de renforcement de la confiance pour s'imposer comme un important chef de file au sein de la région. Voir E. Economy (2005), “China's Rise in Southeast Asia: implications for the US”, Journal of Contemporary China, 14(44), aôut 2005.

12 Voir USTR (2006), U.S.-China Trade Relations: Entering a New Phase of Greater Accountability and Enforcement, Top-to-Bottom Review, USTR, Washington, DC, février 2006, p. 11. 
8. Pour maintenir le rythme de croissance actuel, le gouvernement chinois devra poursuivre ses efforts de réforme. L'OCDE (2005a) a établi que des réformes sont nécessaires dans plusieurs domaines. ${ }^{13}$ Il s'agit tout d'abord d'assurer un environnement stable aux activités du secteur privé. La restructuration du secteur des entreprises d'État doit se poursuivre, et l'ampleur doit encore en être réduite. Les performances du secteur des entreprises pourraient être améliorées moyennant une plus grande modernisation de l'environnement dans lequel elles opèrent et une meilleure application des lois, et notamment de celles relatives aux droits de propriété intellectuelle (voir ci-dessous). Il conviendrait en second lieu de mettre en place un régime de change plus souple qui favoriserait la stabilité de l'environnement macroéconomique. Les modifications du régime de change annoncées en juillet 2005 sont un pas dans la bonne direction. ${ }^{14}$ Troisièmement, il faudra réformer le système financier. Bien qu'il ait été recapitalisé à environ $70 \%$, le système bancaire chinois demeure accablé par une quantité considérable de créances irrécouvrables détenues par les banques contrôlées par l'État. ${ }^{15}$ La capacité de celles-ci à soutenir la concurrence des banques étrangères qui seront autorisées à opérer en Chine conformément aux engagements pris lors de l'adhésion à l'OMC s'en trouvera amoindrie. Il est nécessaire d'améliorer la gouvernance et de développer la propriété privée.

9. La croissance, les perspectives en matière de commerce extérieur et l'investissement se concentrent dans les régions déjà prospères du littoral et ne bénéficient que bien trop peu aux régions de l'intérieur et de l'ouest du pays. Il conviendrait donc de réduire les inégalités régionales en procédant à des réformes dans un quatrième domaine, à savoir celui des transferts budgétaires. Des finances publiques solides donneraient les moyens de procéder à des réformes du système fiscal et des dépenses budgétaires. Les dépenses dans le domaine de la santé et de l'éducation pourraient être augmentées. Une urbanisation rapide et accrue est recommandée. L'économie chinoise devra relever un cinquième défi à long terme en faisant face à la dégradation de l'environnement. ${ }^{16}$ Une récente étude sur la gouvernance en Chine (OCDE, 2005c) examine un sixième domaine de réforme : s'il veut que la Chine bénéficie d'un développement durable, le gouvernement devra entreprendre un vaste programme de réformes institutionnelles de grande ampleur. Certains observateurs internationaux ont également fait part de leurs craintes concernant la croissance rapide de l'économie chinoise, sa forte consommation d'énergie et le large écart entre les riches et les pauvres.

10. La Chine paraît avoir conscience des défis qu'elle doit relever. Le $11^{\text {ème }}$ Plan quinquennal (20062010) marque une importante réorientation des politiques du gouvernement chinois. Il vise à améliorer la structure économique, la capacité d'innovation et le mode de croissance du pays, à maîtriser la pollution, créer davantage d'emplois, établir un équilibre entre l'investissement et la consommation, réduire les écarts de revenus et promouvoir le bien-être public. Le Plan souligne que "le développement économique est la

13 Pour une étude approfondie de l'économie chinoise et des défis qu'elle devra relever à l'avenir, voir OCDE, (2005a), op. cit. et OCDE (2006b), Les dépenses publiques en Chine: Vers plus d'efficacité et d'équité, OCDE, Paris 2006.

$14 \quad$ La Chine a laissé sa monnaie s'apprécier de 2.1\% le 21 juillet 2005.

15 L'agence de notation Standard \& Poor's estime que les banques chinoises possèdent près de 650 milliards de créances irrécouvrables, ce qui représente $40 \%$ de l'encours total des prêts. Martin Wolf soutient que si la Chine enregistre une croissance de près de $10 \%$ par an et génère des créances irrécouvrables d'une telle ampleur, la mauvaise allocation des ressources en capital doit être gigantesque. Voir M. Wolf, "Why is China is Growing so Slowly?", Foreign Policy Special Report, Carnegie Endowment for International Peace, janvier/février 2005, p. 51.

16 La Banque mondiale affirme que l'air et l'eau sont très pollués en Chine. Sept des dix villes les plus polluées au monde à la fin des années 90 étaient situées en Chine. Un tiers des prairies chinoises, qui couvrent $40 \% \mathrm{du}$ territoire du pays, sont sérieusement dégradées. Banque mondiale, (2004), China: An Evaluation of World Bank Assistance, 2004, p. 3. l'OCDE a commencé à travailler à un Examen des performances environnementales de la Chine qui sera achevé fin de 2006. 
toute première priorité", mais qu'il devrait être "général, harmonieux et durable". Cela implique "une croissance économique stable et relativement rapide", ainsi que la nécessité d'“accélérer la transformation du mode de croissance économique" au profit d'une croissance exigeant de moindres proportions d'énergie, de ressources et de capitaux, davantage axée sur le savoir et l'innovation et plus équitablement partagée. ${ }^{17}$ Chose importante, le plan de développement économique et social de la Chine considère qu'il est essentiel de créer une société modérément prospère sur l'ensemble du territoire du pays en mettant en œuvre une politique respectueuse de l'environnement et axée sur une croissance et un développement durables.

\section{Politique commerciale de la Chine}

11. L'ouverture de la Chine au commerce mondial au cours du dernier quart de siècle a été une des caractéristiques les plus remarquables de la réforme économique et de la mutation structurelle du pays. La Chine est passée de l'autarcie (auto-approvisionnement) à une économie plus ouverte grâce à une transition graduelle extrêmement maîtrisée. La Chine a engagé le processus d'ouverture progressive de son marché en autorisant les activités de transformation pour l'exportation menées à petite échelle. Dès 1978, la Chine a commencé à autoriser les entreprises de Hong Kong à proposer des contrats de transformation pour l'exportation aux ateliers de la province de Guangdong. Au départ, les activités de transformation pour l'exportation étaient dans une large mesure limitées à un petit nombre de zones franches agréées spécialement destinées à l'industrie d'exportation et situées le long de la côte méridionale de la Chine, mais elles se sont largement répandues au milieu des années 80 . Les différentes localités se sont livrées une concurrence acharnée pour attirer les investissements dans les activités de transformation pour l'exportation. Les zones franches d'exportation ont permis à la Chine d'exploiter rapidement son avantage comparatif pour ce qui est de la main-d'œuvre à bas salaires.

12. À mesure que les producteurs ont pris pied sur les marchés mondiaux, les activités de transformation pour l'exportation se sont sensiblement développées, favorisées par l'appréciation des monnaies des nouvelles économies industrielles (NEI) d'Asie voisines. Les plus fortes incitations à fragmenter la production à la recherche d'une main-d'œuvre aux salaires plus bas ont également été à l'origine d'une augmentation des investissements en Chine. Aussi cette économie auparavant fermée est-elle désormais de plus en plus intégrée dans les chaînes de production dynamiques d'Asie orientale. Dès le milieu des années 80 , la Chine s'était dotée d'un régime d'exportation à deux vitesses établissant une claire distinction entre un segment très ouvert axé sur les activités de transformation pour l'exportation et un secteur d'exportation purement national auquel étaient accordés de hauts degrés de protection à l'intérieur du pays. Le second ne pouvait notamment pas bénéficier d'importations en franchise de droits. Le marché intérieur était protégé par de hauts niveaux de protection au travers des droits de douane et de plusieurs strates d'obstacles non tarifaires.

13. Lorsqu'elle a demandé à adhérer au GATT en 1986, la Chine était pour l'essentiel une économie centralement planifiée dotée d'un régime commercial opaque caractérisé par des droits de douane élevés et par une pléthore d'obstacles non tarifaires. Elle avait pour principaux partenaires commerciaux des pays socialistes tels que l'URSS et la Yougoslavie. Ce n'est qu'en 1992, lorsqu'elle a déclaré son intention de créer une "économie socialiste de marché", que la Chine a commencé à baisser ses droits de douane. La Chine a alors unilatéralement commencé à appliquer de sensibles réductions tarifaires. Comme le montre le tableau 2, l'abaissement des droits de douane au cours des années 90 a été tel que la Chine était sans doute l'une des économies en développement les plus ouvertes à adhérer à l'OMC en 2001. Le taux moyen simple des droits de douane appliqués par la Chine a été ramené de $42.9 \%$ en 1992 à $16.6 \%$ en 2001. Après

$17 \quad$ Voir le Communiqué de la $5^{\text {ème }}$ session plénière de $16^{\text {ème }}$ Comité central du Parti communiste chinois, 11 octobre 2005. 
l'accession à l'OMC, il est tombé à 9.8\%. Au-delà de l'ouverture de ses marchés à ses partenaires commerciaux, cette réduction a induit d'importants gains d'efficience et de productivité en Chine.

Tableau 2. Chine : Droits de douane officiels simples et pondérés en fonction des échanges, diverses années

(Pourcentage)

\begin{tabular}{lcccccc}
\hline & \multicolumn{2}{c}{ All Products } & \multicolumn{2}{c}{ Primary Products } & \multicolumn{2}{c}{ Manufactures } \\
\hline & Simple & Weighted & Simple & Weighted & Simple & Weighted \\
1992 & 42.9 & 40.6 & 36.2 & 22.3 & 44.6 & 46.5 \\
1996 & 23.6 & 22.6 & 25.4 & 20.0 & 23.1 & 23.2 \\
2001 & 16.6 & 12.0 & 21.6 & 17.7 & 16.2 & 13.0 \\
After accession & 9.8 & 6.8 & 13.2 & 3.6 & 9.5 & 6.9 \\
\hline
\end{tabular}

Source: Données de la Banque mondiale et de la Base de données statistiques de l'ONU sur le commerce de marchandises (COMTRADE) telles que citées in Banque mondiale (2004), tableau 13.2.

14. Dans le cadre du processus d'accession à l'OMC, la Chine a accepté de mettre en œuvre une série de réformes radicales qui impliquaient un abaissement des barrières commerciales dans presque tous les secteurs de son économie, l'application du traitement national et l'assurance d'un meilleur accès à ses marchés des biens et services, la protection des droits de propriété intellectuelle, une plus grande transparence, ainsi que l'élimination des obstacles non tarifaires. La Chine a également accepté des règles spéciales relatives aux subventions et à l'activité des entreprises d'État, compte tenu du rôle de l'État dans l'économie chinoise. ${ }^{18}$ Ces engagements contribueront sans doute grandement à élargir et accélérer le processus de libéralisation des échanges en Chine, à accroître l'efficience économique et les revenus et à favoriser une intégration plus poussée de la Chine dans l'économie mondiale.

15. Une des principales raisons qui ont poussé la Chine à adhérer à l'OMC était son souci d'assurer un environnement commercial sûr et prévisible et d'offrir ainsi un environnement plus viable aux entreprises. L'engagement de la Chine de consolider ses droits de douane a donné aux entreprises une vision plus claire des perspectives qui s'ouvrent à elles. La Chine a consolidé la totalité de ses droits à l'importation de marchandises. Lorsque tous ses engagements en matière d'accès aux marchés auront été mis en œuvre, le niveau moyen des droits de douane consolidés de la Chine sera ramené à $15 \%$ pour les produits agricoles, la fourchette de ces droits allant de 0 à $65 \%$ et les taux les plus élevés étant appliqués aux céréales. Pour ce qui est des biens industriels, le niveau moyen des droits de douane consolidés tombera à $8.9 \%$, la fourchette de ces droits allant de 0 à $47 \%$, les taux les plus élevés étant appliqués aux pellicules photographiques et aux automobiles et produits connexes. ${ }^{19}$ La Chine s'est également engagée à pousser plus loin la réduction et la suppression progressives des obstacles non tarifaires, pour la plupart dès 2005, mais au plus tard en 2010. La Chine a en outre pris l'engagement de limiter ses subventions agricoles à $8.5 \%$ de la valeur de la production du secteur. ${ }^{20}$

18 Pour plus d'informations sur les entreprises d'État, voir OCDE (2005c), "Réformer la gestion des actifs de l'État et améliorer le gouvernement d'entreprise : les deux enjeux majeurs de la réforme des entreprises en Chine", La gouvernance en Chine, OCDE, Paris, 2005, pp. 341-366.

19 OMC (2001a), “Achèvement des négociations sur l'accession de la Chine a l'OMC", Communiqué de presse de l'OMC (Genève, 17 septembre 2001).

20 L'agriculture compte pour près de $15 \%$ du PIB et fournit plus de $40 \%$ de l'emploi en Chine. La tendance à la baisse de la part de l'agriculture dans l'ensemble des échanges est particulièrement frappante. En effet, malgré une augmentation de leur valeur réelle, la part des importations et des exportations agroalimentaires est tombée d'environ 15\% en 1990 à seulement 4\% en 2003. Voir AGR/CA(2005)6, p. 14. Pour une étude détaillée des politiques agricoles de la Chine, voir OCDE (2005f) Review of Agricultural Policies in China, OCDE, Paris, 2005. 
16. Le commerce de services a été un des domaines clés des négociations d'accession de la Chine à l'OMC, qui ont abouti à l'engagement d'ouvrir sensiblement un vaste éventail de secteurs de services grâce à l'élimination de bon nombre des restrictions à l'accès aux marchés existantes. La Chine a accepté d'autoriser les fournisseurs de services étrangers à participer au commerce de détail de tous les produits dès la fin de 2003. Depuis la fin de 2004, toutes les entreprises ont le droit d'importer et d'exporter tous les biens, sauf ceux dont le commerce fait l'objet d'un monopole d'État (comme le pétrole ou les engrais). Les entreprises étrangères sont autorisées à distribuer quasiment tous les biens au sein du pays dès la fin de 2006. Pour ce qui est des opérations en devises, les établissements financiers étrangers sont en droit de fournir dès l'accession des services sans restrictions quant à la clientèle. En ce qui concerne les opérations en monnaie locale, ils pourront fournir des services aux entreprises chinoises dès décembre 2003 et à tous les clients chinois à compter de décembre 2006.

17. Des secteurs essentiels tels que ceux des télécommunications, de la banque et de l'assurance seront désormais confrontés à une concurrence bien plus intense. La Chine s'est engagée à éliminer dès la fin 2006 la plupart des restrictions à l'entrée et à la participation étrangères, ainsi que la plupart des formes de discrimination à l'encontre des entreprises étrangères. Un accès intégral finira par être assuré aux fournisseurs étrangers au moyen de procédures transparentes et automatiques pour l'attribution de licences. Le pays lèvera les restrictions aux échanges et à la distribution dans le pays de nombreux produits. Toutes les conséquences de cette concurrence accrue ne se feront sentir en Chine qu'après qu'ait eu lieu la libéralisation du reste des secteurs conformément au calendrier établi dans le cadre de l'accord de l'OMC.

18. Pour mesurer à quel point la Chine a ouvert son commerce extérieur, il suffit d'examiner l'évolution dans le temps de la part du PIB que représente le volume total des échanges (exportations plus importations). D'après les Indicateurs du développement de la Banque mondiale, la contribution des échanges au PIB est passée de $5.3 \%$ en 1970 à $34.8 \%$ en 1990 et $44.2 \%$ en 2000 , avant de monter en flèche après l'accession à l'OMC pour atteindre le chiffre gigantesque de $65.4 \%$ en 2004 (tableau 3).

Tableau 3. Chine : Part des échanges dans le PIB

(Pourcentage)

\begin{tabular}{ccccccccc}
\hline 1970 & 1975 & 1980 & 1985 & 1990 & 1995 & 2000 & 2003 & 2004 \\
\hline 5.3 & 9.2 & 21.8 & 24.1 & 34.8 & 43.9 & 44.2 & 56.9 & 65.4 \\
\hline
\end{tabular}

Source : Banque mondiale, Base de données des indicateurs du développement dans le monde

19. Le degré auquel la Chine a ouvert son régime commercial ne peut être minimisé, en particulier depuis son adhésion à l'OMC. D'importantes restrictions subsistent cependant dans certains secteurs. Elles seront examinées dans la prochaine section.

\section{Respect par la Chine des engagements pris à l'OMC}

20. Dans le cadre du Protocole d'accession de la Chine, l'OMC a mis en place en vertu de l'article 18 un mécanisme d'examen transitoire pour vérifier chaque année pendant 8 ans si la Chine se conforme à ses engagements, un examen final ayant par la suite lieu la dixième année. ${ }^{21}$ Ce mécanisme exige que la Chine fournisse des informations détaillées sur ses efforts de mise en œuvre et donne à tous les membres de l'OMC la possibilité de soulever des questions dans un cadre multilatéral sur la manière dont la Chine s'acquitte de ses engagements. Il est mis en œuvre devant un certain nombre d'organes subsidiaires de l'OMC, qui présentent un rapport au Conseil général de l'OMC. Le Conseil général procède alors chaque année à un examen d'ensemble et peut formuler des recommandations. Lors de la mise en place de ce mécanisme, l'espoir était que le processus d'examen serait un outil important et efficace qui permettrait de

21 Voir OMC (2001b), Article 18 du "Protocole d'accession de la République de Chine", OMC, WT/L/432, 23 novembre 2001. 
suivre les progrès accomplis par la Chine du point de vue du respect de ses obligations et de ses engagements dans le cadre de l'OMC et qu'il stimulerait la réforme du régime commercial de la Chine.

21. Jusqu'à ce jour, le mécanisme d'examen transitoire n'a pas démontré son efficacité en tant qu'outil permettant de vérifier et de garantir le respect des engagements pris comme le prévoyait le protocole. ${ }^{22}$ Bien que la plupart des pays membres de l'OMC ne fassent pas appel à ce mécanisme, l'UE, le Japon et les États-Unis, ainsi que quelques autres, ont continué à y avoir recours en 2005. Après cinq années, certaines craintes subsistent concernant la mise en œuvre et le respect par la Chine des engagements pris à l'OMC. L'OMC a achevé en avril 2006 son premier examen de la politique commerciale de la Chine. ${ }^{23}$ Cet examen se penche sur les politiques commerciales de la Chine au travers pour l'essentiel d'une évaluation par les pairs.

22. Le mécanisme d'examen transitoire a permis aux pays membres de l'OMC d'identifier un certain nombre de domaines qui soulèvent des problèmes, dont les programmes d'assistance industrielle, les normes, les réglementations techniques et les autres obstacles non tarifaires, mais aussi en particulier l'accès aux marchés pour les services et le respect des droits de propriété intellectuelle (DPI). ${ }^{24}$ Un récent rapport du Représentant des États-Unis pour le commerce extérieur (United States Trade Representative ou USTR) examine ces différents domaines. ${ }^{25}$ Le premier motif de préoccupation tient au fait que la Chine possède des politiques industrielles qui paraissent limiter l'accès aux marchés pour ce qui est des biens d'origine non chinoise ou consacrer d'importantes ressources publiques à l'augmentation de la production et des exportations chinoises. Sont notamment en cause les privilèges dont bénéficient les entreprises d'État, et plus particulièrement les banques d'État. Une autre crainte a trait à la promulgation de normes et autres réglementations techniques qui paraissent favoriser les biens produits à l'intérieur du pays, ainsi qu'à une application discriminatoire des normes au détriment des produits non chinois.

23. Le rapport de l'USTR met l'accent sur deux problèmes particuliers : l'accès aux marchés pour les secteurs de services et le respect des DPI. Bien que la Chine ait jusqu'à la fin 2006 pour mettre en œuvre certains des engagements pris à l'OMC dans le domaine des services, certains problèmes apparaissent en ce qui concerne l'accès aux marchés de services, notamment dans les secteurs des télécommunications, de la distribution et de la construction. Ce rapport constate également que les secteurs des services se heurtent à des obstacles par lesquels les organismes de réglementation chinois continuent de faire échec aux efforts des fournisseurs étrangers grâce à un processus réglementaire opaque et à des conditions d'agrément et d'exploitation trop contraignantes.

24. Le Secrétariat de l'OCDE a également constaté que tel était le cas au travers de l'examen des obstacles aux échanges de services dans les secteurs de la banque, de l'assurance, des télécommunications (fixes et mobiles), des services professionnels (ingénierie) et de la distribution auquel il a procédé. ${ }^{26}$ L'étude en question évaluait les obstacles aux échanges de services ainsi que les effets de leur libéralisation dans de nombreux pays, dont la Chine. À l'aide d'autres méthodes de pondération et de spécifications économétriques améliorées qui prennent en compte les obstacles entravant chaque mode de fourniture de services et d'autres variables réglementaires propres à un secteur particulier, ce document tirait des conclusions concernant le degré de restriction des échanges opposé par la Chine aux pays en

\footnotetext{
22 Pour une évaluation du mécanisme d'examen transitoire, voir W. Steinberg (2006), Monitor with No Teeth: An Analysis of the WTO China Trade Review Mechanism, $1^{\text {er }}$ janvier 2006, http://blj.ucdavis.edu/article/578/.

23 Voir OMC (2006), Examen des politiques commerciales - République populaire de Chine, OMC, avril 2006.

24 M. Overmyer (2006), "WTO: Year Five”, China Business Review, janvier 2006, pp. 5-6, http://www.chinabusinessreview.com/public/0601/overmyer.html.

25 USTR (2006), op. cit.

26 Voir OCDE (2005j) "Modal Estimates of Services Barriers", TD/TC/WP(2005)36, 8 novembre 2005.
} 
développement comme à ceux membres de l'OCDE. Les résultats montrent que, d'après les données de 2004, la Chine appliquait un régime très restrictif dans les secteurs de la banque, de l'assurance, des télécommunications mobiles, de l'ingénierie et de la distribution.

25. Ces résultats sont conformes aux constatations d'ensemble, qui montrent qu'avant la mise en œuvre progressive des engagements de libéralisation inscrits sur sa liste de l'AGCS, le nombre de secteurs où un plein accès était garanti était plus faible en Chine que dans toutes les autres catégories de pays (développés, en développement, et candidats à l'accession). ${ }^{27}$ Comme le fait remarquer Mattoo (2003), le tableau pourrait être radicalement différent après la mise en œuvre des engagements de libéralisation. Dans l'ensemble, les engagements pris dans le domaine de l'accès aux marchés sont d'une ampleur et d'une étendue bien plus grandes que les concessions offertes lors du Cycle d'Uruguay par n'importe quelle autre catégorie de pays (y compris ceux à revenu élevé). Par ailleurs, les engagements de la Chine en matière de traitement national sont de plus grande ampleur et de plus grande étendue que ceux de toutes les autres catégories de pays. L'annexe 1 décrit en détail ces réformes, qui ont été prises pour base pour la définition des scénarios de simulation utilisés par la suite, lesquels correspondent à la mise en œuvre des engagements de la Chine dans ces cinq secteurs de services.

\section{Droits de propriété intellectuelle en Chine}

26. Au fil des décennies, après avoir adhéré à l'Organisation mondiale de la propriété intellectuelle en 1980, la Chine a accompli de considérables progrès dans la mise en place d'un régime de DPI adapté au fonctionnement d'une économie de marché. Bien que les données disponibles montrent que de nouvelles améliorations devraient lui être apportées - en particulier en ce qui concerne l'application de la législation en la matière - il est clair que le système porte déjà ses fruits et encourage l'innovation au sein du pays, ainsi que la diffusion ultérieure des résultats de celle-ci. Par exemple, en 2005, la Chine a déposé 2452 demandes de brevets en vertu du Traité de coopération en matière de brevets (TCB) et a occupé la $10^{\text {ème }}$ place au classement mondial des pays ayant le plus recours au Traité. ${ }^{28}$ Cela représentait une augmentation de $44 \%$ par rapport à l'année précédente et plaçait la Chine devant des pays signataires du Traité tels que le Canada, l'Italie ou l'Australie. Globalement, la Chine comptait pour près de $2 \%$ des demandes déposées en vertu du Traité. Qui plus est, la création d'un meilleur environnement dans le domaine des DPI contribue à encourager un plus grand transfert international de technologies vers la Chine. Les progrès constants observés concernant la mise en place du régime de DPI de la Chine offrent la promesse de nouveaux avantages sous la forme d'un accroissement du stock de technologies disponibles.

\section{L'environnement en matière de protection des DPI en Chine}

27. Le gouvernement chinois a déclaré qu'il considère la protection des DPI comme un élément de sa stratégie visant à encourager un environnement propice aux échanges, à l'investissement et à la recherchedéveloppement en Chine, et non simplement comme "une réponse passive aux engagements pris par la Chine en vue de son accession [...]." ${ }^{, 29}$ Dans le cadre des propositions pour le $11^{\text {ème }}$ Plan quinquennal, le Conseil d'État de la République populaire de Chine a publié le 9 février 2006 des Lignes directrices nationales pour le programme à moyen et à long terme en faveur de la science et de la technologie 20062020 [National guideline on medium- and long-term program for science and technology development (2006-2020)], qui affirment que la Chine a pour projet de devenir un pays innovateur dans les 15 prochaines années et une puissance mondiale dans le domaine des sciences et de la technologie d'ici le

\footnotetext{
27 Voir Mattoo (2003).

28 D'après un article de l'Agence de presse Xinhua ("Chine nouvelle") daté du 6 février. Tous les articles de l'Agence de presse Xinhua ("Chine nouvelle") cités dans le présent document étaient disponibles sur le site www.china.org.cn au 27 février 2006. 
milieu du XXI ${ }^{\text {ème }}$ siècle. ${ }^{30}$ Ces lignes directrices soulignent l'intention de hâter la mise en œuvre d'un système national de DPI garantissant le respect et la protection des droits de propriété intellectuelle tout en évitant l'usage abusif de ceux-ci, qui risquerait de nuire à une concurrence "normale" et à l'innovation.

28. Le 14 mars 2006, l'Office public de la propriété intellectuelle (State Intellectual Property Office) a publié un Plan d'action 2006 pour la protection des DPI en Chine (China's Plan on IPR Protection Action 2006). ${ }^{31}$ Ce plan d'action énonce les priorités en matière de renforcement du système de DPI dans quatre grands domaines : les marques, les droits d'auteur, les brevets et le commerce international (y compris les questions douanières). Le plan prévoit que des mesures seront prises dans neuf domaines: réforme législative, respect de la loi (7 campagnes spécialisées et diverses autres initiatives et mesures spéciales), renforcement des institutions, actions de sensibilisation ("semaine de protection des DPI", brochures, émissions de télévision), formation et éducation, échanges et coopération internationale (par exemple avec les offices de brevets étrangers), promotion de l' "autodiscipline" des entreprises (par exemple en ce qui concerne l'utilisation de logiciels légaux), amélioration des services offerts aux détenteurs de droits (tels que l'accès aux technologies de l'information pour la recherche d'informations sur les brevets) et extension des recherches sur les questions relatives aux DPI en Chine..$^{32}$ Le plan d'action souligne l'intention des autorités de répondre à un certain nombre de préoccupations exprimées par les titulaires de droits étrangers, y compris en ce qui concerne les problèmes d'application de la législation. Il n'indique cependant pas si des ressources supplémentaires seront débloquées dans chacun de ces neuf domaines d'action.

29. L'attention accrue accordée par les autorités aux questions relatives aux DPI dans le cadre du plan quinquennal et du plan d'action en cours est associée à la reconnaissance par le gouvernement de la nécessité de réformer le régime des DPI et d'élaborer une meilleure approche stratégique de la politique en matière de DPI. Une étude officielle du Centre de recherches sur le développement (Development Research Center ou DRC) du Conseil d'État (2004) a examiné la situation du système de DPI chinois. Les conclusions auxquelles elle parvient montrent que le système a enregistré un rapide développement en 20 ans et s'avère "relativement complet" du point de vue des aspects juridiques couverts. Le rapport souligne à quel point le système de DPI contribue à encourager l'investissement étranger et surtout l'innovation des entreprises locales. Il reconnaît dans le même temps la nécessité de renforcer la mise en œuvre des règles régissant les DPI. Les principaux obstacles qui s'y opposaient étaient une application trop rigide des règles en vigueur, des sanctions "laxistes" en cas de violation des DPI ainsi que le manque de ressources. Ce rapport met l'accent sur les déficiences dans l'administration du système de DPI, telles que l'absence de mécanisme efficace de coordination de ses divers éléments constitutifs, l'inadéquation des moyens de communication entre les autorités, les entreprises et les consommateurs, l'insuffisance des ressources financières et humaines consacrées à la protection de la propriété intellectuelle, l'absence de traitement systématique des problèmes de propriété des DPI et les incitations au recours à des technologies financées sur fonds publics, et il attire l'attention sur les lacunes dont souffre sous certains aspects le cadre

$30 \quad$ Article de l'Agence de presse Xinhua (“Chine nouvelle”) daté du 9 février 2006.

31 Ce plan d'action a été élaboré par le Bureau du Groupe national de travail sur la protection des DPI (National IPR Protection Working Group Office) et engage divers services et organismes gouvernementaux. Ce document était disponible sur le site internet de l'Office public de la propriété intellectuelle au 11 avril 2006, à l'adresse suivante : http://ipr2.mofcom.gov.cn/aarticle/speechactivity/200603/20060301681736.html.

32 D'après le Quotidien du Peuple en ligne (29 août 2006), en application du plan d'action pour la protection des DPI, le gouvernement a établi 50 centres permettant de signaler et de porter plainte contre les violations de la propriété intellectuelle, dans des villes réparties sur l'ensemble du territoire chinois. Une hot line nationale pour le signalement des infractions à la législation sur les DPI et le dépôt de plaintes à l'encontre de leurs auteurs a en outre été mise en place (http://english.people.com.cn/200608/29/eng20060829_297752.html). Le gouvernement a par ailleurs créé un site web bilingue (chinois/anglais) pour fournir des informations sur les droits de propriété intellectuelle en Chine (http://www.ipr.gov.cn/en/index.shtml). 
juridique applicable aux DPI. Il met en outre en évidence la nécessité de mettre en place des institutions chargées de mener la lutte antitrust et de promouvoir la concurrence afin de compléter le système de DPI. Il souligne la nécessité de respecter les normes internationales minimales en matière de DPI, dont celles instaurées par l'Accord sur les ADPIC. Les auteurs appellent à définir une stratégie nationale cohérente en matière de DPI dans le respect des limites imposées par les traités internationaux.

30. Lors d'un atelier de haut-niveau organisé en 2004 par l'OCDE et les autorités chinoises, les participants ont salué les progrès accomplis par la Chine dans l'élaboration d'un système de DPI moderne [OCDE (2005g)]. Ils n'en ont pas moins également souligné les obstacles à la mise en œuvre d'un mécanisme efficace pour assurer le respect des DPI. L'application de la législation en la matière repose sur une approche à facettes multiples associant tribunaux, fonctionnaires des douanes et autorités administratives. Un office interministériel a été établi pour coordonner l'action des 21 ministères du gouvernement associés à la mise en œuvre de la politique en matière de DPI et aux efforts pour assurer le respect de ceux-ci. Le recours à la voie administrative pour imposer le respect de la législation en la matière constitue une caractéristique fondamentale du système chinois qui permet de réprimer à peu de frais, rapidement et à l'échelon local les infractions sans qu'il soit besoin de passer par les tribunaux. Les autorités chinoises accordent une grande importance à cet outil du fait que certains secteurs de la population n'ont qu'une conscience limitée des problèmes relatifs aux DPI. (Les autorités chinoises considèrent l'éducation comme un élément clé des moyens mis en œuvre pour améliorer la protection des DPI, aussi ont-elles redoublé d'efforts en ce domaine.) Certains participants à l'atelier de l'OCDE ont toutefois souligné certains problèmes concernant le recours à la voie administrative pour assurer le respect des DPI : sanctions parfois faibles ou non transparentes en cas de violation, manque de coordination entre les organes chargés d'appliquer la réglementation, ou "protectionnisme local" et laxisme dans la mise en œuvre de la protection des DPI par les autorités locales, entre autres défaillances. Au-delà de l'approche administrative, le système judiciaire offre des voies de recours au civil et au pénal. Ces voies de recours peuvent certes déboucher sur des mesures plus dissuasives, mais elles présentent également certains inconvénients. Les procédures civiles peuvent par exemple imposer des coûts relativement élevés aux demandeurs tout en n'aboutissant qu'à de faibles sanctions pour les parties condamnées. La possibilité d'engager des poursuites pénales peut quant à elle n'être envisageable que pour les affaires dépassant des seuils élevés et demeurer insuffisamment utilisée par les autorités administratives saisies des cas d'atteinte aux DPI. Enfin, l'action des services des douanes peut il est vrai être un moyen efficace pour limiter les exportations de produits de contrefaçon, mais elle se heurte à des limites pratiques compte tenu du volume considérable des échanges de marchandises.

31. Un Livre blanc sur la protection des DPI (White Paper on IPR Protection) publié par le Bureau d'information du Conseil d'État (2005) présente une vue d'ensemble de l'évolution de la protection des DPI en Chine et offre une évaluation optimiste de l'efficacité globale du système actuellement en place, tout en reconnaissant qu' "il existe encore des violations des DPI dans certains secteurs et certains domaines en Chine, dont certaines sont très graves." De même, un rapport rédigé par des représentants internationaux de l'industrie salue les améliorations enregistrées en 2005 en matière de respect des DPI, du fait principalement de l'appui apporté par le ministère de la Sécurité publique, et juge positive la création par ce dernier, le $1^{\mathrm{er}}$ janvier 2006, d'une nouvelle unité chargée des violations de la propriété intellectuelle, dotée d'un effectif de 6 agents à plein temps [IACC (2006)]. Ce même rapport n'en estime pas moins que les efforts pour assurer le respect des DPI demeurent "nettement insuffisants". Il met en évidence de modestes augmentations du nombre d'actions à l'encontre des contrefacteurs de marques, mais souligne que presque aucune n'a été engagée contre ceux qui se rendent coupables d'atteintes aux droits d'auteurs ni contre les exportateurs de produits de contrefaçon. Il note également que la situation varie selon les régions, la province de Guangdong étant citée comme un "point noir" nécessitant des ressources supplémentaires pour lutter contre les contrefacteurs.

32. Au second semestre de 2005, le ministère japonais de l'Économie, du Commerce et de l'Industrie 
(Ministry of Economy, Trade and Industry ou METI) a mené une enquête auprès des sociétés japonaises ayant exercé des activités en Chine en 2003 et $2004 .{ }^{33}$ Les entreprises ayant répondu à l'enquête ont été au nombre de 134. Tout en saluant certains aspects positifs du système (dont les efforts déployés par les douanes chinoises pour tenter d'empêcher les exportations de produits de contrefaçon), ces entreprises mentionnaient, entre autres problèmes, les dommages subis du fait des violations de leur propriété intellectuelle, un manque de transparence sur certains points (tels que l'issue des procédures administratives engagées), des disparités régionales du point de vue des moyens de faire respecter les droits, et les difficultés à obtenir réparation par la voie judiciaire (au civil ou au pénal) et à déboucher sur une issue satisfaisante.

33. Dans un rapport de 2005 au Congrès des États-Unis, l'USTR reconnaît que "les efforts de la Chine pour mettre son cadre de lois, de réglementations et de règles de mise en œuvre en conformité avec l'Accord sur les ADPIC ont été pour une large part satisfaisants, bien que certaines améliorations demeurent nécessaires, en particulier dans les secteurs en rapide expansion, comme dans le cas de la protection des droits d'auteur sur internet." Les problèmes les plus graves se posent plutôt dans les domaines en rapport avec l'application de la législation. Le rapport de l'USTR fait part d'une communication d'une association professionnelle selon laquelle “...l'appropriation des droits de propriété intellectuelle a pris en Chine une telle ampleur qu'elle a eu un impact sur les prix internationaux, perturbé les réactions d'ajustement de l'offre [et] modifié les modèles d'activité économique [...]", entre autres effets. $^{34}$

34. L'ouvrage Études économiques de l'OCDE: Chine (2005a) récemment publié a constaté que la réforme des DPI est de plus en plus cruciale pour les innovateurs chinois, qui ont parfois du mal à obtenir que leurs DPI soient protégés au-delà de leur province d'origine. Ils se heurtent également à des difficultés du fait du manque d'objectivité des autorités judiciaires locales. Cette étude suggère que la situation pourrait être améliorée en transférant à l'administration centrale une partie des responsabilités en matière de financement des tribunaux, ainsi qu'en accroissant la spécialisation de ceux-ci pour ce qui est de la propriété intellectuelle.

\section{La Chine, les DPI et l'OMC}

35. Certains pays membres de 1'OMC ont actuellement recours à deux dispositions de 1'OMC pour chercher à obtenir davantage d'informations sur la mise en œuvre des réformes des DPI en Chine et

33 METI (2005), Field Survey for Infringement of Intellectual Property Rights in China (Final Report), disponible à l'adresse suivante : www.meti.go.jp, 23 juin 2005.

34 Les États-Unis ont exprimé des craintes au sujet des DPI au sein de la Commission conjointe sino-américaine sur le commerce et les échanges (U.S.-China Joint Commission on Commerce and Trade) et admettent que des progrès ont été accomplis en conséquence [USTR (2005)]. Par exemple, les violations des DPI sont désormais plus sévèrement sanctionnées et la Chine a lancé une campagne nationale de sensibilisation aux DPI via la radio, la télévision et la presse écrite. La Chine s'est également engagée à ratifier les traités relatifs à internet de l'Organisation mondiale de la propriété intellectuelle, et elle a interdit l'usage de logiciels piratés au sein de l'administration publique. Les "traités relatifs à internet" de l'OMPI incluent le Traité sur les droits d'auteur (1996) et le Traité sur les interprétations et les phonogrammes (1996). La ratification de ces deux traités va audelà des exigences minimales de l'Accord sur les ADPIC. Néanmoins, un examen "hors cycle" effectué par l'USTR en vertu des dispositions de l'article spécial 301 de la loi sur le commerce extérieur des États-Unis a constaté la persistance de déficiences graves dans le régime chinois de protection des DPI et a par conséquent inscrit la Chine sur la "liste des pays à surveiller" prioritairement en vertu de l'article spécial 301. Dans un récent rapport sur les relations commerciales entre les États-Unis et la Chine, l'USTR (2006) notait que "la protection des DPI est une des principales déficiences de la Chine.” 
encourager de nouveaux progrès en ce qui concerne la protection de ceux-ci. ${ }^{35}$ Ces échanges d'informations aident à mettre en lumière de manière structurée les progrès accomplis et les problèmes qui subsistent, sous la surveillance potentielle des pays membres de l'OMC. L'OMC procède en outre périodiquement à un examen de la politique commerciale des pays membres. Le premier de ces examens consacrés à la Chine a eu lieu en avril 2006. Les problèmes relatifs aux DPI y sont abordés et le rapport du Secrétariat de l'OMC tout comme la réunion d'examen offriront de nouvelles occasions de mesurer les progrès accomplis par la Chine dans la mise en œuvre de ses engagements dans le cadre de l'Accord sur les ADPIC.

36. La première des deux dispositions examinées ici est en l'occurrence l'article 18 du Protocole d'accession de la Chine, qui exige que le Conseil des ADPIC examine la mise en œuvre par la Chine de l'Accord sur les ADPIC chaque année pendant cinq ans après l'accession. Le Conseil des ADPIC remet au Conseil général de l'OMC un rapport sur les résultats de chaque examen annuel. ${ }^{36}$ En 2005, par exemple, le Japon, les Communautés européennes et les États-Unis ont eu recours à ce moyen pour formuler des questions et des observations concernant la protection des DPI par la Chine. ${ }^{37}$ Dans le cadre de ce processus, la Chine a soumis une communication décrivant brièvement les initiatives prises au cours des années précédentes dans les domaines législatif et administratif comme en matière d'application de la réglementation. En 2005, la réunion organisée au titre du mécanisme d'examen transitoire s'est tenue en octobre et le procès-verbal en a ensuite été rendu public. Y sont consignées les réponses du représentant de la Chine et l'échange de vues avec les membres qui s'en est suivi, dont une grande partie a porté sur les problèmes d'application de la réglementation.

37. La seconde disposition examinée ici est l'article 63 de l'Accord sur les ADPIC, qui traite des problèmes de transparence. Cet article exige que chacun des membres publie et notifie au Conseil des ADPIC les lois et règlements relatifs à la protection des DPI, ainsi que certaines décisions judiciaires et administratives définitives qui s'y rapportent. Les membres de l'OMC ont le droit de soumettre des questions écrites sur ces sujets. Le Japon, la Suisse et les États-Unis en ont pour la première fois fait usage en 2005. Dans les réponses apportées à ces questions, la Chine a souligné l'obligation d'interpréter et d'appliquer l'accord de bonne foi et sa volonté de renforcer la coopération avec tous les membres de l'OMC en ce qui concerne la protection des DPI. Elle a demandé des éclaircissements sur certains points pour chacune des questions et a noté que si l'article 63.3 de l'Accord sur les ADPIC mentionne le droit de demander des informations il "n'indique nullement que le pays membre à qui est adressée cette demande soit tenu d'y donner suite. Pas plus qu'il ne fixe de formalités ni de délais pour une éventuelle réponse." Dans un nouveau courrier, le Japon, la Suisse et les États-Unis ont tous trois affirmé leur volonté d'établir une coopération fructueuse avec la Chine sur ces sujets mais ont insisté sur le fait que leurs demandes d'information ne sont pas sans rapport avec les droits qui sont les leurs en vertu de l'Accord et exprimé le souhait que la Chine y réponde de bonne foi.

38. La situation actuelle concernant la protection des DPI en Chine demeure mitigée, opinion dont se sont fait l'écho diverses parties intéressées des milieux gouvernementaux, de l'industrie nationale et des

35 Il convient de noter que les pays membres de l'OCDE (tels que les États-Unis, l'UE et le Japon) sont également engagés dans une coopération technique bilatérale avec la Chine en vue de contribuer au renforcement de l'efficacité du système chinois de protection des DPI et de promouvoir la transparence et les échanges mutuels d'informations.

36. $\mathrm{OMC}(2005), \mathrm{IP} / \mathrm{C} / 39$.

37 À titre de référence, voir les documents de l'OMC suivants (2005) : IP/C/W/451 (communication du Japon), IP/C/W/450 (communication des Communautés européennes), et IP/C/W/453 et 453/Add.1 (communications des États-Unis). Le bref rapport factuel soumis par la Chine en vertu de l'article 18 [IP/C/W/460] décrit les grandes évolutions intervenues au cours de l'année précédente sur le plan législatif comme en matière d'application effective de la réglementation en vigueur. 
entreprises multinationales. La plupart des données font apparaitre de considérables progrès dans la mise en place d'un régime de DPI adapté aux besoins d'une économie de marché moderne. Subsistent toutefois des déficiences de taille - en particulier en ce qui concerne le contrôle de l'application de la réglementation - qui portent préjudice aux intérêts nationaux et internationaux. Compte tenu de l'échelle de l'économie chinoise, ces insuffisances risquent d'avoir des répercussions mondiales. Dans le même temps, un nouveau renforcement du système chinois de protection des DPI pourrait offrir d'importants avantages potentiels, dont un transfert accru de technologies (question qui pourrait faire à l'avenir l'objet de nouvelles recherches au sein de la Direction des échanges).

\section{Flux commerciaux de la Chine}

39. La Chine est un important moteur de croissance de l'économie mondiale, elle stimule aussi bien l'offre que la demande mondiales et bon nombre de ses industries sont totalement intégrées dans la chaîne d'approvisionnement mondiale. Les exportations et les importations de marchandises de la Chine ont connu une considérable expansion au cours des deux dernières décennies, et l'excédent commercial du pays s'est élevé à 102 milliards USD en 2005 (figure 1). ${ }^{38}$

Figure 1. Chine : Évolution du commerce extérieur, diverses années

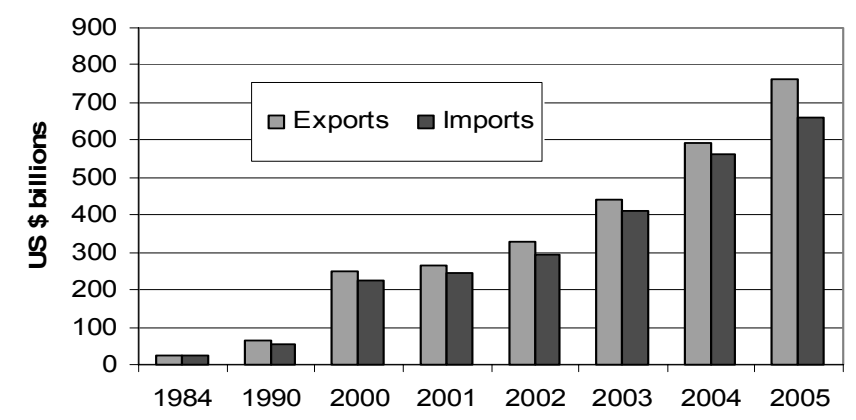

Source: Base de données statistiques de l'ONU sur le commerce de marchandises (COMTRADE); il convient de noter que les chiffres de 2005 ont été communiqués par le ministère chinois du Commerce.

40. Tout comme dans le cas des marchandises, les échanges chinois ont enregistré ces dernières années un assez considérable essor dans le domaine des services. Considéré il fut un temps comme ne pouvant faire l'objet d'échanges, ce secteur a connu au cours de la dernière décennie une importante augmentation de sa part dans l'ensemble des échanges. Les exportations de services de la Chine demeurent toutefois inférieures à celles de marchandises. En 2004, elles ne représentaient que $2.8 \%$ de l'ensemble des échanges mondiaux (tableau 4).

Tableau 4. Part des échanges de services de la Chine dans le total mondial, diverses années

(Pourcentage)

\begin{tabular}{lccc}
\hline & $\mathbf{1 9 9 4}$ & $\mathbf{2 0 0 1}$ & $\mathbf{2 0 0 4}$ \\
Exports & 1.54 & 2.18 & 2.79 \\
Imports & 1.48 & 2.53 & 3.25 \\
Total Services Trade & 1.46 & 2.47 & 3.14 \\
\hline \multicolumn{2}{l}{ Source : Statistiques de la balance des paiements du FMI (2006). }
\end{tabular}

38 Ministère du Commerce, "Imports and Exports 2005", tableau 2, 20 février 2006, http://english.mofcom.gov.cn/aarticle/statistic/hkmacaotaiwan/200602/20060201546309.html. 
41. Les exportations de marchandises chinoises comptaient pour $90 \%$ des exportations totales du pays, chiffre nettement supérieur à la moyenne mondiale, qui s'élevait à un peu plus de $80 \%$. Les exportations de services n'en représentent qu'un peu moins de 10\%, contre une moyenne mondiale de $20 \%$ (tableau 5). Cela donne à penser que les exportations de services de la Chine demeurent sous-développées et que les exportations de marchandises ont été le principal facteur d'intégration du pays dans l'économie mondiale.

Tableau 5. Échanges de biens et de services, dans le monde et en Chine

\begin{tabular}{lccccc}
\multicolumn{5}{c}{ (Pourcentage) } \\
\hline \multirow{3}{*}{ Exports } & \multicolumn{3}{c}{ Goods } & \multicolumn{2}{c}{ Services } \\
& 1994 & 80 & 86 & 20 & 14 \\
& 2001 & 80 & 89 & 20 & 11 \\
& 2004 & 80 & 90 & 20 & 10 \\
Imports & & & & & \\
& 1994 & 79 & 85 & 21 & 15 \\
& 2001 & 80 & 85 & 20 & 15 \\
& 2004 & 80 & 88 & 20 & 12 \\
\hline
\end{tabular}

Source : Statistiques de la balance des paiements du FMI (2006).

42. Contrairement à ce qui s'est produit dans le commerce de marchandises, où les exportations ont enregistré une plus forte expansion que les importations, la croissance des importations a été plus rapide que celle des exportations dans les secteurs de services de la Chine, ce qui a contribué à creuser progressivement le déficit de sa balance des services, apparu au début des années 90 . Cela suggère que le degré de spécialisation de la Chine n'a pas évolué au cours de la période analysée (en d'autres termes, le pays est demeuré importateur net de services). Le déficit croissant des échanges de services implique que la capacité d'exportation de la Chine est très limitée (et qu'elle est en outre restreinte par des obstacles élevés aux échanges de services dans les secteurs où elle possède un avantage comparatif). Cet état de choses reflète la dualité de l'économie chinoise : l'ouverture des échanges et de l'IDE dans le secteur des produits manufacturés a favorisé l'émergence d'un secteur pour une large part privé ${ }^{39}$ qui contraste avec le haut niveau de propriété publique et l'importance des obstacles réglementaires dans le secteur des services, lesquels expliquent les moins bonnes performances de celui-ci en matière d'échanges internationaux.

\section{Composition des échanges de la Chine}

43. La composition des échanges a évolué à mesure qu'ils se sont développés avec le temps. Le tableau 6 retrace la forte augmentation de la valeur des exportations de produits manufacturés et le net accroissement au fil des ans des importations de combustibles, d'énergie et de biens d'équipement.

39 Pour ce qui est des politiques sectorielles, l'accent a été mis sur l'investissement dans les activités manufacturières tournées vers l'exportation, d'où une expansion plus rapide de celles-ci. 
Tableau 6. Chine : Principaux produits dans le commerce de marchandises

\begin{tabular}{rcccc}
\multicolumn{5}{c}{ (milliards USD) } \\
\hline & $\mathbf{1 9 8 4}$ & $\mathbf{1 9 9 4}$ & $\mathbf{2 0 0 3}$ & $\mathbf{2 0 0 4}$ \\
Total Exports (fob), of which: & 26.1 & 121.0 & 438.2 & 593.4 \\
Food & 3.2 & 10.0 & 17.5 & 18.8 \\
Manufactures & 14.2 & 101.3 & 403.6 & 552.8 \\
Total Imports (cif), of which: & 27.4 & 115.6 & 412.7 & 561.4 \\
Food & 2.3 & 3.1 & 5.9 & 9.1 \\
Fuel and energy & 0.14 & 4.0 & 29.2 & 48.0 \\
Capital goods & 7.2 & 51.2 & 192.9 & 252.6 \\
\hline
\end{tabular}

Source: Banque mondiale, Base de données des indicateurs du développement dans le monde.

44. Si l'on examine de plus près la composition sectorielle des services, on constate que les services de voyages, les services aux entreprises et les services de transport ont été les principaux secteurs à avoir fait l'objet d'échanges au cours de la période 1990 - 2004 (tableau 7). Pour ce qui est des exportations, ce sont celles de services aux entreprises qui ont connu la plus forte augmentation, alors que celles de services financiers et de services d'assurance ont enregistré une baisse considérable entre 1990 et 2004. Par contre, du point de vue des importations, les services d'assurance et les services de transport paraissent être les secteurs les plus dynamiques.

Tableau 7. Chine : Composition du commerce de services

\begin{tabular}{|c|c|c|c|c|}
\hline \multicolumn{5}{|c|}{ (millions USD et \%) } \\
\hline SERVICES - Total trade & $\begin{array}{l}1990 \\
1503\end{array}$ & $\begin{array}{c}1994 \\
321\end{array}$ & $\begin{array}{c}2001 \\
-5933\end{array}$ & $\begin{array}{r}2004 \\
-9699\end{array}$ \\
\hline Services exports & 5855 & 16620 & 33334 & 62434 \\
\hline Transportation services & 46.2 & 18.5 & 13.9 & 19.3 \\
\hline Travel & 29.7 & 44.1 & 53.4 & 41.2 \\
\hline Other services & 24.1 & 37.4 & 32.7 & 39.4 \\
\hline Communications & 2.7 & 4.2 & 0.8 & 0.7 \\
\hline Construction & na & na & 2.5 & 2.4 \\
\hline Insurance & 3.9 & 10.2 & 0.7 & 0.6 \\
\hline Financial & na & na & 0.3 & 0.2 \\
\hline Computer and information & na & na & 1.4 & 2.6 \\
\hline Royalties and licence fees & na & na & 0.3 & 0.4 \\
\hline Other business services & 15.7 & 21.3 & 25.3 & 32.0 \\
\hline Personal, cultural, and recreational & na & na & 0.1 & 0.1 \\
\hline Government, n.i.e. & 1.8 & 1.6 & 1.3 & 0.6 \\
\hline Services imports & 4352 & 16299 & 39267 & 72133 \\
\hline Transportation services & 74.6 & 46.8 & 28.8 & 34.0 \\
\hline Travel & 10.8 & 18.6 & 35.4 & 26.5 \\
\hline Other services & 14.6 & 34.6 & 35.7 & 39.4 \\
\hline Communications & 0.3 & 0.9 & 0.8 & 0.7 \\
\hline Construction & na & na & 2.2 & 1.9 \\
\hline Insurance & 2.2 & 11.5 & 6.9 & 8.5 \\
\hline Financial & na & na & 0.2 & 0.2 \\
\hline Computer and information & na & na & 0.9 & 1.7 \\
\hline Royalties and licence fees & na & na & 4.9 & 6.2 \\
\hline Other business services & 6.7 & 19.0 & 19.1 & 19.3 \\
\hline Personal, cultural, and recreational & na & na & 0.1 & 0.2 \\
\hline Government, n.i.e. & 5.5 & 3.2 & 0.6 & 0.7 \\
\hline
\end{tabular}

Source : Statistiques de la balance des paiements du FMI (2006). 


\section{Section II. Impact de la Chine sur l'économie mondiale}

45. La part de la Chine dans le commerce mondial a régulièrement augmenté depuis qu'elle a commencé à réduire ses droits de douane et à ouvrir son économie aux échanges. Depuis son adhésion à l'OMC, sa part dans le commerce mondial de marchandises a presque doublé, passant de $3.7 \%$ en 2000 à $6.4 \%$ en 2005 (voir la figure 2). En 2005, la Chine est passée devant le Japon, pour devenir la troisième puissance commerciale mondiale, après l'Allemagne et les États-Unis.

Figure 2. Total des échanges mondiaux de marchandises par pays, 2000-2005

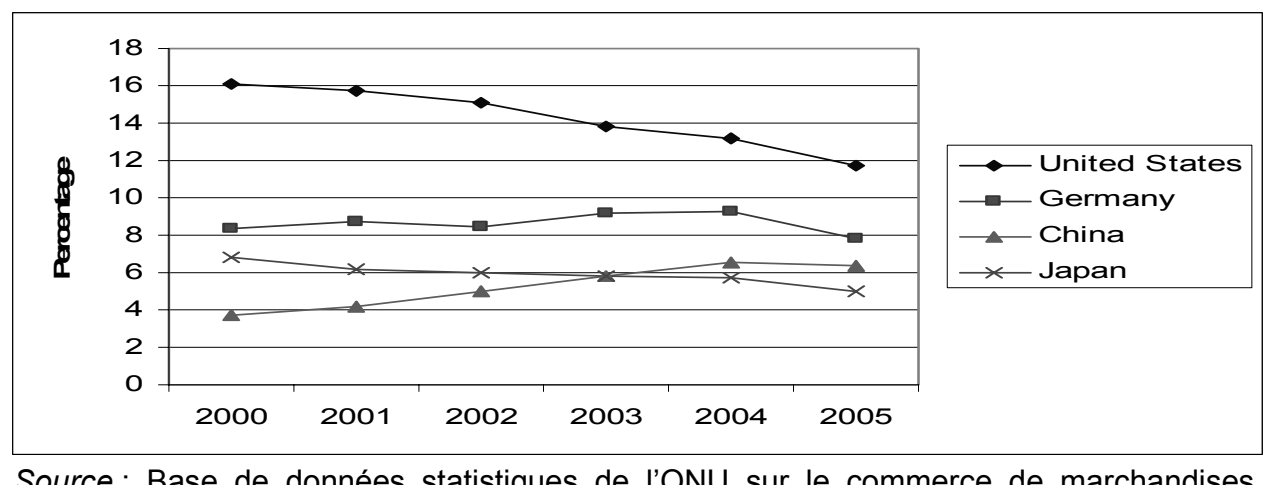
(COMTRADE), 2006

\section{Échanges de marchandises entre la Chine et les pays de l'OCDE}

46. L'interdépendance commerciale croissante entre la Chine et de nombreux pays membres de l'OCDE n'est que plus manifeste depuis son accession à l'OMC. En 2005, l'UE a été le principal partenaire commercial de la Chine, suivie de près par les États-Unis et le Japon (figure 3). Le montant total des échanges de la Chine a atteint 217 milliards USD avec l'UE-25, soit une progression de près de $23 \%$ par rapport à l'année précédente, 211 milliards USD avec les États-Unis, ce qui représente une augmentation de près de $25 \%$, et 184 milliards USD avec le Japon, chiffre de près de $10 \%$ plus élevé qu'en $2004 .{ }^{40}$ En 2005, le montant total de ses échanges avec les 10 nouveaux membres de l'UE s'est élevé à 11 milliards USD, ses principaux partenaires parmi ceux-ci étant la Pologne (3.1 milliards USD), la Hongrie ( 2.3 milliards USD), la République tchèque ( 2 milliards USD) et la République slovaque ( 0.5 milliard USD). ${ }^{41}$

\footnotetext{
40 Ministère du Commerce, "Imports and Exports 2005", tableau 2, 20 février 2006. http://english.mofcom.gov.cn/column/print.shtml?/statistic/hkmacaotaiwan/200602/20060201546309. Ibid., tableau 1.
} 
Figure 3. Principaux partenaires commerciaux de la Chine, 2005

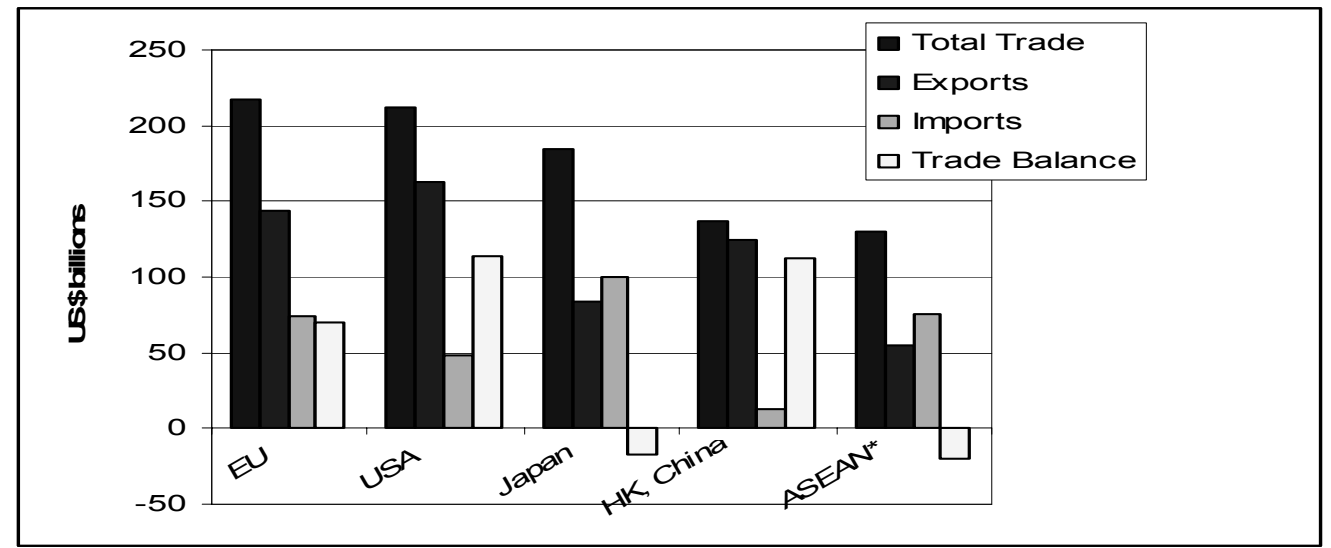

* Indonésie, Malaisie, Philippines, Singapour, Thaïlande, Brunei, Cambodge, Laos, Myanmar, et Vietnam Source : Ministère chinois du Commerce

47. Depuis la fin des années 90, la Chine et la Russie se sont attachées à mettre en place un partenariat stratégique qui prévoit le renforcement des relations commerciales bilatérales. ${ }^{42} \mathrm{La}$ coopération économique et les échanges entre la Russie et la Chine sont en plein essor et constituent désormais un important pilier et un important moteur de leurs relations bilatérales. En 2005, le volume des échanges entre la Chine et la Russie a atteint 29.1 milliards USD, soit $37.1 \%$ de plus que l'année précédente. Le Président Poutine a récemment déclaré que les deux pays se sont fixés pour objectif de développer le volume de leurs échanges bilatéraux pour le porter à 60 milliards USD d'ici $2010 .{ }^{43}$ La Chine est désormais le quatrième partenaire commercial de la Russie, alors que la Russie est le huitième partenaire commercial de la Chine. Les exportations de la Russie vers la Chine sont principalement dominées par l'énergie et les matières premières alors que la Chine vend à la Russie des produits de plus haute technologie.

48. La Chine est devenue l'un des principaux partenaires commerciaux d'une majorité de pays de l'OCDE. Grâce aux efforts qu'elle a déployés depuis le début des années 90 pour mettre en place un régime commercial plus ouvert, sa part des marchés d'exportation de l'OCDE s'est spectaculairement accrue (tableau 8), surtout au Japon, aux États-Unis, en Corée, en Australie, au sein de l'UE-15 et en Nouvelle-Zélande. La Chine est en passe de devenir à bref délai un moteur de croissance régional en Asie, compte tenu que ses importations proviennent en proportions croissantes de la région. On pourrait faire valoir que sa croissance économique est de plus en plus tributaire de la région et que cela explique l'intérêt stratégique que revêt pour le pays l'approfondissement des relations commerciales en Asie. Elle continue à développer les chaînes de production régionales avec le concours des économies et des marchés asiatiques, aussi s'efforce-t-elle activement de conclure des accords commerciaux préférentiels avec ses voisins. Elle est en outre en train de négocier des accords bilatéraux de libre-échange avec l'Australie et la NouvelleZélande et a engagé des pourparlers avec l'Inde, la Corée et le Japon. Il importe de noter que la Chine a

42 En 2001, le Président chinois de l'époque, Jiang Zemin, et le Président russe, Vladimir Poutin, ont signé le Traité sino-russe de bon voisinage, d'amitié et de coopération. En mai 2003, le Président chinois Hu Jintao et Vladimir Poutin ont signé une déclaration commune à Moscou, inaugurant une nouvelle ère de développement du partenariat stratégique sino-russe. En mars 2006, le ministère chinois du Commerce et le ministère russe du Développement économique et du Commerce organiseront conjointement le Forum économique sino-russe dans le cadre de 1"“Année de la Russie" en Chine.

43 Voir S. Blagov (2006) "Russia registers growth in trade with China, but increase due to higher prices, not more turnover", Eurasia Daily Monitor, 7 février 2006. http://jamestown.org/edm/article.php?article_id=2370750. 
tout mis en œuvre pour parvenir à la signature avec l'ANASE d'un accord régional de libre-échange couvrant plus d'une dizaine de pays du Sud-Est asiatique dès $2015 .^{44}$

\section{Tableau 8. Part de la Chine sur les principaux marchés}

\begin{tabular}{lcccccc}
\multicolumn{7}{c}{ (Pourcentage des importations totales) } \\
\hline Partner & $\mathbf{1 9 9 0}$ & $\mathbf{2 0 0 0}$ & $\mathbf{2 0 0 1}$ & $\mathbf{2 0 0 2}$ & $\mathbf{2 0 0 3}$ & $\mathbf{2 0 0 4}$ \\
Japan & 5.2 & 14.5 & 16.6 & 18.3 & 19.7 & 20.8 \\
US & 3.1 & 8.6 & 9.3 & 11.1 & 12.5 & 13.8 \\
Korea & 2.1 & 8.1 & 9.5 & 11.6 & 12.4 & 13.4 \\
Australia & 2.7 & 7.9 & 9.0 & 10.3 & 11.3 & 13.0 \\
EU 15 & 2.5 & 6.2 & 6.8 & 7.7 & 9.1 & 10.7 \\
New Zealand & 1.2 & 6.3 & 7.0 & 8.0 & 9.0 & 10.2 \\
Canada & 1.0 & 3.2 & 3.7 & 4.6 & 5.5 & 6.8 \\
Russia* & 1.6 & 2.1 & 3.9 & 5.7 & 5.7 & 6.3 \\
Mexico & 0.8 & 1.7 & 2.4 & 3.7 & 5.5 & na \\
Turkey & 1.1 & 2.4 & 2.3 & 2.7 & 3.9 & 4.8 \\
\hline
\end{tabular}

* Le chiffre indiqué pour 1990 correspond de fait à 1996.

Source: Base de données statistiques de l'ONU sur le commerce de marchandises (COMTRADE) ; données de l'UE établies à partir des Statistiques du commerce international de l'OCDE.

\section{Échanges de services entre la Chine et les pays de l’OCDE}

49. On commence certes à disposer d'informations sur les échanges de services par pays partenaire dans les pays de l'OCDE, mais rares sont les pays en développement qui communiquent ce type de données. En vue d'identifier les principales caractéristiques des échanges de services de la Chine avec les pays de l'OCDE, des estimations ont été établies à partir de la base de données statistiques de l'OCDE sur les échanges internationaux de services par pays partenaire ainsi que des données sur les exportations et les importations communiquées par la Chine au FMI. Bien qu'il faille garder à l'esprit que ces estimations présentent un haut degré d'incertitude, les données disponibles montrent que $72 \%$ des exportations totales de services de la Chine avaient pour destination les pays de l'OCDE en 1999. Ce pourcentage a diminué au fil du temps pour tomber à 50\% en 2003. On se serait attendu à ce que les exportations de la Chine vers les pays de l'OCDE augmentent à mesure que la qualité de ses services s'accroît. Les données suggèrent plutôt que, pour ce qui est des exportations de services de la Chine, les pays non membres de l'OCDE sont en train de devenir des partenaires commerciaux plus importants.

50. Dans les échanges de services la situation est toute autre que dans les échanges de marchandises, où la Chine est devenue un important partenaire commercial pour la majorité des économies de l'OCDE. La part de la Chine dans les exportations et les importations de services des pays de l'OCDE demeure dans la plupart des cas extrêmement faible (insignifiante), sauf pour ce qui est de ses voisins asiatiques (tableaux 9 et 10).

44 Dont Brunei, l'Indonésie, la Malaisie, les Philippines, Singapour, la Thaïlande, le Vietnam, le Laos, le Myanmar et le Cambodge. Après la création de la zone de libre-échange avec l'ANASE, le commerce intrarégional devrait, espère-t-on, se développer sensiblement, le volume total des échanges s'élevant à plus de 1 billion USD. Certains pays de la région considèrent toutefois que l'expansion de la Chine dans certains secteurs tels que ceux de l'électronique, de l'ameublement et des motocycles a pour effet de les évincer de leurs marchés et a donc une incidence négative sur leur économie. Voir E. Economy (2005), op. cit., p. 414. 
Tableau 9. Les 10 principales destinations des exportations de services de la Chine

(En pourcentage des importations totales de services des divers pays)

\begin{tabular}{lcccc}
\hline & $\mathbf{2 0 0 0}$ & $\mathbf{2 0 0 1}$ & $\mathbf{2 0 0 2}$ & $\mathbf{2 0 0 3}$ \\
Hong Kong, China & 30.86 & 30.71 & 30.06 & 28.56 \\
Korea & 6.91 & 7.39 & 9.61 & 9.32 \\
Japan & 3.58 & 3.65 & 4.00 & 4.34 \\
Russian Federation & na & na & na & 2.91 \\
Australia & 2.24 & 2.24 & 2.66 & 2.57 \\
Denmark & 0.33 & 2.03 & 2.33 & 2.41 \\
Finland & 0.21 & 0.21 & 0.27 & 0.97 \\
Canada & 0.68 & 0.81 & 1.26 & 0.81 \\
Italy & 0.92 & 0.89 & 1.46 & 0.68 \\
Spain & 0.58 & 0.60 & 0.64 & 0.66 \\
Source : Base de données statistiques de l'OCDE sur les échanges internationaux \\
\multicolumn{4}{c}{}
\end{tabular}

Tableau 10. Les 10 principaux pays d'origine des importations de service de la Chine

(En pourcentage des exportations totales de services des divers pays)

\begin{tabular}{lcccc}
\hline & $\mathbf{2 0 0 0}$ & $\mathbf{2 0 0 1}$ & $\mathbf{2 0 0 2}$ & $\mathbf{2 0 0 3}$ \\
\hline Hong Kong, China & 21.85 & 23.84 & 27.18 & 28.22 \\
Korea (Republic of) & 6.38 & 7.41 & 8.86 & 11.04 \\
Japan & 3.40 & 3.63 & 4.18 & 5.34 \\
Finland & 0.11 & 0.14 & 0.59 & 3.34 \\
Australia & 2.38 & 2.78 & 3.00 & 3.13 \\
Slovak Republic & na & 0.04 & na & 2.92 \\
Denmark & 2.22 & 2.69 & 2.72 & 2.82 \\
Russian Federation & na & na & na & 2.38 \\
Germany & 0.82 & 1.07 & 1.33 & 1.36 \\
\hline France & 0.49 & 0.69 & 0.86 & 1.34
\end{tabular}

Source : Base de données statistiques de l'OCDE sur les échanges internationaux de services par pays partenaire (2006).

51. L'attrait exercé par la Chine sur l'IDE a été à la mesure de l'expansion de ses échanges au cours des 25 dernières années. La prochaine section examinera les problèmes relatifs aux échanges et à l'investissement.

\section{Échanges et investissement}

52. La libéralisation des échanges et de l'investissement menée en Chine a créé un environnement attractif pour les entreprises et a par conséquent eu un important impact sur les entrées d'IDE. ${ }^{45}$ Quasiment nul en 1979, l'IDE est passé à 636 millions USD en 1983, puis à 60.6 milliards USD en $2004 .^{46}$ Le ministère du Commerce a indiqué que l'IDE a légèrement diminué pour tomber à 60.3 milliards USD en 2005. La Chine se classe depuis 1993 au premier rang des pays en développement destinataires d'IDE et elle a occupé en 2002 la première place mondiale du point de vue des entrées d'IDE. La Chine est

45 Pour une analyse détaillée de la politique de la Chine dans le domaine de l'investissement, voir OCDE (2003) Chine : progrès et enjeux de la réforme, OCDE, Paris, 2003 et OCDE (2006a) Chine: Politiques ouvertes envers les fusions et acquisitions, OCDE, Paris, 2006.

46 OCDE (2005b), Perspectives de l'investissement international, OCDE, Paris, p. 27 et CNUCED (2005a) Rapport sur l'investissement dans le monde 2005 : Les sociétés transnationales et l'internationalisation de la recherche-développement, Genève, p. xix. 
actuellement le troisième plus grand pays de destination des flux d'IDE, après les États-Unis et le Royaume-Uni (CNUCED, 2005a).

53. Plusieurs facteurs ont contribué à accroître les entrées d'IDE. La Chine a libéralisé son régime d'IDE parallèlement à la mise en œuvre de ses politiques de réforme économique et de portes ouvertes à la fin des années 70 et plus particulièrement depuis le début des années 90 . Grâce à l'adoption d'une série de lois et de règlements concernant l'IDE, la Chine a sensiblement réduit les obstacles à l'investissement et amélioré son environnement en matière d'investissement en ouvrant davantage de régions et de secteurs économiques aux investisseurs étrangers. La Chine est en outre le pays le plus peuplé au monde et dispose en conséquence d'une abondante main-d'œuvre bon marché et d'un immense marché potentiel. Conscients de ces atouts dès le début des années 80 , les pays de la région tels que le Japon et les NEI asiatiques sont devenus d'importantes sources de capitaux pour la Chine. En 2005, les entrées d'IDE en Chine provenaient de Hong Kong, Chine ${ }^{47}$ (29.8\%), du Japon (10.8\%), de l'UE (8.6\%), des États-Unis (5.1\%) et du Taipei chinois $(3.6 \%){ }^{48}$

54. Il importe toutefois de noter que les performances de la Chine dans le domaine de l'IDE doivent être resituées dans une perspective internationale. Du point de vue des entrées d'IDE par habitant, la Chine occupe une moins bonne position que tous les pays de l'OCDE sauf un, et elle se trouve même relativement mal classée parmi les pays en développement. ${ }^{49}$ En outre, le bond qualitatif n'a pas vraiment été à la mesure du bond quantitatif. L'IDE dont bénéficie la Chine se compose pour une large part d'investissements à court terme dans les industries manufacturières à forte intensité de main-d'œuvre, alors que les investissements étrangers demeurent à la traîne dans les secteurs de haute technologie et dans ceux de services. La Chine encourage actuellement l'IDE dans les produits manufacturés de haute technologie en tant que moyen d'encourager les entreprises nationales à s'élever dans la chaîne de la valeur ajoutée. Du fait de son accession à l'OMC, elle est également en train d'éliminer les obstacles en vigueur dans ces secteurs. Pour continuer à attirer l'IDE, et en particulier l'IDE de haute qualité en provenance des pays de l'OCDE, la Chine doit pleinement mettre en œuvre les engagements pris à l'OMC et procéder à des réformes complémentaires au sein du pays. Il s'agit de continuer à réduire les obstacles aux échanges et à l'investissement et de poursuivre la libéralisation des marchés intérieurs, la réforme des entreprises d'État, la mise en place d'un cadre réglementaire fondé sur l'application de règles claires et transparentes, ainsi que le renforcement de l'État de droit, en particulier dans des domaines tels que la protection des droits de propriété intellectuelle.

55. Au cours des six dernières années, le gouvernement chinois a déployé des efforts concertés pour accroître ses investissements à l'étranger. L'augmentation des investissements directs à l'étranger constitue depuis mars 2000 un objectif déclaré du gouvernement chinois. Plusieurs facteurs favorisent la politique d'investissement direct à l'étranger ou de "mondialisation". ${ }^{50}$ Premièrement, la demande croissante de ressources naturelles de la Chine a été un des principaux moteurs de l'investissement direct à l'étranger. ${ }^{51}$

47 Les investissements à l'étranger de Hong Kong, Chine, ont atteint 39.7 milliards USD en 2004. D'après l'OCDE, bien qu'il ne fasse guère de doute que les investisseurs basés à Hong Kong, Chine, comptent pour beaucoup dans l'investissement direct sur le continent, il serait trop simpliste d'attribuer le boom de l'IDE chinois au "circuit en boucle" des investissements. OCDE (2005b), Ibid., p. 27.

OCDE (2006a), op. cit., annexe statistique, tableau 5.

50 Voir CNUCED (2003), "China: an emerging FDI outward investor", E-Brève, CNUCED, Genève, 4 décembre 2003.

51 En 2005, la China National Offshore Oil Company (CNOOC), société pétrolière chinoise dont le capital est intégralement détenu par l'État, a fait une offre d'achat d'une société pétrolière américaine : UNOCAL. Elle a toutefois retiré son offre devant la forte opposition de nombreux membres du Congrès des États-Unis. 
Le gouvernement chinois a déployé des efforts concertés pour s'assurer des sources futures d'approvisionnement en pétrole, gaz naturel, minerais de fer, aluminium, bois d'œuvre et autres produits de base en Australie, en Russie et dans les pays en développement d'Afrique et d'Amérique latine. D'après les statistiques du ministère chinois du Commerce, les industries minières et extractives comptaient en 2004 pour $32.7 \%$ de l'ensemble des investissements de la Chine à l'étranger. ${ }^{52}$

56. En second lieu, les sociétés chinoises s'attachent à obtenir un transfert international de technologies et de compétences, les efforts dans ce sens revêtant notamment la forme de la création de centres de recherche-développement (R-D) dans les pays développés. Dans le $11^{\text {ème }}$ Plan quinquennal de la Chine pour 2006-2010, le gouvernement pousse les grandes sociétés à créer des établissements de R-D et invite les entreprises à prendre part aux activités de R-D de l'État. ${ }^{53}$ Les sociétés chinoises PetroChina et China Petroleum \& Chemical figurent sur la liste des 20 sociétés des pays en développement dont les dépenses de R-D sont les plus élevées (CNUCED, 2005a). Sur les 77 unités de R-D que comptait la Chine à la fin de 2004, 37 opéraient à l'étranger. Parmi celles-ci, 26 étaient localisées dans des pays développés, principalement aux États-Unis (11) et en Europe (11), les autres étant situées dans des pays en développement. ${ }^{54}$

57. Un troisième moteur de l'investissement à l'étranger tient au désir des sociétés exportatrices chinoises de soutenir leurs exportations, d'approvisionner leurs marchés et de développer leur présence sur les marchés étrangers. Les fabricants chinois de téléviseurs et d'appareils ménagers dont les capacités de production sont excédentaires sont à la recherche de possibilités de développement à l'étranger. En 2003, TCL, principal fabricant chinois de produits électroniques multimédias destinés au grand public, et la société française Thomson, leader mondial des technologies vidéo, on créé une coentreprise. En 2005, Leonovo - une des plus grandes sociétés chinoises - a acheté la division informatique personnelle d'IBM.

58. Malgré ces moteurs potentiels, les investissements directs de la Chine à l'étranger demeurent relativement modestes. D'après les estimations de la CNUCED, les flux d'investissement de la Chine à l'étranger se sont en moyenne élevés à 3 milliards USD par an sur la période 2000-2003. ${ }^{55}$ En 2004, ses investissements directs se sont élevés à 1.8 milliard USD ${ }^{56}$ - soit seulement $0.2 \%$ du total mondial. Il n'est certes pas exclu que ces chiffres soient sous-estimés, mais le gouvernement chinois considère manifestement que les entreprises chinoises ont de très bonnes chances de pouvoir développer sensiblement leurs investissements à l'étranger. En 2004, le gouvernement chinois a annoncé l'adoption de nouvelles politiques en la matière et affirmé son soutien aux entreprises chinoises qui investissent à l'extérieur. Un document publié conjointement par la Commission nationale du développement et de la réforme et la Banque d'import-export de la Chine décrit dans leurs grandes lignes les politiques d'octroi de crédits publics aux projets essentiels qui bénéficient d'un soutien de l'État. ${ }^{57}$

59. Pour comprendre la rapide expansion des échanges extérieurs de la Chine, il importe de bien mesurer à quel point les entreprises à participation étrangère ont joué un rôle crucial dans le développement de ses exportations. Il convient de garder à l'esprit que l'ouverture de la Chine aux

522004 年度中国对外直接投资统计公报（非金融部分）2004 Statistical Bulletin of China's Outward Foreign Direct Investment (Non-Finance Part), Ministère du Commerce et Bureau national des statistiques.

53 Voir Communiqué de la $5^{\text {ème }}$ session plénière du $16^{\text {ème }}$ Comité central du Parti communiste chinois, 11 octobre 2005.

54 CNUCED (2005a) op. cit., p. 150.

55 CNUCED (2004), Rapport sur l'investissement dans le monde 2004 : La montée en puissance du secteur des services, CNUCED, Genève, 2004, p. 24.

56 OCDE (2005b), op. cit., p. 34.

57 Y. Zhang (2005), China Goes Global, Foreign Policy Centre, Londres, avril 2005, pp. 13-14. 
investissements étrangers a coïncidé avec son ouverture aux échanges extérieurs. La capacité d'exportation aurait toutefois pu se développer même en l'absence de vastes entrées d'IDE, quoique sans doute à un rythme moins rapide. Les entreprises à participation étrangère comptent néanmoins pour une grande part des exportations et des importations de la Chine. Les importations des entreprises à participation étrangère ont atteint 388 milliards USD en 2005, ce qui représente 58.8\% de l'ensemble des importations. Leurs exportations se sont développées pour atteindre 444 milliards USD, soit $58.2 \%$ des exportations de la Chine en $2005 .^{58}$ La part des exportations des entreprises à participation étrangère est sensiblement plus élevée que dans les autres NEI d'Asie à un stade similaire de développement. Les entreprises à participation étrangère comptaient au milieu des années 70 pour $20 \%$ des exportations de produits manufacturés du Taipei chinois et pour $25 \%$ de celles de la Corée sur la période 1974-1978. La prépondérance des entreprises à participation étrangère est encore plus nette si seules sont prises en considération les exportations chinoises de biens industriels dans les secteurs de pointe, puisque leur part y est de 70 à $90 \%{ }^{59}$ Cette très large prépondérance des entreprises à participation étrangère au sein de l'économie chinoise témoigne également du rôle croissant joué par cette dernière dans les activités internationales de transformation. Ce point sera abordé dans la prochaine section.

\section{Rôle de la Chine dans les activités internationales de transformation}

60. Les activités internationales de transformation ont été le moteur de la phénoménale croissance des échanges extérieurs de la Chine, moteur qui a été alimenté par la libéralisation des échanges et de l'investissement menée par le pays. L'interdépendance croissante des échanges mondiaux et régionaux a généré une nouvelle configuration des échanges d'une plus grande complexité. Les réseaux de production transfrontières, qui sont à l'origine d'une internationalisation progressive du processus de production de nombreux biens et services, en offrent une illustration. Au lieu de s'attacher à développer des compétences leur permettant d'assurer la production de bout en bout des produits, les entreprises chinoises concentrent leurs efforts sur une fraction donnée du processus de production dans laquelle elles possèdent un avantage comparatif. La Chine continue de jouir d'un avantage comparatif dans le domaine des produits à forte intensité de main-d'œuvre. La libéralisation des échanges lui a permis de prendre davantage part aux réseaux internationaux de production et de pousser plus loin l'intégration avec ses partenaires commerciaux, surtout en Asie. Elle l'a également aidée à affronter la concurrence sur les marchés mondiaux en favorisant le développement de son industrie manufacturière et de ses secteurs de services. Les réseaux de production associant les économies développées et les économies émergentes ont beaucoup accru les possibilités de spécialisation à l'origine de gains d'efficacité et de réductions des coûts, tout comme celles de procéder à des échanges source d'un surcroît de bien-être. ${ }^{60}$

61. Gaulier et al. (2005) affirment que les activités internationales de transformation constituent le plus important facteur de croissance des exportations et des importations de la Chine, dont les entreprises à capitaux étrangers ont été les principaux acteurs. Ils ont constaté qu'au lieu d'exporter des produits finis vers les marchés des États-Unis et des pays européens, les entreprises exportatrices des économies développées d'Asie utilisent la Chine comme base d'exportation. Elles exportent des biens intermédiaires vers leurs filiales en Chine, où ils sont assemblés ou transformés puis réexportés, principalement vers l'UE et les États-Unis, d'où une structure triangulaire des échanges. ${ }^{61}$ Les exportations de la Chine vers l'UE et

\footnotetext{
58 Voir OCDE (2006a) Chine : Politiques ouvertes envers les fusions et acquisitions, op. cit., annexe, tableau 9.

59 G. J. Gilboy (2004), “The Myth behind China's Miracle”, Foreign Affairs, juillet/août 2004. Cet article se penche plus en détail sur la stratégie industrielle culturellement dominante en Chine.

60 Voir S. Arndt (2004), Trade Integration and Production Networks in Asia: The Role of China, Claremont McKenna College, juin 2004.

61 G. Gaulier, F. Lemoine, et D. Unal-Kesenci (2005), China's Integration in East Asia: Production Sharing, FDI \& High-Tech Trade, Centre d'études prospectives et d'informations internationales (CEPII), 9 juin 2005.
} 
les États-Unis ont en conséquence beaucoup augmenté et évincé celles du Japon et des NEI asiatiques. D'après le ministère chinois du Commerce, la part des importations destinées à la transformation dans l'ensemble des importations s'est élevée à $41.5 \%$ en 2005. Dans le même temps, les importations chinoises destinées à la transformation représenteraient d'après les estimations $36 \%$ de l'ensemble des exportations du pays. ${ }^{62}$

62. La croissance des exportations et des importations de la Chine et la composition de ses échanges font très clairement apparaître l'ampleur des échanges intra-branche au sein du pays. La figure 4 montre que les profils de croissance des exportations et des importations au cours des dernières années présentent une grande similarité. Un autre indice de la prépondérance des activités internationales de transformation peut être observé aux tableaux 11 et 12 , qui recensent les principales exportations et les principales importations de la Chine en 2004 : le pays exporte et importe des produits similaires. Les principales exportations de la Chine comprennent les machines électriques et le matériel de transport, les équipements de production d'électricité, les instruments d'optique et le matériel médical, ainsi que l'habillement et la sidérurgie. Les plus fortes variations ont lieu dans la sidérurgie, les instruments d'optique et le matériel médical, les machines et équipements électriques et les équipements de production d'électricité. Les principales importations sont constituées de machines et de matériel de transport, d'équipements de production d'électricité, d'instruments d'optique et de matériel médical, ainsi que de combustibles minéraux et de pétrole, de produits sidérurgiques, de matières plastiques et de produits chimiques.

Figure 4. Croissance des exportations et des importations

(Pourcentage)

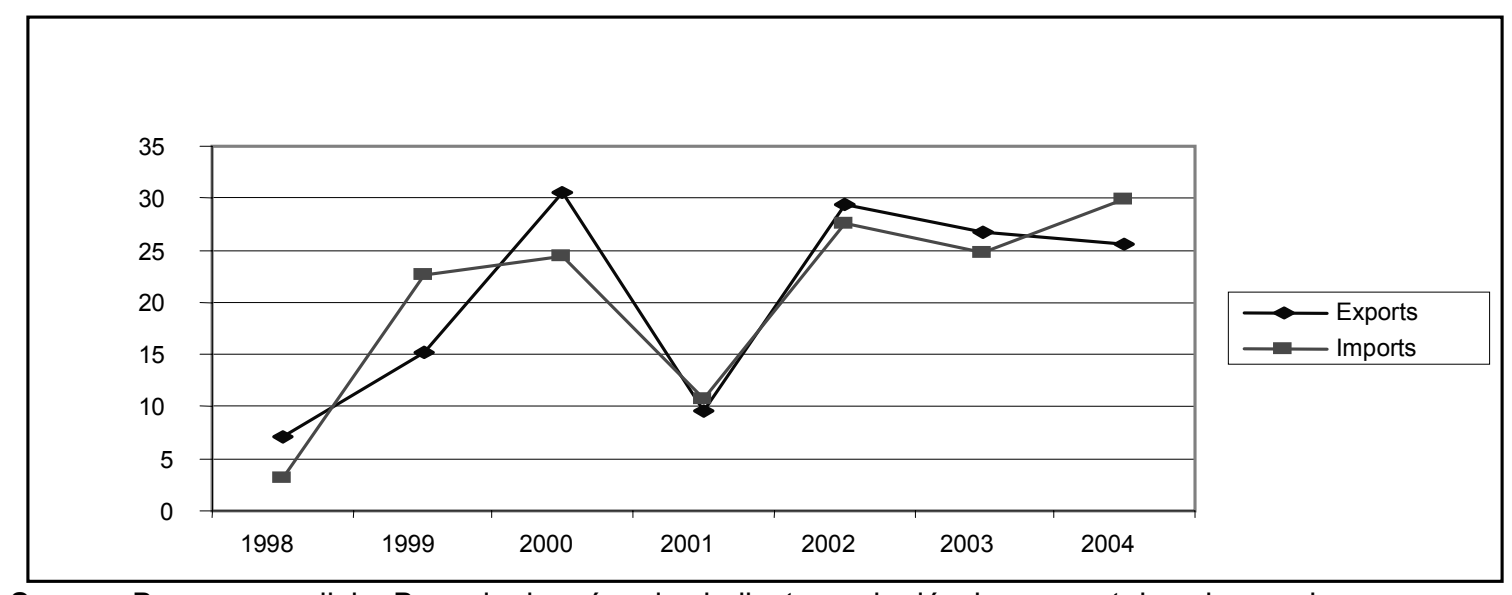

Source : Banque mondiale, Base de données des indicateurs du développement dans le monde

62 Voir ministère du Commerce, http://gcs.mofcom.gov.cn/aarticle/Nocategory/200602/20060201484560.html et http://gcs.mofcom.gov.cn/aarticle/Nocategory/200602/20060201484556.html. 
TD/TC/WP(2006)10/FINAL

Tableau 11. Principales exportations de la Chine

(milliards USD)

\begin{tabular}{lrrc}
\hline \multicolumn{1}{c}{ Commodity } & $\mathbf{2 0 0 3}$ & $\mathbf{2 0 0 4}$ & \% change \\
\hline $\begin{array}{l}\text { Electrical machinery \& } \\
\text { equipment }\end{array}$ & 88.97 & 129.66 & 45.8 \\
$\begin{array}{l}\text { Power generation } \\
\text { equipment }\end{array}$ & 83.47 & 118.15 & 41.7 \\
$\begin{array}{l}\text { Apparel } \\
\text { Iron and steel }\end{array}$ & 45.76 & 54.78 & 19.7 \\
$\begin{array}{l}\text { Furniture and bedding } \\
\text { Optics \& medical }\end{array}$ & 12.86 & 25.22 & 96.0 \\
equipment & 10.56 & 17.32 & 29.1 \\
$\begin{array}{l}\text { Footwear and parts } \\
\text { thereof }\end{array}$ & 12.96 & 15.20 & 53.6 \\
$\begin{array}{l}\text { Toys and games } \\
\text { Mineral fuel and oil }\end{array}$ & 13.28 & 15.10 & 17.4 \\
$\begin{array}{l}\text { Inorganic and organic } \\
\text { chemicals }\end{array}$ & 10.73 & 13.94 & 13.6 \\
\hline
\end{tabular}

Tableau 12. Principales importations de la Chine

(milliards USD)

\begin{tabular}{lrrr}
\hline \multicolumn{1}{c}{ Commodity } & $\mathbf{2 0 0 3}$ & $\mathbf{2 0 0 4}$ & \% change \\
\hline $\begin{array}{l}\text { Electrical machinery \& } \\
\text { equipment }\end{array}$ & 103.93 & 142.10 & 36.7 \\
$\begin{array}{l}\text { Power generation } \\
\text { equipment }\end{array}$ & 71.50 & 91.63 & 28.2 \\
$\begin{array}{l}\text { Mineral fuel and oil } \\
\text { Optics \&medical }\end{array}$ & 29.27 & 48.03 & 64.2 \\
equipment & 25.14 & 40.15 & 59.8 \\
$\begin{array}{l}\text { Iron and steel } \\
\text { Plastics and articles }\end{array}$ & 25.60 & 28.39 & 10.9 \\
thereof & 21.03 & 28.06 & 33.4 \\
$\begin{array}{l}\text { Inorganic and organic } \\
\text { chemicals }\end{array}$ & 18.74 & 27.81 & 48.4 \\
$\begin{array}{l}\text { Ore, slag and ash } \\
\text { Vehicle -other than rail }\end{array}$ & 7.17 & 17.29 & 141.0 \\
Copper & 7.17 & 13.10 & 11.2 \\
\hline
\end{tabular}

Source : Statistiques douanières de la Chine.

63. La Chine s'est imposée comme un acteur majeur sur les marchés internationaux, aussi les pays membres de l'OCDE s'intéressent-ils de plus en plus à son impact potentiel sur les marchés mondiaux et sur leurs soldes commerciaux bilatéraux. Vu l'importance que revêtent en Chine les échanges liés aux activités internationales de transformation, les soldes commerciaux bilatéraux du pays risquent de donner une image faussée de la réalité. Arndt (2004) fait valoir que les procédures habituellement utilisées pour établir la balance des paiements risquent d'induire en erreur en cas de partage de la production à l'échelle internationale. Gaulier et al. (2005) avancent l'idée que les activités de transformation sont responsables de la plus grande partie de l'excédent commercial de la Chine. Si l'on fait abstraction des flux commerciaux liés aux activités de transformation, les échanges de la Chine sont déficitaires avec l'UE alors qu'ils sont presque équilibrés avec les États-Unis. La même étude soutient que les échanges entre la Chine d'une part et l'UE et les États-Unis d'autre part présentent par essence une certaine asymétrie du fait de l'intégration de la Chine dans les réseaux de production asiatiques. Les déficits commerciaux des États-Unis et de l'UE sont liés aux activités des entreprises à participation étrangère - c'est-à-dire bien souvent leurs propres sociétés multinationales - qui tirent de vastes profits et jouissent d'une forte compétitivité grâce aux bas coûts de production dont ils bénéficient en Chine. ${ }^{63}$

64. Les échanges liés aux activités de transformation représentent une part si importante des flux commerciaux de la Chine qu'il est essentiel d'examiner tant les variations brutes que les variations nettes du volume des exportations et des importations en vue d'identifier le rôle et l'ampleur de la valeur ajoutée étrangère importée. Arndt soutient qu'il n'en est que plus crucial de faire la distinction entre importations et valeur ajoutée importée d'une part et exportations et valeur ajoutée exportée d'autre part. Certains indices montrent que la valeur ajoutée à l'intérieur du pays forme une plus grande part des échanges liés aux activités de transformation de la Chine, surtout dans le secteur de l'électronique. Près de $20 \%$ des importations de la Chine sont constituées de machines électriques et de semiconducteurs, alors que près de $15 \%$ de ses exportations consistent en matériel informatique, $11.5 \%$ en équipements de télécommunications et $10 \%$ en machines électriques et semiconducteurs. ${ }^{64} \mathrm{La}$ Direction de la science, de la technologie et de l'industrie (DSTI) de l'OCDE travaille actuellement à un projet sur la mondialisation qui

\footnotetext{
63 G. Gaulier, et al. op. cit. p.19.

64 OCDE (2005a) op. cit., p. 9.
} 
donnera lieu à une analyse des interactions mondiales à l'aide de tableaux d'entrées-sorties dans l'espoir de pouvoir déterminer la part de la valeur ajoutée dans les exportations de la Chine. ${ }^{65}$ Des données sur la situation de la Chine en 1997 et 2000 ont été réunies dans le cadre du projet et il sera procédé à un examen des évolutions intervenues au cours de cette période et au-delà.

65. Certaines données disponibles suggèrent que les activités de transformation de la Chine ont évolué au fil des ans : d'abord centrées sur les produits à forte intensité de main-d'œuvre et de basse technologie, elles se sont ensuite étendues à des produits de plus haute technologie. La composition des échanges de la Chine avec les pays qui constituent ses principaux marchés d'exportation confortent cette idée. S'agissant du Japon, de sensibles augmentations ont été observées en 2004 par rapport aux chiffres de 2003 pour ce qui est des produits finis tels que les ordinateurs personnels, les imprimantes et les autres équipements de bureau (31.2\%), les lecteurs de DVD et les autres équipements audiovisuels (32.8\%), et les téléphones portables et les autres équipements de télécommunications (58.2\%). Les semiconducteurs et autres composants électroniques ont également enregistré de fortes progressions $(82.1 \%){ }^{66}$ Les statistiques du ministère du Commerce des États-Unis montrent que la valeur des exportations chinoises de matériel informatique à destination des États-Unis sont par exemple passées de 8.1 milliards USD en 2001 à 29.5 milliards USD en $2004 .{ }^{67} \mathrm{Il}$ en est allé de même des équipements audio et vidéo, des équipements de communication, des semi-conducteurs, ainsi que des autres composants électroniques.

66. Le rapport 2004 de la Commission européenne sur la compétitivité européenne s'est penché sur les exportations de la Chine vers l'UE-15 et fait état d'une sensible amélioration de la qualité de l'apport de main-d'œuvre. Ce rapport confirme que la composition des exportations chinoises s'est modifiée durant la période allant de 1995 à 2002 : d'abord constituées de produits à forte intensité de main-d'œuvre peu qualifiée, elles font désormais une place croissante à des produits à plus forte intensité de capital humain et de savoir-faire. Certains secteurs technologiques ont connu une croissance remarquable, tels que ceux des équipements informatiques et de télécommunications, ou encore ceux des produits scientifiques, des équipements de mesure et du matériel de transport. ${ }^{68}$ Les exportations de la Chine vers l'UE-15 dans les industries technologiques sont passées de moins de $20 \%$ en 1995 à $30 \%$ en 2002 , ce qui constitue la plus grande part des exportations chinoises. Les produits à forte intensité de main-d'œuvre sont tombés de $25 \%$ à moins de $22 \%$. Bien que la Chine soit perçue comme un pays disposant d'une abondante main-d'œuvre bon marché, ces chiffres montrent qu'elle ne se contente pas d'être un fournisseur de biens industriels produits par une main-d'œuvre peu onéreuse et peu qualifiée mais qu'elle est aussi en train de devenir compétitive dans le secteur des produits à forte intensité de technologie et de savoir.

67. La part croissante de la Chine dans les exportations de biens industriels et de haute technologie à destination de bon nombre de pays de l'OCDE mérite toutefois davantage de précisions. Les importations chinoises de biens intermédiaires ont constitué un important vecteur de transfert de technologies et ont aidé le pays à accroître la part des produits de haute technologie dans ses échanges extérieurs. La prochaine section examine ces questions plus en détail.

65 Voir OCDE, “Analysing Global Interactions with Input-Output Tables”, DSTI/EAS/IND/SWP(2005)8, OCDE, Paris, 8 novembre 2005.

JETRO (2005) “Japan's Trade with China Sets Sixth Straight Year Record in 2004”, 21 février 2005 http://www.jetro.go.jp/en/news/releases/20050221305-news.

Office of Trade and Industry Information (OTII), Manufacturing and Services, International Trade Administration, ministère du Commerce des États-Unis. 


\section{Mobilité de la Chine au sein de la chaîne de valeur}

68. Au début des années 90 , le textile et les autres industries légères comptaient pour plus de $40 \%$ des exportations de la Chine. La part des secteurs plus complexes de l'électronique, de l'ameublement et des transports est passée de $17.3 \%$ des exportations totales en 1990 à $41.7 \%$ en $2004{ }^{69}$ Bien que ses exportations demeurent pour une large part composées de produits à forte intensité de main-d'œuvre, la Chine a de plus en plus diversifié ses exportations au cours des 15 dernières années au profit de biens plus complexes à forte intensité de capital et de technologie. Les données ici présentées suggèrent que la Chine poursuit une stratégie d'expansion de ses exportations sur deux fronts au lieu de se contenter d'une spécialisation conforme à l'avantage comparatif que lui confère son abondante main-d'œuvre peu qualifiée. Le premier aspect de cette stratégie consiste à tirer parti du facteur de production qui constitue son atout majeur - sa main-d'œuvre surabondante - en privilégiant les produits manufacturés à forte intensité de main-d'œuvre, qui créent des emplois. Le second consiste à promouvoir son objectif de développement économique en modernisant son économie grâce à la production et à l'exportation de biens de plus haute technologie.

69. La Chine produit et exporte des produits manufacturés à bas coût n'incorporant que peu de technologie tout en concurrençant de plus en plus les pays de l'OCDE dans le secteur des produits technologiques complexes. Une grande partie des exportations de la Chine se composent de produits manufacturés à forte intensité de main-d'œuvre peu qualifiée tels que les jouets, les textiles, les chaussures et les assemblages électroniques. Comme le montre la figure 5, les produits de basse technologie détiennent encore la plus grande part dans les exportations totales vers les pays de l'OCDE, soit 36\%. Les exportations de biens de haute technologie à destination de la zone OCDE ont cependant enregistré au cours de la dernière décennie une plus forte croissance que toutes les autres catégories de biens, puisqu'elle s'est élevée à 47\% par an de 1992 à 2003.

Figure 5. Composition des exportations de la Chine vers les pays de l'OCDE, diverses années

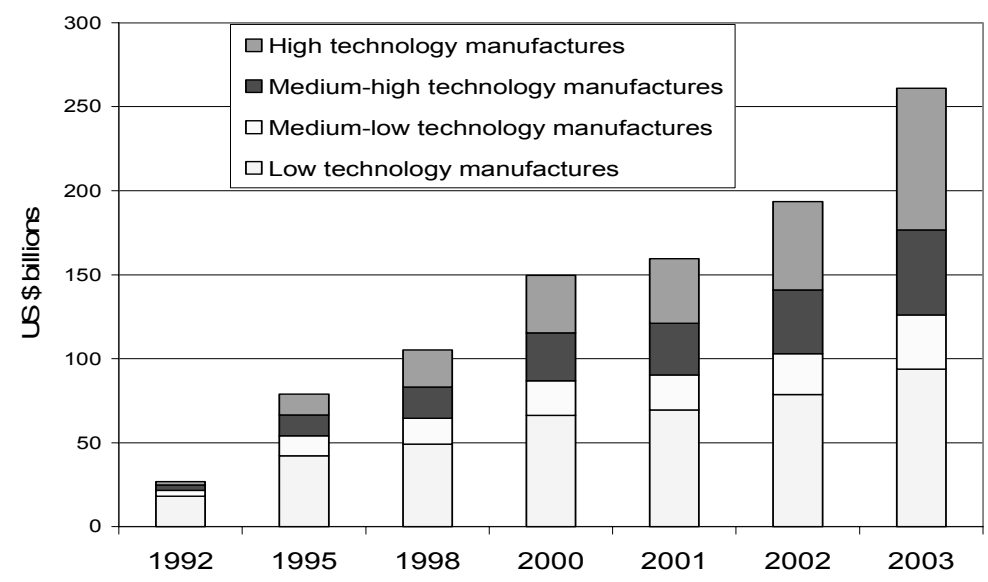

Sources: OCDE, Base de données STAN sur les échanges bilatéraux; Statistiques du commerce international par produits (ITCS), 2005. 
Figure 6. Composition des importations de la Chine en provenance des pays de l'OCDE, diverses années

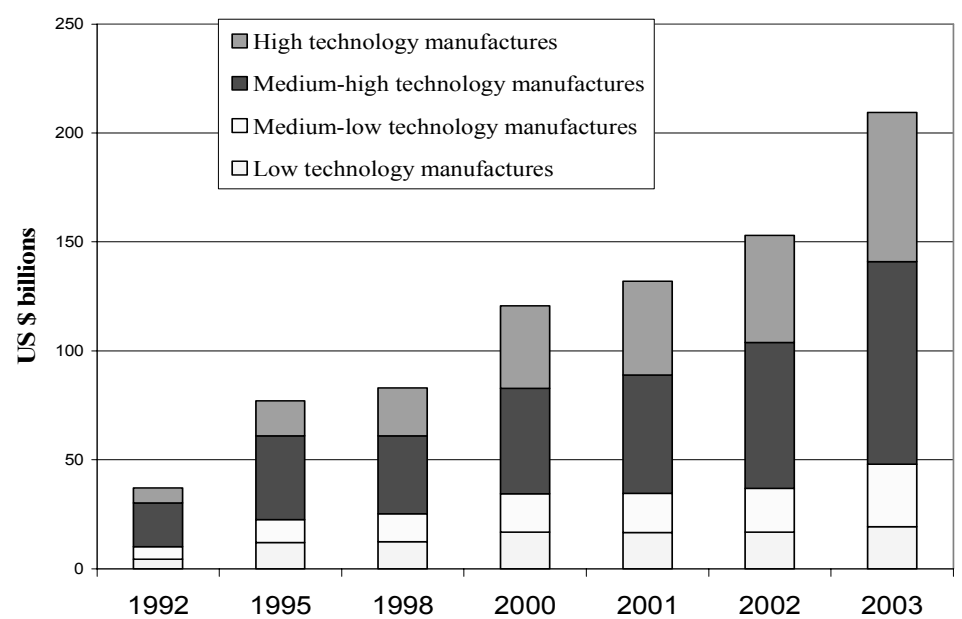

Sources: OCDE, Base de données STAN sur les échanges bilatéraux; Statistiques du commerce international par produits (ITCS), 2005.

70. Comme le montre la figure 6, les biens de haute technologie sont également la catégorie d'importations qui a enregistré la croissance la plus rapide, puisqu'elle s'est élevée à $24 \%$ par an en moyenne durant la période 1992-2003. Toutefois, quoique très dynamique, la croissance des importations de haute technologie demeure bien inférieure à celle des exportations de haute technologie, qui a atteint $47 \%$. Cela pourrait donner à penser que les exportations de biens de haute technologie comportent une part de valeur-ajoutée locale et ne se réduisent pas à la réexportation de produits n'ayant fait l'objet que de légères améliorations ou d'opérations d'assemblage.

Figure 7. Échanges de produits manufacturés entre la Chine et les pays de l'OCDE dans le secteur des TIC

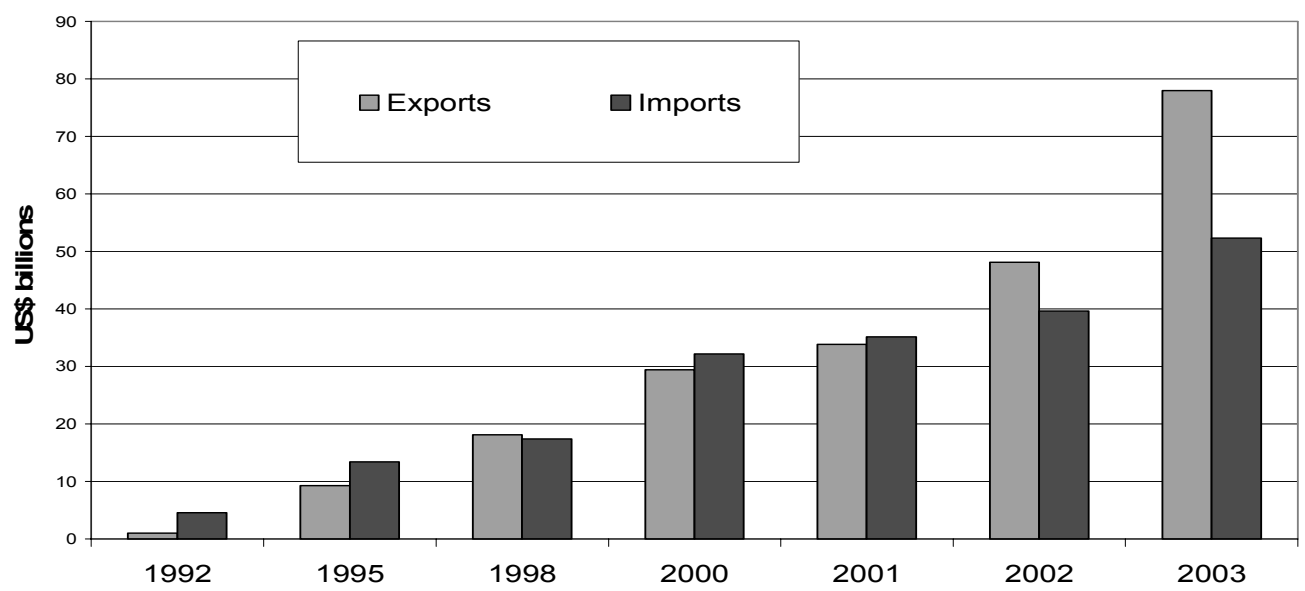

Sources : OCDE, Base de données STAN sur les échanges bilatéraux ; Statistiques du commerce international par produits (ITCS), 2005.

71. La plupart de ces exportations de haute technologie ont lieu dans les secteurs des technologies de l'information et de la communication (TIC). La Chine est devenue l'un des principaux lieux de production et d'assemblage de biens des TIC au monde. La part de la Chine dans l'ensemble des échanges mondiaux de biens des TIC ne dépassait pas 35 milliards USD en 1996. En 2004, elle a atteint près de 329 milliards USD, ce qui représente une croissance de près de 38\% par an depuis 1996. Toujours en 2004, la Chine est 
devenue le plus grand exportateur de biens des TIC, ravissant la première place aux États-Unis après avoir dépassé le Japon et 1'UE en $2003 .^{70}$ Depuis 2002, la Chine est un exportateur net de biens de haute technologie vers les pays de l'OCDE (figure 7).

72. Une importante question se pose : la Chine se contente-t-elle d'assembler les produits ou a-t-on quelque raison de croire qu'elle incorpore une plus grande valeur ajoutée dans les biens des TIC à plus forte valeur ajoutée ? Une étude de l'OCDE a constaté que les entreprises chinoises du secteur des TIC ne se contentent pas simplement d'assembler les produits et de les réexporter vers les pays de l'OCDE, mais sont en outre de plus en plus compétitives dans certaines parties du processus de production qui requièrent une main-d'œuvre qualifiée et des intrants de plus haute technologie. Les fabricants de semiconducteurs (tels que Semiconductor Manufacturing International) et les portails internet (tels que Baidu, ou le partenariat Alibaba-eBay) en sont des exemples. Enfin, les entreprises chinoises ont récemment acheté des sociétés de l'OCDE spécialisées dans les TIC ou dans les produits électroniques et les appareils électroménagers destinés au grand public (Lenovo a ainsi acquis la division ordinateur personnel d'IBM). ${ }^{71}$

73. L'analyse au niveau des produits confirme à quel point les biens de haute technologie du secteur de l'électronique occupent une place importante dans les exportations de la Chine. Huit de ses dix principaux produits d'exportation (au niveau des codes à 6 chiffres du $\mathrm{SH}$ ) relèvent du secteur de l'électronique ou de celui des communications (figure 8). Certains sont certes importés et exportés en grandes quantités, ce qui donne à penser qu'une partie des échanges pourrait être liée à des opérations d'assemblage ou de transformation à faible valeur ajoutée, mais d'autres n'en sont pas moins presque exclusivement exportés. Des exemples en sont les périphériques informatiques d'entrée et de sortie, les émetteurs de téléphonie mobile et les appareils d'enregistrement vidéophonique. Beaucoup de ces produits n'étaient pas exportés voici dix ans, ni même cinq.

Figure 8. Les 10 principaux produits d'exportation de la Chine et les intrants correspondants, en 2004

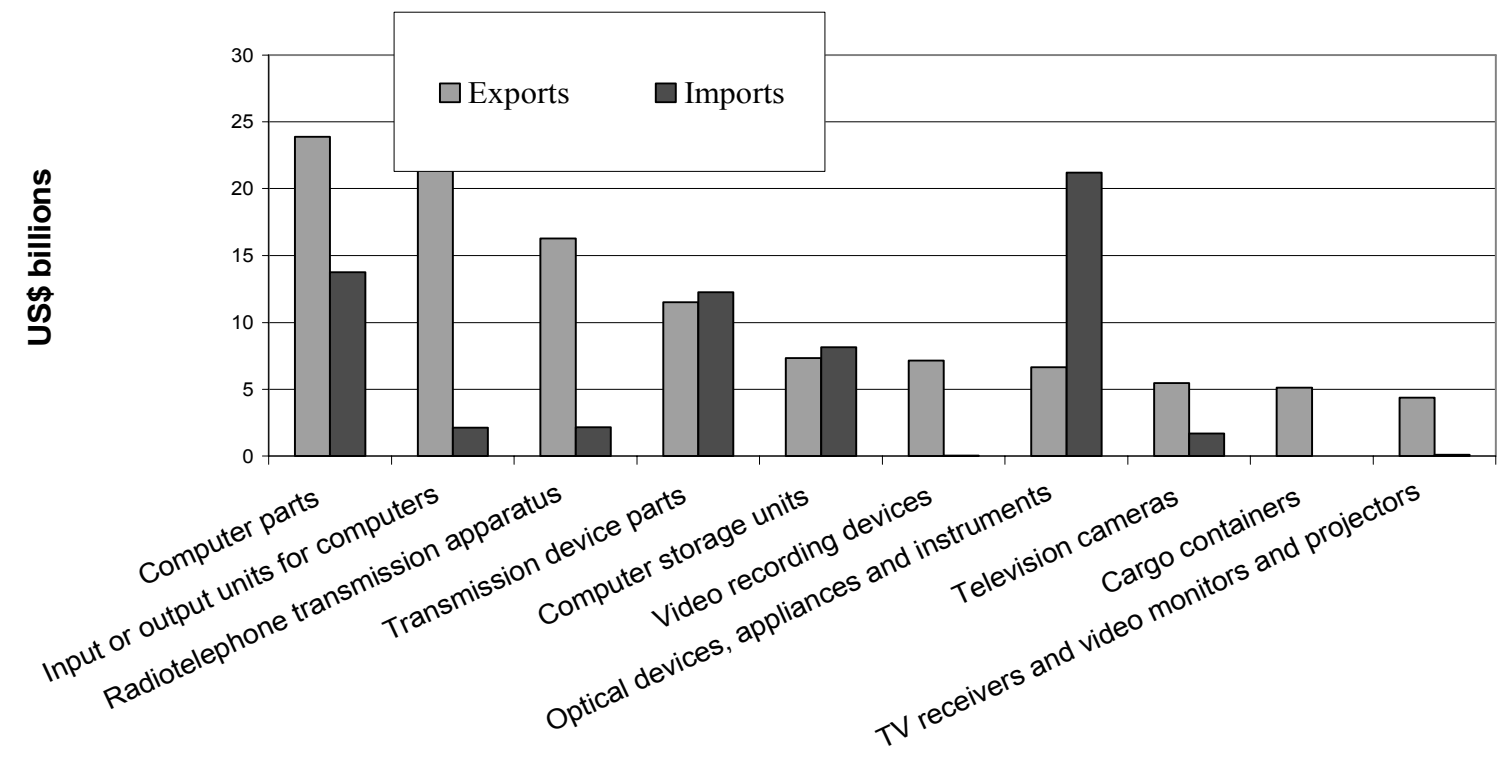

Source : OCDE, Statistiques du commerce international par produits (ITCS), 2006.

70 Voir OCDE (2005h) Information Technology Outlook 2006: China - ICT Supply, Demand and ICT Policy DSTI/ICCP/IE(2005)12/Chap4, 8-9 décembre 2005, p. 1.

$71 \quad$ Ibid., p. 2. 
Figure 9. Les 10 principaux produits chinois importés par les États-Unis

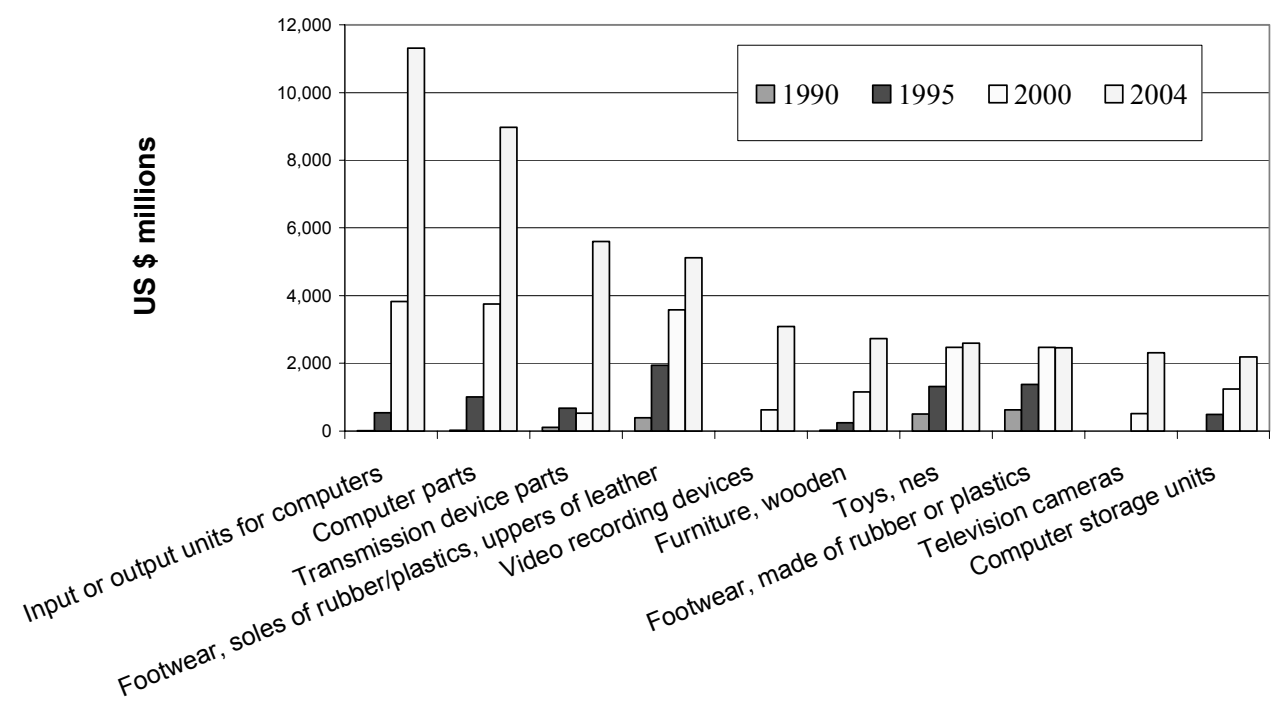

Source : OCDE, Statistiques du commerce international par produits (ITCS), 2006.

74. Aucun des 10 principaux produits chinois importés par les États-Unis en 2004 n'était importé en quantités notables en 1990 (figure 9). Parmi ceux importés en 1995, seuls certains produits de l'industrie chinoise de la chaussure continuaient de représenter une part importante des importations des États-Unis en 2004. Toutes les importations de biens des secteurs de l'électronique et des technologies de l'information ont enregistré une considérable expansion depuis 2000.

\section{La Chine est-elle en train de devenir plus compétitive?}

75. Comme l'a montré la section précédente, la Chine s'est progressivement élevée dans la chaîne de valeur ajoutée depuis le début des années 90 : après avoir d'abord mis l'accent sur les exportations de textiles, d'habillement et de jouets, elle privilégie désormais celles de matériel de transport, de machines et de produits électroniques. La Chine est-elle en train de devenir plus compétitive sur les marchés mondiaux? Molnar (2005) étudie la compétitivité de la Chine en comparant ses produits de plus haute technologie à ceux du Japon sur un marché tiers, celui des États-Unis. ${ }^{72}$ Elle constate que pour la plupart des produits les prix unitaires japonais sont au moins quatre fois supérieurs aux chinois. La part de ces produits a baissé, passant d'environ $80 \%$ en 2000 à $70 \%$ en 2004. Dans le même temps, le pourcentage des produits pour lesquels le prix unitaire chinois est proche du japonais demeure relativement réduit, bien qu'il soit en progression, puisqu'il est passé d'environ 7\% en 2000 à $10 \%$ en 2004. D'après Molnar, la diminution du pourcentage de produits caractérisés par d'importants écarts de prix unitaires et la progression constante du pourcentage de produits caractérisés par de faibles écarts de prix unitaires peuvent s'expliquer de deux façons. Les sociétés japonaises pourraient en effet être en train de baisser leurs prix pour rester compétitives. Et surtout les entreprises chinoises pourraient s'attacher de plus en plus à exporter des biens d'une qualité similaire à celle des produits japonais, s'élevant ainsi dans la chaîne de valeur et devenant par là-même plus compétitives.

76. D'aucuns soutiennent que la compétitivité de la Chine sur les marchés mondiaux est due à son taux de change favorable. La dévaluation en 1994 de la monnaie chinoise, passée de 5.8 à $8.3 \mathrm{RMB} /$ Yuan pour

72 Voir M. Molnar (2005), China Embraces Globalisation: Strengthening Integration in Trade and Investment, presented at the LACEA 2005 conference at the American University of Paris, 29 October 2005. 
un USD est souvent citée comme un facteur essentiel de l'extraordinaire essor des exportations chinoises et de leur compétitivité croissante. Arndt (2004) a constaté que le partage de la production modifie la nature des comptes de la balance commerciale et tend à réduire la sensibilité des flux commerciaux aux variations des taux de change. ${ }^{73}$ Ces considérations ne sont pas sans intérêt pour le débat sur la valeur de la monnaie chinoise et sur les effets probables d'une réévaluation du RMB/Yuan. Arndt parvient à la conclusion que la sensibilité des flux commerciaux aux variations des taux de change devrait diminuer à mesure que la part des échanges liés au partage de la production s'accroît, tant du côté des exportations que des importations.

77. Bien qu'ils constatent que la monnaie chinoise est nettement sous-évaluée, Adams et al. (2006) font valoir que d'autres facteurs contribuent également à la compétitivité accrue de la Chine. ${ }^{74}$ Il s'agit en premier lieu de l'important afflux d'IDE et de cadres étrangers. L'IDE pourrait avoir apporté la plus grande contribution à la compétitivité au travers de l'introduction de nouvelles méthodes de production, des spécifications de produits en vigueur sur les marchés mondiaux et des procédures de gestion les plus modernes. Entrent également en ligne de compte les bas salaires chinois et l'offre abondante de maind'œuvre non qualifiée, ainsi que les coûts réduits de communication et de transport. ${ }^{75}$

\section{Les exportations de la Chine sont plus proches de celles de la Corée que de celles de l'Inde}

78. Dans une étude récente, Rodrik (2006) compare la composition des exportations de différents pays en fonction du niveau de leur revenu par habitant et constate que les exportations de la Chine sont comparables à celles de pays disposant d'un revenu par habitant trois fois supérieur au sien. Les exportations de la Chine ont évolué depuis le début des années 90 : d'abord plutôt proches de celles de l'Inde, elles le sont désormais davantage de celles de la Corée ou de l'économie de Hong Kong, Chine. ${ }^{76}$ On ne s'attendrait pas en règle générale à ce qu'un grand pays pauvre doté d'une main-d'œuvre abondante comme peut l'être la Chine produise, et encore moins exporte, un large éventail de produits de haute technologie. Rodrik souligne que ce qui est si frappant dans le cas de la Chine, ce ne sont pas tant le volume de ses exportations ni son vaste réservoir de main-d'œuvre, qui lui permet de bénéficier d'un énorme avantage en termes de coûts du facteur travail, mais bien le fait qu'elle produise et vende des produits associés à un degré de productivité nettement supérieur à celui des pays disposant d'un niveau de revenu comparable au sien. Il en attribue le mérite aux politiques gouvernementales qui ont contribué à développer les capacités de production chinoises dans l'électronique grand public comme dans le domaine des autres produits de pointe.

79. Rodrik soutient qu'il n'est nullement assuré que le modèle de croissance axé sur les exportations mis en œuvre par la Chine puisse s'inscrire dans la durée. Les produits de haute technologie ont été les secteurs d'exportation les plus dynamiques en Chine et ont favorisé la forte croissance des exportations du pays. Compte tenu de la part si élevée et en constante augmentation que représentent les exportations dans le PIB, Rodrik examine dans quelle mesure le modèle de croissance chinois paraît être en train de s'essouffler. Il part du postulat que l'essentiel, pour juger de la durabilité de sa stratégie de croissance axée sur les exportations, c'est de savoir si la Chine sera à même de s'élever dans la chaîne de valeur pour produire des biens plus rémunérateurs afin d'alimenter et de maintenir des taux de croissance élevés. ${ }^{77} \mathrm{Il}$ apparaît que ce qui importe pour la croissance future de la Chine, ce n'est pas tant le volume des

73 Arndt (2004), op. cit.; voir également l'analyse de D. Rodrik, (2006), "What's so special about China's exports?" document de travail du NBER n 11947, Cambridge, MA, janvier 2006.

F. G. Adams, B. Ganges et Y. Shachmurove, "Why is China so Competitive? Measuring and Explaining China's Competitiveness", World Economy, vol. 29, n 2, février 2006.

75 Ibid., p. 120.

76 Rodrik (2006), op. cit., p. 11.

$77 \quad$ Ibid., p. 24. 
exportations ou leur part dans le PIB que leur "qualité". On soutiendra ici que la Chine en a parfaitement conscience et poursuit par conséquent une stratégie d'expansion de ses exportations sur deux fronts en privilégiant les produits à forte intensité de main-d'œuvre afin de tirer parti de sa main-d'œuvre abondante tout en mettant en œuvre des politiques gouvernementales favorisant l'essor des biens de haute technologie en vue de soutenir sa stratégie de développement économique.

80. Dans le $11^{\text {ème }}$ Plan quinquennal, la Chine vise à protéger les DPI en vue de stimuler l'innovation en tant qu'elle constitue la condition essentielle d'une amélioration de la compétitivité. ${ }^{78}$ La prochaine section explique pourquoi la protection des DPI est importante pour le développement économique de la Chine.

\section{DPI et transfert de technologies}

81. Le bien-être économique d'un pays est étroitement lié aux ressources dont il dispose et au degré de productivité de l'utilisation qui en est faite. Le progrès technologique joue un rôle crucial dans l'augmentation du volume de production par salarié, et il constitue un important déterminant des niveaux de revenu. ${ }^{79} \mathrm{Hu}$ et Khan (1997) ont examiné le rapide développement économique de la Chine et ils ont constaté que les gains de productivité ont joué un rôle important dans la croissance, puisqu'ils ont contribué pour plus de $50 \%$ à l'augmentation de la production au début des années 90 . D'après leurs observations, outre l'innovation intérieure, un facteur a contribué à accroître la productivité, à savoir en l'occurrence la politique de porte ouverte qui a permis d'avoir accès aux marchés comme aux investissements internationaux, d'où d' "importants transferts de technologies". Les améliorations apportées à la protection de la propriété intellectuelle en Chine accroissent les incitations économiques à rendre accessibles de nouvelles technologies d'origine nationale ou internationale.

82. Les mesures prises pour assurer une protection satisfaisante de la propriété intellectuelle peuvent avoir de considérables effets sur une économie en raison pour partie des caractéristiques particulières de ces actifs. Contrairement aux ressources matérielles, un même élément de propriété intellectuelle peut être mis de manière simultanée et répétée à la disposition non exclusive de multiples utilisateurs, généralement à un faible coût marginal. Du fait de leur "non-rivalité", les idées nouvelles couvertes par la propriété intellectuelle peuvent non seulement contribuer au progrès technologique mais aussi avoir des effets "disproportionnés" sur la croissance économique vu leurs rendements d'échelle élevés lorsqu'elles sont mises en application bon nombre de fois [Jones (2004)]. Compte tenu de ce potentiel économique, les décideurs chinois ont à juste titre cherché à améliorer le régime appliqué aux DPI et encouragent par làmême l'accès à un plus large stock de propriété intellectuelle ainsi qu'aux technologies sous-jacentes.

83. Les avantages pour la Chine d'une politique encourageant efficacement le transfert de technologies, grâce notamment au respect des obligations contractées en vertu de l'Accord sur les ADPIC de l'OMC, peuvent être tout à fait considérables compte tenu du fossé qui existe entre les technologies en usage dans certains secteurs au sein même du pays et celles à la pointe du progrès qui sont disponibles sur les marchés mondiaux. Aussi, la Chine se trouve-t-elle, comme l'ont souligné Mu et Lee (2005) et quelques autres, exceptionnellement bien placée pour bénéficier d'un rattrapage technologique. L'immensité de son marché intérieur lui confère en effet une position extrêmement favorable dans la négociation de contrats internationaux de transfert de technologies (grâce par exemple à l'échange de parts de marché contre des technologies dans le cadre des coentreprises). Il reste dans le même temps un considérable écart à combler sur le plan technologique (d'où des gains économiques potentiels d'autant plus importants), puisque la Chine est à certains égards en retard de ce point de vue. Elle est dans certains cas en situation de sauter des

\footnotetext{
78 Voir "IPR Protection to Encourage Innovation", China Daily, 16 février 2006, http://www.china.org.cn/english/China/158156.htm.

79 Pour un examen de cette question et davantage de références bibliographiques, voir : OMC (2002), Commerce et transfert de technologie, WT/WGTTT/W/1, Organisation mondiale du commerce, Genève, 2 avril 2002.
} 
générations entières de technologies plus anciennes. Par exemple, dans les télécommunications, la Chine a pour une large part pu éviter de mettre en place des réseaux téléphoniques basés sur l'usage de commutateurs analogiques pour passer directement à l'utilisation de commutateurs numériques.

84. Les recherches axées sur les pays en développement confirment qu'une bonne politique en matière de DPI peut élargir l'accès aux technologies. Lorsqu'un pays en développement est à la traîne pour ce qui est de l'application de certaines technologies du fait qu'il n'est guère possible de se les procurer auprès de fournisseurs intérieurs, les détenteurs étrangers de propriété intellectuelle peuvent considérablement l'aider à réduire son retard [Park et Lippoldt (2003)]. Les entrées de biens, d'investissements directs et de licences incorporent divers types de propriété intellectuelle et constituent des vecteurs potentiels de transfert de technologie. Ces entrées peuvent en outre être influencées par l'environnement en place dans une économie concernant les DPI. Park et Lippoldt (2005) ont souligné l'importance des licences internationales en tant que vecteur de transfert de technologies. Ils ont en effet constaté que d'importants flux de technologie entrent déjà en Chine sous licence (en particulier en provenance des États-Unis et du Canada). Une nouvelle amélioration du système de DPI en vigueur en Chine pourrait permettre d'en accroître le volume. Pour ce qui est des pays en développement, Park et Lippoldt ont observé que la réforme des DPI paraît exercer une influence sur ces transferts de technologie. Les droits de brevets et leur protection efficace peuvent notamment être essentiels pour permettre aux entreprises des pays en développement d'avoir accès aux technologies et aux savoir-faire en vue de les exploiter au travers d'accords de licence avec des partenaires établis dans les pays développés. L'analyse tend à montrer que les pays en développement qui ont pris des mesures ces dernières années pour remédier aux déficiences dans ces domaines ont en règle générale bénéficié d'un meilleur accès aux technologies grâce au système de licences.

\section{Effets de la Chine sur les prix mondiaux}

85. La participation croissante de la Chine aux échanges internationaux a eu d'importantes répercussions sur les prix relatifs. ${ }^{80}$ Lorsqu'un grand pays tel que la Chine fournit des quantités additionnelles d'un certain produit sur les marchés mondiaux, il en résulte une baisse du prix mondial de celui-ci. De la même façon, lorsque ledit grand pays accroît ses importations, il s'ensuit une hausse des prix mondiaux. C'est précisément ce qui s'est produit au cours des toutes dernières décennies. La Chine est importateur net de matières premières et exportateur net de biens manufacturés, et elle est suffisamment vaste pour exercer une pression sur les prix des unes comme des autres. D'une part, les importations en forte augmentation de la Chine sont pour une large part constituées de produits de base essentiels et ont contribué à la vigueur des cours mondiaux de ces derniers. D'autre part, le rapide essor des exportations chinoises d'un vaste et complexe éventail de produits finis exercent une pression à la baisse des prix mondiaux de ces produits, tels que les textiles et l'habillement, l'acier, les appareils électroménagers grand public, et les pièces automobiles. Certains se sont inquiétés de ce que la Chine puisse être en train d'exporter la déflation vers d'autres pays en exerçant une pression à la baisse des prix du marché. ${ }^{81}$

\footnotetext{
$80 \quad$ Voir également l'encadré I.5 sur la mondialisation et l'inflation in Perspectives économiques de l'OCDE $n^{\circ} 79$, 2006.

81 Feyzioglu et Willard évaluent dans quelle mesure il existe une relation entre les taux d'inflation de la Chine, des États-Unis et du Japon. Ils ne trouvent que peu de données empiriques au niveau agrégé tendant à montrer que l'inflation des prix à la consommation en Chine entraîne des variations de prix aux États-Unis et au Japon. Ils constatent toutefois que certaines données paraissent indiquer que l'inflation enregistrée aux États-Unis a une incidence sur celle observée en Chine. Ils affirment que cette incidence est de courte durée. Voir T. Feyzioglu et L. Willard (2006), "Does Inflation in China Affect the US and Japan", Document de travail du FMI, WP/06/36, FMI, Washington, DC, février 2006.
} 
Figure 10. Prix mondiaux des produits primaires

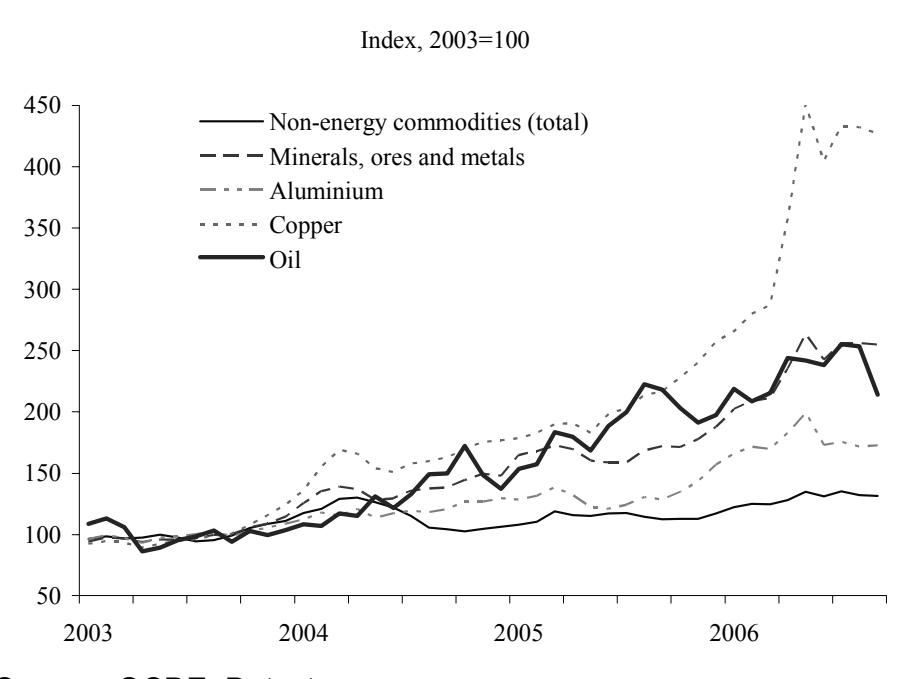

Source : OCDE, Datastream.

86. Du fait de son rapide processus d'urbanisation, d'industrialisation et de construction d'infrastructures, la Chine importe des quantités toujours croissantes de matières premières et de produits primaires, d'où des pressions à la hausse sur les prix mondiaux des principaux produits de base (figure 10). La récente tendance à la hausse des prix des produits de base est imputable à la très forte demande en Chine mais aussi en Inde, ainsi qu'aux problèmes qui commencent à se faire jour du côté de l'offre (CNUCED, 2005b). Le gouvernement chinois a déployé des efforts concertés pour s'assurer des sources futures d'approvisionnement en pétrole, gaz naturel, minerais de fer, aluminium, bois d'œuvre et autres produits de base en Australie, en Russie et dans les pays en développement d'Afrique et d'Amérique latine. $^{82}$

87. La demande d'acier de la Chine mérite de faire l'objet d'un examen plus approfondi et offre un bonne illustration de l'augmentation de la demande et de ses effets sur les prix mondiaux. La Chine est devenue le premier consommateur et le premier producteur d'acier en 1996. La demande d'acier chinoise a progressé très régulièrement entre 1970 et 1992, enregistrant une augmentation annuelle moyenne de $7.7 \%$. La demande intérieure d'acier ne s'est en moyenne accrue que de $1,6 \%$ par an jusqu'en 2000, date à laquelle elle a totalisé 121.2 millions de tonnes. ${ }^{83}$ L'OCDE (2005i) a constaté qu'à partir de 2001 "la demande d'acier s'est emballée et elle n'a cessé de croître à un rythme très élevé, $+26.2 \%$ en 2001 , $+21.4 \%$ en $2002,+25.6 \%$ en 2003 et $+10 \%$ en 2004 , année où la consommation d'acier s'est établie à 256.6 millions de tonnes." Il était à l'en croire vraisemblable que la consommation d'acier chinoise soit en 2005 proche de 300 millions de tonnes. Dans le même temps la production d'acier brut en Chine est passée de 46.8 millions de tonnes en 1985 à 272.5 millions de tonnes en 2004. En 2005, la production d'acier pourrait atteindre 340 millions de tonnes, et la Chine devrait devenir pour la première fois en 2005 exportateur net d'acier. ${ }^{84}$

88. La Chine craint la "surchauffe" et a lancé un vaste programme de rationalisation de sa sidérurgie. ${ }^{85}$

82 La Chine est désormais le troisième importateur, derrière les États-Unis et l'UE, de produits exportés par les pays en développement. En 2003, les importations chinoises en provenance d'Amérique latine ont augmenté de $81 \%$ alors que celles originaires d'Afrique ont progressé de $51 \%$.

83 Voir OCDE (2005i), "Évolution des capacités mondiales d'acier", [DSTI/SU/SC(2005)15], OCDE, Paris, 21 octobre 2005 .

Ibid., p. 15.

85 EIU (2006) China Country Profile 2006, p. 36. 
En vertu du plan élaboré par le gouvernement, les plus petites usines sidérurgiques doivent fusionner en vue d'accroître leur efficience et donc leur rentabilité. Le gouvernement chinois semble souhaiter limiter le développement de nouvelles capacités additionnelles, ayant un temps misé sur l'amélioration de la qualité et la diversification des produits, et il est en train de fermer de nombreuses installations obsolètes. Le gouvernement chinois concentre ses efforts sur le développement de nouvelles installations de transformation pour augmenter sa production de produits sidérurgiques à forte valeur ajoutée. L'EIU note que les responsables gouvernementaux ont été contraints de prendre des mesures à plus court terme à la suite d'une spectaculaire baisse des prix de l'acier chinois en septembre 2005, date à laquelle les prix de l'acier laminé à chaud ont chuté de $50 \%$ par rapport à leur plus haut niveau de cette même année. Lors d'une réunion en octobre 2005 de l'association chinoise des industries sidérurgiques (China Iron and Steel Industry Association), 45 des principaux producteurs d'acier ont préconisé une concentration plus rapide du secteur et la formation d'un groupe industriel en vue d'assurer une meilleure adéquation entre l'offre et la demande. ${ }^{86}$

89. La CNUCED (2005b) note cependant que, malgré la montée en flèche de la demande chinoise, il demeure improbable qu'il en résulte un retournement définitif de la tendance à la baisse des prix réels des produits de base. Ceux-ci demeurent en effet inférieurs de plus de un tiers à leur niveau moyen sur la période 1960-1985. ${ }^{87}$ Néanmoins, cet accroissement de la demande chinoise de matières premières et de produits primaires a à la fois des effets positifs et négatifs sur l'économie mondiale. La demande accrue est bénéfique pour les producteurs de ces biens du fait de l'augmentation des volumes d'exportation et de la hausse des prix mondiaux. Cette hausse des prix a toutefois des conséquences négatives pour les importateurs de matières premières et de produits primaires.

90. Pour ce qui est des exportations, la poursuite de la réduction des obstacles au commerce et la rapide augmentation de la productivité dans le secteur manufacturier et celui de l'électronique en Chine ont abouti à une soudaine explosion des exportations de divers types de produits, d'où une baisse de leur prix sur les marchés internationaux. Les données montrent que les prix à l'exportation des textiles et de l'habillement en provenance des pays en développement ont diminué de plus de $7 \%$ depuis le milieu des années $90{ }^{88} \mathrm{La}$ baisse des prix a été encore plus forte depuis le début des années 90 pour ce qui est des produits électroniques, y compris les équipements informatiques et de télécommunications. Cette baisse est en grande partie due à l'abondance de main-d'œuvre peu qualifiée dont disposent les pays exportateurs et plus particulièrement la Chine.

91. Kaplinski (2005) a suivi l'ampleur de la baisse des prix des produits importés par l'UE, au niveau des codes à 8 chiffres du SH, au cours de la période 1988-2000 (l'UE est suffisamment vaste pour donner une idée approximative du comportement des prix mondiaux des produits). ${ }^{89}$ La figure 11 présente les constations de Kaplinski : dans presque un tiers des secteurs, les prix des produits d'origine chinoise ont diminué. Kaplinski en conclut qu'il est d'autant plus probable que les prix baissent à mesure que la participation de la Chine aux marchés mondiaux de produits s'accroît.

\footnotetext{
$86 \quad$ Ibid.

87 CNUCED (2005b), op. cit., p. iii.

88 Ibid., p. 88.

89 R. Kaplinski (2005), Globalisation, Poverty and Inequality: Between a Rock and a Hard Place, Cambridge: Polity Press, 2005.
} 
Figure 11. Pourcentage des secteurs confrontés à une tendance à la baisse des prix au sein de l'UE, par catégories de pays

$(1988-2000)$

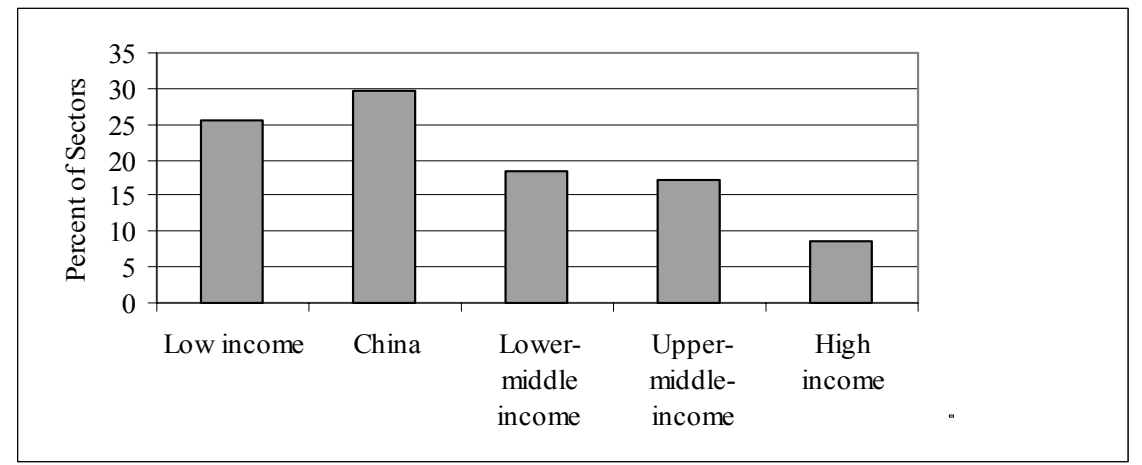

Source : Kaplinski (2005).

Figure 12. Secteurs d'exportation confrontés à une baisse des prix aux États-Unis

(Pourcentage)

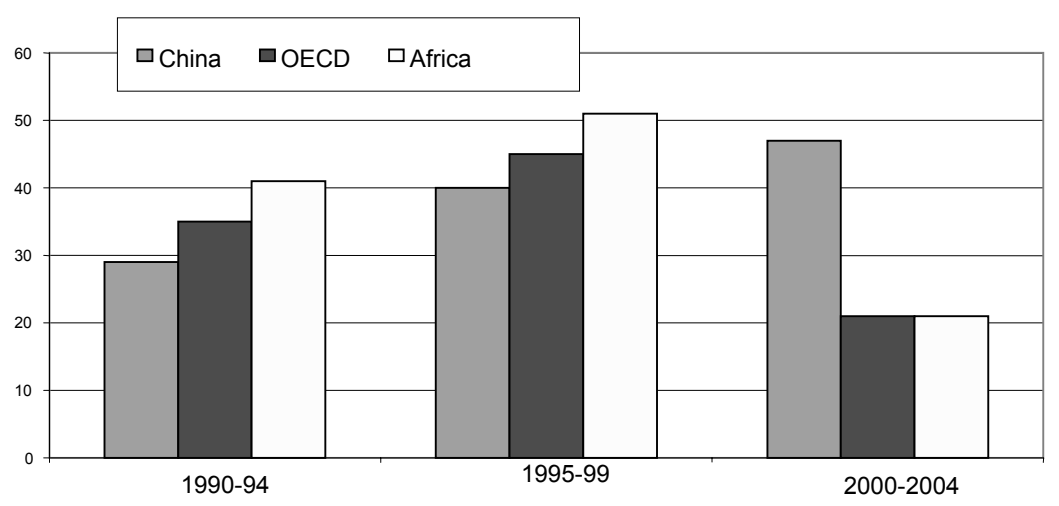

Source : Statistiques du commerce extérieur de l'OCDE.

92. Afin de mieux mettre en évidence les effets de la baisse des prix des exportations chinoises, la figure 12 compare les exportations à destination des États-Unis en provenance de la Chine, des pays de l'OCDE et des pays d'Afrique au cours des 15 dernières années au niveau des codes à 6 chiffres du SH. Entre 1990 et 1994, 29\% des exportations chinoises vers les États-Unis ont enregistré une baisse des prix, contre $35 \%$ pour celles des pays de l'OCDE et $41 \%$ pour celles des pays d'Afrique. De 1995 à 1999, les pourcentages sont d'environ $10 \%$ plus élevés mais la structure d'ensemble demeure la même. On observe par contre une nette différence pour ce qui est de la période allant de 2000 à 2004. Chose intéressante, le pourcentage des exportations des pays de l'OCDE et des pays d'Afrique qui enregistrent une baisse des prix tombe à $21 \%$ alors que celles de la Chine continuent à augmenter, jusqu'à atteindre $47 \%$.

93. La Chine a subi une baisse des prix des produits qu'elle exporte et une hausse des prix de ceux qu'elle importe. L'impact de la dégradation des termes de l'échange s'accroît à mesure que la part relative du commerce extérieur dans le PIB augmente, comme tel est le cas en Chine. Le principal défi consistera pour la Chine à savoir faire face à la dégradation de ses termes de l'échange à mesure que se poursuivent l'expansion de son commerce extérieur et sa croissance économique, d'où un impact d'autant plus fort sur les prix internationaux de ses importations et de ses exportations. 


\section{Les termes de l'échange de la Chine et leur effet sur l'économie mondiale}

94. Les considérations ci-dessus ont permis de dégager deux grandes tendances - une hausse des prix des produits importés par la Chine et une baisse des prix de ses exportations - qui se prolongeront probablement à mesure que se poursuivra son processus de "rattrapage" de développement. La conjonction de ces deux tendances entraîne une dégradation des termes de l'échange de la Chine (voir le tableau 13). Cependant, pour un pays comme la Chine, cette détérioration des termes de l'échange peut ne pas comporter les mêmes risques que pour les pays en développement de plus petite taille ou pour ceux dont les échanges sont pour une plus grande part constitués de produits primaires. Certains affirment que la dégradation des termes de l'échange de la Chine peut être compensée par la structure de ses exportations et par leur volume croissant ainsi que par la compétitivité internationale de ses producteurs. Les termes de l'échange-revenu ou le pouvoir d'achat des exportations (défini comme la valeur de l'indice des exportations divisé par la valeur unitaire des importations) pourraient dès lors mieux expliquer la capacité de la Chine à importer des biens essentiels pour son développement. La Chine pourrait ainsi compenser la baisse de ses termes de l'échange par une augmentation du volume de ses exportations. La demande internationale croissante et une augmentation de la part du marché mondial détenue par la Chine ont pour effet d'accroître le volume de ses exportations. La Chine pourrait donc bénéficier d'une hausse du pouvoir d'achat de ses exportations, ce qui indique qu'elle pourrait être capable d'accroître ses importations réelles sans conséquences négatives pour sa balance commerciale. ${ }^{90}$

Tableau 13. Évolution des termes de l'échange de la Chine

$(2000=100)$

\begin{tabular}{lcccc}
\hline & $\mathbf{1 9 8 4}$ & $\mathbf{1 9 9 4}$ & $\mathbf{2 0 0 3}$ & $\mathbf{2 0 0 4}$ \\
Export price index & 50 & 103 & 96 & 102 \\
Import price index & 74 & 96 & 102 & 112 \\
Terms of trade & 68 & 107 & 95 & 91 \\
\hline Source : Banque mondiale, Indicateurs du développement dans le monde
\end{tabular}

Source : Banque mondiale, Indicateurs du développement dans le monde

95. Lors de l'étude de l'impact de la Chine sur les prix mondiaux, il convient également de bien faire la distinction entre la situation de l'offre et celle de la demande au sein du pays. Bien que les analystes ne s'accordent pas sur le point de savoir dans quelle mesure la Chine sera capable de soutenir des taux de croissance aussi remarquables à l'avenir, l'idée qu'elle continuera d'enregistrer une croissance rapide, à un rythme plus élevé que l'économie mondiale dans son ensemble, suscite quant à elle un large accord. Or, vu que la Chine compte pour près de un cinquième de la population mondiale, cela donne à penser que la demande mondiale demeurera probablement robuste à court comme à moyen terme.

96. En ce qui concerne l'offre, il s'agit de savoir si la Chine commencera à se heurter à des contraintes de capacité qui réduiront ses possibilités d'exercer une pression à la baisse des prix mondiaux des produits manufacturés. La réponse à cette question sera dans une large mesure fonction de la conviction, ou non, que la Chine finira par connaître des pénuries de main-d'œuvre qui exerceront une pression à la hausse des salaires et qui pourraient donc ralentir la croissance de ses exportations manufacturières. Les études sur la question indiquent "que les salaires ne devraient pas augmenter en Chine à moyen terme, du moins dans les industries manufacturières à vocation exportatrice, qui ont la possibilité de transférer leurs activités vers l'intérieur du pays et de recruter ses salariés en puisant dans la masse de chômeurs et de personnes sousemployées que recèlent les zones rurales" (Kaplinski 2005). Il s'agit dès lors de savoir s'il en résultera des pressions déflationnistes sur l'économie mondiale.

97. La croissance économique et la politique commerciale de la Chine ont une incidence directe sur ses termes de l'échange mais aussi sur celui des autres pays. Depuis 2002, les économies dont une bonne partie

$90 \quad$ CNUCED (2005b), op. cit., p. 90. 
des importations sont constituées de pétrole, de minéraux et de produits miniers ont tiré les plus grands gains des récentes évolutions des marchés internationaux de produits du fait de la hausse des prix sur ces derniers. Dans le même temps, les pays en développement importateurs de combustibles ont subi une dégradation des termes de l'échange.

\section{Section III. Impact de l'intégration de la Chine sur certaines économies de l'OCDE, une évaluation quantitative}

98. Cette section vise à quantifier les répercussions sur le bien-être de l'intégration de la Chine dans les échanges mondiaux de biens et services. Etant donné que la croissance récente de la Chine a été dominée par les investissements, elle met plus particulièrement l'accent sur les politiques adoptées par la Chine en matière d'investissement direct étranger ainsi que sur les entrées d'IDE dont bénéficie le pays. Elle analyse en détail l'impact des stratégies de libéralisation de la Chine sur les termes de l'échange, les flux commerciaux et le bien-être des pays pris en considération. Les effets sur le bien-être et la décomposition des facteurs qui y contribuent, tels que les effets sur l'efficience allocative, sur les termes de l'échange, sur la dotation nette en capital, sur la diversité des produits et sur les revenus nets tirés de l'IDE, sont examinés aussi bien au niveau sectoriel qu'à celui de l'économie dans son ensemble.

99. L'annexe 2 offre un tour d'horizon des travaux consacrés à l'étude de l'impact que l'intégration de la Chine dans l'économie mondiale exerce sur cette dernière, afin de fournir une vue d'ensemble des estimations disponibles et des modèles utilisés pour les obtenir. L'examen montre que l'accession de la Chine à l'OMC ou la libéralisation unilatérale à laquelle elle a procédé génèrent des gains de bien-être globaux quelles que soient les hypothèses de modélisation retenues. Les résultats sont difficiles à comparer eu égard aux différences tenant à la structure des modèles, aux chocs liés aux politiques mises en œuvre et aux données relatives au degré de protection utilisées dans ces simulations. Certaines conclusions n'en peuvent pas moins être tirées.

100. Une importante conclusion s'en dégage, à savoir que la Chine est le principal gagnant quelles que soient les hypothèses de modélisation retenues - elle enregistre en effet d'importants gains de bien-être allant de 0.4 à $22.5 \%$ de son PIB. Les études dynamiques qui prennent en considération les effets additionnels liés à l'investissement font apparaître des gains plus importants, tout comme celles qui tiennent compte des améliorations de la productivité associées à la libéralisation de la Chine. Par contre, l'impact sur le reste du monde en général et sur les pays de l'OCDE en particulier demeure limité dans toutes les études analysées (il est presque toujours inférieur à 1\% de variation du PIB).

\section{Modélisation de l'impact de l'intégration de la Chine sur les économies de l'OCDE}

101. Il importe de noter d'emblée que, bien que de nombreuses études d'équilibre général se soient attachées à quantifier les conséquences économiques des politiques qui influent sur les échanges de marchandises en Chine, relativement peu de travaux ont été réalisés en vue d'évaluer les gains potentiels qui pourraient résulter d'autres scénarios de libéralisation dans le secteur des services. ${ }^{91}$ Les difficultés

91 Un certain nombre d'études qui évaluent l'impact de la libéralisation des services incluent la Chine dans l'échantillon des pays examinés. Dee et Hanslow (2000) analysent ainsi divers scénarios de libéralisation multilatérale des services à l'aide du modèle FTAP. Ils constatent des gains de bien-être d'environ 130 milliards USD (soit $0.46 \%$ du revenu réel mondial) imputables à la libéralisation des échanges de services, dont plus de 90 milliards pour la Chine (ce qui représente environ 14.6\% de son PIB réel). À partir du même type de modèle et des mêmes estimations des obstacles aux échanges de services que Dee et Hanslow, Verikios et Zhang (2000) élargissent l'analyse en fournissant des données plus détaillées au niveau sectoriel. Cette étude procède séparément à une simulation des effets d'une totale libéralisation multilatérale des échanges de services de communication et de services financiers dans le sillage du Cycle d'Uruguay et constate qu'il en résulte dans l'un et l'autre cas des effets positifs sur le bien-être pour le monde dans son ensemble. D'après les projections, une libéralisation des échanges de services de communication permettrait au 
rencontrées tiennent au peu d'informations dont on dispose au sujet des transactions internationales dans le domaine des services et des obstacles auxquels se heurtent actuellement les échanges de services, ainsi qu'à la nécessité d'élaborer une structure de modélisation différente de celle utilisée pour le commerce de marchandises, afin de prendre en considération les divers modes de fourniture des services (c'est-à-dire pour tenir compte des mouvements des facteurs de production).

102. Étant donné que la croissance récente de la Chine a été dominée par les investissements, le modèle employé dans le présent document met particulièrement l'accent sur les politiques de la Chine en matière d'IDE ainsi que sur l'examen des autres conséquences de la libéralisation par la Chine de ses échanges de services et de la levée des obstacles à l'investissement étranger au sein du pays. Le modèle tente d'analyser en détail les effets spécifiquement liés aux services et à l'investissement, ainsi que leurs interactions avec les incidences de la libéralisation des échanges de marchandises.

103. On trouvera ci-dessous une brève description du modèle FTAP utilisé dans l'étude. Elle indique quelles sont les données sous-jacentes et expose le cadre d'analyse appliqué aux scénarios de libéralisation. Les résultats de l'analyse sont ensuite examinés. Les annexes 3 et 4 présentent plus en détail la structure des échanges de la Chine avec les pays de l'OCDE et la structure théorique du modèle FTAP.

104. Afin de prendre en considération dans l'analyse les aspects relatifs aux services et à l'investissement et d'accroître la fiabilité des résultats concernant l'impact que l'intégration de la Chine dans l'économie mondiale exerce sur cette dernière, le présent document fournit de nouvelles estimations établies à l'aide d'un modèle d'équilibre général calculable de l'économie mondiale qui tient compte d'une multiplicité de pays et de secteurs, partant de l'hypothèse de rendements d'échelle croissants et d'une situation de concurrence monopolistique entre de grands groupes dans tous les secteurs, l'investissement direct étranger étant traité sur une base bilatérale (à savoir en l'occurrence à l'aide du modèle FTAP d'analyse de l'investissement direct étranger et des échanges). Vu l'importance du rôle de l'investissement direct étranger au sein du pays, il est essentiel de prendre en considération l'IDE dans un modèle EGC si l'on veut comprendre l'économie chinoise et l'impact de sa libéralisation sur l'économie mondiale. Le modèle FTAP utilisé pour les besoins de cette étude a été élaboré par étapes successives à partir du modèle GTAP, en lui adjoignant les éléments nécessaires à l'analyse de la libéralisation des services et en particulier celle de la suppression des obstacles à 1 'IDE dans le secteur tertiaire. ${ }^{92}$ Le cadre de modélisation employé dans ce document est présenté plus en détail à l'annexe 4.

\section{Données}

105. Le modèle FTAP a été adapté sous plusieurs aspects pour les besoins de la présente étude. Premièrement, cette étude s'appuie sur une base de données sectorielle plus détaillée que celles précédemment fondées sur le modèle FTAP, ce qui permet une analyse plus précise de la libéralisation des échanges de marchandises comme du commerce de services. La version provisoire de la base de données GTAP 6.1 et une nouvelle base de données sur les stocks bilatéraux de capitaux constituée dans le cadre de ce projet ont été utilisées pour mener à bien cette analyse.

monde dans son ensemble d'enregistrer une progression du revenu réel d'environ 13 milliards USD, soit une augmentation de $0.05 \%$, dont 4.4 milliards au profit de la Chine, alors que dans le cas d'une libéralisation des échanges dans le domaine de la finance, de l'assurance et des services aux entreprises, les gains escomptés s'élèveraient à environ 3.5 milliards USD, soit une augmentation de $0.01 \%$ du revenu réel, dont plus de 2 milliards reviendraient à la Chine.

92 Pour plus d'informations, voir K. Hanslow, T. Phamduc, et G. Verikios (1999) The Structure of the FTAP Model, Research Memorandum MC-58, Productivity Commission, Canberra, 1999. Le modèle FTAP et sa documentation sont disponibles sur le site web de la Commission de la productivité d'Australie (Australian Productivity Commission), dont l'adresse est la suivante : http://www.pc.gov.au. 
106. La base de données GTAP 6.1 couvre 57 grands secteurs économiques et 92 pays et tient pleinement compte des informations sur les droits bilatéraux ad valorem (NPF et préférentiels), les équivalents ad valorem des droits spécifiques (NPF et préférentiels) et les contingents tarifaires tirées de la base de données MacMaps (Market Access Maps) du CEPII/CCI sur l'accès aux marchés. ${ }^{93}$

107. Afin de permettre l'analyse de la libéralisation des services fournis au travers d'une présence commerciale, une matrice des stocks bilatéraux de capitaux a été établie pour 2001. Les stocks bilatéraux d'IDE au niveau des secteurs du GTAP ont été estimés à partir de la nouvelle base de données de l'OCDE sur l'IDE, de l'annuaire de l'investissement dans le monde (World Investment Directory) publié par la CNUCED, des sources des autorités locales pour ce qui est de la Chine, de Hong Kong, Chine, de la Russie, de Singapour, du Chili, du Pérou et du Brésil, et de l'ANASE (2004) s'agissant de la Malaisie, des Philippines, de la Thaïlande et du Vietnam. Ces informations ont permis d'établir une base de données cohérente sur les stocks bilatéraux d'IDE par région et par secteur à l'aide de la méthodologie déjà utilisée pour la constitution des précédentes bases de données FTAP. ${ }^{94}$

108. L'étude ci-dessous s'appuie sur les informations tirées de la base de données GTAP-6, ainsi que sur la nouvelle base de données sur l'IDE utilisée pour les besoins du modèle FTAP, pour prendre en considération dans l'analyse 13 régions et 23 secteurs. Dix des treize régions sont constituées de pays de la zone OCDE. Sur les 23 secteurs, deux recouvrent des produits agroalimentaires, sept des produits manufacturés et onze des services. La correspondance entre les régions et les secteurs modélisés et leurs composantes dans la base de données GTAP-6 est indiquée aux tableaux A5.1 et A5.2. L'annexe 3 décrit la structure des échanges et des flux d'IDE entre la Chine et les pays de l'OCDE, qui est à la base des résultats des simulations examinés ci-dessous.

\section{Scénarios de simulation}

\section{Profils tarifaires de la Chine et impact de son accession à l'OMC}

109. Les données sur les droits de douane bilatéraux sont tirées de la base de données GTAP, version 6.1, qui est elle-même dérivée de la base de données MacMaps (Market Access Maps) du CEPII/CCI sur l'accès aux marchés, ${ }^{95}$ laquelle tient compte des droits bilatéraux ad valorem (NPF et préférentiels), des équivalents ad valorem des droits spécifiques (NPF et préférentiels), ainsi que des contingents tarifaires. ${ }^{96}$ L'équivalent ad valorem des mesures de protection appliquées ainsi obtenu constitue donc une mesure globale du degré de protection qui tient compte de manière exhaustive des préférences tarifaires en vigueur en 2001 et s'avère cohérente pour tous les flux commerciaux bilatéraux. Les données sur l'impact des engagements pris par la Chine à l'OMC en ce qui concerne les taux de droits appliqués sont issues d'une version connexe de la base de données GTAP comportant des données sur divers scénarios de réduction tarifaire tenant compte des engagements pris par la Chine à l'OMC. Les scénarios de réduction tarifaire envisagés ont été élaborés par le CEPII à partir de la base de données tarifaires MacMap au niveau des codes à 6 chiffres du SH (CEPII/CCI), ainsi que des droits de douane consolidés tirés des listes des tarifaires codifiées (LTC) de l'OMC.

110. Lors de l'accession à l'OMC, la Chine a accepté de consolider la totalité de ses droits à l'importation. Lorsque tous ses engagements en matière d'accès aux marchés auront été mis en œuvre, le

\footnotetext{
93 Cet ensemble de données est décrit en détail in A. Bouët, et al. (2002), Market Access for GTAP: A Bilateral Measure of Merchandise Trade Protection, GTAP Resource \#1045, disponible à l'adresse suivante (au 8 janvier 2005) : http://www.gtap.agecon.purdue.edu/resources/res_display.asp?RecordID=1045.

$94 \quad$ Voir Phamduc (2000).

95 Voir A. Bouët, et al. op. cit.

96

Ibid.
} 
niveau moyen des droits de douane consolidés de la Chine sera ramené à $15 \%$ pour les produits agricoles et à $8.9 \%$ pour les produits industriels $(\mathrm{OMC}, 2001)$. Les deux volets du tableau A5.3 présentent les droits de douane pondérés en fonction des échanges bilatéraux appliqués par la Chine au cours de l'année de référence 2001 et après la mise en œuvre des engagements pris à l'OMC, prévue pour 2004 pour la plupart d'entre eux, mais en tout état de cause en 2010 au plus tard (OMC, 2001). Les colonnes situées à l'extrême droite et les lignes figurant tout en bas de chacun des volets présentent respectivement les moyennes pondérées en fonction des échanges par catégorie de produit et par pays partenaire et la même information est présentée sous forme graphique aux diagrammes A5.1 et A5.2. Pour ce qui est de l'année de référence, la structure des droits de douane de la Chine indique que des niveaux élevés de protection sont appliqués dans l'absolu comme en termes relatifs dans les secteurs suivants : agriculture et pêche (droits de douane moyens pondérés en fonction des échanges égaux à $50 \%$ ), véhicules et pièces automobiles (38\%), textiles, habillement et cuir (19\%), produits alimentaires et boissons (18\%), substances et produits chimiques (13\%) et machines et équipements et autres produits manufacturés (environ 12\%). Les droits de douane moyens sont faibles dans le cas des importations de ressources naturelles, de pétrole et de charbon et relativement bas, puisqu'ils ne sont que de $7.5 \%$, dans celui des importations de produits métalliques.

111. Le degré d'agrégation des produits nécessaire pour procéder aux simulations EGC fait assurément obstacle à toute analyse détaillée de la structure des droits de douane, mais il n'en apparait pas moins très clairement que le profil tarifaire de la Chine observé en 2001 reflétait une politique délibérée de protection du secteur manufacturier, en particulier dans le cas des biens de consommation finale et des produits manufacturés à forte intensité de capital, des droits de douane relativement faibles étant toutefois imposés sur les intrants nécessaires au développement des secteurs d'exportation. Gaulier et al. (2005) soulignent que la politique tarifaire de la Chine n'est pas fondamentalement différente de celles appliquées au cours de leur histoire économique par les autres pays d'Asie orientale, qui ont assuré la protection des industries nationales au moyen de droits de douane relativement élevés et favorisé les exportations grâce à des exonérations de droits sur les intrants importés pour la production destinée à l'exportation. Plus généralement, il est probable que les droits dont il est fait état au tableau A5.3 surestiment quelque peu le degré de protection assuré par la Chine dans certains secteurs du fait des écarts très fréquemment observés entre les taux de droits nominaux et ceux effectivement perçus, en raison des larges exonérations de droits à l'importation associées aux échanges liés aux activités de transformation (Banque mondiale, 1994 ; Bach et al., 1996). Sur la base des données de 1997, Li et Zhai (2002) indiquent que ces écarts étaient particulièrement élevés dans le secteur textile et relativement faibles dans le cas des produits médicaux et automobiles, l'ampleur de ces écarts étant en règle générale d'autant plus grand que le secteur considéré était tourné vers l'exportation.

112. La mise en œuvre des engagements pris par la Chine à l'OMC dont il est tenu compte dans cet exercice de modélisation aboutit en moyenne à une réduction de $40 \%$ de la protection tarifaire. Des pourcentages de réduction tarifaire supérieurs à la moyenne sont escomptés dans les secteurs suivants : matériel électronique (réduction d'environ $81 \%$ des droits de douane moyens pondérés en fonction des échanges), agriculture et pêche (80\%), véhicules et pièces automobiles $(60 \%)$, textiles, habillement et cuir $(50 \%)$ et machines et équipements $(45 \%)$. Il est également clair que, du fait de variations dans la composition de leurs échanges avec la Chine ainsi que de la structure des concessions négociées, la mise en œuvre des engagements pris lors de l'accession à l'OMC n'aboutira pas à des concessions en matière d'accès au marché d'ampleur égale pour tous les pays membres de l'OCDE. Les réductions des droits de douane moyens pondérés en fonction des échanges seront supérieures à la moyenne pour le Mexique (73\%), les États-Unis (70\%), le Japon (54\%), l’UE-15 (51\%) et la Nouvelle-Zélande (45\%).

113. Les scénarios examinés ici tiennent non seulement compte d'un abaissement des droits à l'importation appliqués par la Chine à la suite de son accession à l'OMC mais aussi de la suppression par les États-Unis, l'UE-15 et le Canada des contingents d'importation appliqués dans le secteur des textiles, de l'habillement et du cuir en vertu de l'Accord sur les textiles et les vêtements (ATV). Conformément à la 
pratique actuelle et compte tenu de la disponibilité des données correspondantes, cette évolution est modélisée sous la forme d'une réduction des équivalents de taxes à l'exportation sur les livraisons chinoises de ces produits aux économies en question (Walmsley et Hertel, 2000, ainsi que Ianchovichina et Walmsley, 2003). ${ }^{97}$ Les équivalents de taxes ad valorem auxquels étaient soumises en 2001 les exportations du secteur des textiles, de l'habillement et du cuir à destination de l'UE-15, des États-Unis et du Canada ont été respectivement estimés à 18, 11 et 10\%.

\section{Engagements pris par la Chine à l'OMC dans le domaine des services}

Obstacles initiaux

114. Contrairement à la majorité des études existantes, le présent document procède à une simulation de la libéralisation des services sur la base des engagements effectivement pris par la Chine et non à partir de diverses hypothèses. Pour ce faire, l'analyse s'appuie sur une série de nouvelles estimations des obstacles aux échanges de services fondés sur des indices de restriction plus précis calculés séparément pour chaque mode de fourniture de services et tenant compte de certains autres aspects relatifs aux réglementations, tels que le degré de transparence des réglementations en vigueur ou le statut des organismes de réglementation nationaux. L'OCDE (2005j) décrit en détail la méthode d'estimation de ces équivalents de taxes. Les équivalents de taxes des obstacles auxquels se heurtent la Chine et les pays de l'OCDE étudiés dans le domaine des télécommunications, de la banque, de l'assurance, de la distribution et des services professionnels (ingénierie) sur lesquels se fonde cette analyse sont présentés au tableau A5.4. Il importe de noter que ces équivalents de taxes sont estimés au moyen de techniques statistiques et comportent donc inévitablement une part d'incertitude.

115. Les résultats des simulations ne sont pas seulement fonction de la qualité des estimations des obstacles aux échanges mais dépendent également de la manière dont leur suppression est prise en compte dans le modèle. La méthode de modélisation de la protection exerce une forte influence sur les résultats globaux de la libéralisation, que ce soit au travers des effets sur les revenus (lorsque les obstacles sont modélisés sous la forme de mesures à l'origine de rentes) ou des effets sur l'efficience allocative (lorsque les obstacles sont modélisés sous la forme de facteurs d'augmentation des coûts). Dans toutes les études, le choix du mode de modélisation de ces obstacles est dans une certaine mesure arbitraire et il est souvent déterminé par la méthode retenue pour en estimer l'ampleur. Les obstacles aux échanges de services paraissent importants et devraient donc exercer une notable influence sur les résultats des simulations. Compte tenu des incertitudes quant à l'interprétation de la nature des obstacles aux échanges de services, ${ }^{98}$ il n'a pas été envisagé, lors de leur intégration dans la base de données, d'établir de distinction entre ceux à l'origine de rentes et ceux constituant des facteurs d'augmentation des coûts, vu les difficultés à faire la différence entre les uns et les autres. Les obstacles aux échanges de services ont été introduits dans la base de données sous la forme de taxes sur la production et de taxes à l'importation. ${ }^{99}$

116. Les équivalents de taxes dans le secteur des services présentés au tableau A5.4 ont été introduits dans le modèle GTAP à l'aide de l'option "Altertax", qui donne la possibilité de modifier les divers taux de taxes inclus dans la base de données. Cette procédure est conçue pour permettre d'ajouter des informations sur les variables de décision aux données déjà groupées dans le modèle (Malcolm, 1998). Les taxes ont donc pu être prises en considération, tout en sauvegardant la cohérence interne de la base de données et en limitant au minimum les répercussions de la modification des droits de douane sur la valeur des biens et sur les flux financiers. La base de données actualisée contenant les estimations des taxes sur les services constitue le fondement sur lequel s'appuient les étapes suivantes.

\footnotetext{
$97 \quad$ Dans une telle situation, les rentes découlant des contingents sont réputées revenir aux exportateurs.

98 Voir OCDE (2005j) pour un examen approfondi de cette question.

99 Tous les obstacles ont été explicitement introduits sous la forme d"“équivalents de taxes".
} 


\section{Engagements pris à l'OMC}

117. Ces résultats sont conformes aux constatations d'ensemble, qui montrent qu'avant la mise en œuvre progressive des engagements de libéralisation inscrits sur sa liste de l'AGCS, le nombre de secteurs où un plein accès était garanti était plus faible en Chine que dans toutes les autres catégories de pays (développés, en développement, et candidats à l'accession). ${ }^{100}$ Cet état de choses concorde avec le niveau de l'indice de restriction des échanges (IRE) et avec les équivalents de taxes tels qu'ils ont été calculés par l'OCDE (2005j). Comme le fait remarquer Mattoo (2003), le tableau pourrait être radicalement différent après la mise en œuvre des engagements de libéralisation. Dans l'ensemble, les engagements pris dans le domaine de l'accès aux marchés sont d'une ampleur et d'une étendue bien plus grandes que les concessions offertes lors du Cycle d'Uruguay par n'importe quelle autre catégorie de pays (y compris ceux à revenu élevé). Par ailleurs, les engagements de la Chine en matière de traitement national sont de plus grande ampleur et de plus grande étendue que ceux de toutes les autres catégories de pays.

118. Les scénarios de simulation correspondant à la mise en œuvre des engagements de la Chine dans les cinq secteurs de services ont été définis en attribuant une note aux différentes composantes de l'indice de restriction des échanges sur la base des engagements pris par la Chine dans le cadre de l'AGCS, compte tenu (i) de l'état de la situation en 2001 (date de l'accession) et (ii) de l'état de la situation en 2008 (date à laquelle tous les engagements de libéralisation auront été mis en œuvre). L'analyse sectorielle menée à l'annexe 1 décrit les modifications apportées aux politiques mises en auvre en Chine, alors que les variations enregistrées par les estimations des IRE sont présentées sous forme graphique aux diagrammes A1.11 à A1.6. Les réductions des équivalents de taxes qui devraient s'ensuivre dans le domaine des services du fait de la mise en œuvre des engagements pris à l'OMC dans ces cinq secteurs de services apparaissent au tableau A5.4.

\section{Examen des résultats des simulations}

\section{Effets à l'échelle mondiale}

119. Les tableaux 14,15 et 16 ci-dessous présentent les pourcentages de variation du revenu réel et du degré de bien-être économique (telles que mesurées par la variation équivalente), de même que les gains de bien-être par habitant résultant de la mise en œuvre par la Chine des engagements pris à l'OMC ainsi que de la libéralisation complète des échanges pour tous les biens et certains secteurs de services (banque, assurance, communication, autres services aux entreprises et distribution). La variation équivalente du revenu correspond à l'équivalent monétaire de la variation de l'utilité entraînée par la variation des prix. Autrement dit, elle ne signifie pas nécessairement que le revenu du bénéficiaire enregistrera effectivement une variation d'une telle ampleur, car elle constitue plutôt une mesure de la variation de l'utilité. Il s'ensuit qu'en principe les deux mesures de la performance économique présentées dans ces deux tableaux peuvent parfois être différentes, y compris pour ce qui est de leur signe.

120. Les tableaux montrent que la mise en œuvre par la Chine des engagements pris à l'OMC pour ce qui est des biens et de cinq secteurs de services entraînent une augmentation de son revenu réel et de son bien-être par habitant qui est estimée à près de $2 \%$, alors qu'une libéralisation complète des échanges dans ces secteurs devrait accroître d'environ 3\% son revenu réel et son bien-être par habitant. Du point de vue des effets sur le bien-être, la Chine devrait enregistrer un gain d'environ 17.6 milliards USD à la suite de la mise en œuvre de ses engagements à l'OMC dans les secteurs en question, dont environ 13.7 milliards USD dans l'agriculture et l'industrie manufacturière ${ }^{101}$ et 2.8 autres milliards USD dans les secteurs de services concernés. Une totale libéralisation des échanges offrirait à la Chine des gains de bien-être

\footnotetext{
$100 \quad$ Voir Mattoo (2003).

101 La suppression des contingents imposés dans le cadre de l'ATV aux exportations chinoises vers l'UE15, les États-Unis et le Canada étant également prise en compte.
} 
d'environ 28 milliards USD, dont 15.9 milliards USD proviendraient de la libéralisation des échanges de marchandises et 11.9 milliards USD de celle du commerce de services. Ces résultats sont conformes à la plupart des conclusions tirées de l'examen des études sur la question.

121. Le monde dans son ensemble devrait certes bénéficier, d'après les projections, de gains de bien-être de près de 30 milliards USD dans le premier scénario et de 48.2 milliards USD dans le second, mais les effets sur chacun des pays de l'OCDE devraient néanmoins être assez limités dans l'un et l'autre cas.

Tableau 14. Impact sur le revenu réel et sur le bien-être de la mise en œuvre par la Chine de ses engagements à l'OMC pour la totalité des biens et certains secteurs de services

\begin{tabular}{|c|c|c|c|c|c|c|}
\hline & \multicolumn{2}{|c|}{ Real Income } & \multirow[b]{2}{*}{ Services \% } & \multirow[b]{2}{*}{ Total USD m } & \multicolumn{2}{|c|}{ Equivalent variation (EV) } \\
\hline & Total \% & Goods \% & & & Goods USD m & Services USD m \\
\hline Australia & -0.08 & -0.08 & 0.01 & -248 & -227 & 20 \\
\hline New Zealand & 0.03 & 0.04 & -0.01 & 13 & 14 & -2 \\
\hline China & 1.95 & 1.51 & 0.35 & 17695 & 13761 & 2821 \\
\hline Hong Kong, China & 0.81 & 0.67 & 0.14 & 1351 & 1072 & 278 \\
\hline Russia & -0.06 & -0.05 & -0.01 & -177 & -142 & -27 \\
\hline Japan & 0.07 & 0.06 & 0.00 & 2661 & 2451 & 63 \\
\hline Korea & 0.19 & 0.20 & 0.01 & 617 & 647 & 26 \\
\hline Canada & -0.05 & -0.04 & 0.00 & -280 & -232 & 3 \\
\hline United States & 0.05 & 0.04 & 0.01 & 4717 & 4272 & 615 \\
\hline Mexico & -0.10 & -0.09 & 0.00 & -192 & -164 & 19 \\
\hline EU15 & 0.08 & 0.07 & 0.01 & 4655 & 3899 & 389 \\
\hline Rest of Europe & 0.01 & 0.01 & 0.00 & -63 & -36 & -1 \\
\hline $\begin{array}{l}\text { Rest of the World } \\
\text { Total }\end{array}$ & -0.05 & -0.04 & 0.01 & $\begin{array}{l}-1175 \\
29575\end{array}$ & $\begin{array}{l}-1149 \\
24167\end{array}$ & $\begin{array}{c}282 \\
4486\end{array}$ \\
\hline
\end{tabular}

Source : projections du modèle FTAP

Tableau 15. Impact sur le revenu réel et sur le bien-être de l'élimination par la Chine des obstacles aux échanges pour la totalité des biens et certains secteurs de services

\begin{tabular}{|c|c|c|c|c|c|c|}
\hline & \multicolumn{2}{|c|}{ Real Income } & \multirow[b]{2}{*}{ Services \% } & \multirow[b]{2}{*}{ Total USD m } & \multicolumn{2}{|c|}{ Equivalent variation (EV) } \\
\hline & Total $\%$ & Goods \% & & & Goods USD m & Services USD m \\
\hline Australia & -0.04 & -0.01 & -0.04 & -131 & -45 & -99 \\
\hline New Zealand & 0.19 & 0.16 & -0.01 & 83 & 69 & -3 \\
\hline China & 2.99 & 1.71 & 1.34 & 27904 & 15952 & 11973 \\
\hline Hong Kong, China & 1.22 & 1.00 & 0.37 & 2806 & 2009 & 719 \\
\hline Russia & 0.19 & 0.17 & -0.07 & 509 & 479 & -191 \\
\hline Japan & 0.20 & 0.15 & 0.04 & 7852 & 6280 & 1089 \\
\hline Korea & 0.57 & 0.54 & -0.01 & 2270 & 2179 & -63 \\
\hline Canada & -0.05 & -0.01 & -0.07 & -306 & -78 & -377 \\
\hline United States & 0.05 & 0.05 & 0.00 & 5188 & 4614 & 575 \\
\hline Mexico & -0.10 & -0.08 & -0.05 & -29 & -30 & -93 \\
\hline EU15 & -0.02 & 0.01 & 0.00 & -2272 & -116 & -96 \\
\hline Rest of Europe & 0.10 & 0.08 & 0.01 & 712 & 522 & 66 \\
\hline Rest of the World & 0.07 & 0.05 & 0.00 & 3680 & 2700 & 230 \\
\hline Total & & & & 48267 & 34535 & 13730 \\
\hline
\end{tabular}


Tableau 16. Gains de bien-être par habitant : mise en œuvre des engagements pris à l'OMC et libéralisation complète des échanges

\begin{tabular}{lccc|ccc}
\hline & \multicolumn{3}{c|}{ WTO accession } & \multicolumn{3}{c}{ Full liberalisation } \\
& Total \% & Goods \% & Services \% & Total \% & Goods \% & Services \% \\
\hline Australia & -0.08 & -0.07 & 0.01 & -0.04 & -0.01 & -0.03 \\
New Zealand & 0.03 & 0.04 & -0.01 & 0.20 & 0.16 & -0.01 \\
China & 1.90 & 1.48 & 0.30 & 2.91 & 1.67 & 1.29 \\
Hong Kong- China & 0.84 & 0.67 & 0.17 & 1.41 & 1.01 & 0.45 \\
Russia & -0.07 & -0.06 & -0.01 & 0.17 & 0.16 & -0.08 \\
Japan & 0.08 & 0.07 & 0.00 & 0.21 & 0.17 & 0.03 \\
Korea & 0.20 & 0.21 & 0.01 & 0.59 & 0.57 & -0.02 \\
Canada & -0.05 & -0.04 & 0.00 & -0.05 & -0.01 & -0.07 \\
United States & 0.05 & 0.05 & 0.01 & 0.06 & 0.05 & 0.01 \\
Mexico & -0.04 & -0.03 & 0.00 & -0.01 & -0.01 & -0.02 \\
EU15 & 0.07 & 0.06 & 0.01 & -0.04 & 0.00 & 0.00 \\
Rest of Europe & -0.01 & 0.00 & 0.00 & 0.08 & 0.06 & 0.01 \\
Rest of the World & -0.03 & -0.03 & 0.01 & 0.09 & 0.07 & 0.01 \\
\hline Source : projections du modèle FTAP
\end{tabular}

122. La prochaine section offre une étude détaillée au niveau sectoriel comme à celui de l'économie dans son ensemble des différents effets sur le bien-être qu'une libéralisation des échanges de biens et services entraînerait pour la Chine aussi bien que pour les pays et régions de l'OCDE objet de l'analyse, et elle présente également la décomposition des facteurs qui y contribuent. Les principaux facteurs qui déterminent ces effets sur le bien-être sont les suivants :

1. Effets sur l'efficience allocative, qui mesurent les variations dans l'allocation des ressources à la suite des modifications apportées aux politiques mises en œuvre par les pouvoirs publics,

2. Effets sur le revenu, qui résultent de l'expansion ou de la contraction des stocks de capitaux situés dans une région donnée,

3. Effets sur la diversité des produits, qui recouvrent les avantages qu'une plus grande diversité des biens ou services d'une certaine catégorie pourrait procurer aux consommateurs,

4. Effets sur les termes de l'échange, qui mesurent les variations du prix relatif des exportations et des importations d'un pays ou d'une région,

5. Effets liés à l'IDE :

- Variations des rentes normales tirées de l'IDE : rentes normales versées aux détenteurs de capitaux étrangers par le pays ou la région d'accueil et rentes versées aux détenteurs de capitaux étrangers dans le pays ou la région d'origine,

- Variations des rentes découlant des obstacles aux échanges tirées par les détenteurs de capitaux étrangers et les filiales d'entreprises étrangères du pays ou de la région d'origine : montant des rentes découlant des obstacles aux échanges versées par le pays ou la région d'accueil aux détenteurs de capitaux étrangers et aux filiales d'entreprises étrangères diminué du montant des rentes découlant des obstacles aux échanges versées aux propriétaires de capitaux étrangers et aux filiales d'entreprises étrangères dans leur pays ou leur région d'origine,

6. Effets sur la dette extérieure :

- Revenus perçus ou versés par un pays ou une région au titre de ses créances ou de ses dettes extérieures, et 
- Montant des intérêts perçus ou versés par un pays ou une région au titre de ses créances ou de ses dettes extérieures.

123. Les facteurs générateurs de revenus (tels que les effets sur l'efficience allocative, les dotations nettes en capital et la diversité des produits) sont ceux dont l'impact est le plus important pour ce qui est du monde dans son ensemble, mais ils doivent être analysés en parallèle avec les facteurs de redistribution des revenus (tels que les effets sur les revenus tirés de l'IDE car dans leur cas les gains enregistrés par une région donnée peuvent impliquer des pertes pour une autre. La prochaine section examine ces résultats, les tableaux présentant les résultats détaillés étant regroupés à l'annexe 5.

\section{Libéralisation des échanges de biens}

124. La mise en œuvre par la Chine des engagements pris à l'OMC devrait procurer au monde dans son ensemble des gains de bien-être d'un montant d'environ 24 milliards USD par an, dont près de 14 milliards USD pour la Chine elle-même (tableau 14). Ces 14 milliards USD dont devrait bénéficier la Chine correspondent à un gain annuel par habitant non négligeable, puisqu'il s'élève à $1.48 \%$ (tableau 16). Ces gains résultent pour l'essentiel d'une utilisation plus efficiente des ressources en Chine. En fait, d'après les estimations, ces "gains d'efficience allocative" devraient s'élever à environ 21 milliards USD mais sont contrebalancés par les pertes liées aux variations des termes de l'échange ainsi que par les effets négatifs associés aux investissements directs étrangers de la Chine et dans une moindre mesure au service de sa dette extérieure.

125. Les importants gains d'efficience allocative obtenus en Chine résultent d'un manifeste redéploiement des ressources au détriment de certains secteurs très protégés tels que l'agriculture et la pêche, les véhicules et pièces automobiles, les substances et produits chimiques et les machines et équipements et au profit d'autres secteurs relativement moins protégés mais aussi en cours d'ouverture, tels que les textiles, l'habillement et le cuir, le matériel électronique, ou encore les produits alimentaires et les boissons. Cette description du mode de réallocation des ressources vaut aussi bien pour les producteurs locaux chinois que pour les producteurs étrangers implantés en Chine.

126. Cette évolution en matière d'allocation des ressources est liée aux variations très spectaculaires des importations chinoises, la plupart des secteurs enregistrant d'importantes augmentations des volumes importés. D'après les estimations, les progressions les plus marquées des importations chinoises ont été enregistrées dans l'agriculture et la pêche (variation de 116\% des volumes importés), les véhicules et pièces automobiles $(57 \%)$ et les textiles, l'habillement et le cuir (44\%), et elle est supérieure à $15 \%$ pour ce qui est des produits alimentaires et des boissons, des machines et équipements, ainsi que du matériel électronique. Une partie de cette demande accrue d'importations est certes imputable à la consommation finale, mais l'essentiel correspond à une augmentation de la demande d'intrants intermédiaires de la part des entreprises locales et étrangères qui produisent en Chine. En effet, malgré des ordres de grandeur un peu plus modestes, l'expansion des exportations présente un profil très semblable : textiles, habillement et cuir $(36 \%)$, agriculture et pêche $(24 \%)$, véhicules et pièces automobiles $(21 \%)$, produits alimentaires et boissons (17\%) et matériel électronique (15\%). L'interaction entre les variations des importations et des exportations illustre l'impact de l'ouverture des marchés sur les coûts des intrants intermédiaires des producteurs localisés en Chine et par voie de conséquence sur leur compétitivité à l'exportation. Dans le même temps, elle donne à penser que l'impact sur les économies de l'OCDE sera limité puisqu'elles pourront certes exporter plus librement vers le marché chinois mais seront confrontées à une concurrence accrue de la part des producteurs localisés en Chine.

127. Cette importante mutation structurelle s'accompagne de variations des stocks d'entrées d'IDE (tableau A5.9). Dans le modèle FTAP qui tient compte de la présence étrangère de producteurs de la zone OCDE sur le marché chinois, l'impact exercé sur les pays de l'OCDE par le meilleur accès aux marchés 
dépend également de l'ampleur de leur présence au sein de l'économie chinoise ainsi que de l'objectif de cette présence. La présence à l'étranger est un moyen de surmonter les importants obstacles initiaux à l'accès aux marchés, aussi la libéralisation pourrait-elle aboutir à un désinvestissement s'il devient plus efficient d'approvisionner le marché chinois depuis l'extérieur. Si par contre la présence à l'étranger demeure un mode plus efficient d'approvisionnement du marché chinois (ou du moins d'approvisionnement des autres marchés d'exportation à partir de la Chine), la libéralisation de la Chine pourrait accroître l'ampleur de la présence étrangère en Chine et être profitable aux investisseurs, qui bénéficieraient d'intrants intermédiaires encore meilleur marché pour assurer leur production. Les résultats effectifs de la simulation font apparaître que, tous investisseurs étrangers confondus, la plus forte augmentation du stock d'IDE intervient dans l'agriculture et la pêche $(0.4 \%)$ alors que la plus forte diminution a lieu dans les textiles, l'habillement et le cuir $(-0.5 \%)$. Les variations sont certes plus faibles dans les autres secteurs manufacturiers, mais tous enregistrent des augmentations des stocks d'IDE (voir le tableau A5.9).

128. Ces importantes variations du volume des échanges chinois ont également une forte incidence sur les prix mondiaux des produits échangés - une des voies par lesquelles la libéralisation de la Chine influe sur la structure de la production et de l'emploi dans l'ensemble de l'économie mondiale. Il importe ici de noter que l'indice des prix mondiaux à l'exportation dans l'agriculture et la pêche augmente au taux non négligeable de $3 \%$ alors que les prix baissent dans la plupart des secteurs manufacturiers. L'indice des prix mondiaux diminue de pas moins de $20 \%$ en ce qui concerne les textiles, 1 'habillement et le cuir, et de $3.4 \%$ pour ce qui est du matériel électronique. Dans d'autres secteurs manufacturiers, les augmentations de prix sont moins marquées. Les résultats suggèrent que l'intégration de la Chine dans l'économie mondiale pourrait bénéficier aux producteurs agricoles au travers d'une augmentation de la demande, alors que les fournisseurs de produits manufacturés pourraient être confrontés à une baisse des prix à l'exportation du fait de la concurrence des producteurs chinois.

129. Conformément aux attentes, les gains considérables associés à une meilleure allocation des ressources productives au sein de l'économie chinoise sont contrebalancés par une dégradation assez sensible des termes de l'échange. Il n'est guère surprenant que la Chine subisse une perte du fait de l'évolution des termes de l'échange, puisque le scénario envisagé se limite dans une large mesure à une diminution des obstacles à l'importation en Chine et implique donc un fort essor de la demande chinoise d'importations qui aboutit à une hausse des prix des importations chinoises. En outre, les prix des exportations chinoises baissent du fait de la réduction des coûts de production ainsi que de la nécessité d'exporter davantage pour financer la demande accrue d'importations. Ces deux phénomènes ont un effet global négatif sur les termes de l'échange de la Chine égal à $-1.8 \%$, ce qui correspond à la différence en points de pourcentage entre la hausse du prix global des importations et le prix global des exportations. La taille importante de l'économie chinoise contribue inévitablement à l'ampleur des effets négatifs sur les termes de l'échange. Par contre, les partenaires commerciaux de la Chine bénéficient en règle générale d'améliorations de leurs termes de l'échange.

130. Un autre facteur atténue l'ampleur des gains tirés par la Chine de sa propre ouverture, et il tient aux pertes subies par le pays sur ses investissements directs à l'étranger (tableau A5.5). Ces pertes sont associées à l'impact que la libéralisation de la Chine exerce sur les taux de rendement des investissements directs à l'extérieur du pays. Dans le même temps, la mise en œuvre par la Chine des engagements pris à l'OMC lui permettrait de bénéficier d'une plus grande dotation en capital et d'une plus grande diversité de produits, facteurs contribuant tous deux de manière positive aux gains de bien-être en faveur du pays.

131. L'impact de la mise en œuvre par la Chine de ses engagements tarifaires et la suppression des contingents ATV ont une incidence assez limitée sur les pays membres de l'OCDE (tableau A5.5). En termes absolus, les principaux gagnants sont les États-Unis et 1'UE-15, qui tirent respectivement des gains annuels d'environ 4.2 et 3.9 milliards USD. Parmi les autres pays membres de l'OCDE qui enregistrent un 
gain figurent les partenaires commerciaux proches de la Chine, à savoir le Japon et la Corée. L'Australie, le Canada et le Mexique devraient quant à eux subir des pertes de faible ampleur. En termes de gains de bien-être par habitant (voir le tableau 16), le principal gagnant est la Corée, avec $0.2 \%$ d'augmentation annuelle du bien-être par habitant, suivie par le Japon (0.07\%), l'UE-15 (0.06\%) et les États-Unis (0.05\%). Tous les pays de l'OCDE gagnants profitent d'une amélioration de l'efficience allocative, de considérables effets favorables sur leurs termes de l'échange, ainsi que d'une augmentation des revenus tirés de l'IDE dans le secteur des services en Chine.

132. Trois économies de l'OCDE, l'Australie, le Canada et le Mexique devraient connaître un recul du bien-être par habitant de $0.07,0.04$ et $0.03 \%$ respectivement. Ces pertes découlent principalement de la dégradation des termes de l'échange (Mexique et Australie) et de l'alourdissement du service de la dette extérieure du fait de la hausse des taux d'intérêt réels mondiaux (Australie, Canada et Mexique). Les effets défavorables sur les termes de l'échange sont imputables aux pertes de parts du marché chinois subies du fait de l'impact inégal des engagements pris par la Chine à l'OMC sur les prix après impôt des importations en provenance des différents pays de l'OCDE. Ainsi, l'Australie, qui exporte vers la Chine une part non négligeable de sa production agricole (tableau A3.1), ne paraît pas bénéficier d'aussi importantes réductions des tarifs agricoles que les États-Unis et le Reste du monde (tableau A5.3), et doit donc répondre à l'abaissement des droits de douane par une diminution de ses prix à l'exportation (d'où l'effet négatif sur ses termes de l'échange), ou s'exposer si elle s'en abstient à perdre des parts de marché. Le Mexique souffre également d'une dégradation de ses termes de l'échange mais la perte totale qu'il subit est principalement due à l'alourdissement du service de sa dette extérieure.

133. Le faible impact de l'ouverture de la Chine sur les pays de l'OCDE est en grande partie conforme aux études existantes réalisées au moyen de modèles EGC et, plus fondamentalement, aux données commerciales sous-jacentes. En effet, la vaste analyse de la structure des échanges entre la Chine et les pays de l'OCDE qui est présentée à l'annexe 3 donne à penser que la dépendance à l'égard de la Chine en tant que marché de destination des exportations était assez limitée en 2001, hormis pour les économies de la zone OCDE situées en Asie et en Océanie. La part du PIB du pays d'origine que représentent les exportations vers la Chine est particulièrement élevée en Corée (6.7\%), en Nouvelle-Zélande $(2.2 \%)$, en Australie (1.5\%) et au Japon (1.2\%), et elle tend là encore à être plus faible au Canada $(0.7 \%)$, aux ÉtatsUnis $(0.3 \%)$, dans 1'UE-15 (0.6\%) et au Mexique (0.2\%). À titre de comparaison, les exportations des États-Unis vers l'UE-15 comptaient en 2001 pour $29 \%$ de l'ensemble de leurs exportations et pour $2.7 \%$ de leur PIB, alors que les exportations de l'UE-15 vers les États-Unis représentaient $11 \%$ de l'ensemble de ses exportations $^{102}$ et aux alentours de $3.7 \%$ de son PIB.

134. Le scénario fondé sur l'hypothèse d'une suppression par la Chine de la totalité de ses droits à l'importation aboutit à un accroissement des gains pour l'ensemble du monde faisant passer ceux-ci de 24 milliards USD à 34 milliards USD, soit une augmentation de un tiers (tableau A5.6). Cela paraît en tout premier lieu indiquer que les engagements pris par la Chine à l'OMC en matière de droits de douane sont déjà très ambitieux et sont à l'origine de l'essentiel des gains que l'on peut attendre des réformes dans ce domaine particulier de la politique commerciale chinoise. Chose intéressante, le scénario d'une libéralisation complète réduit légèrement les gains de bien-être globaux tirés par la Chine : des gains potentiels pourraient certes découler d'une amélioration de l'efficience allocative imputable à la libéralisation plus poussée assurée par la Chine, mais les effets négatifs sur les termes de l'échange et sur la dette extérieure s'accroissent plus que proportionnellement et maintiennent les gains de bien-être globaux à un niveau comparable aux résultats découlant de l'accession de l'OMC.

135. Le résultat qui s'ensuit pour la Chine contraste avec celui observé pour la plupart des pays de 1'OCDE qui, mis à part l'UE-15, tendent à gagner davantage (ou à moins perdre) tant en termes absolus

102 Y compris les échanges internes à l'UE-15. 
que par habitant dans ce scénario de libéralisation plus ambitieux. La Corée conserve la première place sur la liste des gagnants mais ses gains sont encore plus importants et atteignent $0.57 \%$ par habitant. Viennent ensuite le Japon, avec des gains de bien-être par habitant $0.17 \%$, puis la Nouvelle-Zélande $(0.16 \%)$ et les États-Unis (0.05\%). L'Australie, le Canada et le Mexique enregistrent quant à eux des pertes un peu moindres, alors que l'UE-15 ne devrait plus bénéficier de gains importants mais supporter de légères pertes. Ce dernier effet est lié au fait que dans le scénario d'une complète libéralisation l'UE-15 devient vendeur net d'actifs financiers, ce qui implique une sensible augmentation du montant des intérêts à acquitter, par suite d'une hausse des taux d'intérêt. L'UE-15 enregistre en outre des pertes du fait des variations du prix de ses actifs d'IDE. L'une et l'autre de ces caractéristiques sont propres au modèle FTAP et devraient faire l'objet d'une analyse plus approfondie dans le cadre des travaux quantitatifs futurs.

136. Le scénario d'une complète libéralisation est plus favorable pour la Russie qui devient un important gagnant net (augmentation de $0.16 \%$ du bien-être par habitant) alors qu'elle était un perdant net dans le scénario de l'accession à l'OMC. Les effets défavorables sur les termes de l'échange et sur l'efficience allocative observés en Russie dans le scénario de l'accession de la Chine à l'OMC sont dus aux pertes de parts du marché chinois subies du fait que les engagements pris par la Chine à l'OMC ont un impact inégal sur les prix après impôt des importations en provenance de ses différents partenaires commerciaux. Il était assez prévisible qu'il en soit ainsi, compte tenu de la situation défavorisée dans laquelle s'est trouvée la Russie lors de la négociation des conditions d'accession de la Chine à l'OMC du fait de son statut de non membre, ainsi que de l'érosion de l'accès préférentiel au marché chinois dont bénéficiait la Russie avant 2001. Seule une libéralisation allant au-delà des engagements pris par la Chine à l'OMC créerait des possibilités d'accès aux marchés au bénéfice de la Russie.

\section{Libéralisation des services}

137. D'après les estimations, la mise en œuvre des engagements pris à l'OMC dans les secteurs de services retenus devrait générer des avantages de près de 3 milliards USD au profit de la Chine (voir le tableau A5.7), alors qu'une totale libéralisation de ces secteurs serait à l'origine de gains plus de trois fois supérieurs (voir le tableau A5.8). Cela indique que, malgré des engagements de grande ampleur dans le domaine des services, d'importants obstacles aux échanges de services subsistent.

138. Comme dans le cas de la libéralisation des échanges de biens, les effets pour la Chine d'une libéralisation des services consistent principalement en des améliorations de l'efficience allocative et en une dégradation des termes de l'échange. Conformément aux attentes, les gains d'efficience allocative sont très importants (compte tenu des obstacles initiaux élevés). Les améliorations de l'efficience allocative découlent des variations du volume de production de l'ensemble des secteurs et sont principalement déterminés par les flux de capitaux des autres pays ou régions vers la Chine dans les cinq secteurs de services analysés (voir le tableau A5.10). Compte tenu de l'ampleur initiale relativement grande des obstacles aux échanges de services réduits ou supprimés, la Chine attire d'importantes quantités de capitaux étrangers dans la distribution, l'assurance et les autres services aux entreprises. La dotation totale en capital s'en trouve élargie, de sorte que l'expansion des cinq secteurs de services ne s'accompagne pas de réductions des volumes de production dans les autres secteurs (voir le tableau A5.10, qui met en évidence l'absence de réallocation sectorielle des ressources). Cette réallocation au profit de la Chine des capitaux des régions de l'OCDE et du Reste du monde (qui exerce un effet positif sur la dotation en capital du pays) a en outre une incidence sur la diversité des produits en Chine, puisqu'elle est à l'origine d'une augmentation de la production associée à une plus grande diversité de l'offre émanant d'un nombre accru d'entreprises, d'où un plus grand choix pour les consommateurs. Les effets sur la diversité des produits renforcent par conséquent les gains tirés de la réallocation des capitaux.

139. Contrastant avec les gains d'efficience allocative, les effets sur les termes de l'échange sont négatifs pour la Chine. Cette dégradation des termes de l'échange est conforme aux attentes et à l'examen de la 
libéralisation des échanges de biens effectué ici : la réduction ou la suppression des obstacles relativement élevés aux échanges de services existant en Chine entraîne une baisse du prix relatif des exportations par rapport à celui des importations.

140. Quant aux effets liés à l'IDE, il importe de noter que la Chine subit des pertes sur ses investissements directs à l'étranger. La contribution des variations des stocks d'investissements directs à l'étranger au bien-être de la Chine est négative mais modeste, car la contribution positive des variations des capitaux étrangers investis en Chine (déterminées par la réduction des obstacles aux échanges de services selon le mode 3) est compensée par les pertes sur les capitaux chinois à l'étranger. La contribution au bienêtre en Chine des variations du taux de rendement est de même négative, du fait des évolutions à la baisse enregistrées à l'étranger.

141. Du point de vue des rentes générées par les obstacles aux échanges de services (contributions au revenu des variations de la rente découlant des obstacles aux services à mesure que ces obstacles sont éliminés), les rentes nettes tirées de l'IDE (égales aux rentes découlant des obstacles à la production) fluent des régions de l'OCDE ayant procédé aux investissements vers la Chine (qui en est le pays bénéficiaire). Les rentes nettes tirées de l'IDE représentent une importante source de gains pour la Chine et de pertes pour Hong Kong, Chine, et le Reste du monde. L'hypothèse concernant l'identité des bénéficiaires de ces rentes générées par les obstacles aux échanges est essentielle pour déterminer l'impact global sur le bienêtre de la libéralisation des services. Le modèle FTAP part du postulat que, pour ce qui est des entreprises étrangères, les rentes générées par les obstacles aux échanges bénéficient principalement au pays ou à la région d'origine des capitaux, seule une contribution à l'impôt sur le revenu des sociétés étant versée au pays ou à la région où est située l'entreprise. Aussi, conformément à cette hypothèse, les économies et les régions qui investissent en Chine enregistrent-elle des pertes.

142. D'après les estimations, l'impact sur les pays de l'OCDE est limité dans l'un et l'autre scénarios de libéralisation des services. L'effet sur l'efficience allocative tend à être très modeste (négatif pour Hong Kong, Chine, la Russie, le Japon et le Reste de l'Europe, et positif pour tous les autres pays et régions de l'OCDE), selon l'ampleur de la variation de leur stock de capital (qui est en général assez réduite, du fait des faibles parts initiales des flux d'investissement étranger à destination de la Chine). L'effet sur la dotation nette en capital est négatif dans la plupart des pays et régions de l'OCDE du fait des sorties accrues de capitaux à la suite des variations (à la baisse) des taux de rendement du capital dans ces pays et régions. Les effets sur la diversité des produits tendent de même à être assez faibles et négatifs dans les pays de l'OCDE. La plupart des pays de l'OCDE connaissent par contre de légères améliorations de leurs termes de l'échange (sauf le Canada, le Mexique et la Russie, qui enregistrent une dégradation des termes de l'échange).

143. Quant aux contributions liées à l'IDE, il importe de noter qu'elles occupent une place prépondérante dans les (modestes) effets sur le bien-être de la plupart des pays de l'OCDE. La Chine subira par ailleurs des pertes sur ses investissements directs à l'étranger, tandis que la plupart des pays de l'OCDE tireront des revenus accrus de leurs IDE en Chine. Les avantages générés par les revenus accrus tirés de l'IDE par les pays de l'OCDE s'expliquent principalement par le fait que tous les pays étaient initialement fournisseurs nets d'IDE dans les services en Chine. Par suite de la levée des obstacles à l'entrée, les pays de l'OCDE accroissent leur IDE en Chine et bénéficient d'une augmentation de la rémunération de leur IDE (taux accrus de rendement du capital en Chine). Dans un petit nombre de pays ou régions de l'OCDE (Nouvelle-Zélande et Reste de l'Europe) les gains tirés de l'accroissement de l'IDE en Chine ne compensent pas les pertes subies sur l'IDE dans les autres pays ou régions.

144. En résumé, la Chine tire essentiellement avantage des effets nets sur l'efficience allocative, sur la diversité des produits et sur les mouvements de capitaux, alors que les pays et régions de l'OCDE bénéficient d'améliorations de leurs termes de l'échange et d'augmentations des volumes nets d'IDE. 
Initialement plus protégés, la Russie, la Corée et le Mexique enregistrent des pertes du fait de l'évolution de leurs termes de l'échange. La Chine subit de même une dégradation des termes de l'échange mais les effets globaux sur le bien-être demeurent positifs.

\section{Conclusions}

145. Le présent document examine l'émergence de la Chine en tant qu'acteur mondial sur les marchés internationaux au cours des toutes dernières décennies. Il offre une vue d'ensemble du cadre de la politique commerciale de la Chine après l'ouverture de ses marchés et son adhésion à l'OMC. Il décrit la part croissante prise par la Chine dans les échanges mondiaux, notant qu'elle est désormais la troisième puissance commerciale après les États-Unis et l'Allemagne. Ses liens commerciaux avec les pays de l'OCDE se sont développés au rythme de l'augmentation de sa part dans les échanges mondiaux. Les principaux partenaires commerciaux de la Chine sont l'Union européenne, les États-Unis et le Japon. La Chine est désormais le premier partenaire commercial du Japon et l'un des trois principaux partenaires d'une majorité d'économies de l'OCDE.

146. Le présent document se penche sur l'impact potentiel de la Chine sur les marchés mondiaux. Elle exerce notamment un impact planétaire au travers de sa participation aux réseaux internationaux de production. Les activités internationales de transformation ont été le moteur de la phénoménale croissance des échanges extérieurs de la Chine au cours des dernières décennies, moteur qui a été alimenté par la libéralisation des échanges et de l'investissement menée par le pays. Ce document examine la stratégie adoptée par la Chine, qui consiste à assurer l'expansion de ses exportations sur deux fronts. Le premier aspect de cette stratégie consiste à tirer parti du facteur de production qui constitue son atout majeur - sa main-d'œuvre surabondante - en privilégiant les produits manufacturés à forte intensité de main-d'œuvre, qui créent des emplois. Le second consiste à promouvoir son objectif de développement économique en modernisant son économie grâce à la production et à l'exportation de biens de plus haute technologie. Le pays paraît par ailleurs s'élever dans la chaîne de valeur.

147. La Chine a aussi un impact sur les prix mondiaux. Du fait de son rapide processus d'urbanisation, d'industrialisation et de construction d'infrastructures, le pays importe des quantités toujours croissantes de matières premières et de produits primaires, d'où des pressions à la hausse sur les prix mondiaux des principaux produits de base. Dans le même temps, l'abaissement des obstacles au commerce et la rapide augmentation de la productivité enregistrée en Chine dans le secteur manufacturier et celui de l'électronique ont abouti à une soudaine explosion des exportations, d'où une baisse de leur prix sur les marchés internationaux. Cette tendance perdurera sans doute tant que la Chine poursuivra son processus de "rattrapage". L'ampleur de cette dégradation des termes de l'échange de la Chine est cependant relativement modeste par rapport à la croissance globale de son PIB par habitant. Elle a eu pour contrepartie une augmentation des revenus et des niveaux de vie dans le reste du monde. En effet, l'importation à bas prix de produits manufacturés chinois a accru de près de $0.2 \%$ par an le revenu réel des consommateurs dans la zone OCDE. Par ailleurs, l'augmentation des prix des produits de base non pétroliers n'aura probablement qu'un impact limité sur les revenus dans les pays de l'OCDE, vu que des réponses du côté de l'offre se profilent déjà à l'horizon.

148. Afin de quantifier l'impact de la Chine sur l'économie mondiale, la dernière partie de ce document présente de nouvelles estimations établies à l'aide d'un modèle d'équilibre général, le modèle FTAP. Dans ce modèle, l'investissement direct étranger est traité sur une base bilatérale, ce qui est essentiel pour comprendre les conséquences de la libéralisation de l'économie chinoise, vu l'importance qu'y revêt la présence étrangère.

149. Le modèle constate que la Chine retire des gains notables de la mise en œuvre des engagements pris par elle à l'OMC ou d'une libéralisation des droits de douane et des obstacles aux échanges de services, 
alors qu'il n'en résulte qu'un impact assez limité sur les économies de l'OCDE. Compte tenu que la structure des échanges bilatéraux entre la Chine et les différentes économies de l'OCDE reflète des dissemblances dans la répartition des avantages comparatifs ainsi que des différences dans la structure des obstacles au commerce et dans la localisation géographique, la mise en œuvre par la Chine des engagements pris lors de l'accession à l'OMC ou d'une éventuelle libéralisation plus poussée devrait avoir, d'après les prévisions, un impact inégal sur les pays membres de l'OCDE. L'incidence la plus directe résultera probablement d'une amélioration des résultats à l'exportation des pays de l'OCDE qui commercent déjà avec la Chine ou y investissent massivement mais qui se heurtent toujours à d'importants obstacles du point de vue de l'accès au marché. Au vu de la configuration des échanges, l'impact lié à l'accès au marché sera probablement plus sensible pour la Corée, le Japon, l'Australie et la NouvelleZélande, mais demeurera sans doute limité en ce qui concerne les autres économies de l'OCDE.

150. La libéralisation de l'économie chinoise pourrait également avoir une incidence sur les économies de l'OCDE du fait du surcroît de compétitivité des exportateurs chinois, qui devraient bénéficier d'une baisse des coûts des produits intermédiaires et des services. Les parts de marché non négligeables de la Chine dans les importations des pays de l'OCDE laissent penser qu'une concurrence accrue avec les importations chinoises sera de fait une probable conséquence de la libéralisation en Chine. Cependant, ces effets liés à la compétitivité seront presque toujours compensés par les effets liés à l'accès aux marchés, ce qui procurera dans la majorité des cas, des gains globaux relativement modestes aux pays de l'OCDE. Il convient en outre de noter que l'analyse menée dans ce document ne tient pas compte des effets dynamiques de l'ouverture de la Chine et aboutit donc probablement à des estimations basses de l'impact de l'intégration de la Chine dans l'économie mondiale.

151. Les résultats de la modélisation dépendent toutefois de données sur la production, la consommation, les échanges et l'investissement qui datent de l'accession de la Chine à l'OMC et ils risquent donc de n'être qu'approximatifs du fait de la rapidité de l'évolution structurelle de l'économie chinoise et des relations entre la Chine et ses partenaires commerciaux. Il convient en outre de noter que notre analyse ne tient pas compte des effets dynamiques de l'ouverture de la Chine et aboutit donc probablement à des estimations basses de l'intégration de la Chine dans l'économie mondiale. 


\section{RÉFÉRENCES}

Adams, F. G., Ganges B. et Y. Shachmurove, "Why is China so Competitive? Measuring and Explaining China's Competitiveness", World Economy, vol. 29, n² 2, février 2006.

Adhikari, R. et Y. Yang, (2002), "What will WTO membership mean for China and its trading partners?" Finance \& Development, vol. 39, n 3, septembre 2002.

AIE (2005), Oil Market Report, 12 avril 2005.

ANASE (2004), "Statistics of Foreign Direct Investment in ASEAN", sixième édition, Investment \& Enterprise Unit Bureau for Economic Integration, Secrétariat de l'ANASE 2004.

Arayama, Y. et K. Miyoshi, "Regional Diversity and Sources of Economic Growth in China", The World Economy, vol. 27, n 10, pp. 1583-1607, novembre 2004.

Arndt, S. (2004), Trade Integration and Production Networks in Asia: The Role of China, Claremont McKenna College, juin 2004.

Bach, C., Martin, W. et J. Stevens (1996), China and the WTO: Tariff offers, exemptions and welfare implications, Weltwirtschaftliches Archiv 132 (3), 409-31.

Banque mondiale (2005), Indicateurs du développement dans le monde, Banque mondiale, août 2005.

Banque mondiale, (1994), China foreign trade reform, A World Bank country study, La Banque mondiale, Washington, DC.

Banque mondiale, (2004), China: An Evaluation of World Bank Assistance, 2004.

Base de données statistiques de l'ONU sur le commerce de marchandises (COMTRADE).

Bhattasali, D., Li S. et W. Martin, (dir. de pub.) (2004), China and the WTO: Accession, Policy Reform, and Poverty Reduction Strategies, Banque mondiale, Washington, DC, 2004.

Bouet, A., Fontagné L., Mimouni, M. et X. Pichot (2002), Market Access Maps for GTAP: A Bilateral Measure of Merchandise Trade Protection, GTAP Resource Paper $n^{\circ} 1045$.

Bureau d'information du Conseil d'État (2005), White Paper on IPR Protection, disponible sur www.china.org.cn au 27 février 2006.

Calabrese, J. (2005), "Dragon by the Tail: China's Energy Quandary", Washington, DC, Middle East Institute, http://www.mideasti.org/pdfs/calabrese304.pdf

Centre de recherche sur le développement (2004), Intellectual Property Right System: Challenge and Countermeasure, Centre de recherche sur le développement, Conseil d'État de la République populaire de Chine, 2004. 
CNUCED (2003), "China: an emerging FDI outward investor", e-brève, CNUCED, Genève, 4 décembre 2003.

CNUCED (2004), Rapport sur l'investissement dans le monde 2004 : La montée en puissance du secteur des services, Genève, 2004.

CNUCED (2005a) Rapport sur l'investissement dans le monde 2005: Les sociétés transnationales et l'internationalisation de la recherche-développement, Genève, 2005.

CNUCED (2005b), Rapport sur le commerce et le développement, 2005.

Commission européenne (2004), “The Challenge to the EU of a Rising Chinese Economy”, Rapport 2004 sur la competitivité européenne, SEC, 2004, 1397, 2004.

Dee, P. et K. Hanslow (2000), "Multilateral Liberalisation of Services Trade", Productivity Commission Staff Research Paper, Ausinfo, Canberra, 2000.

Dougherty, S. et R. Herd, (2005) "Fast-Falling Barriers and Growing Concentration: The Emergence of a Private Economy in China", Document de travail du Département des affaires économiques, $\mathrm{n}^{\circ} 471$, ECO/WKP(2005)58, OCDE, Paris, 16 décembre 2005.

Economy, E. (2005), "China's Rise in Southeast Asia: implications for the US”, Journal of Contemporary China, 14(44), août 2005.

EIU (2005) Country Report China, EIU, septembre 2005.

Feyzioglu, T. et L. Willard (2006), "Does Inflation in China Affect the US and Japan", Document de travail du FMI, WP/06/36, FMI, Washington, DC, février 2006.

FMI (2004), Perspectives de l'économie mondiale, FMI, avril 2004.

FMI (2006), Statistiques de la balance des paiements, base de données en ligne.

François, J. et D. Spinanger (2002), Regulated Efficiency, WTO Accession and the Motor Vehicle Sector in China, document rédigé pour la conférence sur le thème "WTO Accession, Policy Reform and Poverty Reduction", Beijing, 28-29 juin.

Garten, J. (2004), “China: The Missing Member at the G-8 Table”, YaleGlobal Online, 3 juin 2004.

Gaulier, G., Lemoine, F. et D. Unal-Kesenci (2005), China's Integration in East Asia: Production Sharing, FDI \& High-Tech Trade, Centre d'études prospectives et d'informations internationales (CEPII), 9 juin 2005.

Gilbert, J. et T. Wahl (2002), "Applied General Equilibrium Assessments of Trade Liberalisation in China", The World Economy, vol. 25, n 5 , mai 2002.

Gilboy, G. J. (2004) “The Myth behind China’s Miracle”, Foreign Affairs, juillet/août 2004.

Gill, B. et S. Tay (2004), Partners and Competitors: Coming to terms with the U.S. - China economic relationship, Centre for Strategic and International Studies, Washington: DC, avril 2004. 
Hanslow, K., Phamduc T. et G. Verikios (1999), “The Structure of the FTAP Model”, Staff Research Memorandum, Australian Productivity Commission.

http://www.chinabusinessreview.com/public/0601/overmyer.html

Hu, Z., et Khan, M. S. (1997), Why is China Growing So Fast?, Economic Issues n 8, Fonds monétaire international, Washington, D.C, 1997.

IACC (2006), Special 301 Recommendations, Submission of the International Anti-Counterfeiting Coalition, Inc., to the US Trade Representative, Washington, DC, 10 février 2006.

Ianchovichina, E., Suthiwart-Narueput, S. et M. Zhao, (2004) "Regional Impact of China's WTO Accession", in K. Krumm, et H. Kharas, East Asia Integrates: A Trade Policy Agenda for Shared Growth, Washington, DC : Banque mondiale, janvier 2004.

Jones, C. I. (2004), Growth and Ideas, Document de travail $n^{\circ} 10767$, National Bureau of Economic Research, Cambridge, MA, USA, septembre, 2004.

Kaplinski, R. (2005), Globalisation, Poverty and Inequality: Between a Rock and a Hard Place, Cambridge: Polity Press, 2005.

Lardy, N. (2002) Integrating China into the Global Economy, Washington, DC: Brookings Institution Press, 2002.

Lejour, A. (2000), China and the WTO: the impact on China and the world economy, 2000.

Li, S. et F. Zhai, (2002), China's WTO Accession and Implications for its Regional Economies, Économie Internationale, vol. 92 (2002), pp. 67-102.

Li, Y. (2002), “China's Accession to WTO: Exaggerated Fears?" Genève : CNUCED, Document de réflexion $\mathrm{n}^{\circ} 165$, novembre 2002.

Lin, J. Y. (2004), Lessons of China's Transition from a Planned Economy to a Market Economy, Document de travail $\mathrm{n}^{\circ} \mathrm{E} 2004001$, Centre chinois de recherche économique, 2 février 2004.

Mai, Y., Horridge M. et F. Perkins, (2003), Estimating the Effects of China's accession to the World Trade Organisation, document présenté à la sixième Conférence annuelle sur l'analyse économique mondiale, 12-14 juin 2003, Scheveningen, La Haye, Pays-Bas, 2003.

Malcolm G. (1998), “Adjusting Tax Rates in the GTAP Database." GTAP technical paper $\mathrm{n}^{\circ}$ 12, Center for Global Trade Analysis, Université Purdue, West Lafayette, 1998.

Mallon, G. et J. Whalley, (2004), China's Post Accession WTO Stance, Cambridge, MA : document de travail du NBER (National Bureau of Economic Research) n 10649, août 2004.

Mattoo, A. (2003), China's Accession to the WTO: The Services Dimension, Journal of International Economic Law 2003 6(2):299-339; doi:10.1093/jiel/6.2.299.

Ministère du Commerce des États-Unis, Major Shippers Report by Category, 6/2005 data, http://www.otexa.ita.doc.gov/msr/cat0.htm 
Molnar, M. (2005), China Embraces Globalisation: Strengthening Integration in Trade and Investment, présenté à la conférence de 2005 de la LACEA à l'Université américaine de Paris, 29 octobre 2005.

Morrison, W. (2005a) China-U.S. Trade Issues, CRS Issue Brief for Congress, 25 mai 2005.

Morrison, W. (2005b) China's Economic conditions, CRS Issue Brief for Congress, $1^{\text {er }}$ juillet 2005.

$\mathrm{Mu}$, Q. et K. Lee (2005), Knowledge diffusion, market segmentation and technological catch-up: The case of the telecommunication industry in China, Elsevier Policy Research, février 2005.

OCDE (2002a), La Chine dans l'économie mondiale, OCDE, Paris, 2002.

OCDE (2002b), L'impact économique mondial de l'accession de la Chine à l'OMC, CCNM/TD(2002)10/REV1, 2002.

OCDE (2003) Chine : progrès et enjeux de la réforme, OCDE, Paris, 2003.

OCDE (2004), Document de travail $\mathrm{n}^{\circ} 4$ de l'OCDE sur les politiques commerciales - "Ajustement structurel dans les industries du textile et du vêtement dans l'environnement commercial de l'aprèsATV”, [TD/TC/WP(2004)23/FINAL], 2004.

OCDE (2005a) Études économiques de l'OCDE : Chine, OCDE, Paris, 2005.

OCDE (2005b), Perspectives de l'investissement international, OCDE, Paris, 2005.

OCDE (2005c), "Les droits de propriété intellectuelle en Chine: enjeux et perspectives en matière de gouvernance", La gouvernance en Chine, OCDE, Paris, 2005.

OCDE (2005d), "Macroeconomic Policies: New Issues of Interdependence", Centre de développement de l'OCDE, document de travail n² 241, [DEV/DOC(2005)1], 20 janvier 2005.

OCDE (2005e), Science, technologie et industrie : Tableau de bord de l'OCDE, Paris, 2005.

OCDE (2005f), Review of Agricultural Policies in China, OCDE, Paris, 2005.

OCDE (2005g), Promoting IPR Policy and Enforcement in China: Summary of OECD-China Dialogues on Intellectual Property Rights Policy and Enforcement, Paris, 2005.

OCDE (2005h), Information Technology Outlook 2006: China - ICT Supply, Demand and ICT Policy [DSTI/ICCP/IE(2005)12/Chap4], 8-9 décembre 2005.

OCDE (2005i), Évolution des capacités mondiales d'acier, [DSTI/SU/SC(2005)15], OCDE, 17 octobre 2005.

OCDE (2005j), Modal Estimates of Services Barriers, TD/TC/WP(2005)36, 8 novembre 2005.

OCDE (2005k), Perspectives économiques de l’OCDE, volume 2005/2, n 78, décembre 2005.

OCDE (2006a), Chine : Politiques ouvertes envers les fusions et acquisitions, OCDE, Paris, 2006.

OCDE (2006b), Les dépenses publiques en Chine : Vers plus d'efficacité et d'équité, OCDE, Paris 2006. 
OMC (2001a), “Achèvement des négociations sur l'accession de la Chine à l'OMC”, Communiqué de presse de l'OMC, Genève, 17 septembre 2001.

OMC (2001b), Article 18 du "Protocole d'Accession de la République populaire de Chine", WTO, [WT/L/432], 23 novembre 2001.

OMC (2003), Comprendre l'OMC, septembre 2003.

OMC (2004), Statistiques du commerce mondial, 2004.

OMC (2006), Examen des politiques comerciales - Rapport du secrétariat - République populaire de Chine, $\mathrm{OMC}, 2006$.

Overmyer, M. (2006), "WTO: Year Five", China Business Review, janvier 2006, http://www.chinabusinessreview.com/public/0601/overmyer.html

Park, W. et D. Lippoldt (2003), "Incidence des droits de propriété intellectuelle sur l'investissement direct étranger et le commerce dans les pays en développement", Documents de l'OCDE, vol. $3, \mathrm{n}^{\circ} 11$, document 294, 2003.

Park, W. et D. Lippoldt (2005), "Licences internationales et renforcement des droits de propriété intellectuelle dans les pays en développement au cours des années 90", Revue économique de l'OCDE, vol. 40, Paris, 2005.

Phamduc, T. (2000), "The Construction of a Bilateral Capital Stocks Matrix for GTAP Industries", Document de travail du FTAP, 2000.

Polouektov, A. (2002), "The Non-Market Economy Issue in International Trade in the Context of WTO Accession", UNCTAD/DITC/TNCD/MISC.20, 9 octobre 2002.

Prasad, E. (dir. de pub.) (2004), China's Growth and Integration into the World Economy: Prospects and Challenges, FMI, Étude spéciale n² 232, 2004.

Puga, D. et D. Trefler (2005), Wake Up and Smell the Ginseng: The Rise of Incremental Innovation in LowWage Countries, document de travail du NBER n 11571, août 2005.

Qin, J. (2003), "WTO-Plus" Obligations and Their Implications for the World Trade Organization Legal System: an Appraisal of the China Accession Protocol", Journal of World Trade, vol. 37, 2003.

Rodrik, D. (2006), “What's so special about China's exports?" document de travail du NBER n 11947 , Cambridge, MA, janvier 2006.

Rumbaugh, T. et N. Blancher, "International Trade and the Challenges of WTO Accession", in E. Prasad (dir. de pub.) (2004) China's Growth and Integration into the World Economy: Prospects and Challenges, Étude spéciale n 232, FMI, Washington: DC, 2004.

Sachs, J. et W. T. Woo, (2003), "China's Economic Growth after WTO Membership", Journal of Chinese Economic and Business Studies, 2003, vol. 1, n 1, 2003.

Shafaeddin, S. M. (2004), "Is China's accession to WTO threatening exports of developing countries?" China Economic Review, vol. 15, 2004. 
Steinberg, W. (2006), Monitor with No Teeth: An Analysis of the WTO China Trade Review Mechanism, $1^{\text {er }}$ janvier 2006, http://blj.ucdavis.edu/article/578/.

US-China Economic and Security Review Commission (2004), "China in the World Trade Organization: Compliance, Monitoring, and Enforcement”, Rapport au Congrès de 2004, juin 2004.

US-China Economic and Security Review Commission (2005), "The Trade Lawyers Advisory Group Paper Re: Statutory Criteria for Changing China's Non-Market Economy Status”, document préparé pour l'US-China Economic and Security Review Commission, 18 août 2005. www.uscc.gov/researchpapers.

USCIB (2005), Submission to the US Trade Representative (USTR) on China's Compliance with its World Trade Organization (WTO) Commitment, USCIB, New York, NY, 2005.

USTR (2005), 2005 Report to Congress on China's WTO Compliance, US Trade Representative, 2005.

USTR (2005), National Trade Estimate Report on Foreign Trade Barriers, mars 2005, http://www.ustr.gov/assets/Document_Library/Reports_Publications/2005.

USTR (2006), U.S.-China Trade Relations: Entering a New Phase of Greater Accountability and Enforcement, Top-to-Bottom Review, USTR, Washington, DC, février 2006.

Verikios, G. et X. Zhang (2000), "Sectoral Impact of Liberalising Trade in Services", document présenté à la troisième conférence sur l'analyse économique mondiale, Melbourne, 27-30 juin 2000. Disponible à l'adresse suivante : www.monash.edu.au/policy/conf/53Verikios.pdf

Walmsley, T., Hertel, T et E. Ianchovichina (2004), Assessing the Impact of China's WTO Accession on Investment, Document de travail du GTAP, 2004.

Wang Y. et G. Wang (2005), "China", in P. Macrory, Appleton, A., et Plummer, M., World Trade Organization: Legal, Economic and Political Analysis, OMC, Genève : Springer, (2005), vol. 111, 2005.

Whalley, J. (2003), Liberalization in China's Key Service Sectors Following WTO Accession: Some Scenarios and Issues of Measurement, Document de travail $n^{\circ} 10143$, Cambridge, MA: National Bureau of Economic Research, 2003.

Yang, Y. (2003), China's Integration in the World Economy: Implications for Developing Countries, Document de travail WP/03/245, Washington, DC, FMI, 2003.

Yoshitomi, M. (2003), Post-Crisis Development Paradigms in Asia, Banque asiatique de développement, Manille, chapitre 3, 2003.

Yu, Y. (2005), “G-20 and China: A Chinese Perspective”, China \& World Economy, vol. 13, n 1, 2005.

Zhang, Y. (2005), China Goes Global, Foreign Policy Centre, Londres, avril 2005.

Zweig, D. et B. Jianhai (2005), “China's Global Hunt for Energy”, Foreign Affairs, septembre/octobre 2005. 


\section{ANNEXE 1: ANALYSE DES OBSTACLES SECTORIELS DANS LES SERVICES}

1. Cette annexe décrit en détail les engagements de libéralisation pris par la Chine tels qu'ils figurent dans sa liste de l'AGCS. Nous avons calculé des indices de restriction des échanges pour évaluer le degré de mise en œuvre des engagements de la Chine dans cinq secteurs de services (banque, assurance, télécommunications, distribution et services d'ingénierie). ${ }^{1}$ Les principales composantes de ces indices de restriction des échanges (IRE) sectoriels sont présentées ci-dessous, tout comme les modifications apportées aux politiques appliquées imputables aux engagements pris à l'OMC. Une note a été attribuée aux différentes composantes de l'IRE sur la base des engagements pris par la Chine dans le cadre de l'AGCS compte tenu (i) de l'état de la situation en 2001 (date de l'accession), et (ii) de l'état de la situation en 2008 (date à laquelle tous les engagements de libéralisation auront été mis en œuvre). L'analyse sectorielle ci-dessous dépeint les modifications apportées aux politiques mises en œuvre en Chine, alors que les IRE (diagrammes A1.1 à A1.6) offrent une représentation graphique de ces variations. Les graphiques montrent que la mise en œuvre des engagements pris à l'OMC dans le secteur bancaire ramènerait le degré de restriction en vigueur au sein de celui-ci à un niveau inférieur à la moyenne de l'OCDE; par contre, dans tous les autres secteurs, malgré d'importantes mesures de libéralisation, les indices de restriction des échanges demeurent supérieurs à la moyenne de l'OCDE (mais inférieurs à leur niveau dans la plupart des pays en développement pris en considération dans cette étude). Les équivalents de taxes dans les secteurs de services correspondants associés à la mise en œuvre des engagements pris à l'OMC dans ces cinq secteurs de services apparaissent au tableau A5.4. Les réductions des équivalents de taxes indiquées au tableau A5.4 sont utilisées dans l'exercice de simulation pour modéliser la libéralisation des échanges de services selon les modes 1 et 3.

\section{Banque et assurance}

\section{Composantes de l'IRE}

- Modes 1 et 2 : restrictions aux prêts et emprunts transnationaux, restrictions aux opérations transnationales d'assurance, conditions d'établissement ou de résidence/reconnaissance pour fournir des services financiers transfrontaliers, obligation de coopération avec des sociétés locales, restrictions géographiques, restrictions liées au champ d'activité, obligation d'obtenir un agrément, restrictions à l'achat de services financiers à l'étranger,

- Mode 3: limites imposées à la participation étrangère, restrictions concernant le mode d'établissement, dont les prescriptions en matière de coentreprise, procédures de sélection et d'autorisation, restrictions au champ d'activité des entreprises; limitations en matière d'ouverture de nouveaux établissements dans les secteurs de la banque et de l'assurance, et

- Mode 4 : limitation de la durée de séjour et du nombre de permis de travail, problèmes liés aux conditions d'agrément et de reconnaissance, restrictions imposées au conseil d'administration.

\section{Modifications apportées aux politiques mises en auvre dans le secteur bancaire}

- Secteur bancaire : l'état de la situation en 2001

Cette approche est décrite en détail in OCDE (2005j) "Modal Estimates of Services Barriers", TD/TC/WP(2005)36, 8 novembre 2005. 
2. La Chine compte parmi les pays dont l'indice de restriction des échanges est supérieur à la moyenne. En l'occurrence, le degré de restriction découle en partie des modes 1 et 3 . Dans le cas du mode 1, les emprunts transnationaux sont subordonnés à la demande et à l'obtention d'une autorisation, et le montant des ressources en devises que les banques étrangères peuvent se procurer à l'étranger est limité au moyen d'un système de quotas. Pour ce qui est du mode 3, il convient de souligner les limites imposées à la participation étrangère car elles impliquent un degré de restriction des échanges extrêmement élevé. Une institution financière étrangère donnée ne peut détenir plus de $20 \%$ du capital d'une institution financière chinoise. En outre, le pourcentage total de la participation au capital détenue par plusieurs institutions financières étrangères est en pratique limité à $25 \%$. Il faut en outre signaler l'existence de restrictions concernant l'extension géographique des activités bancaires (et notamment de celles de prêt). Les restrictions géographiques et en matière de clientèle liées aux opérations en devises ont été abolies en 2001, mais les banques étrangères ne pouvaient opérer que dans certaines régions et n'accepter de dépôts que de non résidents, et ce exclusivement en devises, l'octroi de prêts aux citoyens chinois leur étant en outre interdit. Pour ce qui est de l'agrément, bien que les listes de concessions prévoient certes qu'il doit être exclusivement accordé selon des critères prudentiels, mais les règles qui régissaient les institutions financières à capitaux étrangers stipulaient que chaque banque étrangère ne pouvait ouvrir plus d'une succursale par an, et que toute institution financière à capitaux étrangers dont la demande aurait été rejetée devait attendre au moins une année avant de pouvoir renouveler sa demande (cette condition a été abrogée en 2004 à la suite de la révision des Règles de mise en cuvre du règlement de la République populaire de Chine régissant les institutions financières à capitaux étrangers (voir ci-dessous) publiée par la Commission chinoise de régulation du secteur bancaire. Enfin, les procédures de sélection et d'autorisation constituent également un important obstacle.

- Secteur bancaire : mise en œuvre des engagements pris à l'OMC (2008)

3. Conformément aux engagements pris par la Chine dans le cadre de l'AGCS, le secteur bancaire sera pleinement libéralisé dès 2006. Les restrictions géographiques aux activités seront progressivement supprimées avant 2006. Les opérations en monnaie locale seront autorisées dès 2003 avec les entreprises chinoises et en 2006 avec tous les clients. Pour ce qui est de l'agrément, le 26 juillet 2004, la Commission chinoise de régulation du secteur bancaire a publié des Règles révisées de mise en œuvre du Règlement de la République populaire de Chine régissant les institutions financières à capitaux étrangers (désignées ciaprès sous le nom de "nouvelles Règles"), lesquelles sont entrées en vigueur le $1^{\text {er }}$ septembre 2004. Dans une tentative pour abaisser les seuils d'entrée sur le marché pour les banques étrangères, les nouvelles Règles ont abrogé les conditions imposées dans les Règles antérieures, telles que celles qui prévoyaient que chaque banque étrangère ne pouvait ouvrir plus d'une succursale au cours d'une même année et que toute institution financière à capitaux étrangers dont la demande aurait été rejetée devait attendre au moins une année avant de pouvoir renouveler sa demande. Les nouvelles Règles ont également sensiblement assoupli les normes que les succursales chinoises de banques étrangères doivent respecter en matière de fonds propres pour pouvoir fournir des services en monnaie locale (Renminbi) aux entreprises et aux particuliers de nationalité chinoise, elles ont réduit le nombre des normes de fonds propres imposées aux succursales chinoises de banques entièrement détenues par des intérêts étrangers et de banques en coentreprise sino-étrangères, et elles ont également abaissé le volume minimal de fonds propres dont elles doivent disposer. 
Diagramme A1.1. IRE dans le secteur bancaire

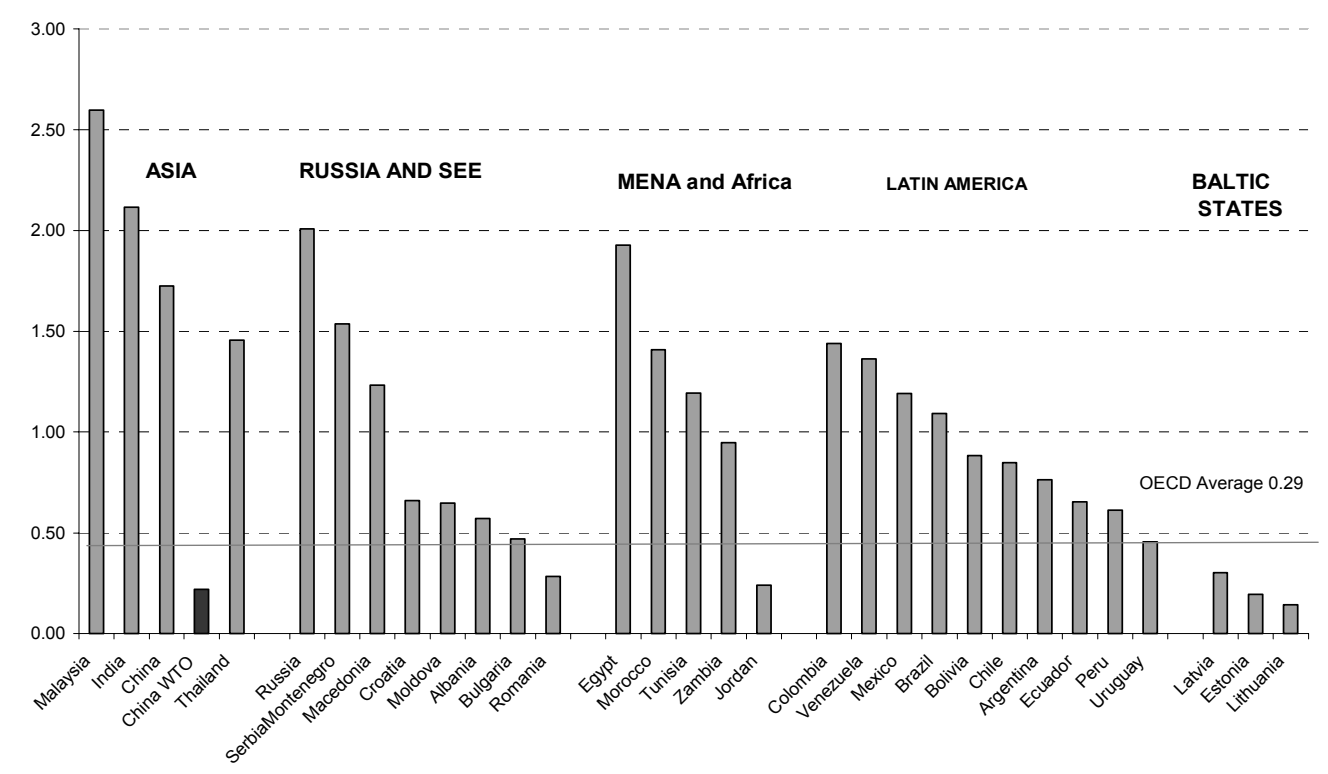

Source : Calculs des auteurs sur la base de la méthode décrite in OCDE (2005j) et des réformes des politiques sectorielles indiquées à l'annexe 1.

\section{Modifications apportées aux politiques mises en auvre dans le secteur de l'assurance}

- $\quad$ Assurance : l'état de la situation en 2001

4. L'indice élevé de restriction des échanges de la Chine dans les services d'assurance peut principalement s'expliquer par les plafonds qui y sont imposés à l'IDE (limitation à 50\% des participations étrangères au capital des coentreprises d'assurance-vie et à $51 \%$ pour ce qui est de celles d'assurance non vie), par les restrictions à la forme de la présence commerciale (seules les succursales et les coentreprises sont autorisées), par les restrictions géographiques (au moment de son accession à l'OMC, la Chine n'a autorisé les sociétés étrangères d'assurance vie et non vie à s'établir que dans seulement cinq villes), par les restrictions au champ d'activité (les sociétés étrangères d'assurance non-vie ne sont autorisées à mener que certaines formes d'activités dans le secteur non-vie et ne peuvent fournir d'assurance-vie qu'aux seuls particuliers et non à des groupes), ainsi que par les conditions d'agrément (en vertu des engagements pris par la Chine dans le cadre de l'AGCS, l'agrément ne sera accordé que sur la base de règles prudentielles, sans restrictions numériques ni critères discrétionnaires concernant les besoins économiques, mais certaines conditions additionnelles devront toutefois être respectées pour obtenir un agrément). Pour pouvoir déposer une demande d'agrément, les sociétés d'assurance étrangères doivent en effet respecter trois conditions ${ }^{2}$ : les sociétés d'assurance à capitaux étrangers (i) doivent être établies depuis plus de 30 ans dans un pays membre de l'OMC; (ii) doivent avoir un bureau de représentation en Chine depuis au moins 2 ans et (iii) devront disposer d'un volume total d'actifs de plus de 500 millions RMB à la fin de l'année précédant la demande. Les entreprises nationales ne sont par contre pas tenues de remplir ces conditions pour déposer une demande d'agrément. Certaines restrictions s'appliquent en outre aux modes 1 et 2 : restrictions à la fourniture transfrontières, sauf pour ce qui est de l'assurance et la réassurance internationales dans le domaine de la navigation maritime, de l'aviation et des transports (MAT) ainsi que des services de courtage liés à l'assurance internationale dans ce même domaine, comme en ce qui

2 Aux termes de l'article 8 des Règles régissant l'administration des sociétés d'assurance à capitaux étrangers (Rules on the Regulation for the Administration of foreign invested insurance companies) en vigueur depuis le $1^{\mathrm{er}}$ février 2002 sur www.circ.gov.cn. 
concerne l'assurance et la réassurance des risques commerciaux de grande ampleur, restrictions à la consommation de services de courtage à l'étranger, prescriptions en matière de cessions de réassurance. Par ailleurs, aucune "association d'assureurs" ne figure sur la liste des personnes morales autorisées à émettre des contrats d'assurance et de réassurance.

- Assurance : mise en œuvre des engagements pris à l'OMC (2008)

5. Conformément aux engagements pris par la Chine dans le cadre de l'AGCS, le secteur de l'assurance sera libéralisé dès 2004 , à l'exception du seuil maximal de $50 \%$ concernant la participation étrangère dans le domaine de l'assurance-vie. Les mesures de libéralisation s'échelonneront selon le calendrier suivant : les restrictions à l'établissement dans le segment de l'assurance non-vie devraient être levées en 2003, les restrictions géographiques et celles concernant le champ d'activité en 2004, les prescriptions en matière de cession étant abolies en 2005. Néanmoins, contrairement à ce qui se produit dans le secteur bancaire, où les limites imposées à la participation étrangère sont totalement supprimées, une limitation à 50\% de la participation étrangère sera maintenue dans le segment de l'assurance-vie. Dans le secteur de la banque comme dans celui de l'assurance, l'agrément sera exclusivement accordé selon des critères prudentiels.

Diagramme A1.2. IRE dans le secteur de l'assurance

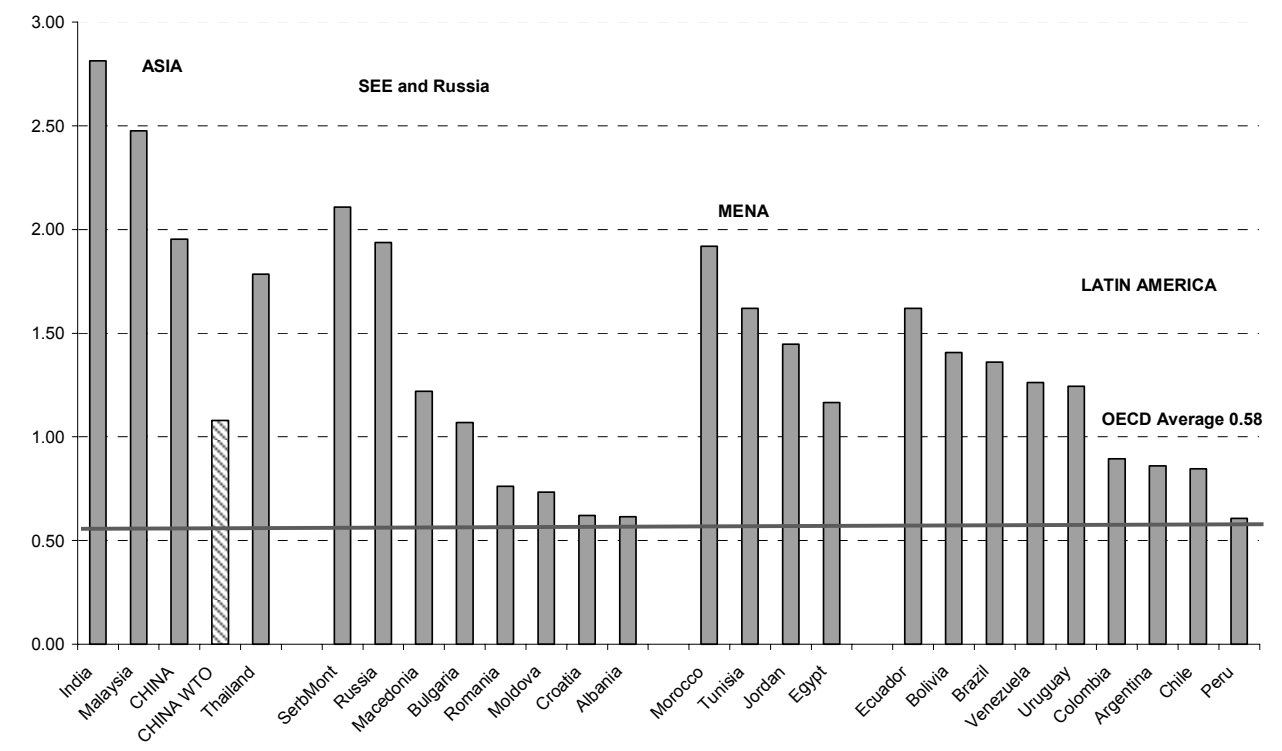

Source : Calculs des auteurs sur la base de la méthode décrite in OCDE (2005j) et des réformes des politiques sectorielles indiquées à l'annexe 1.

\section{Télécommunications (fixes et mobiles)}

\section{Composantes de l'IRE}

- Mode 1 : restrictions à la fourniture de lignes louées ou de réseaux, restrictions au raccordement de lignes louées et de réseaux privés au réseau téléphonique public commuté (RTPC), restrictions aux services de revente simple internationale et de téléphonie IP.

- Mode 2 : restrictions aux services de rappel (call back). 
- Mode 3: limites imposées à la participation étrangère, prescriptions concernant le degré de concurrence, et notamment en matière de coentreprise, procédures de sélection et d'autorisation, restrictions aux activités commerciales, restrictions à l'octroi de l'agrément.

- Mode 4 : limitation de la durée de séjour et du nombre de permis de travail, problèmes liés aux conditions d'agrément et de reconnaissance, restrictions imposées au conseil d'administration.

\section{Modifications apportées aux politiques mises en auvre dans le secteur des télécommunications}

- Télécommunications : l'état de la situation en 2001

6. Le secteur chinois des télécommunications est caractérisé par d'importants obstacles à l'entrée (limitation de la participation étrangère à $30 \%$ dans les télécommunications à valeur ajoutée, $25 \%$ dans les services mobiles de téléphonie et de transmission de données), obligation de créer une coentreprise pour entrer sur le marché, restrictions géographiques et restrictions au champ d'activité des sociétés de télécommunications. Qui plus est, d'importantes restrictions sont liées à la fourniture de lignes louées ou de réseaux et au raccordement au RTPC de lignes louées et de réseaux privés.

- Télécommunications : mise en œuvre des engagements pris à l’OMC (2008)

7. D'importantes évolutions sont intervenues dans ce secteur depuis 2001. La plupart des restrictions géographiques ont été supprimées en 2002 et 2003. Par ailleurs, les limites à l'investissement étranger ont été portées à $49 \%$ dans les télécommunications à valeur ajoutée et les services mobiles de téléphonie et de transmission de données. En vertu des engagements pris par la Chine dans le cadre de l'AGCS, toutes les restrictions géographiques seront éliminées dès 2007 et les limites imposées à l'investissement étranger dans les télécommunications fixes seront assouplies. La participation étrangère majoritaire ne sera pas autorisée. Aucun engagement à autoriser la fourniture transfrontières de services de télécommunications n'a été pris.

Diagramme A1.3. IRE dans le secteur des télécommunications fixes

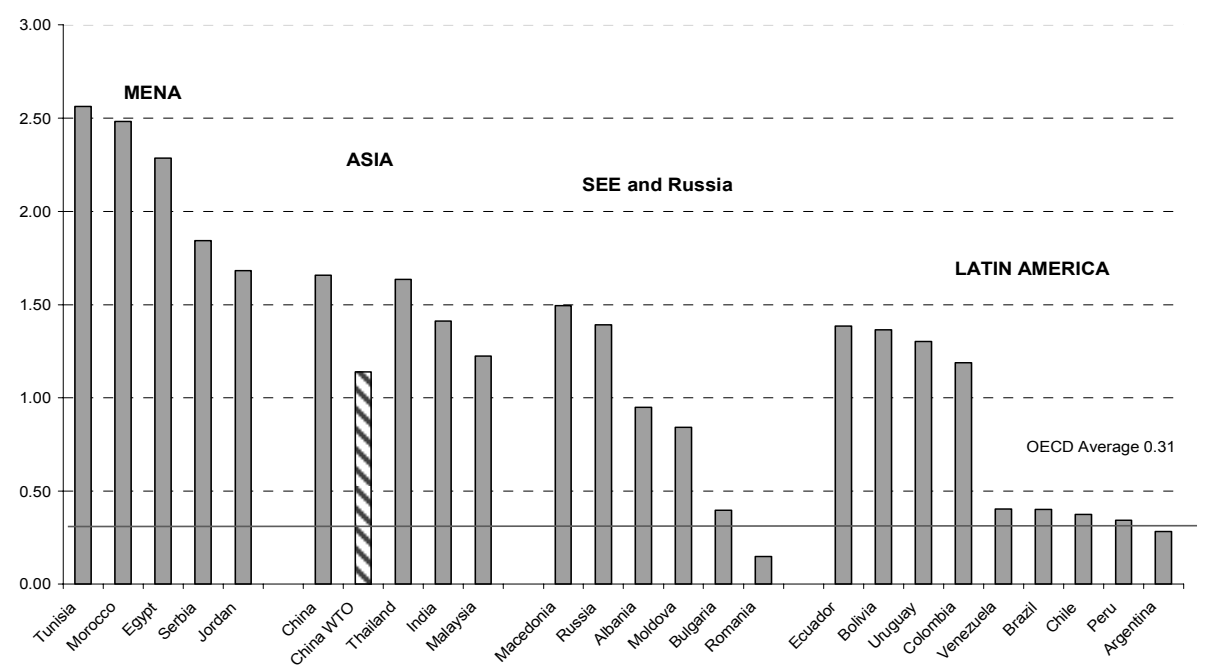

Source : Calculs des auteurs sur la base de la méthode décrite in OCDE (2005j) et des réformes des politiques sectorielles indiquées à l'annexe 1. 
Diagramme A1.4. IRE dans le secteur des télécommunications mobiles

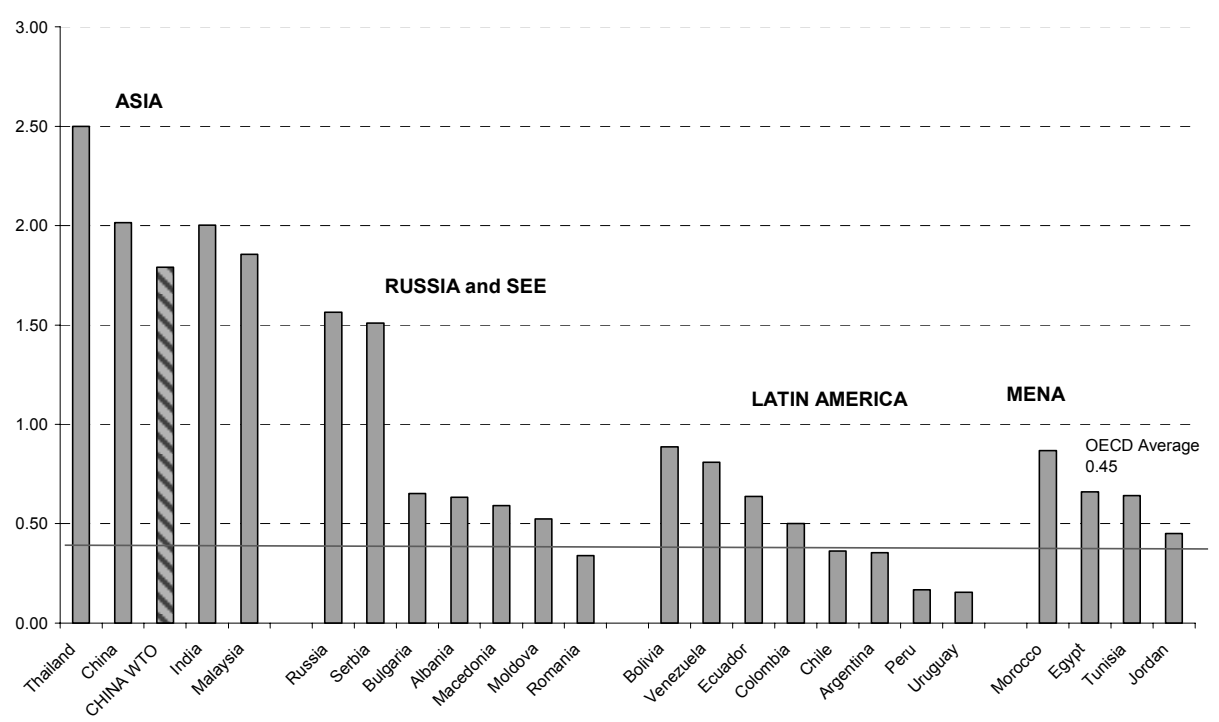

Source : Calculs des auteurs sur la base de la méthode décrite in OCDE (2005j) et des réformes des politiques sectorielles indiquées à l'annexe 1.

\section{Services professionnels (ingénierie)}

\section{Composantes de l'IRE}

- Mode 1: restrictions à la fourniture de services transfrontières sur le marché (obligation d'établissement).

- Mode 2 : restrictions à l'achat par les consommateurs de services aux entreprises à l'étranger.

- Mode 3: limites imposées à la participation étrangère, partenariat/coentreprise/association avec un partenaire étranger, investissement et participation au capital des professionnels étrangers, pratiques multidisciplinaires, prescriptions concernant le degré de concurrence, et notamment en matière de coentreprise, procédures de sélection et d'autorisation, restrictions aux activités commerciales, restrictions à l'octroi de l'agrément.

- Mode 4: limitation de la durée de séjour et du nombre de permis de travail, restrictions imposées au conseil d'administration, agrément et accréditation des professionnels étrangers, résidence et présence sur place.

\section{Modifications apportées aux politiques mises en auvre dans le secteur de l'ingénierie}

- Ingénierie : l'état de la situation en 2001

8. L'ampleur de l'indice de restriction des échanges dans le secteur de l'ingénierie s'explique par les restrictions liées aux formes d'établissement et les prescriptions en matière de coentreprise (pour ce qui est du mode 3), par les conditions d'entrée, d'agrément et d'accréditation des professionnels et des dirigeants étrangers, ainsi que par les quotas et les critères économiques concernant le nombre de professionnels étrangers ou d'entreprises étrangères (dans le cas du mode 4). En ce qui concerne le mode 1, il n'existe certes aucun obstacle à l'élaboration de plans-masse, mais la coopération avec les organisations professionnelles chinoises est requise pour tous les autres segments.

- Ingénierie : mise en œuvre des engagements pris à l'OMC (2009) 
9. Conformément aux engagements pris par la Chine dans le cadre de l'AGCS, le secteur sera entièrement libéralisé dès 2006 (sauf pour ce qui est des restrictions mentionnées ci-dessus concernant le mode 1). Les restrictions aux formes d'établissement doivent être levées en 2006.

Diagramme A1.5. Services professionnels (ingénierie)

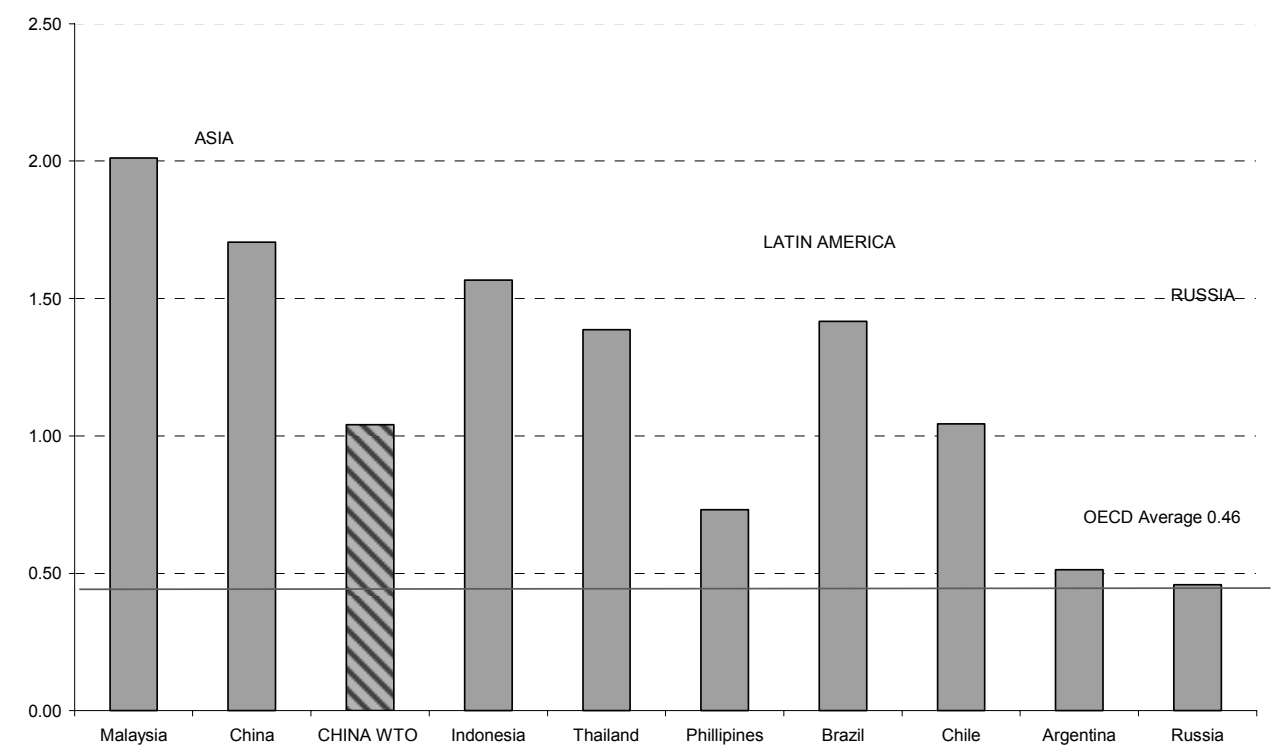

Source : Calculs des auteurs sur la base de la méthode décrite in OCDE (2005j) et des réformes des politiques sectorielles indiquées à l'annexe 1.

\section{Distribution}

\section{Composantes de l'IRE}

- Mode 1: restrictions à la fourniture de services transfrontières sur le marché (obligation d'établissement).

- Mode 2 : restrictions à l'achat par les consommateurs de services de distribution à l'étranger.

- Mode 3 : limites imposées à la participation étrangère, restrictions concernant les terrains à usage commercial, restrictions imposées aux grandes surfaces, système d'agrément pour les activités d'importation en gros, promotion des produits de détail, monopoles d'État - exclusions de produits, protection des droits de propriété intellectuelle.

- Mode 4: limitation de la durée de séjour et du nombre de permis de travail, obligation pour les dirigeants d'obtenir un agrément.

\section{Modifications apportées aux politiques mises en oeuvre dans le secteur de la distribution}

- Distribution : l'état de la situation en 2001

10. L'indice de restriction des échanges relativement élevé s'explique par les restrictions liées à aux formes d'établissement (l'entrée n'est autorisée que par le biais de coentreprises), les restrictions géographiques et les exclusions de produits (les livres, les journaux, les produits pharmaceutiques, les pesticides, les engrais chimiques ainsi que le pétrole brut et raffiné sont exclus).

- Distribution : mise en œuvre des engagements pris à l'OMC (2008) 
11. Conformément aux engagements pris par la Chine dans le cadre de l'AGCS, le secteur sera largement ouvert dès 2006. La participation étrangère majoritaire sera autorisée et aucune restriction géographique ou quantitative ne s'appliquera. Les seules restrictions qui subsistent ont trait aux échanges transfrontières et à la fourniture de deux produits. La Chine s'est en outre engagée à autoriser les entreprises à capitaux étrangers à distribuer leurs produits fabriqués en Chine et à fournir des services subordonnés. Par ailleurs, les fournisseurs de services étrangers seront autorisés à fournir tout l'éventail des services connexes et subordonnés, dont des services après-vente pour les produits dont ils assurent la distribution. Ces vastes engagements déterminent le niveau du nouvel IRE, qui est très proche de la moyenne de l'OCDE.

\section{Diagramme A1.6. IRE dans le secteur de la distribution}

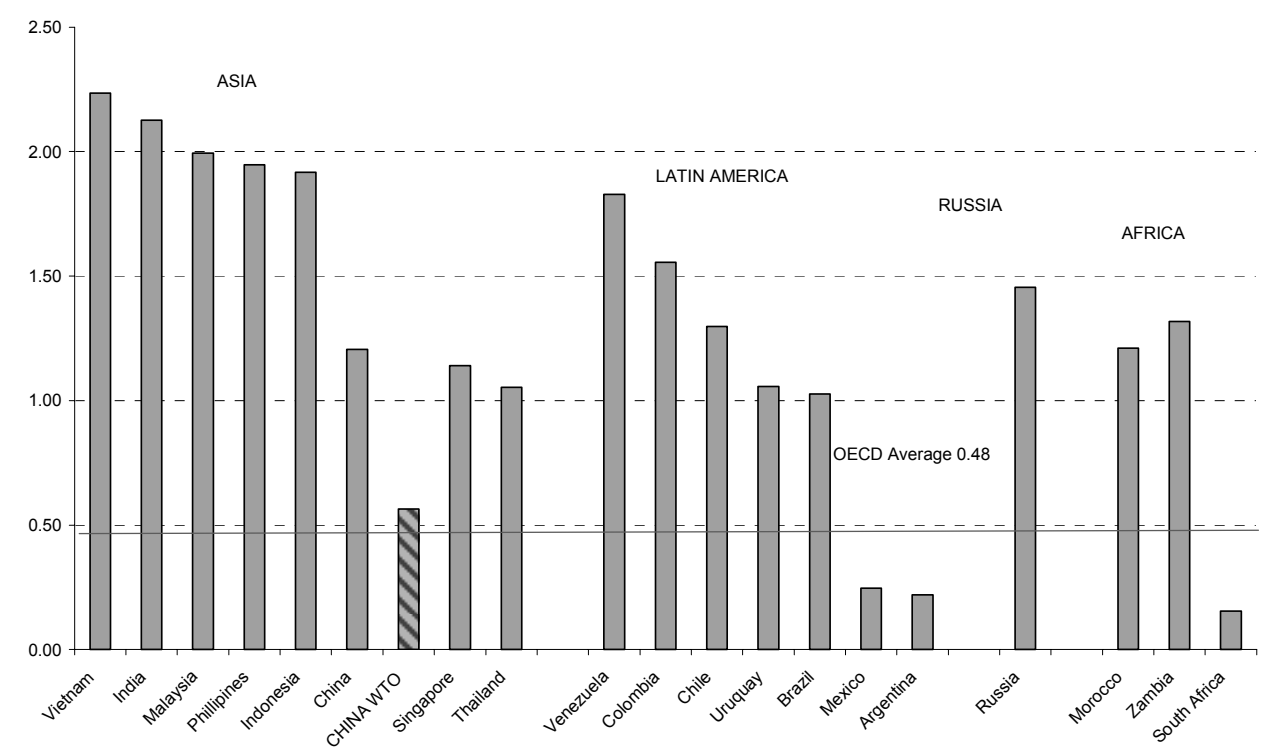

Source : Calculs des auteurs sur la base de la méthode décrite in OCDE (2005j) et des réformes des politiques sectorielles indiquées à l'annexe 1 . 


\section{ANNEXE 2: MODÉLISATION DE L'IMPACT DE L'INTÉGRATION DE LA CHINE DANS L'ÉCONOMIE MONDIALE, TOUR D'HORIZON DES TRAVAUX DÉJÀ PUBLIÉS SUR LE SUJET}

1. Depuis les années 90, l'intégration de la Chine dans l'économie mondiale a suscité un regain d'intérêt, d'où une augmentation du nombre d'études visant à quantifier l'impact des réformes commerciales liées à l'OMC menées par le pays. Le nombre de ces études ne reflète pas uniquement l'intérêt pour le pays, mais aussi le développement d'outils toujours plus élaborés pour mesurer cet impact. Cette augmentation a été rendue possible par les progrès de la théorie et des techniques analytiques ainsi que par le spectaculaire accroissement de la puissance de calcul et de traitement des données des ordinateurs.

${ }^{1}$ Cette annexe examine plusieurs études qui se sont attachées à estimer les effets qu'exerce sur l'économie chinoise son accession à l'OMC ainsi que l'incidence de celle-ci sur les autres pays en développement et les pays développés.

\section{Méthodologie}

2. Pour mesurer l'incidence macroéconomique de la libéralisation des échanges, il faut un cadre d'équilibre général mondial qui rende tout à la fois compte des effets intersectoriels au sein de chaque économie et des interactions entre les divers pays. L'approche d'équilibre général s'appuie sur des informations détaillées sur les dotations en facteurs, les structures économiques et les instruments d'action des pouvoirs publics. Toutes ces variables sont intégrées dans un cadre d'équilibre des marchés couvrant un ou plusieurs pays et une multiplicité de secteurs et offrant une représentation très élaborée des relations entre l'offre et la demande. Du fait qu'il simule les effets induits par l'évolution des politiques, des structures ou des marchés, un modèle EGC bien défini ${ }^{2}$ constitue un utile instrument d'analyse de l'impact économique, puisqu'il permet d'évaluer l'incidence des politiques sectorielles sur l'ensemble de l'économie.

3. On trouvera ci-dessous une vue d'ensemble et un examen des travaux actuels sur la mesure et la modélisation des effets de la libéralisation et de l'accession de la Chine à l'OMC. Il s'agit de présenter les principaux résultats auxquels parviennent les études réalisées en s'appuyant sur un modèle d'équilibre général calculable (EGC) et d'analyser ces résultats sur la base du cadre de modélisation sous-jacent, des données utilisées pour évaluer le degré de protection et de la manière dont elles sont incorporées dans les modèles et les scénarios supposés d'accession et de libéralisation. Ces études utilisent bien souvent le modèle GTAP mis au point à l'Université Purdue. ${ }^{3}$ Le modèle GTAP est un modèle statique d'allocation

1 Pour plus d'informations, voir R. Piermartini et R. Teh, (2005), "Demystifying Modelling Methods for Trade Policy", OMC, Genève, document de travail n $10,2005$.

2 Un modèle EGC se définit comme un système intégré d'équations (modèle d'équilibre général), dérivé de la théorie économique du comportement de tous les agents économiques, dont la solution simultanée s'appuie sur une base de données numérique pour déterminer les valeurs des variables endogènes. D. Schreiner, D. Marcouiller, G. Tembo, et E. Vargas, Computable General Equilibrium Modeling for Regional Analysis, Glossary of Terms, West Virginia University, disponible à l'adresse suivante : http://www.rri.wvu.edu/WebBook/Schreiner/glossary.htm.

3 Le modèle GTAP est un modèle standard d'équilibre général appliqué à l'échelle mondiale qui postule des marchés parfaitement concurrentiels et une technologie aux rendements d'échelle constants. Ce modèle représente les exigences des consommateurs au moyen d'une différence constante dans la forme des fonctions d'élasticité et, pour ce qui est de l'offre, il met l'accent sur le rôle que joue la mobilité intersectorielle des 
des ressources où les dotations en ressources et la technologie sont considérées comme données. Malgré son utilité pour l'analyse des possibles effets économiques de l'accession de la Chine à l'OMC, certaines des caractéristiques du modèle GTAP, telles que l'hypothèse de rendements d'échelle constants ou celle de la mobilité intersectorielle de la main-d'œuvre, ainsi que le fait qu'il s'agit d'un modèle statique imposent certaines réserves concernant les résultats des exercices de modélisation. Certains travaux empiriques ont cependant adapté le modèle GTAP en vue de tenir compte des rendements d'échelles croissants et des obstacles à la mobilité de la main-d'œuvre. Diverses études se sont par ailleurs attachées à analyser à l'aide d'un modèle dynamique les effets économiques de l'accession de la Chine à l'OMC. Quelques études s'appuient ainsi sur le modèle G-CUBED de l'Asie-Pacifique élaboré par l'Université nationale australienne. Le modèle G-CUBED ${ }^{4}$ est un modèle dynamique qui examine les liens explicites entre les marchés de capitaux, et donc l'investissement, et les secteurs réels.

\section{Études quantitatives pertinentes}

4. La présente section se penche brièvement sur deux examens détaillés des ouvrages sur le sujet OCDE (2002a) et Gilbert et Wahl (2002) - puis passe en revue plusieurs études qui revêtent un intérêt du point de vue des travaux décrits à la section III du présent document. Ces deux examens détaillés et une des études en question, OCDE (2002b), ont été effectués avant que la Chine n'adhère à l'OMC et avant que les concessions consenties par le pays dans le cadre de l'accord d'accession à l'OMC ne soient connues. La Direction des échanges de l'OCDE a réalisé une synthèse et une comparaison de 16 grandes études rédigées entre 1996 et 2000 estimant les conséquences pour l'économie chinoise de son accession à l'OMC. ${ }^{5}$ Cet examen par l'OCDE des études mesurant les conséquences de l'adhésion de la Chine à l'OMC a permis de constater qu'elles parvenaient à des conclusions très différentes, mais que la majorité d'entre-elles s'accordaient à considérer qu'il en résulterait d'importants gains de bien-être globaux pour l'économie chinoise comme pour le monde dans son ensemble. Il paraissait toutefois probable que la libéralisation des échanges ne fasse pas que des gagnants. La plupart des études s'attendaient à ce que les secteurs chinois du textile et de l'habillement bénéficient grandement de l'accession à l'OMC, du fait de la suppression progressive, après l'accession du pays à l'OMC, du contingentement des exportations de la Chine dans le secteur du textile et de l'habillement. En revanche, certains secteurs de l'industrie lourde, tels que l'industrie automobile, et certains secteurs de l'agriculture exigeant beaucoup de terres (blé et maïs, par exemple) pourraient subir une contraction de leur activité du fait de l'adhésion de la Chine à l'OMC. La plupart des études circonscrivaient leur analyse aux conséquences des réductions tarifaires proposées par la Chine et de la suppression des contingents sur les exportations chinoises de textiles et de vêtements. Elles ne couvraient généralement pas toutes les mesures de libéralisation des échanges (telles que la levée des obstacles non tarifaires et la réduction des restrictions aux échanges de services) auxquelles la Chine s'est engagée dans le cadre des négociations commerciales bilatérales et multilatérales d'adhésion à l'OMC.

facteurs en tant que facteur déterminant la production des différents secteurs. La différenciation des produits selon qu'il s'agit de biens importés ou de biens produits au sein du pays et celle des importations en fonction de leur région d'origine rendent possible des échanges dans les deux sens dans chaque catégorie de produit, selon la facilité de substitution entre les produits provenant de différentes régions. La terre, le capital, le travail qualifié et non qualifié et, dans certains secteurs, les ressources naturelles constituent les facteurs de production employés et ils sont supposés être pleinement utilisés. Voir https:/www.gtap.agecon.purdue.edu/.

$4 \quad$ Pour plus d'informations, voir W. J. McKibbin et P. Wilcoxen (1995), "The theoretical and empirical structure of the G-Cubed model", Economic Modelling, 16 (1), pp. 106-148, http://www.msgpl.com.au/msgpl/download/struct.pdf

5 Voir OCDE (2002a), op. cit., "Synthèse d'études sur les conséquences de l'adhésion de la Chine à l'OMC", pp. 871-900. 
5. Gilbert et Wahl (2002) examinent 30 études effectuées entre 1994 et $2000 .^{6}$ Ils constatent qu'une réforme des échanges permettra à la Chine d'accroître son bien-être net, alors qu'elle n'aura sans doute que relativement peu de conséquences sur le bien-être net des autres économies. Les gains estimés dont devrait bénéficier la Chine à la suite d'une réforme unilatérale et de l'accession à l'OMC sont de l'ordre de 6 à 30 milliards USD (soit de 0.5 à $2.4 \%$ du PIB). Le gain de bien-être net retiré par le reste du monde d'une réforme unilatérale de l'économie chinoise ou de son accession à l'OMC se situerait d'après les estimations dans une fourchette de 8 à 35 milliards USD. Sur le plan sectoriel, l'une des grandes conclusions qui se dégagent est que l'expansion des exportations chinoises de textiles et de vêtements à la suite de l'expiration de l'AMF devrait entraîner un déclin de ces industries dans les pays développés.

6. L'OCDE (2002b) ${ }^{7}$ a évalué l'impact de l'accession de la Chine à l'OMC sur les pays voisins d'Asie. Cette étude s'appuyait sur un modèle macroéconomique dynamique plurinational et plurisectoriel - le modèle G-Cubed de l'Asie-Pacifique - qui rend compte des liens qui unissent la Chine et ses partenaires commerciaux par le biais des échanges de biens et de services et des flux de capitaux. Ce modèle permet une couverture détaillée des pays de la région et met en évidence les liens puissants qui existent entre les pays par le biais des marchés de produits et d'actifs. Chose importante, il facilite en outre une analyse des effets entraînés par les variations des flux d'IDE à destination de la Chine. L'étude présentait trois scénarios. Le premier correspond au cas "naï", qui ne tient compte que de la réduction des obstacles aux échanges de la Chine. Le second envisage le "détournement des flux d'IDE" et se distingue de la simulation naïve par une réduction de 1 point de pourcentage de la prime de risque exigée par les investisseurs étrangers en Chine. Le troisième scénario, celui d'un "IDE ayant des retombées technologiques", prend en considération les gains de productivité totale des facteurs (PTF) dont bénéficieraient en Chine les secteurs produisant des biens manufacturés durables ou non durables comme celui des services, ainsi que la baisse de la PTF qu'enregistreraient les secteurs producteurs de biens en Indonésie, en Malaisie, aux Philippines et en Thaïlande. Il convient de noter que l'étude ne modélise pas de manière directe l'impact de la libéralisation dans le domaine des services et de l'investissement mais analyse néanmoins comment les gains de productivité dans le secteur des services - qui constituent une conséquence implicite de leur libéralisation - accroissent le taux de rendement du capital, et se répercutent ainsi sur le reste de l'économie.

7. Les résultats d'ensemble du cas naïf indiquent qu'aussi longtemps que la suppression des obstacles commerciaux en Chine ne s'accompagnera pas d'un détournement des flux d'IDE au profit du pays, son adhésion à l'OMC n'aura d'importants effets positifs sur le plan économique que pour l'économie chinoise. Le scénario de détournement des flux d'IDE donne des résultats d'ensemble qualitativement comparables à ceux du cas naïf dans le long terme, à la grande différence près que les effets quantitatifs sur la Chine sont amplifiés. Dans ce scénario de détournement des flux d'IDE, à long terme, le PIB dépassera de $5 \%$ son niveau de référence, ce qui représente le double de l'effet à long terme de $2.5 \%$ observé dans le cas naïf. Les écarts de PIB et de consommation par rapport aux niveaux de référence sont tous inférieurs à $1 \%$ pour les autres pays analyses. Dans le troisième scénario, celui d'un IDE ayant des retombées technologiques, les sorties d'IDE ralentissent le progrès technologique alors que les entrées d'IDE l'accélèrent. Les résultats globaux font apparaitre des gains considérables pour la Chine (d'après les estimations, le PIB est à long terme supérieur de $25 \%$ à son niveau de référence) et des pertes notables pour les quatre pays de l'ANASE-4 (Indonésie, Malaisie, Philippines et Thaïlande : les pertes de PIB dans le long terme s'élèvent à $7 \%$ pour la Thaïlande, à $5 \%$ pour la Malaisie et les Philippines et à $3 \%$ pour l'Indonésie). Le modèle montre que l'impact sur les autres économies est très faible, sauf pour celle de Hong Kong - Chine. La forte croissance de la Chine ne paraît pas avoir d'effets positifs sur la croissance

\footnotetext{
6 Voir J. Gilbert et T. Wahl, “Applied General Equilibrium Assessments of Trade Liberalisation in China”, The World Economy, vol. 25, cinquième édition, mai 2002, pp. 697-731.

7 Voir OCDE (2002b), L'impact économique mondial de l'accession de la Chine à l'OMC, CCNM/TD(2002)10.
} 
de ses autres partenaires commerciaux non membres de l'ANASE. Le PIB du Japon n'aura ainsi progressé que de $0.4 \%$ en 2020 , celui de la Corée de $0.6 \%$ et celui du Reste de la zone OCDE de $0.3 \%$.

8. L'étude parvient à la conclusion que le principal effet pour la Chine de son adhésion à l'OMC est de grandement renforcer sa sécurité économique en lui assurant un meilleur accès aux marchés extérieurs et en accroissant sa fiabilité en tant que fournisseur sur ces derniers. Un meilleur accès aux marchés extérieurs a pour effet de réduire la prime de risque exigée par les investisseurs dans les secteurs chinois qui travaillent pour l'exportation, stimulant ainsi les entrées d'IDE (en détournant une partie des flux d'IDE de certains autres pays d'Asie). L'augmentation du volume d'IDE, synonyme de considérables retombées technologiques, permet à l'économie chinoise de bénéficier de très importants gains de productivité et de bien-être. L'étude ne fait apparaître aucune évolution majeure de la structure des exportations de la plupart des partenaires commerciaux de la Chine, à quelques notables exceptions près : certains de ses voisins d'Asie orientale qui se trouvent en concurrence avec la Chine sur les marchés d'exportation de biens à forte intensité de main-d'œuvre et qui rivalisent avec elle pour attirer des investissements directs étrangers dans les usines qui produisent ces biens pourraient avoir à supporter des effets négatifs.

9. Le FMI (2004) a examiné l'émergence de la Chine et son impact sur l'économie mondiale. ${ }^{8}$ Cette étude utilise la version 5 du GTAP, l'année 1997 étant prise pour référence, mais la base de données étant néanmoins mise à jour à l'aide de données datant de 2002 sur les échanges bilatéraux et les agrégats macroéconomiques. ${ }^{9}$ Les auteurs s'attachent à quantifier l'impact qu'exercent sur les termes de l'échange et les flux commerciaux des différentes régions divers scénarios concernant le profil d'évolution de la croissance en Chine jusqu'en 2020. Ils ont construit deux scénarios. Dans le premier scénario - celui d'une "rapide intégration de la Chine" - les variables économiques sont supposées enregistrer une progression à peu près conforme aux projections à long terme (du FMI, de la Banque mondiale et de l'ONU). En Chine, la productivité, l'emploi et le capital physique et humain sont censés continuer à bénéficier d'une croissance accélérée, grâce à un rythme de réforme soutenu et à une absorption rapide de la main-d'œuvre rurale dans le secteur moderne de l'économie. Dans un tel scénario, l'économie chinoise serait capable d'absorber environ 150 millions de travailleurs additionnels, issus pour une large part des zones rurales agricoles. En conséquence, la production réelle de la Chine s'accroîtrait de près de $8 \%$ par an, et sa part de la production et des échanges mondiaux serait plus que doublée d'ici 2020. Pour ce qui est des économies développées, leur part dans la production mondiale diminuerait, du fait tout à la fois du processus de rattrapage dont bénéficient les pays en développement sur le plan de la productivité et du vieillissement de la population dans les économies plus prospères.

10. Un second scénario - celui d'une "lente intégration de la Chine" - a été élaboré en vue d'isoler l'impact qu'exerce sur l'économie mondiale la rapide intégration de la Chine. Ce scénario part de l'hypothèse que la part de la Chine dans la production mondiale se maintient aux niveaux actuels et que sa part dans les échanges mondiaux n'évolue guère. Ce scénario n'est pas considéré comme une possibilité réaliste d'évolution de la Chine. Sa comparaison avec le premier scénario permet par contre de déterminer

8 FMI (2004), "L'émergence de la Chine et ses retombées sur l'économie mondiale", Ch. 2, Perspectives économiques mondiales, FMI, avril 2004.

9

Les auteurs ont construit une version couvrant dix régions et sept secteurs. Les dix régions sont les suivantes : i) la Chine; ii) les économies développées; iii) les NEI : Hong Kong-Chine, Corée, Singapour et Taipei chinois ; iv) l'ANASE; v) l'Asie du sud; vi) l'Afrique sub-saharienne; vii) le Mexique, la Colombie et le Venezuela ; viii) tous les autres pays en développement de l'hémisphère occidental ; ix) le Moyen-Orient et l'Afrique du nord; et x) le reste du monde. Les sept secteurs sont les suivants : i) agriculture ; ii) industries extractives ; iii) textiles et habillement; iv) industries manufacturières à forte intensité de main-d'œuvre non qualifiée; v) industries manufacturières à forte intensité de main-d'œuvre qualifiée ; vi) services à forte intensité de main-d'œuvre non qualifiée ; et vii) services à forte intensité de main-d'œuvre qualifiée. 
quels sont ceux qui gagneront et ceux qui perdront du fait de la poursuite de la rapide croissance chinoise. Chose importante, tous les autres pays sont supposés se caractériser par leur souplesse structurelle et pouvoir s'adapter sans heurts aux différents taux de croissance enregistrés par la Chine.

11. L'étude parvient à la conclusion que la Chine qui tirera elle-même les plus grands avantages de sa propre croissance mais que l'impact sur le reste du monde considéré globalement n'en sera pas moins également positif. Les économies industrielles bénéficieront d'une baisse des coûts de leurs importations à forte intensité de main-d'œuvre et d'une augmentation de la demande de biens et services à forte intensité de qualifications. Les pays développés bénéficieront quant à eux de possibilités accrues d'exportation vers la Chine, tant de produits primaires que de produits manufacturés destinés à subir une nouvelle transformation avant d'être réexportés. Les pays dont les dotations en facteurs sont similaires à celles de la Chine et qui sont les plus proches concurrents de celle-ci sur les marchés mondiaux en ressentiront les effets. Pour éviter des pertes considérables, ces pays devront procéder à d'importants ajustements et faire preuve de souplesse sur les marchés de produits et de main-d'œuvre.

12. Ianchovichina et Martin (2004) estiment l'impact de l'accession à l'OMC qui résulte de la libéralisation dans l'agriculture, le secteur manufacturier et les services et analysent les perspectives qu'ouvre la suppression des contingents auxquels étaient soumises les exportations chinoises de textiles et de vêtements. ${ }^{10}$ Les auteurs utilisent la base de données GTAP, version 5, élaborée avec des données de 1997 et de meilleures estimations du degré de protection dans l'agriculture et les services. Chose importante, les auteurs utilisent des mesures de la libéralisation des échanges de marchandises établies en fonction de l'accord multilatéral final, alors que pour la libéralisation des services, ils réduisent de moitié les équivalents de taxes estimés par François et Spinanger (2000) au moyen d'un modèle gravitationnel. Pour les besoins de ces simulations, le modèle GTAP standard a été ajusté de manière à tenir compte des activités de transformation pour l'exportation menées en Chine (les ristournes de droits de douane sont prises en considération) ainsi que les principaux mécanismes de son marché du travail (les obstacles à la mobilité de la main-d'œuvre entre les activités rurales et urbaines sont expressément pris en considération dans le modèle).

13. Ianchovichina et Martin établissent une distinction entre les effets de l'accession de la Chine à l'OMC selon qu'ils correspondent à la libéralisation engagée entre 1995 et 2001 dans la perspective de l'accession ou à celle entreprise à partir de 2001 pour se conformer aux engagements pris lors de l'accession. Pour ce qui est de la libéralisation intervenue après 2001, ils constatent une importante augmentation du volume des échanges par rapport à celui de la production, les exportations totales s'accroissant de $17 \%$. Sans surprise, la croissance la plus rapide est enregistrée par les exportations du secteur de l'habillement, qui ont progressé de plus de $100 \%$ à la suite de l'abolition des contingents d'exportation. Les auteurs font cependant remarquer que l'expansion des exportations de textiles et de vêtements constitue un avantage différé du Cycle d'Uruguay dont la Chine n'avait pu bénéficier avant $2001 \mathrm{du}$ fait qu'elle n'était pas membre de l'OMC. Ils constatent une augmentation des exportations agricoles du fait d'une baisse du coût des intrants agricoles mais aussi en raison des obstacles qui empêchent la main-d'œuvre agricole de quitter ce secteur d'activité. Les exportations progressent également dans le secteur automobile grâce aux gains d'efficience enregistrés dans ce secteur, qui tirent parti d'économies d'échelle et d'un plus grand rayonnement commercial. Les importations s'accroissent dans les secteurs qui bénéficient de sensibles réductions des obstacles aux échanges, dont les boissons et le tabac, les produits alimentaires transformés, les textiles, l'habillement, les oléagineux, les produits laitiers et le sucre.

10 E. Ianchovichina et W. Martin, "Economic Impacts of China's Accession to the WTO”, in Bhattasali, D., S. Li et W. Martin, (dir. de pub.), (2004) op. cit, pp. 211-236. 
14. Quant aux mesures de libéralisation prises au cours de la première période, de 1995 à 2001, les auteurs constatent qu'elles ont abouti à d'importants gains de bien-être globaux. Ils estiment que la réduction du degré de protectionnisme entreprise dans cette période permet de bénéficier durablement d'un gain d'environ 30 milliards USD par an (soit une augmentation d'environ $2.2 \%$ du revenu réel par habitant). La libéralisation entreprise entre 2001 et l'année où aura été menée à terme la mise en œuvre des engagements pris lors de l'accession générera des gains supplémentaires de 10 milliards USD par an au profit du pays. Ianchovichina et Martin font observer que leur évaluation des gains en termes de croissance des exportations et des revenus aboutit à des estimations basses. Ces estimations ne tiennent en effet pas compte des avantages qui découlent de l'abolition des obstacles non tarifaires et elles comportent d'importantes distorsions imputables à l'agrégation. ${ }^{11}$

15. L'étude suggère que les économies qui procèdent à des réformes et leurs partenaires commerciaux les plus proches sont les principaux bénéficiaires de l'accession de la Chine à l'OMC. La Chine en tirera les plus grands avantages dans la mesure où elle entreprend les réformes de plus grande envergure. Les auteurs ont observé que l'impact sur nombre de pays de l'OCDE partenaires commerciaux de la Chine était positif. Les gains les plus importants en termes absolus échoient à l'Amérique du nord et à l'Europe occidentale, près de la moitié des gains étant générés par la suppression des contingents imposés par ces pays aux exportations chinoises dans les secteurs des textiles et de l'habillement. Bien que l'étude ait constaté que l'Amérique du nord, l'Europe occidentale et le Japon bénéficient également des réductions du degré de protection de la Chine, les variations de l'utilité par habitant qui leur sont associées sont extrêmement faibles, puisqu'elles se situent dans une fourchette de 0.0 à $0.2 \%$. Ianchovichina et Martin notent que leur étude ne prend en considération ni l'impact de l'accession sur l'IDE ni les gains d'efficience dans les services qui résultent de l'augmentation des investissements dans ce secteur, ces gains étant difficiles à mesurer.

16. Walmsley et al (2004) étudient le lien entre l'accession à l'OMC et l'investissement en Chine au cours des deux prochaines décennies. Les auteurs utilisent une extension récursive dynamique du modèle GTAP standard - à savoir le modèle GTAP-Dyn. - qui est encore élargie pour prendre en compte les exonérations de droits sur les intrants et les biens d'équipement importés utilisés dans la production des exportations chinoises. Ce modèle complète la théorie de l'investissement en tenant compte de l'accumulation de capital, de la mobilité internationale du capital ainsi que de l'origine internationale des capitaux. Les simulations se fondent sur une agrégation en 11 régions et 13 secteurs de la base de données GTAP, version 4, et les effets sont examinés sur la période allant de 1995 à 2020. Les scénarios impliquent une réduction progressive de la prime de risque escomptée par les investisseurs étrangers, la suppression des droits de douane et des contingents conformément à ce qui est indiqué dans l'accord d'accession, la libéralisation du commerce international de services (modélisée comme un gain de productivité dont l'ampleur est mesurée au moyen des équivalents de taxes estimés par François et Spinanger), des gains de productivité dans le secteur automobile (modélisés comme une augmentation de $20 \%$ de la productivité totale des facteurs), ainsi que des impacts sur la productivité de chacun des secteurs à la suite de la libéralisation des règles régissant la présence commerciale étrangère (modélisés sous la forme de chocs asymétriques qui aboutissent en 2010 à une productivité totale des facteurs dans le secteur des services d'un niveau supérieur de $4.58 \%$ à celui de l'année de référence). L'accession est supposée commencer à prendre effet en 2002. Le délai nécessaire à la mise en œuvre de l'accord est en outre supposé être de 5 ans, avec des effets sur la participation étrangère et sur les revenus versés qui devraient durer jusqu'en 2020.

17. L'effet global sur le PIB qui en résulte (augmentation de $22.5 \%$ d'ici 2020 du fait de l'accession à l'OMC) est bien plus important que prévu dans les études antérieures. La majeure partie des gains de bienêtre $(84 \%)$ découlent des gains de productivité qui sont supposés intervenir dans le secteur automobile et celui des services. Par contre, les scénarios de libéralisation des droits de douane et des contingents

11 D. Bhattasali, et al., op. cit., p. 12. 
aboutissent tous à des résultats comparables à ceux des autres études. Les auteurs soulignent toutefois que, compte tenu de l'incertitude relative à l'impact de l'accession sur la productivité des secteurs de services, ce devrait être là un important domaine de recherches futures.

18. Ce tour d'horizon des travaux a examiné l'impact que l'intégration de la Chine dans l'économie mondiale exerce sur cette dernière, afin de fournir une vue d'ensemble des estimations disponibles et des modèles utilisés pour les obtenir. L'examen montre que l'accession de la Chine à l'OMC ou la libéralisation unilatérale à laquelle elle a procédé génèrent des gains de bien-être globaux quelles que soient les hypothèses de modélisation retenues. Les résultats sont difficiles à comparer eu égard aux différences tenant à la structure des modèles, aux chocs liés aux politiques mises en œuvre et aux données relatives au degré de protection utilisées dans ces simulations. Certaines conclusions n'en peuvent pas moins être tirées.

19. Une importante conclusion s'en dégage, à savoir que la Chine est le principal gagnant quelles que soient les hypothèses de modélisation retenues - elle enregistre en effet d'importants gains de bien-être allant de 0.4 à $22.5 \%$ de son PIB. Les études dynamiques qui prennent en considération les effets additionnels liés à l'investissement font apparaître des gains plus importants, tout comme celles qui tiennent compte des améliorations de la productivité associées à la libéralisation de la Chine. Par contre, l'impact sur le reste du monde en général et sur les pays de l'OCDE en particulier demeure limité dans toutes les études analysées (il est presque toujours inférieur à 1\% de variation du PIB). 
TD/TC/WP(2006)10/FINAL

\section{ANNEXE 3: STRUCTURE DES ÉCHANGES ET DES FLUX D'INVESTISSEMENT DIRECT ÉTRANGER ENTRE LA CHINE ET LES PAYS DE L'OCDE}

\section{Flux d'échanges entre la Chine et les pays de l'OCDE}

1. La présente section vise à décrire la structure des échanges de marchandises entre la Chine et les grandes économies de l'OCDE et les obstacles tarifaires auxquels ils se heurtaient en 2001. Elle est également utile pour interpréter les résultats des simulations de la mise en œuvre par la Chine de ses engagements à l'OMC ainsi que du scénario d'une totale libéralisation des obstacles tarifaires en vigueur dans le pays.

2. En 2001, la Chine comptait pour $6 \%$ des exportations mondiales et pour $4 \%$ des importations mondiales. Ces pourcentages occultent toutefois de considérables variations dans l'importance que revêt la Chine pour les différentes économies de l'OCDE et pour les divers marchés internationaux de biens et de services. Pour ce qui est de l'importance de la Chine en tant que marché de destination des exportations de la zone OCDE (tableau A2.1), les facteurs régionaux paraissent jouer un rôle important, les pays de l'OCDE les plus proches de la Chine tendant à exporter vers ce marché des parts plus élevées de leurs exportations totales (7\% pour l'Australie, $6 \%$ pour la Nouvelle-Zélande, $11 \%$ pour le Japon, 16\% pour la Corée, par exemple) que ce n'est le cas des États-Unis (3\%), du Canada (4\%) ou de l'UE (2\%).

3. La part du PIB du pays d'origine que représentent les exportations vers la Chine est particulièrement élevée en Corée (6.7\%), en Nouvelle-Zélande (2.2\%), en Australie (1.5\%) et au Japon (1.2\%), et elle tend là encore à être plus faible au Canada $(0.7 \%)$, aux États-Unis $(0.3 \%)$, dans l'UE-15 (0.6\%) et au Mexique $(0.2 \%)$. Dans l'ensemble, ces chiffres donnent à penser que la dépendance à l'égard de la Chine en tant que marché de destination des exportations était assez limitée en 2001, hormis pour les économies de la zone OCDE situées en Asie et en Océanie. À titre de comparaison, les exportations des États-Unis vers l'UE-15 comptaient en 2001 pour $29 \%$ de l'ensemble de leurs exportations et pour $2.7 \%$ de leur PIB, alors que les exportations de l'UE-15 vers les États-Unis représentaient $11 \%$ de l'ensemble de ses exportations ${ }^{1}$ et aux alentours de $3.7 \%$ de son PIB.

4. Les écarts entre les différents secteurs sont encore plus marqués que ceux entre les divers pays. La Chine compte ainsi pour une part importante des exportations de produits de l'agriculture et de la pêche d'un certain nombre de pays de l'OCDE (Australie 15\%, Nouvelle-Zélande 12\%, Canada et États-Unis 7\% chacun, Corée $6 \%$, Japon $5 \%$ ) et pour une part encore plus grande des exportations de ressources naturelles du Japon (43\%), de l'Australie (16\%), de la Corée (15\%), de la Nouvelle-Zélande (11\%) et du Mexique (7\%). Nombre de secteurs industriels enregistrent des parts élevées, dont en particulier les textiles, l'habillement et le cuir, qui sont perçus comme le principal secteur d'exportation chinois. La Chine absorbe en effet respectivement 56, 27, 8 et 7\% des exportations du Japon, de la Corée, de l'Australie et de la Nouvelle-Zélande dans le secteur des textiles, de l'habillement et du cuir. Une situation comparable peut être observée en ce qui concerne les substances et produits chimiques, dont la Chine absorbe respectivement $35,15,7$ et $5 \%$ des exportations en provenance de la Corée, du Japon, de l'Australie et de la Nouvelle-Zélande. Dans l'UE-15, aux États-Unis, au Canada, ou encore au Mexique, ces parts sont généralement inférieures à $10 \%$, sans être pour autant négligeables. La Chine compte ainsi pour $5 \%$ des exportations des États-Unis dans les secteurs des produits métalliques et du matériel électronique.

Y compris les échanges internes à l'UE-15. 
5. La part de la Chine dans les importations des différentes économies est en règle générale plus élevée que sa part dans leurs exportations ( $8 \%$ pour l'Australie, 5\% pour la Nouvelle-Zélande, 14\% pour le Japon, $9 \%$ pour la Corée, $9 \%$ pour les États-Unis, $4 \%$ pour le Canada, et 3\% pour l'UE-15). Cela donne à penser qu'une libéralisation plus poussée de la Chine pourrait avoir une incidence sur les secteurs des économies de la zone OCDE qui sont confrontés à la concurrence des importations (ainsi que sur les pays tiers qui sont en concurrence avec la Chine sur les marchés de l'OCDE) au travers des gains de compétitivité des exportateurs chinois. La part du PIB de l'économie correspondante que représentent les importations en provenance de Chine est particulièrement élevée pour la Corée (3.3\%), l'Australie et la Nouvelle-Zélande $(1.6 \%)$, le Japon (1.4\%) et le Canada (1.2\%) alors qu'elle tend à être moindre pour les États-Unis (1.1\%), l'UE-15 (0.9\%) et le Mexique (0.5\%). Malgré des différences selon les pays membres de l'OCDE, les secteurs où les importations en provenance de Chine occupent une place particulièrement importante incluent les textiles et l'habillement (où la part des importations varie de 5 à $65 \%$ ), les autres produits manufacturés (de 6 à 24\%), les machines et équipements (de 2 à 18\%), le matériel électronique (de 4 à $16 \%$ ) et dans une moindre mesure les produits métalliques (de 1 à $12 \%$ ) et les véhicules et pièces automobiles (de 0 à $14 \%$ ).

6. À l'extérieur de la zone OCDE, Hong Kong, Chine, dépend dans une large mesure du marché chinois dans des secteurs tels que les suivants : substances et produits chimiques $(61 \%$ des exportations de Hong Kong, Chine), machines et équipements (51\%), matériel électronique (33\%), produits métalliques $(39 \%)$, autres produits manufacturés $(26 \%)$, textiles et habillement $(24 \%)$ et produits alimentaires et boissons (11\%). Les importations en provenance de la Chine présentent de fait une structure similaire, ce qui donne à penser que ces flux sont dans une large mesure bidirectionnels et résultent très certainement de l'échange de produits et de leurs composants à différents stades de leur transformation. Pour la Russie, la Chine constitue une importante destination de ses exportations dans les secteurs suivants: machines et équipements $(28 \%)$, substances et produits chimiques $(14 \%)$, autres produits manufacturés $(10 \%)$ et produits métalliques (9\%). Les importations en provenance de la Chine comptent pour $31 \%$ des importations totales de la Russie dans les textiles, l'habillement et le cuir, pour 7\% dans les autres produits manufacturés et pour $6 \%$ dans l'agriculture et la pêche.

7. Les tableaux A3.3 et A3.4 présentent des informations détaillées sur la part des flux bidirectionnels dans les échanges de la Chine avec ses partenaires commerciaux. Les indices sectoriels globaux calculés pour l'ensemble des partenaires commerciaux qui figurent au tableau A2.4 suggèrent que les échanges de produits agricoles (indice des échanges intra-branche égal à 0.3 ) et de ressources naturelles $(0.2)$ dont le charbon (0.0) et le pétrole (0.1) sont pour l'essentiel unidirectionnels, et qu'il en va de même en ce qui concerne les textiles, l'habillement et le cuir (0.3) mais aussi les véhicules et pièces automobiles (0.4) ainsi que les autres produits manufacturés (0.4). Les échanges bidirectionnels semblent être relativement importants pour ce qui est des substances et produits chimiques (0.7), des produits métalliques $(0.7)$, des machines et équipements (0.7) et du matériel électronique (0.6).

8. Les indices des échanges intra-branche établis pour chacun des flux commerciaux bilatéraux et les coefficients de variation de ces indices calculés au niveau des produits et des pays font apparaître d'intéressantes différences dans la nature des relations commerciales bilatérales de la Chine. Compte tenu du niveau relativement élevé d'agrégation des produits auquel sont calculés ces indices dans notre étude, l'importance des pourcentages d'échanges intra-branche reflète aussi bien l'échange de différentes variétés d'un même produit que des "échanges verticaux" impliquant des échanges transfrontières d'un même produit à différents stades de transformation ou un échange de composants et de produits finis. Les données mettent néanmoins en évidence que les échanges de la Chine avec les économies de l'OCDE les plus proches (c'est-à-dire en l'occurrence le Japon, la Corée, l'Australie et la Nouvelle-Zélande) tendent à être plus bidirectionnels que ne le sont ses échanges avec les économies d'Amérique du nord ou d'Europe membres de l'OCDE. De considérables différences peuvent cependant être observées, même au sein d'une même industrie ou d'une même région. Par exemple, dans les véhicules et pièces automobiles, les échanges 
de la Chine avec la Corée se caractérisent par un important pourcentage d'échanges intra-branche alors que ce pourcentage est plutôt modeste dans le cas de ses échanges avec le Japon. Dans les textiles, l'habillement et le cuir, les échanges avec le Japon et la Corée tendent à être bidirectionnels alors que ceux avec les États-Unis et l'UE-15 sont essentiellement unidirectionnels. Ce dernier exemple met en évidence que les échanges régionaux pourraient se composer d'une plus grande part d'échanges d'un même produit à divers stades de la chaîne de production. Pour certains autres flux commerciaux bilatéraux tels que les échanges de la Chine avec l'UE-15 dans le secteur des produits alimentaires et des boissons, les pourcentages élevés d'échanges intra-branche sont sans doute plutôt dus à l'échange de différentes variétés de denrées alimentaires et de boissons produites par les partenaires commerciaux.

9. Les données sur les échanges bilatéraux de services actuellement incluses dans la base de données GTAP ne reflètent pas la structure des échanges de services entre les différents pays; les flux d'échanges de services sont ventilés selon diverses hypothèses et extrapolations des échanges de marchandises par pays partenaire. ${ }^{2}$ Toutefois, compte tenu que cette base de données constitue le soubassement sur lequel repose l'analyse des échanges de services transfrontières, une brève description de la structure des échanges de services est fournie ci-dessous.

10. Le commerce de gros constitue le secteur le plus important dans les échanges de la Chine en provenance et à destination des pays de l'OCDE (la part des importations de la Chine dans les exportations des pays de l'OCDE est supérieure à 7\% pour le Japon, la Corée, l'UE-15 et le Canada, alors que la part des exportations chinoises dans les importations des pays de l'OCDE est supérieure à 7\% pour le Japon, le Mexique et l'UE-15). Tous les autres secteurs enregistrent des valeurs plus faibles (entre $1 \%$ et $3 \%$ en règle générale) tant du point de vue de la part des importations de la Chine dans les exportations des pays de l'OCDE que de la part des exportations chinoises dans les importations des pays de l'OCDE.

11. De manière générale, les statistiques commerciales présentées aux tableaux A3.1 à A3.5 donnent à penser que les scénarios qui prennent en considération les améliorations de l'accès au marché chinois envisagées dans le présent document devraient sans doute avoir des effets disproportionnés sur certains pays de l'OCDE.

\section{Structure des flux d'investissement entre la Chine et l'OCDE d'après la nouvelle base de données FTAP sur l'IDE}

12. La nouvelle base de données FTAP sur l'IDE a été construite suivant la procédure décrite par Phamduc (2000). En vue d'assurer la compatibilité avec la base de données GTAP, version 6, employée dans nos simulations, l'année 2001 a été prise pour référence dans la nouvelle base de données FTAP sur l'IDE. Cette base de données a été construite en utilisant les sources d'information suivantes : la base de données de $1^{\prime} \mathrm{OCDE}^{3}$ sur les stocks d'IDE par industrie et par pays partenaire pour tous les pays de l'OCDE, l'annuaire de la CNUCED sur l'investissement dans le monde (World Investment Directory) ${ }^{4}$

2 Lejour et van Leuwen (2005). C'est là la première étude qui ait tenté de s'appuyer sur des données sur les échanges bilatéraux de services dérivées des statistiques incluses dans la base de données GTAP. Les auteurs utilisent les données sur les importations et les exportations totales de services tirées des statistiques de la balance des paiements établies par le FMI, alors que la matrice des échanges bilatéraux est elle issue de la base de données de l'OCDE sur les échanges de services par pays partenaires. Cette étude couvrait 28 pays de l'OCDE (soit environ 75\% des exportations de services répertoriées dans la base de données GTAP). Malgré sa couverture limitée, cette étude représente un grand pas en avant dans le sens d'une amélioration des bases de données utilisées pour modéliser la libéralisation des services.

3 La base de données de l'OCDE sur les stocks d'IDE par industrie et par pays partenaire a été fournie par la Direction des affaires financières et des entreprises (DAF) de l'OCDE.

$4 \quad$ L'annuaire de l'investissement dans le monde (World Investment Directory) de la CNUCED fournit des informations détaillées sur l'investissement direct étranger dans un pays donné, puisqu'il comprend des 
pour la plupart des pays non membres de l'OCDE, les sources des autorités locales pour ce qui est de la Chine, de Hong Kong, Chine, de la Russie, de Singapour, du Chili, du Pérou et du Brésil, et les statistiques de l'investissement direct étranger au sein de l'ANASE (2004) ${ }^{5}$ s'agissant de la Malaisie, des Philippines, de la Thaïlande et du Viet-Nam.

\section{Structure générale des investissements}

13. Au premier abord, l'UE-15 paraît être de toutes les économies ou tous les groupes de pays analysés la principale destinataire d'IDE. Toutefois, les deux-tiers de l'IDE de l'UE-15 sont internes), l'IDE d'un pays de l'UE-15 allant à un autre pays de l'UE-15. De fait, l'UE-15 ne reçoit que 900 milliards USD de l'extérieur, soit environ un tiers de moins que les États-Unis. Au sein de l'UE-15, les principaux destinataires d'IDE sont le Royaume-Uni (7.7\% de l'IDE mondial), l'Allemagne (6.6\%), alors que la Belgique, la France et les Pays-Bas reçoivent chacun plus de 4\% de l'IDE mondial. La région baptisée "reste du monde" se situe au troisième rang des bénéficiaires de stocks d'entrées d'IDE, plusieurs pays recevant des quantités d'IDE comparables (Singapour, Brésil, pays du Moyen-Orient) et les pays d'Amérique latine occupant des places prépondérantes. Hong Kong, Chine, se classe juste derrière, une grande partie de l'IDE dont bénéficie cette économie provenant de Chine (29\%). Mais, de fait, la plupart des flux d'IDE à destination de Hong Kong, Chine, transitent par des paradis fiscaux, les îles vierges britanniques, les Bermudes et les îles Caïmanes comptant pour $42 \%$ de l'IDE reçu par cette économie. Pour finir, la Chine reçoit près de 203 milliards USD, chiffre comparable à celui du Canada, mais deux fois plus élevé que celui de l'IDE vers l'Australie ou le Mexique. Le Japon et la Russie reçoivent chacun 55 milliards USD, la Corée 45 milliards USD, et la Nouvelle-Zélande et la Turquie aux alentours de 20 milliards USD l'une et l'autre (voir le diagramme A3.3). ${ }^{6}$

14. Pour ce qui est des sorties d'IDE, l'UE-15 constitue une fois encore la principale source d'IDE, bien qu'environ $50 \%$ de l'IDE soit là encore interne à l'UE-15, les stocks provenant principalement du Royaume-Uni (11.5\% de l'IDE mondial), des Pays-Bas (10.5\%), de la France et de l'Allemagne (7.5\% l'un et l'autre). Les États-Unis sont le principal partenaire de l'UE-15 (18\% de l'IDE émanant de 1'UE-15). Si l'on s'en tient aux pays considérés individuellement, les États-Unis constituent la principale source d'IDE (17\% de l'IDE mondial), les principaux bénéficiaires des stocks étant le Royaume-Uni (16\% de l'IDE émanant des États-Unis), le Canada (11\%), le Mexique (7\%), ainsi que plusieurs pays de l'UE-15 et d'Amérique latine, alors que la Chine ne reçoit que 1,6\% de l'IDE des États-Unis (voir le diagramme A3.4).

15. Le diagramme A3.5 met clairement en évidence à quel point le secteur des services est un important déterminant des flux d'IDE. Les quatre principaux secteurs du point de vue de l'IDE relèvent tous des services (services aux entreprises, commerce, banque, et autres services) et les secteurs de services reçoivent au total $61 \%$ de l'IDE mondial, contre $31 \%$ pour le secteur manufacturier et $8 \%$ pour le secteur primaire.

statistiques approfondies sur les flux, les stocks et les activités des sociétés multinationales, ventilées par composante, par industrie et par pays investisseur et pays destinataire. Depuis 2002, tous les profils de pays sont mis en ligne sur le site web indiqué ci-après avant d'être publiés sur support papier ou sur CD-Rom : http://www.unctad.org/Templates/Page.asp?intItemID=3198\&lang=1.

5 Voir ANASE (2004) Statistics of Foreign Direct Investment in ASEAN, sixième édition, disponible à l'adresse suivante : http://www.aseansec.org/17215.htm.

6 Voir au tableau A5.1 la correspondance entre les codes sectoriels utilisés aux diagrammes A3.3 à A3.7 et leurs dénominations complètes. Voir au tableau A5.2 la correspondance entre les codes des économies et des groupes de pays utilisés aux diagrammes A3.3 à A3.7 et leurs dénominations complètes. 


\section{Structure des investissements dans le cas de la Chine}

16. Les données sur l'IDE relatives à la Chine sont établies à partir des informations fournies par la CNUCED (203 milliards USD en 2001) et elles ont été ventilées en fonction de la part des différents secteurs dans les entrées d'IDE, compte tenu que l'on ne dispose pas de statistiques ventilées en ce qui concerne les stocks. Hong Kong, Chine, est le principal investisseur en Chine, puisqu'il compte pour $45 \%$ du stock d'entrées d'IDE dont bénéficie cette dernière. La région du "reste du monde" se classe quant à elle au second rang, ce résultat étant principalement imputable au Taïpeï chinois, à Singapour et aux paradis fiscaux (essentiellement les Îles vierges britanniques). Les États-Unis arrivent quant à eux en troisième place des investisseurs en Chine, suivis de près par l'UE-15 et le Japon. Bien que ces trois régions de l'OCDE comptent chacune pour environ $10 \%$ du stock d'entrées d'IDE de la Chine, leurs investissements en Chine ne représentent qu'une faible part de leurs sorties totales d'IDE $(1,7 \%$ pour les États-Unis, 5\% pour le Japon et aux alentours de 1\% pour l'UE-15). (Voir le diagramme A3.6).

17. Il convient de noter que, du point de vue de la répartition sectorielle, bien que la structure économique de la Chine soit plutôt axée sur les industries manufacturières, le secteur des services aux entreprises n'en est pas moins le second bénéficiaire de l'IDE à destination de la Chine $(13 \%$ du stock d'entrées d'IDE de la Chine), légèrement derrière un groupe de secteurs manufacturiers (OMF) composé de diverses industries dont les produits minéraux et les produits en bois et en papier. Le secteur manufacturier ne s'en taille pas moins la part du lion si on le considère dans son ensemble $(63 \%)$, les principaux secteurs étant la métallurgie (MET 12\%), l'industrie électronique (ELE 9\%), l'agroalimentaire (FOOD 9\%) et l'industrie chimique (CHM 8\%).

18. Outre les services aux entreprises, les réseaux de distribution d'électricité (ELY 6\%) et le commerce de gros (TRD 3\%) constituent d'autres importants secteurs de services. L'industrie de la construction, le gaz, l'eau, les services récréatifs, gouvernementaux et sociaux composent le secteur OSV (12\%). La présence étrangère est restreinte dans les secteurs de la communication $(\mathrm{CMN})$ des transports aériens (ATP) et des transports par voie d'eau (WTP) et elle est presque inexistante dans les services financiers (OFI) et les assurances (ISR). (Voir le diagramme A3.7). 
TD/TC/WP(2006)10/FINAL

Tableau A3.1. Importations chinoises en pourcentage des exportations des différents pays, par catégorie de produits

\begin{tabular}{|c|c|c|c|c|c|c|c|c|c|c|c|c|c|c|}
\hline & Australia & $\begin{array}{c}\text { New } \\
\text { Zealand } \\
\end{array}$ & China & $\begin{array}{l}\text { Hong } \\
\text { Kong- } \\
\text { China } \\
\end{array}$ & Russia & Japan & Korea & Canada & $\begin{array}{l}\text { United } \\
\text { States }\end{array}$ & Mexico & EU15 & $\begin{array}{c}\text { Rest of } \\
\text { Western } \\
\text { Europe }\end{array}$ & $\begin{array}{l}\text { Rest of } \\
\text { World }\end{array}$ & $\begin{array}{c}\text { All } \\
\text { countries }\end{array}$ \\
\hline Agriculture and fishing & 15 & 12 & 0 & 11 & 5 & 5 & 6 & 7 & 7 & 0 & 1 & 1 & 5 & 4 \\
\hline Natural resources & 16 & 11 & 0 & 5 & 3 & 43 & 15 & 1 & 3 & 7 & 2 & 1 & 8 & 6 \\
\hline Coal & 0 & 12 & 0 & 0 & 0 & 13 & 0 & 0 & 0 & 0 & 1 & 0 & 0 & 0 \\
\hline Oil & 4 & 3 & 0 & 0 & 1 & 9 & 0 & 0 & 3 & 0 & 1 & 1 & 4 & 3 \\
\hline Food products and beverages & 4 & 4 & 0 & 11 & 7 & 10 & 7 & 2 & 2 & 1 & 1 & 1 & 3 & 2 \\
\hline Textiles, clothing and leather & 8 & 7 & 0 & 24 & 2 & 56 & 27 & 4 & 3 & 0 & 1 & 0 & 4 & 5 \\
\hline Chemicals and chemical & 7 & 5 & 0 & 61 & 14 & 15 & 35 & 3 & 3 & 1 & 1 & 1 & 9 & 5 \\
\hline Other manufacturing & 4 & 11 & 0 & 26 & 10 & 10 & 22 & 1 & 3 & 0 & 1 & 0 & 5 & 3 \\
\hline Metal products & 11 & 2 & 0 & 39 & 9 & 18 & 20 & 1 & 5 & 1 & 2 & 1 & 5 & 5 \\
\hline Motor vehicles and parts & 0 & 0 & 0 & 11 & 1 & 2 & 2 & 0 & 1 & 0 & 1 & 0 & 2 & 1 \\
\hline Machinery and equipment & 5 & 3 & 0 & 51 & 28 & 10 & 12 & 2 & 4 & 0 & 3 & 2 & 6 & 4 \\
\hline Electronic equipment & 5 & 2 & 0 & 33 & 30 & 13 & 12 & 3 & 5 & 2 & 4 & 1 & 7 & 6 \\
\hline Electricity, gas and water & 2 & 0 & 0 & 46 & 0 & 2 & 3 & 0 & 1 & 0 & 0 & 0 & 0 & 1 \\
\hline Construction & 3 & 3 & 0 & 4 & 4 & 5 & 4 & 4 & 4 & 4 & 5 & 4 & 4 & 4 \\
\hline Wholesale trade & 4 & 3 & 0 & 33 & 2 & 11 & 7 & 5 & 3 & 3 & 7 & 6 & 7 & 13 \\
\hline Land transport & 3 & 2 & 0 & 2 & 2 & 2 & 2 & 3 & 3 & 2 & 2 & 2 & 2 & 2 \\
\hline Water transport & 3 & 1 & 0 & 1 & 0 & 0 & 1 & 0 & 3 & 2 & 1 & 1 & 1 & 1 \\
\hline Air transport & 1 & 2 & 0 & 1 & 1 & 0 & 1 & 1 & 2 & 2 & 1 & 1 & 2 & 1 \\
\hline Post and communications & 1 & 1 & 0 & 1 & 1 & 1 & 1 & 1 & 1 & 1 & 1 & 1 & 1 & 1 \\
\hline Finance & 2 & 2 & 0 & 1 & 1 & 1 & 1 & 1 & 1 & 1 & 1 & 1 & 2 & 1 \\
\hline Insurance & 3 & 3 & 0 & 4 & 3 & 1 & 3 & 4 & 4 & 4 & 4 & 4 & 4 & 4 \\
\hline Business activities & 1 & 1 & 0 & 0 & 0 & 1 & 0 & 1 & 1 & 1 & 1 & 1 & 1 & 1 \\
\hline Other services & 2 & 2 & 0 & 2 & 2 & 1 & 2 & 1 & 2 & 2 & 2 & 2 & 2 & 2 \\
\hline Total exports & 7 & 6 & 0 & 23 & 8 & 11 & 16 & 2 & 3 & 1 & 2 & 1 & 5 & 4 \\
\hline Total exports as $\%$ of GDP & 1.5 & 2.2 & 0.0 & 14.1 & 2.7 & 1.2 & 6.7 & 0.7 & 0.3 & 0.2 & 0.6 & 0.5 & 1.7 & 0.9 \\
\hline
\end{tabular}


TD/TC/WP(2006)10/FINAL

Tableau A3.2. Importations de la Chine en pourcentage des importations des différents pays, par catégorie de produits

\begin{tabular}{|c|c|c|c|c|c|c|c|c|c|c|c|c|c|c|}
\hline & Australia & $\begin{array}{c}\mathrm{New} \\
\text { Zealand } \\
\end{array}$ & China & $\begin{array}{l}\text { Hong } \\
\text { Kong- } \\
\text { China } \\
\end{array}$ & Russia & Japan & Korea & Canada & $\begin{array}{l}\text { United } \\
\text { States } \\
\end{array}$ & Mexico & EU15 & $\begin{array}{c}\text { Rest of } \\
\text { Western } \\
\text { Europe } \\
\end{array}$ & $\begin{array}{l}\text { Rest of } \\
\text { World }\end{array}$ & $\begin{array}{c}\text { All } \\
\text { countries } \\
\end{array}$ \\
\hline Agriculture and fishing & 6 & 3 & 0 & 25 & 6 & 12 & 18 & 2 & 3 & 1 & 2 & 2 & 5 & 4 \\
\hline Natural resources & 10 & 19 & 0 & 26 & 0 & 2 & 2 & 1 & 1 & 1 & 1 & 0 & 2 & 1 \\
\hline Coal & 0 & 0 & 0 & 44 & 3 & 17 & 41 & 2 & 4 & 4 & 3 & 1 & 17 & 14 \\
\hline Oil & 1 & 0 & 0 & 0 & 1 & 1 & 0 & 0 & 0 & 7 & 0 & 0 & 1 & 0 \\
\hline Food products and beverages & 4 & 2 & 0 & 22 & 2 & 13 & 15 & 1 & 2 & 0 & 1 & 1 & 2 & 3 \\
\hline Textiles, clothing and leather & 49 & 38 & 0 & 74 & 31 & 65 & 45 & 21 & 21 & 5 & 9 & 10 & 17 & 21 \\
\hline Chemicals and chemical products & 7 & 3 & 0 & 23 & 2 & 8 & 8 & 2 & 5 & 1 & 2 & 1 & 5 & 3 \\
\hline Other manufacturing & 15 & 10 & 0 & 51 & 7 & 24 & 15 & 9 & 23 & 6 & 6 & 3 & 6 & 12 \\
\hline Metal products & 9 & 4 & 0 & 26 & 1 & 12 & 9 & 3 & 8 & 1 & 2 & 1 & 5 & 4 \\
\hline Motor vehicles and parts & 0 & 0 & 0 & 7 & 0 & 4 & 14 & 0 & 0 & 0 & 0 & 0 & 1 & 1 \\
\hline Machinery and equipment & 5 & 3 & 0 & 40 & 2 & 18 & 6 & 3 & 9 & 2 & 3 & 2 & 5 & 5 \\
\hline Electronic equipment & 9 & 9 & 0 & 33 & 4 & 16 & 10 & 7 & 12 & 4 & 5 & 5 & 6 & 8 \\
\hline Electricity, gas and water & 3 & 3 & 0 & 46 & 1 & 1 & 1 & 0 & 1 & 1 & 0 & 0 & 0 & 1 \\
\hline Construction & 1 & 2 & 0 & 3 & 2 & 3 & 1 & 2 & 1 & 1 & 3 & 2 & 3 & 3 \\
\hline Wholesale trade & 3 & 3 & 0 & 7 & 3 & 6 & 4 & 3 & 2 & 5 & 7 & 5 & 5 & 5 \\
\hline Land transport & 3 & 3 & 0 & 3 & 3 & 3 & 2 & 3 & 5 & 3 & 3 & 2 & 3 & 3 \\
\hline Water transport & 1 & 1 & 0 & 1 & 2 & 0 & 0 & 2 & 4 & 5 & 1 & 0 & 1 & 1 \\
\hline Air transport & 1 & 1 & 0 & 1 & 1 & 1 & 0 & 1 & 1 & 1 & 1 & 1 & 1 & 1 \\
\hline Post and communications & 1 & 1 & 0 & 1 & 2 & 1 & 1 & 1 & 1 & 1 & 1 & 1 & 2 & 1 \\
\hline Finance & 2 & 2 & 0 & 1 & 4 & 1 & 2 & 1 & 2 & 1 & 1 & 1 & 2 & 1 \\
\hline Insurance & 1 & 1 & 0 & 1 & 1 & 1 & 1 & 1 & 1 & 1 & 1 & 1 & 1 & 1 \\
\hline Business activities & 1 & 1 & 0 & 1 & 1 & 1 & 0 & 1 & 1 & 1 & 1 & 1 & 1 & 1 \\
\hline Other services & 2 & 2 & 0 & 1 & 2 & 2 & 2 & 1 & 2 & 2 & 2 & 2 & 1 & 2 \\
\hline Total imports & 8 & 5 & 0 & 35 & 5 & 14 & 9 & 4 & 9 & 2 & 3 & 2 & 5 & 6 \\
\hline Total imports as $\%$ of GDP & 1.6 & 1.6 & 0.0 & 23.7 & 1.1 & 1.4 & 3.3 & 1.2 & 1.1 & 0.5 & 0.9 & 0.9 & 1.4 & 1.2 \\
\hline
\end{tabular}


TD/TC/WP(2006)10/FINAL

\section{Diagramme A3.1. Structure des importations chinoises}

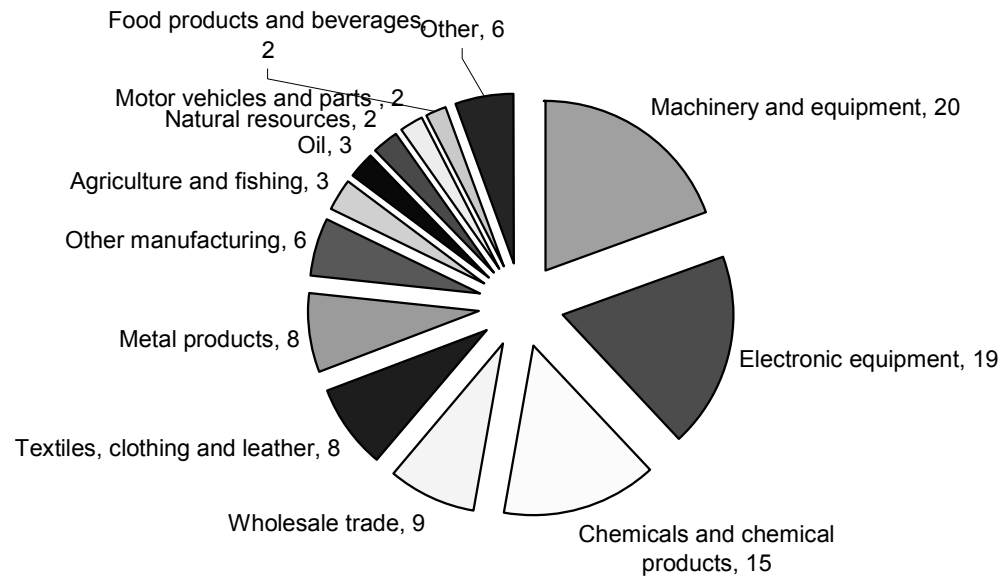

Source : GTAP version 6. 


\section{Diagramme A3.2. Structure des exportations de la Chine}

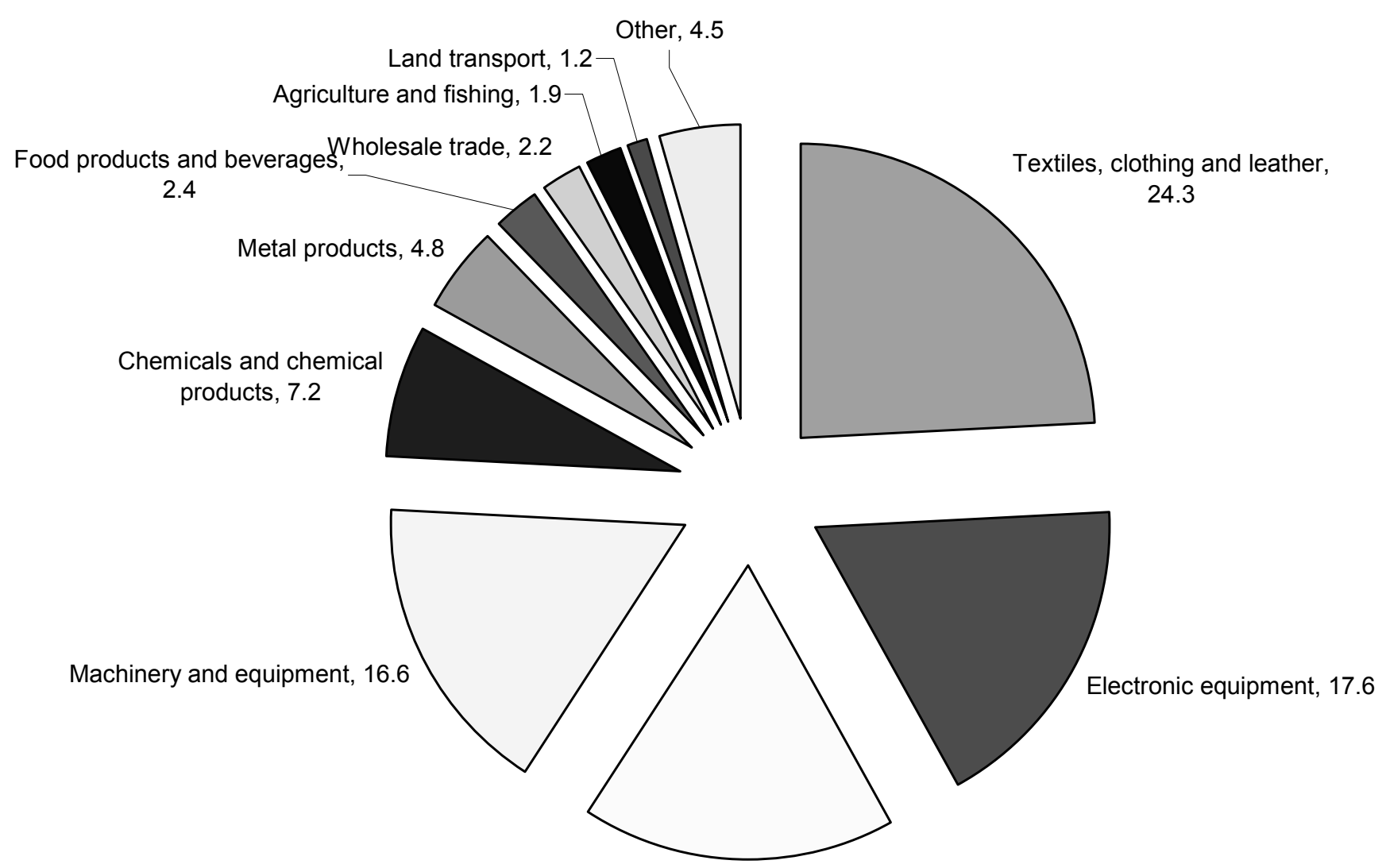

Other manufacturing, 17. 
Tableau A3.3. Part des différents pays dans les importations de la Chine

\begin{tabular}{ccccccccc}
\hline Australia & $\begin{array}{c}\text { New } \\
\text { Zealand }\end{array}$ & Japan & Korea & Canada & USA & Mexico & EU15 & $\begin{array}{c}\text { Rest of } \\
\text { Europe }\end{array}$ \\
\hline 15 & 3 & 1 & 1 & 9 & 26 & 0 & 4 & 1 \\
18 & 1 & 1 & 0 & 3 & 2 & 1 & 4 & 1 \\
39 & 13 & 0 & 0 & 1 & 10 & 0 & 0 & 1 \\
1 & 0 & 0 & 0 & 0 & 0 & 0 & 1 & 2 \\
7 & 5 & 4 & 2 & 4 & 13 & 1 & 13 & 3 \\
0 & 0 & 26 & 24 & 1 & 3 & 0 & 7 & 0 \\
1 & 0 & 16 & 20 & 2 & 9 & 0 & 10 & 1 \\
1 & 2 & 13 & 11 & 4 & 12 & 0 & 17 & 1 \\
6 & 0 & 24 & 12 & 1 & 8 & 0 & 12 & 2 \\
0 & 0 & 30 & 5 & 3 & 5 & 0 & 47 & 2 \\
0 & 0 & 26 & 7 & 1 & 16 & 0 & 29 & 3 \\
0 & 0 & 25 & 12 & 1 & 11 & 1 & 15 & 0 \\
1 & 0 & 0 & 0 & 2 & 9 & 0 & 26 & 9 \\
0 & 0 & 17 & 0 & 1 & 9 & 1 & 55 & 5 \\
0 & 0 & 3 & 0 & 0 & 2 & 0 & 18 & 2 \\
1 & 0 & 1 & 1 & 4 & 22 & 2 & 33 & 9 \\
3 & 1 & 1 & 2 & 2 & 18 & 1 & 34 & 8 \\
3 & 1 & 2 & 1 & 4 & 25 & 2 & 29 & 4 \\
2 & 1 & 2 & 2 & 3 & 20 & 1 & 39 & 6 \\
1 & 0 & 2 & 1 & 1 & 32 & 1 & 36 & 9 \\
1 & 0 & 0 & 0 & 7 & 12 & 4 & 50 & 6 \\
1 & 0 & 2 & 1 & 2 & 16 & 0 & 47 & 4 \\
2 & 0 & 1 & 2 & 1 & 38 & 1 & 35 & 5 \\
2 & 0 & 18 & 10 & 2 & 11 & 0 & 17 & 2 \\
\hline
\end{tabular}

All products 
TD/TC/WP(2006)10/FINAL

Tableau A3.4. Indices bilatéraux des échanges intra-branche avec la Chine

\begin{tabular}{|c|c|c|c|c|c|c|c|c|c|c|c|c|c|c|}
\hline & Australia & $\begin{array}{c}\text { New } \\
\text { Zealand } \\
\end{array}$ & China & $\begin{array}{l}\text { Hong Kong- } \\
\text { China } \\
\end{array}$ & Russia & Japan & Korea & Canada & $\begin{array}{c}\text { United } \\
\text { States } \\
\end{array}$ & Mexico & EU15 & $\begin{array}{c}\text { Rest of } \\
\text { Western } \\
\text { Europe } \\
\end{array}$ & $\begin{array}{c}\text { Rest of } \\
\text { World } \\
\end{array}$ & $\begin{array}{l}\text { coeff. of } \\
\text { variation } \\
\end{array}$ \\
\hline Agriculture and fishing & 0.1 & 0.1 & - & 0.0 & 0.5 & 0.1 & 0.0 & 0.2 & 0.3 & 0.1 & 0.5 & 0.5 & 0.6 & 0.9 \\
\hline Natural resources & 0.0 & 0.4 & - & 0.0 & 0.0 & 0.4 & 0.1 & 0.1 & 0.8 & 0.3 & 0.8 & 0.5 & 0.2 & 0.9 \\
\hline Coal & 0.0 & 0.0 & - & 0.0 & 0.2 & 0.0 & 0.0 & 0.1 & 0.5 & 0.0 & 0.0 & 0.1 & 0.1 & 1.9 \\
\hline Oil & 0.3 & 0.7 & - & - & 0.0 & 0.0 & 0.0 & 1.0 & 0.0 & 0.0 & 1.0 & 0.1 & 0.1 & 1.4 \\
\hline Food products and beverages & 0.4 & 0.1 & - & 0.1 & 0.8 & 0.1 & 0.3 & 0.7 & 1.0 & 0.8 & 0.9 & 1.0 & 0.8 & 0.6 \\
\hline Textiles, clothing and leather & 0.1 & 0.2 & - & 0.3 & 0.0 & 0.5 & 0.7 & 0.1 & 0.1 & 0.1 & 0.2 & 0.1 & 0.6 & 0.9 \\
\hline Chemicals and chemical products & 0.6 & 1.0 & - & 0.5 & 0.1 & 0.6 & 0.3 & 0.8 & 0.8 & 0.3 & 0.9 & 0.9 & 0.8 & 0.4 \\
\hline Other manufacturing & 0.2 & 0.7 & - & 0.2 & 0.8 & 0.5 & 0.8 & 0.5 & 0.1 & 0.0 & 0.3 & 0.2 & 0.9 & 0.7 \\
\hline Metal products & 0.4 & 0.9 & - & 0.5 & 0.1 & 0.6 & 0.6 & 0.4 & 0.5 & 0.7 & 0.9 & 1.0 & 1.0 & 0.4 \\
\hline Motor vehicles and parts & 0.4 & 0.3 & - & 0.0 & 0.5 & 0.2 & 1.0 & 0.4 & 0.7 & 0.8 & 0.1 & 0.4 & 0.8 & 0.6 \\
\hline Machinery and equipment & 0.4 & 0.5 & - & 0.5 & 0.2 & 0.7 & 0.6 & 0.7 & 0.6 & 0.2 & 0.9 & 1.0 & 0.7 & 0.4 \\
\hline Electronic equipment & 0.2 & 0.1 & - & 0.2 & 0.8 & 0.8 & 0.6 & 0.5 & 0.5 & 0.7 & 0.8 & 0.3 & 0.8 & 0.5 \\
\hline Electricity, gas and water & 0.6 & 0.0 & - & 0.4 & 0.6 & 0.1 & 0.8 & 1.0 & 1.0 & 0.3 & 0.9 & 0.6 & 0.9 & 0.6 \\
\hline Construction & 0.2 & 0.7 & - & 0.6 & 0.6 & 0.8 & 0.3 & 0.5 & 0.2 & 0.0 & 0.7 & 0.6 & 1.0 & 0.6 \\
\hline Wholesale trade & 0.8 & 0.7 & - & 0.0 & 0.0 & 0.9 & 1.0 & 0.8 & 1.0 & 0.4 & 1.0 & 0.8 & 0.9 & 0.5 \\
\hline Land transport & 0.8 & 0.5 & - & 0.6 & 0.4 & 0.3 & 0.8 & 0.9 & 0.7 & 0.7 & 0.8 & 0.8 & 0.9 & 0.3 \\
\hline Water transport & 0.8 & 0.8 & - & 0.6 & 0.2 & 0.2 & 0.9 & 0.7 & 0.9 & 0.9 & 0.8 & 0.9 & 1.0 & 0.4 \\
\hline Air transport & 0.7 & 0.4 & - & 0.4 & 0.5 & 0.4 & 0.9 & 0.7 & 1.0 & 0.6 & 0.9 & 0.7 & 0.7 & 0.3 \\
\hline Post and communications & 0.9 & 0.8 & - & 0.7 & 0.7 & 0.5 & 0.9 & 0.9 & 1.0 & 0.9 & 0.9 & 1.0 & 0.9 & 0.2 \\
\hline Finance & 0.9 & 0.8 & - & 0.9 & 0.1 & 0.4 & 0.8 & 0.4 & 0.8 & 0.6 & 0.8 & 0.8 & 0.8 & 0.4 \\
\hline Insurance & 0.2 & 0.5 & - & 0.5 & 0.8 & 0.4 & 0.3 & 0.3 & 0.3 & 0.7 & 0.2 & 0.2 & 0.5 & 0.5 \\
\hline Business activities & 1.0 & 1.0 & - & 0.9 & 0.3 & 0.6 & 0.7 & 1.0 & 0.8 & 0.9 & 0.9 & 0.8 & 1.0 & 0.2 \\
\hline Other services & 0.8 & 0.8 & - & 0.5 & 0.6 & 0.3 & 0.9 & 0.7 & 0.7 & 0.8 & 1.0 & 0.8 & 0.9 & 0.3 \\
\hline coeff. of variation & 0.7 & 0.6 & - & 0.7 & 0.7 & 0.6 & 0.6 & 0.5 & 0.5 & 0.7 & 0.4 & 0.5 & 0.4 & \\
\hline
\end{tabular}


TD/TC/WP(2006)10/FINAL

Tableau A3.5. Indices des échanges intra-branche par produit et par pays partenaire

\begin{tabular}{ll|lc}
\hline by sector & & by country & \\
\hline Agriculture and fishing & 0.3 & Australia & 0.3 \\
Natural resources & 0.2 & New Zealand & 0.4 \\
Coal & 0.0 & China & - \\
Oil & 0.1 & Hong Kong-China & 0.3 \\
Food products and beverages & 0.5 & Russia & 0.2 \\
Textiles, clothing and leather & 0.3 & Japan & 0.6 \\
Chemicals and chemical products & 0.7 & Korea & 0.5 \\
Other manufacturing & 0.4 & Canada & 0.5 \\
Metal products & 0.7 & United States & 0.4 \\
Motor vehicles and parts & 0.4 & Mexico & 0.4 \\
Machinery and equipment & 0.7 & EU15 & 0.7 \\
Electronic equipment & 0.6 & Rest of Western Europe & 0.6 \\
Electricity, gas and water & 0.6 & Rest of World & 0.7 \\
Construction & 0.7 & & \\
Wholesale trade & 0.5 & & \\
Land transport & 0.8 & & \\
Water transport & 0.8 & & \\
Air transport & 0.8 & & \\
Post and communications & 0.9 & & \\
Finance & 0.8 & & \\
Insurance & 0.3 & & \\
Business activities & 0.9 & & \\
Other services & 0.8 & & \\
\hline Source: calculs des auteurs à partir de la base de données GTAP, version 6. & \\
& & & \\
& &
\end{tabular}


Diagramme A3.3. Destination de I'IDE, en \% de I'IDE mondial

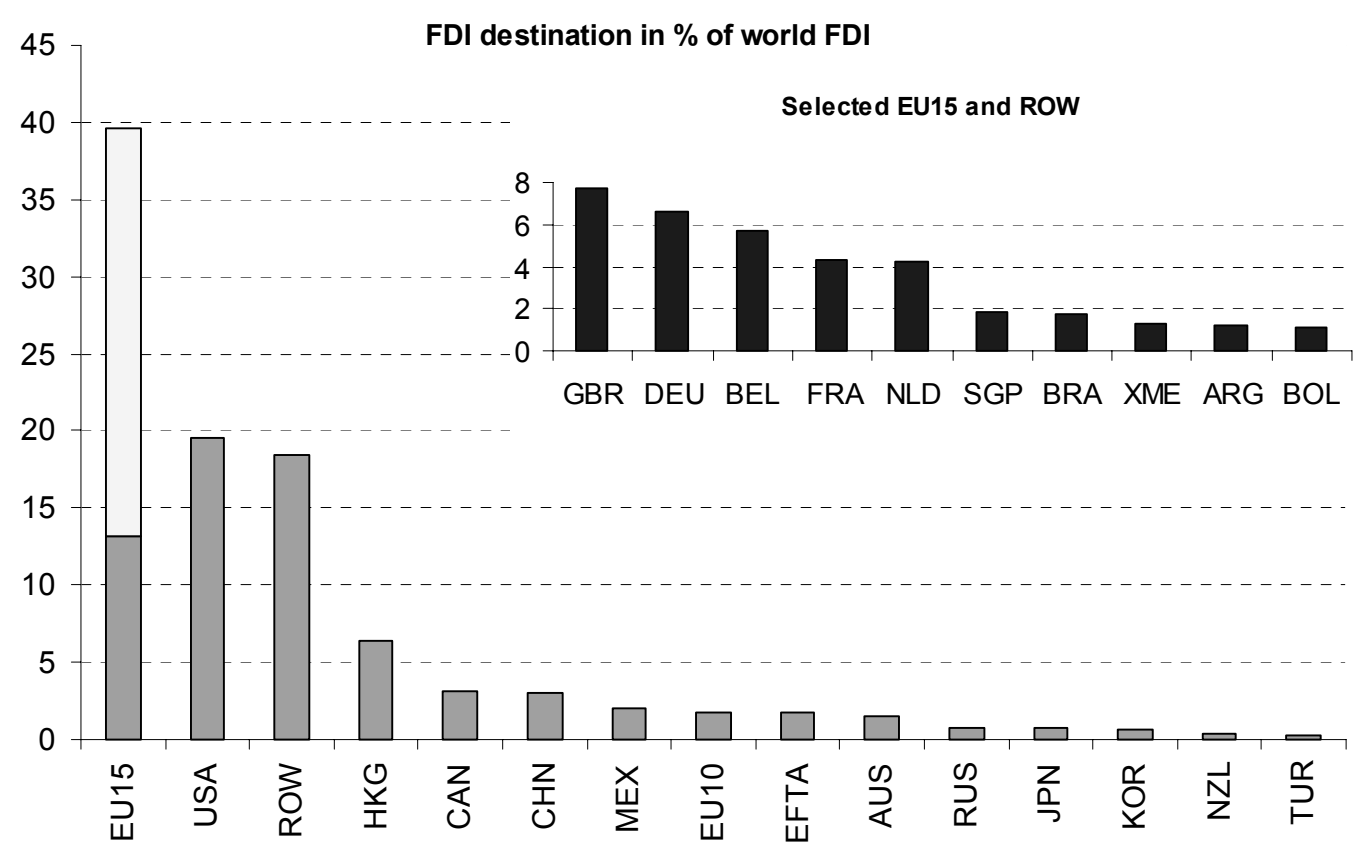


TD/TC/WP(2006)10/FINAL

Diagramme A3.4. Provenance de l'IDE, en \% de l'IDE mondial

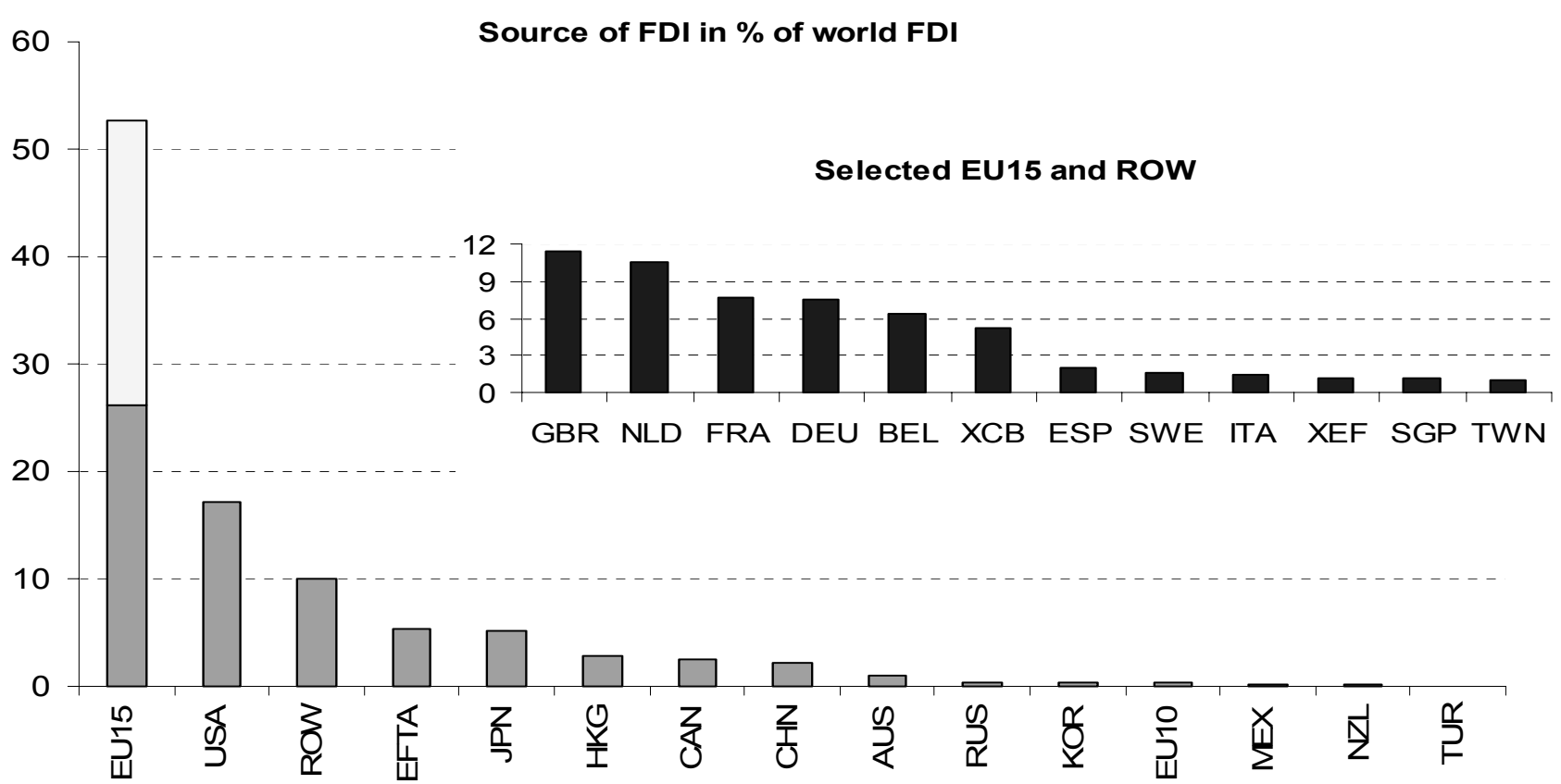

Source : calculs des auteurs (base de données FTAP 2001). 
Diagramme A3.5. IDE mondial par branche d'activité, en \% de l'IDE mondial

World FDI by industry, in \% of world FDI (US\$6893bn)

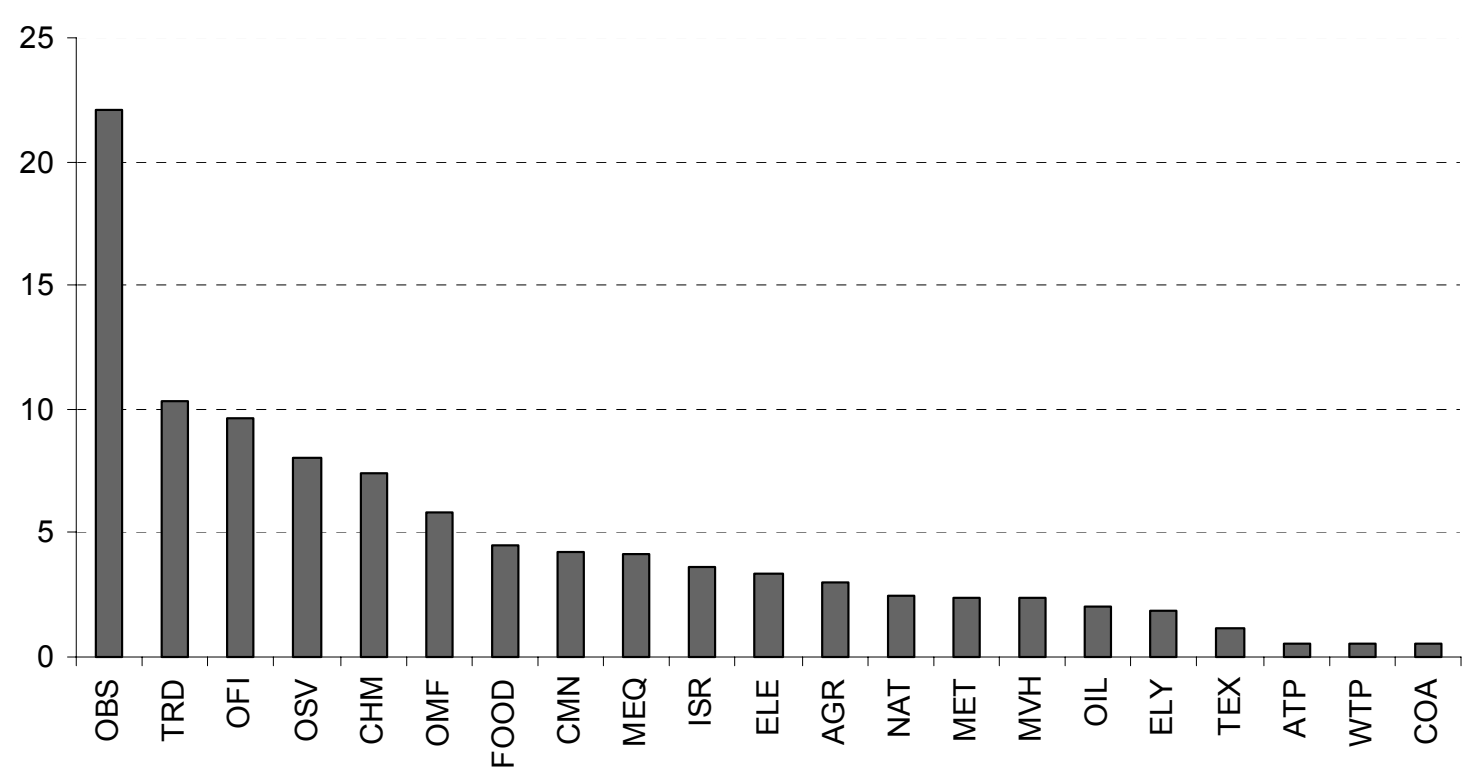

Source : calculs des auteurs (base de données FTAP 2001). 
FDI to CHN in \% of overall inward stock (203142 million US\$)

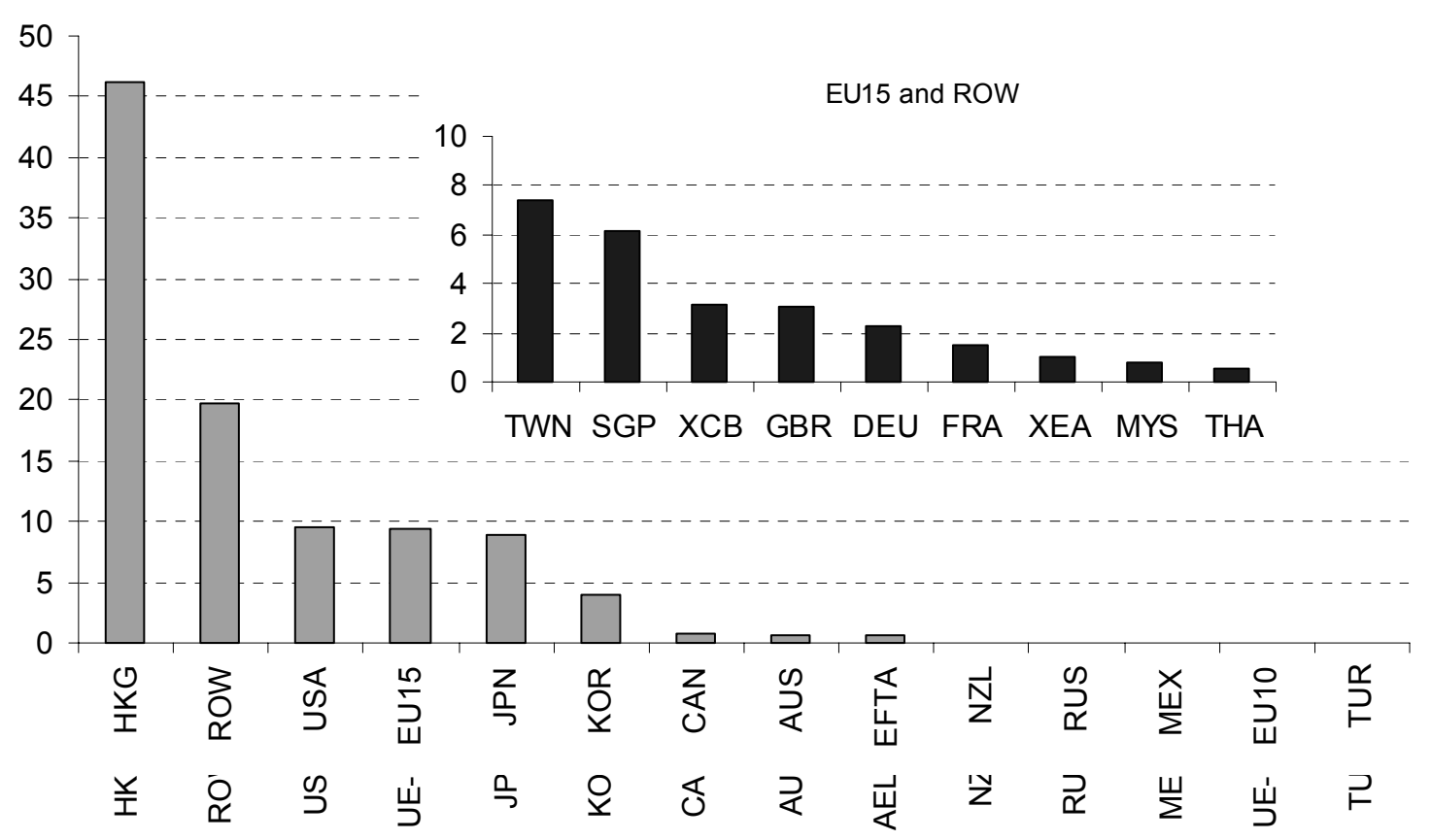


TD/TC/WP(2006)10/FINAL

Diagramme A3.7. IDE à destination de la Chine par branche d'activité, en \% du stock total d'entrées d'IDE

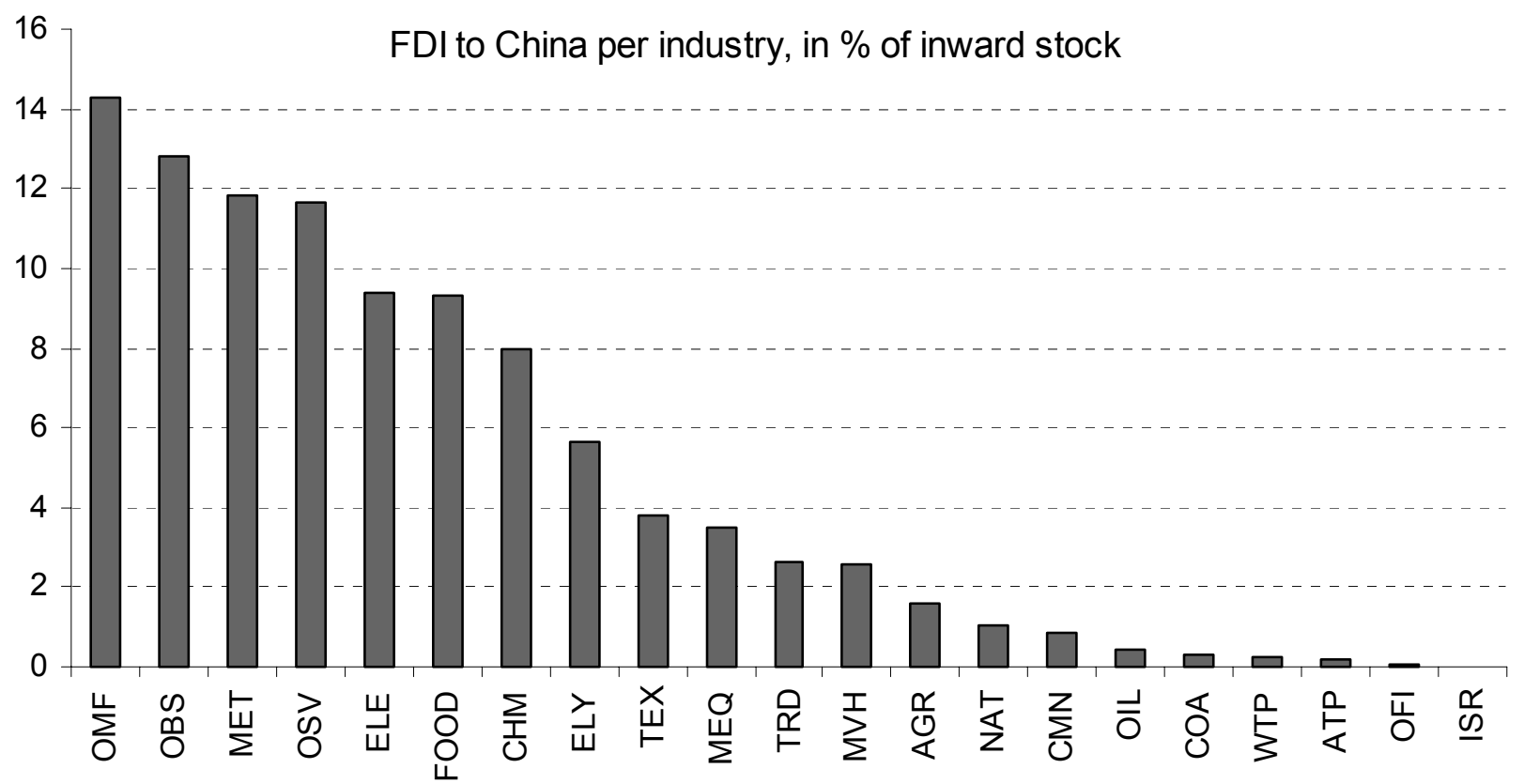

Source : calculs des auteurs (base de données FTAP 2001). 


\section{ANNEXE 4 : ÉCONOMIE DU MODÈLE FTAP}

19. La présente annexe offre une brève description du modèle FTAP d'analyse de l'investissement direct étranger et des échanges utilisé dans le cadre de l'exercice de modélisation présenté à la section III de ce document. Le modèle FTAP modélise les activités de trois "agents" économiques dans chaque région : les entreprises, l'administration publique et un ménage représentatif (autrement dit les particuliers). Il s'inspire du cadre d'analyse du modèle GTAP standard de Hertel (1997) pour décrire la localisation de l'activité économique, qui détermine la provenance des produits, avant de procéder à une désagrégation des données en fonction de l'origine du capital des entreprises. Chaque industrie opérant en Chine comprend ainsi aussi bien des entreprises chinoises que des entreprises multinationales originaires de différents pays. Chacune de ces catégories d'entreprises, définies en fonction de l'origine de leur capital, est supposée faire en toute indépendance ses choix concernant les intrants de production, conformément à la théorie du modèle GTAP standard. Chaque catégorie d'entreprises se caractérise en outre par une structure des ventes qui lui est propre.

20. Pour ce qui est des achats, les agents font leur choix parmi les produits ou les services proposés par les différentes catégories d'entreprises, qui se distinguent aussi bien par l'origine du capital que par la localisation, puis parmi ceux proposés par les différentes entreprises (symétriques) d'une même catégorie - le modèle tient compte de la différenciation des produits au niveau de l'entreprise qui est associée à une concurrence monopolistique. Les entreprises choisissent parmi les intrants intermédiaires et les biens de production, alors que les ménages et les administrations publiques font leur choix parmi les biens et services finals.

21. Les agents sont supposés choisir d'abord entre les produits ou services selon qu'ils sont de provenance nationale ou étrangère, avec une élasticité constante de substitution égale à 5 . Ils font alors leur choix parmi certaines provenances étrangères, puis entre les différentes catégories d'origine du capital des entreprises pour une provenance donnée, avec une élasticité constante de substitution égale à 10 dans l'un et l'autre cas. Ils font enfin leur choix parmi les différentes entreprises d'une même catégorie, définie en fonction de l'origine du capital et de la localisation, avec une élasticité constante de substitution égale à 15 . Du fait de la différenciation des produits au niveau de l'entreprise, les agents ont la possibilité de choisir entre un plus grand nombre d'entreprises et bénéficient donc d'une plus grande probabilité de trouver un produit ou un service susceptibles de répondre à leurs besoins particuliers ${ }^{122}$ (voir la figure A4.1).

\footnotetext{
122 Les deux premiers choix, entre les provenances nationale et étrangère, sont identiques à ceux proposés dans le modèle GTAP de départ. Ils ont été paramétrés à l'aide de valeurs, 5 et 10, environ deux fois supérieures aux élasticités d'Armington du GTAP standard. Deux raisons peuvent être invoquées pour justifier cette multiplication par deux des élasticités du modèle standard. Tout d'abord, le modèle GTAP ne peut parvenir à reproduire les évolutions passées de la structure des échanges qu'avec ces élasticités (Gehlhar, 1997). En second lieu, ces élasticités plus élevées sont plus en accord avec l'idée d'une différenciation des produits au niveau de l'entreprise.
} 
Figure A4.1. Structure de la demande dans le modèle FTAP

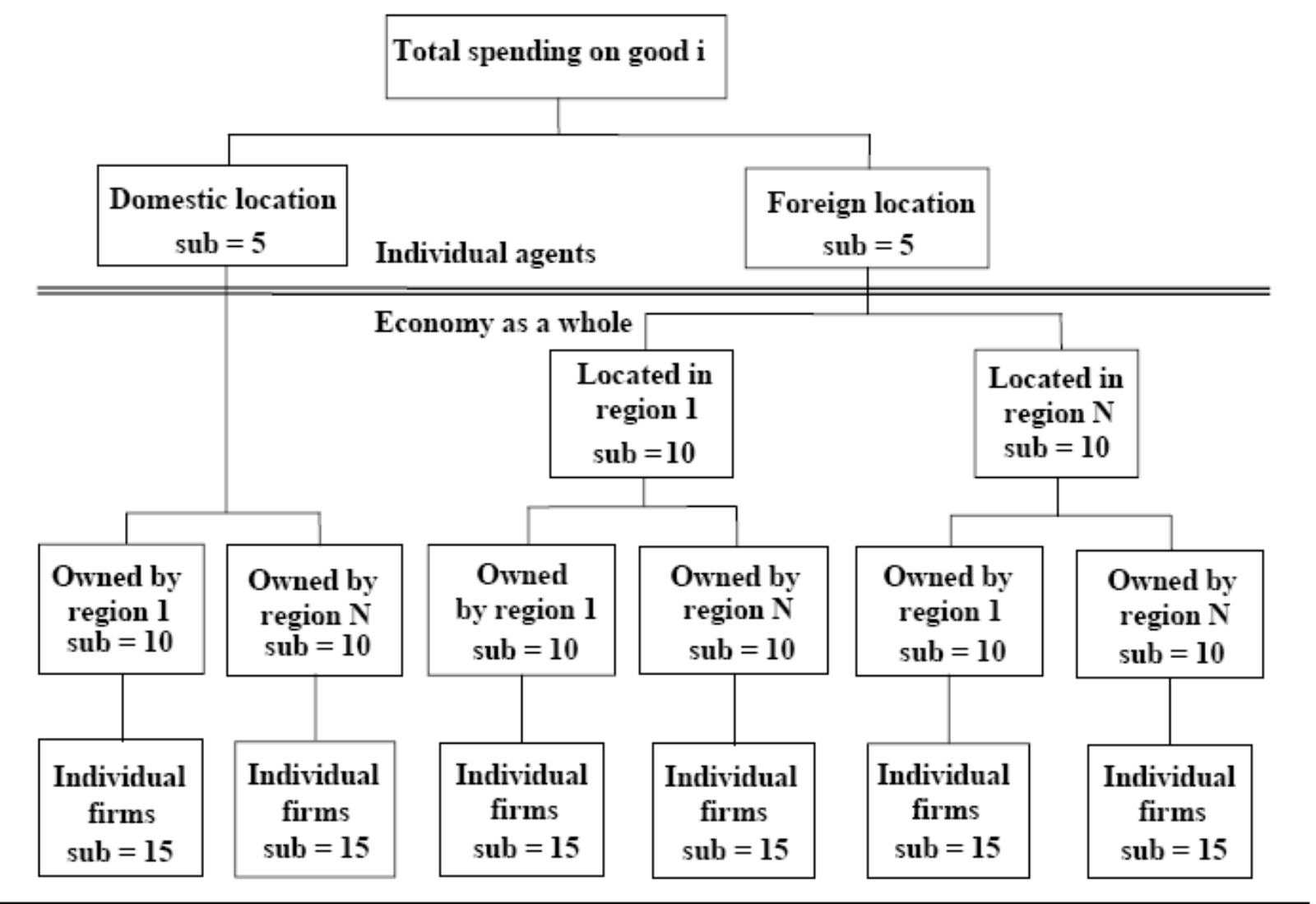

Source : Hanslow et al. (1999), "The Structure of the FTAP model".

22. Pour ce qui est de la demande, chacune des régions d'accueil possède deux types d'entreprises dans chaque secteur : des entreprises nationales (maison mère de la multinationale locale) et diverses filiales de sociétés étrangères. Les entreprises peuvent être classées en fonction de leur localisation (secteur de la région d'accueil) et de l'origine de leur capital (région d'origine). Les filiales étrangères combinent les capitaux provenant de leur région d'origine à la main-d'œuvre, aux terres et aux ressources naturelles de la région d'accueil pour produire des biens et des services pour les consommateurs de la région d'accueil et pour l'exportation. Dans chacun des secteurs, les produits des entreprises nationales et ceux des filiales de sociétés étrangères sont différenciés.

23. Les consommateurs de la région d'accueil sont supposés répartir une part constante de leurs revenus nominaux entre les différents biens et services. L'utilité des consommateurs est supposée être fonction croissante du nombre de variétés disponibles d'un même produit, ainsi que de l'éventail de produits différents qui leur sont proposés. La fourniture des biens et services produits à l'étranger peut être assurée au moyen d'échanges transfrontières ou par des filiales étrangères présentes dans la région d'accueil. La plupart des biens et services importés sont supposés être imparfaitement substituables à leurs équivalents produits au sein de la région considérée.

24. Compte tenu de ces caractéristiques, une augmentation du volume de la demande de services satisfaite par les filiales de sociétés étrangères dans une région d'accueil se traduit par une présence commerciale accrue de ces filiales. Une telle augmentation du volume de la demande peut être la 
conséquence d'une baisse du prix des produits et des coûts de production (c'est-à-dire en d'autres termes d'une augmentation de l'offre).

25. Pour ce qui est de l'offre, le ménage représentatif est supposé détenir tous les facteurs de production primaires : terres, ressources naturelles, capital et travail. L'offre de terres, de ressources naturelles et de travail sont considérées comme données dans chaque région. Les terres et les ressources naturelles ne sont utilisées que par les industries primaires, et ce en quantités constantes. Le facteur travail, ou en d'autres termes la main-d'œuvre, se caractérise par sa mobilité intersectorielle au sein de chaque région, mais pas entre les régions. Le capital se caractérise pour sa part par sa mobilité intersectorielle au sein de chaque région comme entre les régions. S'agissant de l'allocation du capital entre les différentes régions et les différents secteurs, le modèle FTAP se conforme au principe de transformation imparfaite des différents types d'actifs formulé par Petri (1997).

Figure A4.2. Fonction de l'offre d'actifs dans le modèle FTAP

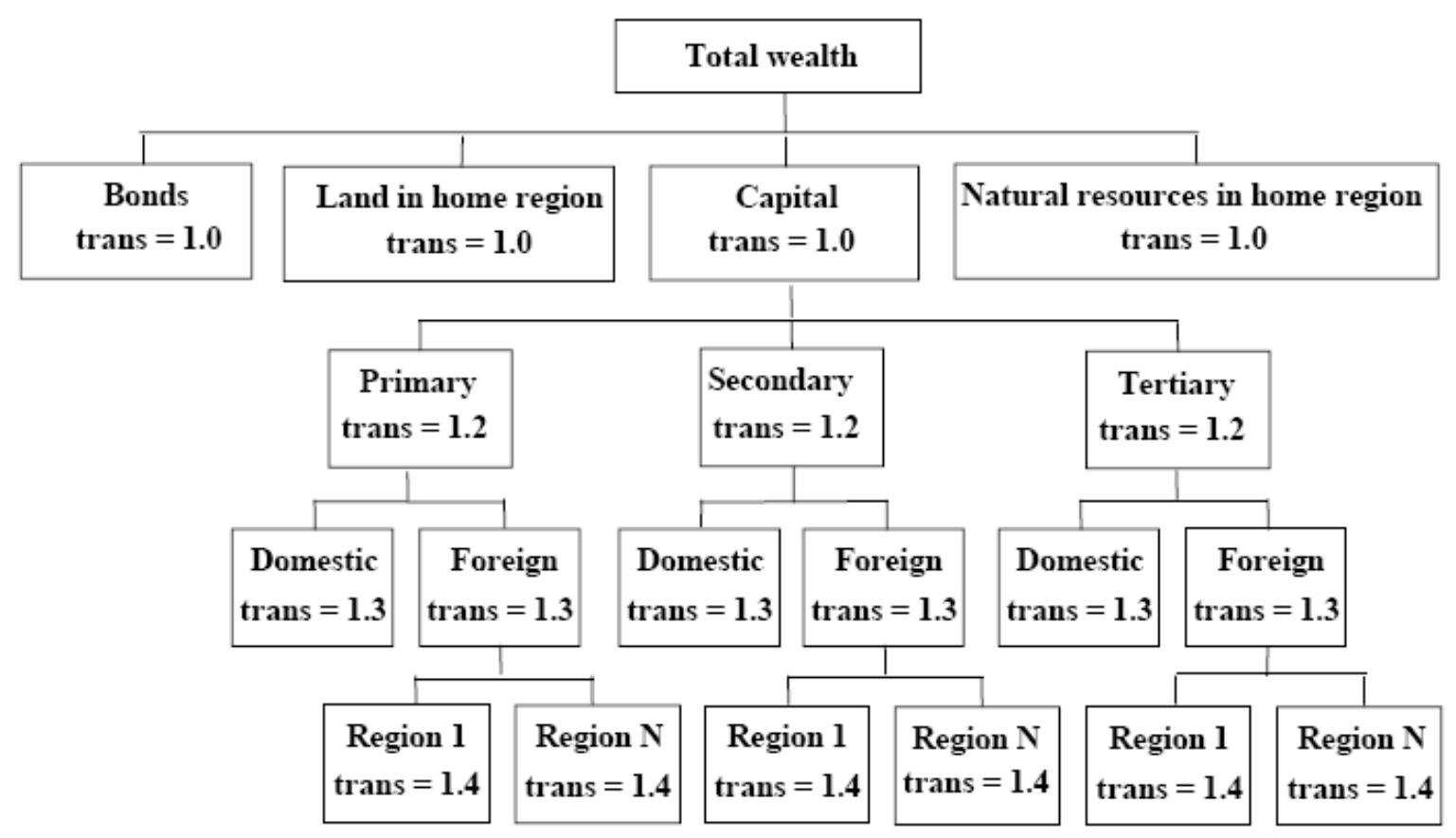

Source : Hanslow et al. (1999), "The Structure of the FTAP model".

26. Les investisseurs au sein de chaque économie répartissent tout d'abord leurs actifs entre les "titres" (qui peuvent consister en n'importe quel instrument d'investissement de portefeuille), le capital physique réel et les terres et les ressources naturelles dans le pays d'accueil. Ce choix est régi par une semi-élasticité constante de transformation égale à 1 , ce qui signifie par exemple que un point de pourcentage d'augmentation du taux de rendement du capital physique réel accroîtrait de $1 \%$ le rapport entre le volume du capital physique réel et celui du portefeuille de titres détenus. Un titre est un titre, quel qu'en soit l'émetteur, ce qui implique un arbitrage international parfait entre les taux de rendement des différents titres. Le capital est cependant différencié selon les localisations. Les investisseurs choisissent ensuite le secteur d'activité dans lequel ils investissent (avec une semi-élasticité constante de transformation égale à 1.2). Puis ils décident s'ils veulent investir dans leur région ou à l'étranger dans le secteur retenu par eux (avec une semi-élasticité constante de transformation égale à 1.3). Pour finir, ils choisissent une région précise à l'étranger au sein de laquelle investir (avec une semi-élasticité constante de transformation égale à 1.4). 
27. On distingue deux niveaux d'investissement direct. L'entreprise multinationale opérant dans un secteur donné prend la décision d'investir dans les activités qu'elle mène dans un secteur donné dans les différentes régions, alors que l'investisseur régional prend la décision d'investir dans les entreprises de la région quel que soit leur secteur d'activité. L'investisseur régional ne peut investir son capital à l'étranger qu'en l'investissant dans les entreprises multinationales de la région.

28. L'un et l'autre niveau d'investissement étranger obéissent au même principe de maximisation de leurs rendements. Au niveau de l'entreprise, une société multinationale ne peut maximiser le rendement des investissements que si la maison mère dans la région d'origine et toutes ses filiales à l'étranger génèrent un même taux de rendement pour chaque unité de capital utilisé. Au niveau régional, le rendement du capital régional total ne peut être maximisé que si l'ensemble des entreprises multinationales d'origine locale génèrent, quel que soit leur secteur d'activité, un même taux de rendement pour chaque unité de capital utilisé. 


\title{
ANNEXE 5 : RÉSULTATS DE LA MODÉLISATION
}

\author{
ANNEXE TABLEAUX ET FIGURES
}

Tableau A5.1. Groupes de produits définis par référence à la classification sectorielle du GTAP

\begin{tabular}{|c|c|c|c|}
\hline Product group & Product code & GTAP sectoral classification & GTAP code \\
\hline Agriculture and fishing & AGR & $\begin{array}{l}\text { Paddy rice, Wheat, Cereal grains nec, Vegetables and fruits, } \\
\text { Oil seeds Sugar cane sugar beet, Plant-based fibers, Crops } \\
\text { nec, Cattle sheep goats horses, Animal products nec, Raw } \\
\text { milk, Wool silk-worm cocoons, Fishing }\end{array}$ & $\begin{array}{l}\text { pdr, wht, gro, v_f, } \\
\text { nuts,osd, c_b, pfb } \\
\text { ocr, ctl, oap, rmk } \\
\text { wol, fsh }\end{array}$ \\
\hline Natural resources & NAT & Forestry, Gas, Minerals nec & frs, gas, omn \\
\hline Coal & $\operatorname{COA}$ & Coal & coa \\
\hline Oil & OIL & Oil & oil \\
\hline $\begin{array}{l}\text { Food products and } \\
\text { beverages }\end{array}$ & FOOD & $\begin{array}{l}\text { Meat: cattle sheep goats horse, Meat products nec, } \\
\text { Vegetable oils and fats, Dairy products, Processed rice, } \\
\text { Sugar, Food products nec, Beverages and tobacco } \\
\text { products }\end{array}$ & $\begin{array}{l}\text { Cmt, omt, vol, mil, } \\
\text { pcr, sgr, ofd } \\
\text { b_t }\end{array}$ \\
\hline $\begin{array}{l}\text { Textiles, clothing and } \\
\text { leather }\end{array}$ & TEX & Textiles, Wearing apparel, Leather products & tex, wap lea \\
\hline $\begin{array}{l}\text { Chemicals and } \\
\text { chemical products }\end{array}$ & $\mathrm{CHM}$ & Petroleum coal products, Chemical rubber plastic prods & p_c, crp \\
\hline $\begin{array}{l}\text { Chemicals and } \\
\text { chemical products }\end{array}$ & OMF & $\begin{array}{l}\text { Wood products, Paper products publishing } \\
\text { Mineral products nec } \\
\text { Manufactures nec }\end{array}$ & $\begin{array}{l}\text { lum, ppp,nmm, } \\
\text { omf }\end{array}$ \\
\hline Metal products & MET & Ferrous metals, Metals nec, Metal products & i_s, nfm, fmp \\
\hline Motor vehicles & MVH & Motor vehicles and parts & $\mathrm{mvh}$ \\
\hline $\begin{array}{l}\text { Machinery and } \\
\text { equipment }\end{array}$ & MEQ & $\begin{array}{l}\text { Transport equipment nec } \\
\text { Machinery and equipment nec }\end{array}$ & $\begin{array}{l}\text { otn } \\
\text { ome }\end{array}$ \\
\hline Electronic equipment & ELE & Electronic equipment & ele \\
\hline $\begin{array}{l}\text { Electricity das and } \\
\text { water }\end{array}$ & EGW & Electricity, Gas manufacture distribution, Water & Ely, gdt ,wtr \\
\hline Construction & CNS & Construction & cns \\
\hline Wholesale trade & TRD & Trade & $\operatorname{trd}$ \\
\hline Land transport & OTP & Transport nec & otp \\
\hline Water transport & WTP & Sea transport & wtp \\
\hline Air transport & ATP & Air transport & atp \\
\hline $\begin{array}{l}\text { Post and } \\
\text { communications }\end{array}$ & CMN & Communication & $\mathrm{cmn}$ \\
\hline Banking & OFI & Financial services nec & ofi \\
\hline Insurance & ISR & Insurance & isr \\
\hline Business activities & OBS & Business services nec & obs \\
\hline Other services & OSV & $\begin{array}{l}\text { Recreation and other services, } \\
\text { PubAdmin/Defence/Health/Educat } \\
\text { Dwellings }\end{array}$ & Ros, osg ,dwe \\
\hline
\end{tabular}

Source: GTAP 6. 
Tableau A5.2. Régions définies par référence aux régions GTAP-5

\begin{tabular}{|c|c|c|}
\hline Region & GTAP code & Country \\
\hline Australia & AUS & Australia \\
\hline New Zealand & NZL & New Zealand \\
\hline Canada & CAN & Canada \\
\hline \multicolumn{3}{|l|}{ China } \\
\hline \multicolumn{3}{|l|}{ Hong Kong-China } \\
\hline \multirow[t]{15}{*}{ EU15 } & AUT & Austria \\
\hline & BEL & Belgium \\
\hline & DNK & Denmark \\
\hline & FIN & Finland \\
\hline & FRA & France \\
\hline & DEU & Germany \\
\hline & GRC & Greece \\
\hline & IRL & Ireland \\
\hline & ITA & Italy \\
\hline & LUX & Luxembourg \\
\hline & NLD & Netherlands \\
\hline & PRT & Portugal \\
\hline & ESP & Spain \\
\hline & SWE & Sweden \\
\hline & GBR & United Kingdom \\
\hline \multirow[t]{11}{*}{ WEU } & HUN & Hungary \\
\hline & POL & Poland \\
\hline & $\mathrm{CHE}$ & Switzerland \\
\hline & TUR & Turkey \\
\hline & LTV & Latvia \\
\hline & LTU & Lithuania \\
\hline & EST & Estonia \\
\hline & CZE & Czech Republic \\
\hline & SVK & Slovak Republic \\
\hline & SLV & Slovenia \\
\hline & XEF & Rest of EFTA including Iceland, Lichtenstein, Norway \\
\hline JPN & JPN & Japan \\
\hline KOR & KOR & Korea \\
\hline MEX & MEX & Mexico \\
\hline USA & USA & United States of America \\
\hline Russia & RUS & Russia \\
\hline ROW & Rest of the World & All countries/regions not mentioned above \\
\hline List of countries from the ROW & ARG & Argentina \\
\hline \multirow{9}{*}{$\begin{array}{l}\text { group presented in the Charts } A 3.3 \\
\text { A3.3 to } A 3.7\end{array}$} & $\mathrm{BOL}$ & Bolivia \\
\hline & BRA & Brazil \\
\hline & MYS & Malaysia \\
\hline & SGP & Singapore \\
\hline & THA & Thailand \\
\hline & TWN & Chinese Taipei \\
\hline & $\mathrm{XCB}$ & $\begin{array}{l}\text { Rest of Caribbean including Anguilla, Aruba, Cayman } \\
\text { Islands, Cuba, Guadalupe, Martinique, British } \\
\text { Virgin Islands }\end{array}$ \\
\hline & XEA & $\begin{array}{l}\text { Rest of Asia including Macao, Mongolia and the } \\
\text { Democratic People's Republic of Korea }\end{array}$ \\
\hline & XME & $\begin{array}{l}\text { Rest of Middle East including Bahrain, Iran, Iraq, Israel, } \\
\text { Jordan, Kuwait, Lebanon, Oman, Qatar, Saudi } \\
\text { Arabia, Syrian Arab Republic, United Arab } \\
\text { Emirates, Yemen }\end{array}$ \\
\hline
\end{tabular}


TD/TC/WP(2006)10/FINAL

Tableau A5.3. Droits de douane moyens pondérés en fonction des échanges appliqués par la Chine, par partenaire commercial et par produit au cours de l'année de référence, 2001

\begin{tabular}{|c|c|c|c|c|c|c|c|c|c|c|c|c|c|c|}
\hline & Australia & $\begin{array}{c}\text { New } \\
\text { Zealand }\end{array}$ & China & $\begin{array}{l}\text { Hong } \\
\text { Kong- } \\
\text { China } \\
\end{array}$ & Russia & Japan & Korea & Canada & $\begin{array}{c}\text { United } \\
\text { States }\end{array}$ & Mexico & $\begin{array}{r}\text { EU } \\
15 \\
\end{array}$ & $\begin{array}{l}\text { Rest of } \\
\text { Western } \\
\text { Europe } \\
\end{array}$ & $\begin{array}{l}\text { Rest of } \\
\text { World }\end{array}$ & $\begin{array}{l}\text { Average for } \\
\text { product } \\
\text { category }\end{array}$ \\
\hline Agriculture and fishing & 19.3 & 12.9 & 0.0 & 27.1 & 23.9 & 9.0 & 14.5 & 20.7 & 68.4 & 4.5 & 21.0 & 11.9 & 65.1 & 50.4 \\
\hline Natural resources & 0.0 & 0.0 & 0.0 & 3.0 & 0.3 & 2.9 & 2.8 & 1.2 & 2.3 & 0.1 & 1.5 & 2.8 & 0.5 & 0.6 \\
\hline Coal & 4.5 & 4.5 & 0.0 & 0.0 & 4.3 & 3.5 & 0.0 & 0.0 & 4.5 & 0.0 & 3.6 & 0.0 & 4.1 & 4.3 \\
\hline Oil & 0.0 & 0.0 & 0.0 & 0.0 & 0.0 & 0.0 & 0.0 & 0.0 & 0.0 & 0.0 & 0.0 & 0.0 & 0.0 & 0.0 \\
\hline Food products and beverages & 17.1 & 20.3 & 0.0 & 33.5 & 16.7 & 24.3 & 22.2 & 19.5 & 18.5 & 9.6 & 24.5 & 17.4 & 15.4 & 18.3 \\
\hline Textiles, clothing and leather & 18.6 & 10.8 & 0.0 & 21.7 & 19.3 & 21.7 & 18.8 & 10.5 & 17.0 & 13.0 & 16.7 & 17.5 & 18.0 & 19.4 \\
\hline Chemicals and chemical products & 15.6 & 10.6 & 0.0 & 14.5 & 9.1 & 12.6 & 11.6 & 8.7 & 10.9 & 14.2 & 11.4 & 10.1 & 15.7 & 12.9 \\
\hline Other manufacturing & 14.7 & 8.0 & 0.0 & 14.1 & 3.3 & 14.6 & 16.0 & 2.3 & 10.4 & 18.5 & 14.5 & 14.3 & 9.9 & 11.7 \\
\hline Metal products & 11.4 & 7.2 & 0.0 & 4.7 & 5.5 & 8.0 & 9.4 & 4.2 & 5.3 & 7.1 & 9.0 & 4.2 & 6.4 & 7.5 \\
\hline Motor vehicles and parts & 22.5 & 14.9 & 0.0 & 17.8 & 17.6 & 42.3 & 47.7 & 32.5 & 30.3 & 23.9 & 36.2 & 43.9 & 35.4 & 38.1 \\
\hline Machinery and equipment & 13.4 & 12.4 & 0.0 & 13.8 & 6.0 & 13.0 & 12.9 & 8.0 & 10.3 & 14.5 & 12.1 & 12.8 & 13.5 & 12.1 \\
\hline Electronic equipment & 11.6 & 10.9 & 0.0 & 10.0 & 12.8 & 10.5 & 11.3 & 11.4 & 10.2 & 9.2 & 10.8 & 11.3 & 8.9 & 10.1 \\
\hline \multirow[t]{3}{*}{ Average for partner country } & 10.8 & 11.9 & 0.0 & 4.7 & 6.3 & 13.6 & 13.4 & 10.0 & 13.6 & 7.6 & 10.9 & 8.4 & 12.2 & \\
\hline & \multicolumn{13}{|c|}{ Après la mise en œuvre des engagements pris lors de l'accession à l'OMC } & \\
\hline & Australia & $\begin{array}{c}\text { New } \\
\text { Zealand } \\
\end{array}$ & China & $\begin{array}{l}\text { Hong } \\
\text { Kong- } \\
\text { China } \\
\end{array}$ & Russia & Japan & Korea & Canada & $\begin{array}{c}\text { United } \\
\text { States } \\
\end{array}$ & Mexico & $\begin{array}{l}\text { EU } \\
15 \\
\end{array}$ & $\begin{array}{c}\text { Rest of } \\
\text { Western } \\
\text { Europe } \\
\end{array}$ & $\begin{array}{c}\text { Rest of } \\
\text { World }\end{array}$ & $\begin{array}{c}\text { Average for } \\
\text { product } \\
\text { category } \\
\end{array}$ \\
\hline Agriculture and fishing & 18.9 & 8.3 & 0.0 & 10.0 & 15.3 & 8.1 & 10.8 & 19.6 & 4.7 & 6.3 & 20.2 & 9.3 & 6.3 & 9.7 \\
\hline Natural resources & 0.0 & 0.0 & 0.0 & 3.0 & 0.2 & 2.9 & 2.8 & 1.2 & 2.2 & 0.1 & 1.5 & 2.7 & 0.6 & 0.5 \\
\hline Coal & 4.5 & 4.5 & 0.0 & 3.5 & 4.3 & 3.5 & 4.1 & 5.0 & 4.5 & 3.7 & 4.8 & 4.4 & 4.2 & 4.4 \\
\hline Oil & 0.0 & 0.0 & 0.0 & 0.0 & 0.0 & 3.0 & 0.0 & 6.0 & 0.2 & 3.0 & 0.1 & 0.0 & 0.0 & 0.0 \\
\hline Food products and beverages & 11.5 & 10.0 & 0.0 & 17.5 & 9.8 & 13.8 & 14.8 & 9.6 & 11.2 & 5.3 & 12.0 & 11.1 & 10.9 & 11.2 \\
\hline Textiles, clothing and leather & 16.0 & 10.1 & 0.0 & 13.7 & 12.0 & 9.2 & 9.4 & 5.3 & 8.9 & 6.8 & 9.5 & 9.4 & 8.6 & 9.6 \\
\hline Chemicals and chemical products & 8.6 & 6.9 & 0.0 & 7.2 & 6.0 & 7.2 & 6.4 & 5.1 & 6.6 & 7.7 & 7.1 & 6.7 & 11.4 & 8.2 \\
\hline Other manufacturing & 7.2 & 3.5 & 0.0 & 7.5 & 1.4 & 10.9 & 9.8 & 0.8 & 6.2 & 9.9 & 8.6 & 8.7 & 5.9 & 7.2 \\
\hline Metal products & 6.0 & 3.8 & 0.0 & 3.7 & 4.4 & 5.4 & 6.0 & 3.1 & 3.6 & 4.3 & 5.9 & 3.6 & 4.9 & 5.1 \\
\hline Motor vehicles and parts & 12.2 & 10.3 & 0.0 & 9.2 & 11.9 & 15.9 & 18.3 & 11.4 & 13.8 & 8.9 & 14.6 & 16.3 & 13.9 & 15.0 \\
\hline Machinery and equipment & 6.9 & 6.4 & 0.0 & 7.5 & 5.0 & 6.7 & 6.7 & 4.9 & 5.2 & 7.8 & 6.9 & 7.7 & 7.4 & 6.6 \\
\hline Electronic equipment & 1.1 & 0.8 & 0.0 & 2.0 & 4.0 & 2.4 & 4.2 & 1.0 & 0.6 & 0.9 & 1.4 & 1.9 & 1.2 & 1.8 \\
\hline Average for partner country & 8.0 & 6.5 & 0.0 & 2.6 & 4.4 & 6.2 & 6.8 & 6.1 & 4.0 & 2.0 & 5.3 & 4.8 & 5.1 & \\
\hline
\end{tabular}


TD/TC/WP(2006)10/FINAL

Diagramme A5.1. Droits de douane moyens pondérés en fonction des échanges appliqués par la Chine, par partenaire commercial (année de référence : 2001)

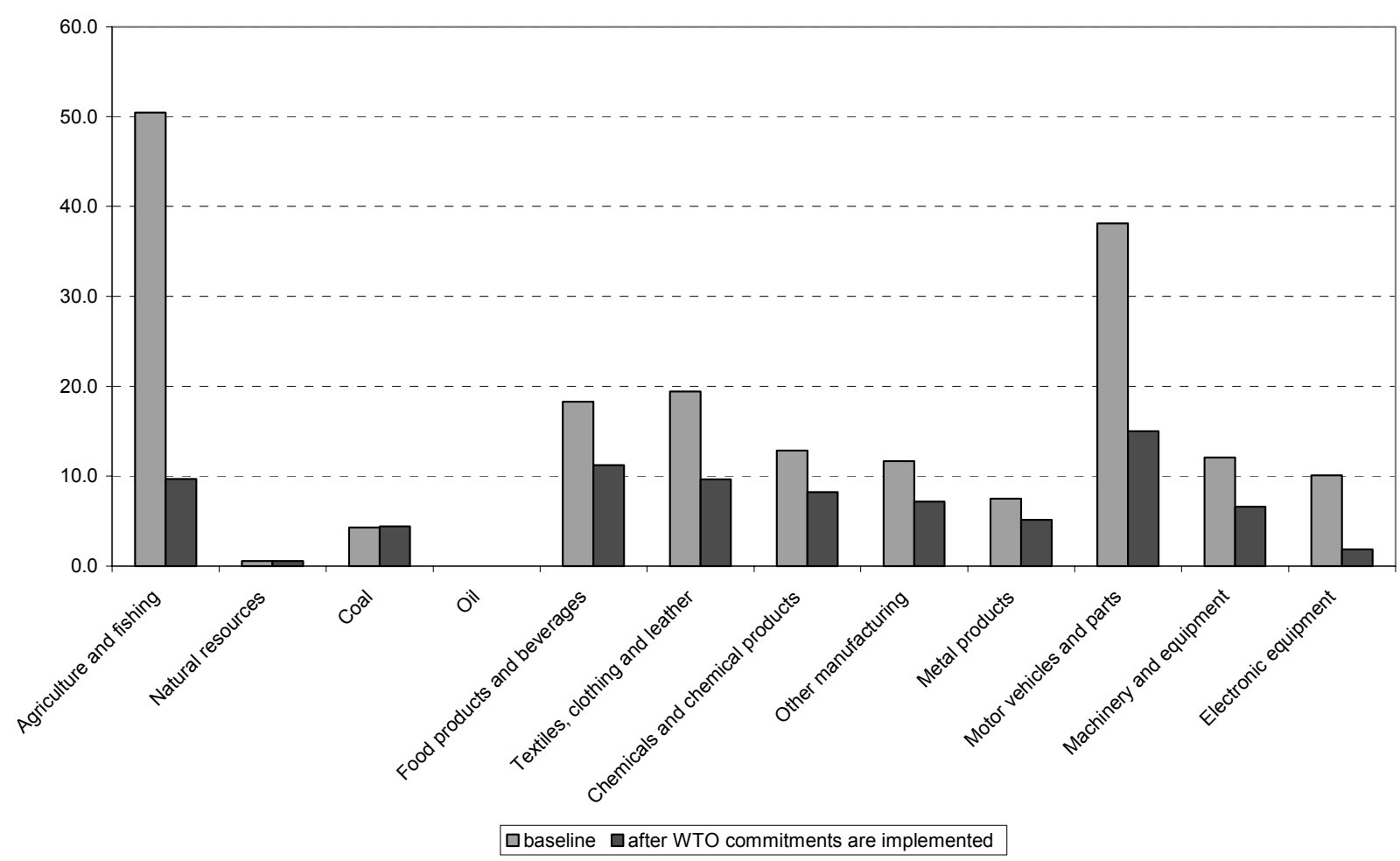

Source : Calculs des auteurs à partir de la base de données GTAP 6. 
TD/TC/WP(2006)10/FINAL

Diagramme A5.2. Droits de douane moyens pondérés en fonction des échanges appliqués par la Chine, par catégorie de produits (année de référence : 2001)

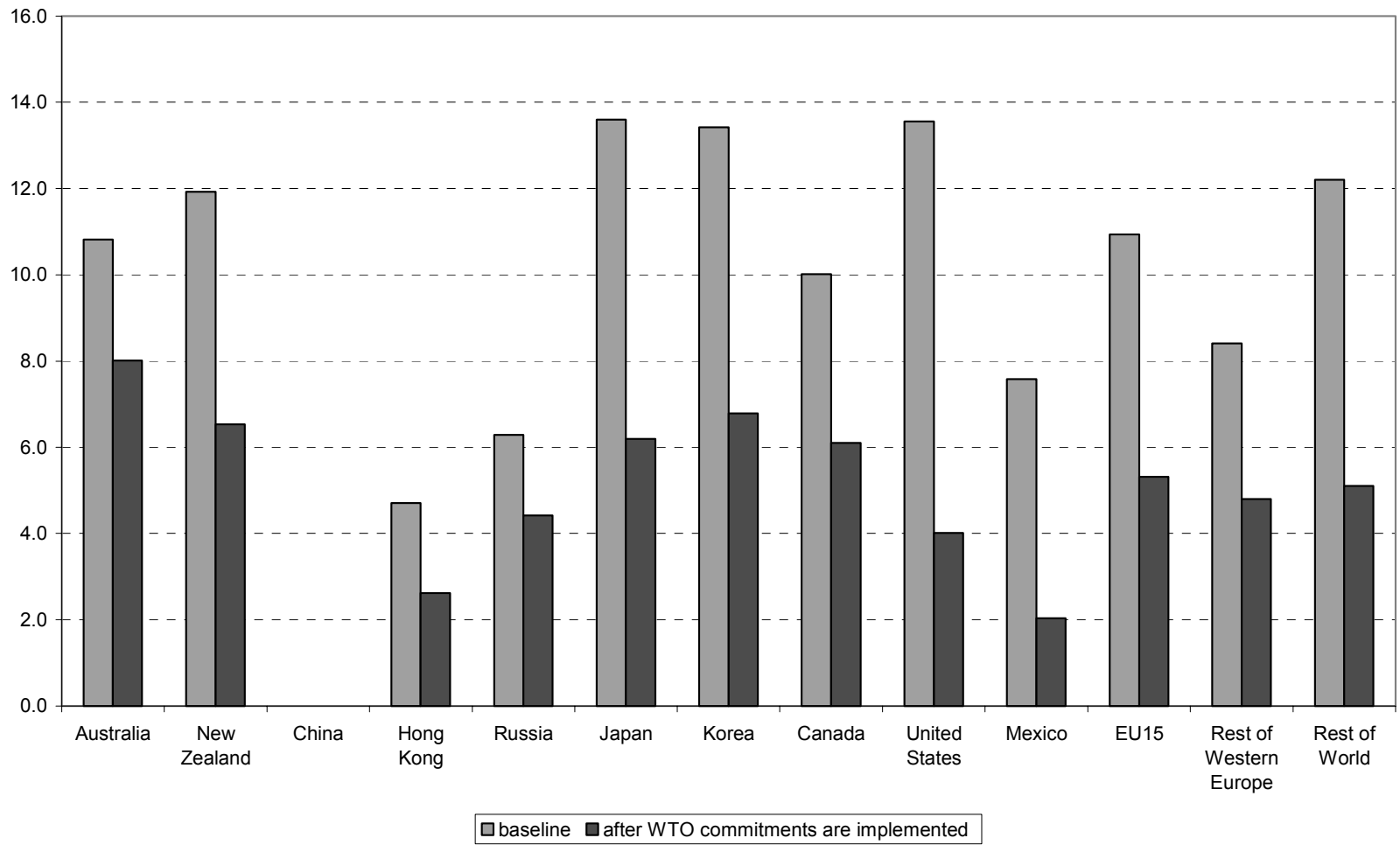

Source : Calculs des auteurs à partir de la base de données GTAP 6. 
TD/TC/WP(2006)10/FINAL

Tableau A5.4. Équivalents de taxes dans le secteur des services en Chine (avant et après la mise en œuvre des engagements pris dans le cadre de l'AGCS)

\begin{tabular}{lll}
\hline & China & $\begin{array}{l}\text { China } \\
\text { WTO }\end{array}$ \\
\hline Banking mode 1 & 2.0 & 0.06 \\
Banking mode 3 & 21.5 & 8.17 \\
Insurance mode 1 & 130.3 & 67.89 \\
Insurance mode 3 & 88.2 & 34.80 \\
Telecommunication mode 1 & 15.7 & 7.06 \\
Telecommunication mode 3 & 38.3 & 25.95 \\
Trade mode 1 & 13.7 & 6.61 \\
Trade mode 3 & 7.2 & 2.06 \\
Professional services mode 1 & 107.3 & 43.91 \\
Professional services mode 3 & 10.3 & 4.78 \\
\hline
\end{tabular}

Source : Calculs des auteurs sur la base de la méthode décrite in OCDE (2005j) [TD/TC/WP(2005)36]. 
TD/TC/WP(2006)10/FINAL

Tableau A5.5. Décomposition des effets sur le bien-être de la mise en œuvre par la Chine des engagements pris à l'OMC dans le domaine des marchandises (millions USD)

\begin{tabular}{|c|c|c|c|c|c|c|c|c|c|c|c|c|c|c|}
\hline & Australia & $\begin{array}{c}\text { New } \\
\text { Zealand }\end{array}$ & China & $\begin{array}{l}\text { Hong Kong- } \\
\text { China }\end{array}$ & Russia & Japan & Korea & Canada & $\begin{array}{l}\text { United } \\
\text { States }\end{array}$ & Mexico & EU15 & $\begin{array}{l}\text { Rest of } \\
\text { Western } \\
\text { Europe }\end{array}$ & $\begin{array}{l}\text { Rest of } \\
\text { World }\end{array}$ & Total \\
\hline Total welfare change & -227 & 14 & 13,761 & 1,072 & -142 & 2,451 & 647 & -232 & 4,272 & -164 & 3,899 & -36 & $-1,149$ & 24,167 \\
\hline \multicolumn{15}{|c|}{ Main components of welfare change } \\
\hline Allocative efficiency & -1 & -1 & 20,804 & -39 & -25 & 224 & 102 & 91 & 1,090 & -67 & 1,978 & -34 & -556 & 23,566 \\
\hline Net capital endowment & -3 & -2 & 839 & 31 & -1 & 41 & 17 & -7 & -114 & -4 & -40 & -34 & -284 & 438 \\
\hline Terms of trade & -116 & -5 & $-7,098$ & 309 & -53 & 1,179 & 439 & 48 & 3,646 & -240 & 2,425 & -157 & -377 & 0 \\
\hline Foreign debt effects & -141 & -5 & -76 & 77 & -94 & 1,037 & -1 & -422 & 200 & -290 & 266 & 184 & -735 & 0 \\
\hline FDI effects & 8 & 21 & $-1,559$ & 696 & 26 & -16 & 78 & 35 & -224 & 378 & -593 & 48 & 1,102 & 0 \\
\hline FDI-related rents & 2 & 0 & 19 & -9 & 1 & -9 & -4 & 13 & -63 & 59 & -32 & -4 & 26 & 0 \\
\hline Product variety & 26 & 6 & 832 & 7 & 4 & -1 & 19 & 10 & -277 & -5 & -182 & -47 & -325 & 68 \\
\hline Other & -2 & 0 & 0 & 0 & 0 & -4 & -2 & -1 & 14 & 6 & 78 & 7 & 0 & 95 \\
\hline
\end{tabular}

Tableau A5.6. Décomposition des effets sur le bien-être d'une totale libéralisation par la Chine des échanges dans le domaine des marchandises (millions USD)

\begin{tabular}{|c|c|c|c|c|c|c|c|c|c|c|c|c|c|c|}
\hline & Australia & $\begin{array}{c}\text { New } \\
\text { Zealand }\end{array}$ & China & $\begin{array}{l}\text { Hong Kong- } \\
\text { China }\end{array}$ & Russia & Japan & Korea & Canada & $\begin{array}{l}\text { United } \\
\text { States }\end{array}$ & Mexico & $\begin{array}{l}\mathrm{EU} \\
15 \\
\end{array}$ & $\begin{array}{c}\text { Rest of } \\
\text { Western } \\
\text { Europe }\end{array}$ & $\begin{array}{l}\text { Rest of } \\
\text { World }\end{array}$ & Total \\
\hline Total welfare change & -45 & 69 & 15,952 & 2,009 & 479 & 6,280 & 2,179 & -78 & 4,614 & -30 & -116 & 522 & 2,700 & 34,535 \\
\hline \multicolumn{15}{|c|}{ Main components of welfare change } \\
\hline Allocative efficiency & 41 & 12 & 29,086 & -43 & 51 & 835 & 740 & 111 & 1,219 & -103 & $\begin{array}{c}1,88 \\
4\end{array}$ & 35 & 363 & 34,229 \\
\hline Net capital endowment & -5 & -3 & 958 & 15 & 0 & 84 & 37 & -8 & -332 & -10 & $\begin{array}{c}-66 \\
2,69\end{array}$ & -43 & -384 & 244 \\
\hline Terms of trade & 132 & 24 & $-12,711$ & 934 & 77 & 2,474 & 1,052 & 148 & 4,533 & -278 & 8 & -5 & 923 & 0 \\
\hline Foreign debt effects & -241 & 38 & -377 & 267 & 378 & 3,082 & 388 & -448 & -452 & -327 & תרי & 574 & 889 & 0 \\
\hline FDI effects & 14 & -2 & $-2,480$ & 1,139 & -14 & -23 & 33 & 83 & 222 & 570 & -720 & 9 & 1,169 & 0 \\
\hline FDI-related rents & -3 & -4 & 711 & -323 & -11 & -104 & -77 & 40 & -235 & 121 & -201 & 8 & 79 & 0 \\
\hline Product variety & 17 & 4 & 704 & 21 & -1 & -65 & 10 & -4 & -358 & -7 & -109 & -69 & -332 & -191 \\
\hline Other & 0 & 0 & 61 & 1 & -1 & -3 & -4 & 1 & 16 & 5 & 168 & 14 & -6 & 253 \\
\hline
\end{tabular}


TD/TC/WP(2006)10/FINAL

Tableau A5.7. Décomposition des effets sur le bien-être de la mise en œuvre par la Chine des engagements pris à l'OMC dans le domaine des

services (millions USD)

\begin{tabular}{|c|c|c|c|c|c|c|c|c|c|c|c|c|c|c|}
\hline & Australia & New Zealand & China & $\begin{array}{l}\text { Hong Kong- } \\
\text { China }\end{array}$ & Russia & Japan & Korea & Canada & United States & Mexico & EU15 & $\begin{array}{l}\text { Rest of } \\
\text { Europe }\end{array}$ & $\begin{array}{l}\text { Rest of } \\
\text { World }\end{array}$ & Total \\
\hline Total welfare change & 20.47 & -2.37 & 2820.99 & 278.19 & -26.51 & 62.90 & 26.25 & 2.74 & 614.97 & 19.38 & 388.65 & -1.28 & 281.69 & 4486.06 \\
\hline \multicolumn{15}{|c|}{ Main components of welfare change } \\
\hline Allocative efficiency & 8.70 & 0.49 & 3903.15 & -4.96 & -3.40 & -7.37 & 12.73 & 13.77 & 73.78 & 6.59 & 104.16 & -4.04 & 36.62 & 4140.23 \\
\hline Net capital endowment & -1.30 & 0.16 & 139.87 & 15.69 & -0.12 & 5.29 & 2.27 & -0.98 & -26.93 & -6.33 & 20.49 & -0.27 & -5.17 & 142.68 \\
\hline Terms of trade & 16.82 & 1.86 & -1595.20 & 449.84 & -28.85 & 80.85 & 6.90 & -6.12 & 478.91 & -16.12 & 446.83 & 48.39 & 115.90 & 0.00 \\
\hline Foreign debt effects & 4.52 & 0.28 & -137.79 & 5.84 & 5.34 & -12.19 & 0.33 & 12.68 & 31.40 & 8.77 & 70.47 & -6.03 & 16.38 & 0.00 \\
\hline FDI effects & 4.21 & -3.44 & -1103.80 & 229.22 & 2.07 & 100.02 & 55.07 & 8.20 & 236.64 & 30.94 & 23.60 & -10.20 & 427.49 & 0.00 \\
\hline FDI-related rents & -6.90 & -1.03 & 934.20 & -426.90 & -0.11 & -80.82 & -38.33 & -10.22 & -79.48 & -0.21 & -85.92 & -5.43 & -198.85 & 0.00 \\
\hline Product variety & -5.57 & -0.68 & 550.47 & 9.40 & -1.47 & -23.08 & -12.75 & -14.61 & -99.28 & -4.24 & -184.53 & -23.01 & -109.54 & 81.11 \\
\hline
\end{tabular}

Source : projections du modèle FTAP

Tableau A5.8. Décomposition des effets sur le bien-être d'une totale libéralisation par la Chine des échanges dans le domaine des services (millions USD)

\begin{tabular}{|c|c|c|c|c|c|c|c|c|c|c|c|c|c|c|}
\hline & Australia & New Zealand & China & $\begin{array}{l}\text { Hong Kong- } \\
\text { China } \\
\end{array}$ & Russia & Japan & Korea & Canada & United States & Mexico & EU15 & $\begin{array}{l}\text { Rest of } \\
\text { Europe } \\
\end{array}$ & $\begin{array}{l}\text { Rest of } \\
\text { World } \\
\end{array}$ & Total \\
\hline Total welfare change & -99 & -3 & 11,973 & 719 & -191 & 1,089 & -63 & -377 & 575 & -93 & -96 & 66 & 230 & 13,730 \\
\hline \multicolumn{15}{|c|}{ Main components of welfare change } \\
\hline Allocative efficiency & -3 & -1 & 13,186 & -17 & -29 & -54 & -30 & -20 & -3 & -15 & 22 & -20 & -94 & 12,922 \\
\hline Net capital endowment & -5 & -1 & 477 & 19 & -3 & -8 & 3 & -12 & -239 & -14 & -26 & -16 & -176 & -1 \\
\hline Terms of trade & 35 & 6 & $-2,181$ & 992 & -81 & -4 & -47 & -26 & 610 & -49 & 503 & 36 & 205 & 0 \\
\hline Foreign debt effects & -129 & -6 & -249 & 80 & -85 & 1,225 & -13 & -385 & 58 & -228 & 256 & 169 & -694 & 0 \\
\hline FDI effects & 24 & 1 & $-2,984$ & 620 & 14 & 212 & 132 & 90 & 503 & 188 & -350 & -57 & 1,607 & 0 \\
\hline FDI-related rents & -13 & -1 & 2,193 & $-1,006$ & 2 & -194 & -89 & -4 & -232 & 28 & -247 & -13 & -423 & 0 \\
\hline Product variety & -7 & -1 & 1,275 & 30 & -10 & -91 & -21 & -20 & -124 & -3 & -289 & -36 & -194 & 509 \\
\hline
\end{tabular}

Source : projections du modèle FTAP. 
TD/TC/WP(2006)10/FINAL

Tableau A5.9. Mise en œuvre des engagements pris par la Chine à l'OMC dans le domaine des marchandises : pourcentage de variation des stocks d'IDE détenus en Chine, par région effectuant l'investissement et par secteur

\begin{tabular}{|c|c|c|c|c|c|c|c|c|c|c|c|c|c|c|}
\hline & Australia & $\begin{array}{c}\text { New } \\
\text { Zealand } \\
\end{array}$ & China & $\begin{array}{l}\text { Hong } \\
\text { Kong- } \\
\text { China } \\
\end{array}$ & Russia & Japan & Korea & Canada & $\begin{array}{l}\text { United } \\
\text { States } \\
\end{array}$ & Mexico & EU15 & $\begin{array}{c}\text { Rest of } \\
\text { Western } \\
\text { Europe } \\
\end{array}$ & $\begin{array}{l}\text { Rest of } \\
\text { World }\end{array}$ & $\begin{array}{c}\text { All } \\
\text { countries } \\
\end{array}$ \\
\hline Agriculture and fishing & -1.27 & -1.34 & 0.27 & -1.27 & -1.32 & -1.31 & -1.32 & -1.31 & -1.34 & -1.3 & -1.32 & -1.33 & -1.32 & 0.4 \\
\hline Natural resources & -0.42 & -0.46 & 0.22 & -0.39 & -0.44 & -0.42 & -0.45 & -0.43 & -0.44 & -0.42 & -0.44 & -0.45 & -0.44 & 0.21 \\
\hline Coal & -0.07 & -0.1 & 0.19 & -0.03 & -0.08 & -0.07 & -0.09 & -0.08 & -0.08 & -0.06 & -0.08 & -0.09 & -0.08 & 0.15 \\
\hline Oil & -0.21 & -0.25 & 0.2 & -0.17 & -0.23 & -0.21 & -0.23 & -0.22 & -0.22 & -0.2 & -0.22 & -0.23 & -0.23 & 0.15 \\
\hline Food products and beverages & 0.69 & 0.63 & 0.13 & 0.74 & 0.68 & 0.7 & 0.66 & 0.69 & 0.69 & 0.72 & 0.69 & 0.68 & 0.67 & 0.11 \\
\hline $\begin{array}{l}\text { Textiles, clothing and leather } \\
\text { Chemicals and chemical }\end{array}$ & 2.8 & 2.76 & 0.03 & 2.71 & 2.8 & 2.75 & 2.69 & 2.83 & 2.8 & 2.83 & 2.82 & 2.82 & 2.72 & -0.51 \\
\hline products & -0.22 & -0.27 & 0.19 & -0.16 & -0.25 & -0.23 & -0.27 & -0.23 & -0.24 & -0.21 & -0.24 & -0.25 & -0.25 & 0.24 \\
\hline Other manufacturing & 0.11 & 0.06 & 0.17 & 0.16 & 0.09 & 0.11 & 0.07 & 0.1 & 0.09 & 0.12 & 0.09 & 0.08 & 0.08 & 0.22 \\
\hline Metal products & -0.13 & -0.17 & 0.19 & -0.05 & -0.15 & -0.12 & -0.15 & -0.13 & -0.14 & -0.11 & -0.14 & -0.15 & -0.14 & 0.2 \\
\hline Motor vehicles and parts & -2.23 & -2.27 & 0.29 & -2.02 & -2.26 & -2.23 & -2.19 & -2.25 & -2.25 & -2.23 & -2.25 & -2.27 & -2.2 & 0.29 \\
\hline Machinery and equipment & -0.21 & -0.26 & 0.19 & -0.14 & -0.24 & -0.22 & -0.24 & -0.23 & -0.24 & -0.2 & -0.23 & -0.25 & -0.24 & 0.3 \\
\hline Electronic equipment & 1.18 & 1.13 & 0.2 & 1.14 & 1.17 & 1.18 & 1.11 & 1.18 & 1.17 & 1.2 & 1.17 & 1.16 & 1.11 & 0.23 \\
\hline Electricity & 0.14 & 0.08 & 0.17 & 0.19 & 0.12 & 0.13 & 0.09 & 0.13 & 0.12 & 0.15 & 0.12 & 0.11 & 0.11 & 0.17 \\
\hline Wholesale trade & 0.47 & 0.42 & 0.16 & 0.51 & 0.44 & 0.46 & 0.43 & 0.46 & 0.45 & 0.48 & 0.45 & 0.44 & 0.44 & 0.17 \\
\hline Water transport & 0.41 & 0.36 & 0.15 & 0.45 & 0.38 & 0.4 & 0.36 & 0.39 & 0.38 & 0.42 & 0.38 & 0.37 & 0.38 & 0.25 \\
\hline Air transport & 0.46 & 0.41 & 0.17 & 0.51 & 0.43 & 0.45 & 0.42 & 0.45 & 0.44 & 0.47 & 0.44 & 0.43 & 0.43 & 0.16 \\
\hline Post and communications & 0.41 & 0.36 & 0.16 & 0.46 & 0.38 & 0.4 & 0.37 & 0.4 & 0.39 & 0.42 & 0.39 & 0.38 & 0.38 & 0.16 \\
\hline Finance & 0.41 & 0.36 & 0.16 & 0.47 & 0.39 & 0.4 & 0.37 & 0.4 & 0.39 & 0.42 & 0.39 & 0.38 & 0.38 & 0.14 \\
\hline Insurance & 0.35 & 0.29 & 0.17 & 0.4 & 0.32 & 0.34 & 0.31 & 0.33 & 0.32 & 0.35 & 0.33 & 0.31 & 0.32 & 0.13 \\
\hline Business activities & 0.34 & 0.29 & 0.17 & 0.36 & 0.31 & 0.33 & 0.29 & 0.32 & 0.32 & 0.35 & 0.32 & 0.3 & 0.31 & 0.16 \\
\hline Other services & 0.5 & 0.45 & 0.15 & 0.54 & 0.48 & 0.49 & 0.46 & 0.49 & 0.48 & 0.51 & 0.48 & 0.47 & 0.47 & 0.17 \\
\hline
\end{tabular}

Source : projections du modèle FTAP. 
TD/TC/WP(2006)10/FINAL

Tableau A5.10. Accession à l'OMC de la Chine dans le domaine des services : pourcentage de variation des stocks d'IDE détenus en Chine, par région d'origine de l'investissement et par secteur

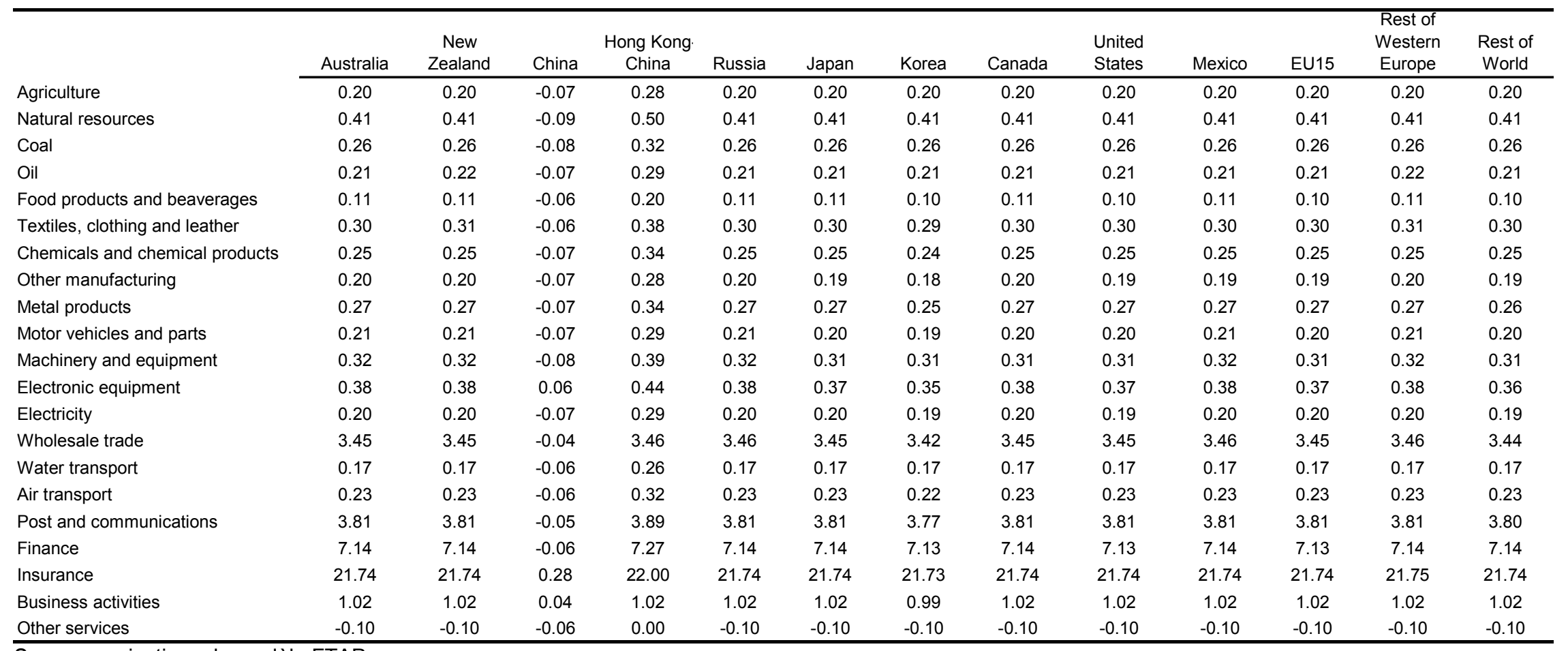

Source : projections du modèle FTAP. 